\title{
STALL MITIGATION USING WING SPOILERS
}

By

\section{Harpuneet Kaur Pabla, B.Eng}

Aerospace Engineering

Ryerson University, 2012

\begin{abstract}
A thesis presented to Ryerson University
in partial fulfillment of the requirements for the degree of Master of Applied Science

In the Program of Aerospace Engineering
\end{abstract}

Toronto, Ontario, Canada, 2014

(C) Harpuneet Pabla 


\section{AUTHOR'S DECLARATION}

I hereby declare that I am the sole author of this thesis. This is a true copy of the thesis, including any required final revisions, as accepted by my examiners.

I authorize Ryerson University to lend this thesis to other institutions or individuals for the purpose of scholarly research

I further authorize Ryerson University to reproduce this thesis by photocopying or by other means, in total or in part, at the request of other institutions or individuals for the purpose of scholarly research.

I understand that my thesis may be made electronically available to the public. 
Stall Mitigation Using Wing Spoilers

\section{Harpuneet Pabla, Master of Applied Science, Aerospace Engineering \\ Ryerson University, Toronto 2014}

The objective of this thesis was to conduct a two dimensional Compuational Fluid Dyanmic analysis on wing-spoiler lift effectiveness in delaying stall effects using the NACA 2412 airfoil section. The project was divided into three sub-areas; grid-independent study, the baseline study and spoiler performance study. The grid independent study was carried out for the purpose of mesh optimization, i.e. to determine the point at which computed solutions had little or no change in value with increasing number of mesh nodes. This study was conducted at an angle of attack of 16 degrees as it served as both a high pitch angle value as well as a pre-stall point in which unsteady effects were not a determining influence that may have served as a deterrent in resolving potential grid error. The baseline study was conducted to establish a data foundation to be used as a comparison to the spoiler study to effectively determine its effects on the lift performance. Results for the baseline study were shown to match experimental values most closely using the Transition SST Turbulence Model at a Mach number of 0.17. Therefore to remain consistent the spoiler study was carried out for the same Mach value with the viscosity of $1.84 \mathrm{E}-5[\mathrm{~kg} / \mathrm{ms}]$ and Temperature of $300[\mathrm{~K}]$, which produced a Reynolds number of approximately 3.79E6. Therefore, using the same flight conditions employed in the baseline study, the spoiler grids were generated in ANSYS ICEM CFD and imported into Fluent and solved using the Transition SST turbulence model. Results for spoiler deflections of 4, and 10 degrees were carried out across spoiler locations of 60\%, 65 and 70\% leading edge chord-wise locations. Results were shown to be optimal for 4 degree spoiler deflections across all locations tested, with highest values for lift effectiveness recorded at $70 \%$ leading edge. The findings of this thesis provides much potential for utilizing spoilers as lift enhancing devices and adds an alternate perspective in improving aircraft performance. 


\section{ACKNOWLEDGEMENTS}

I would sincerely like to extend my gratitude to my Supervisor, Professor Paul Walsh, for his tremendous dedication, patience, and expertise during the course of my thesis. Under your direction, I have learned to further my abilities and gained a renewed self-confidence; to continue to move forward and exceed, particularly during times of stress, when things were not proceeding to plan.

I would also to thank Industry Leads, Mr. Neal A. Harrison, and Mr. Alex Tsoulis, for their consistent support and much appreciated guidance. Anytime I required assistance, they were always willing and ready to be available; no questions asked; without any hesitation. I am deeply grateful for their time and do Thank them sincerely.

Last but definitely not the least, I would like to thank my amazing family for all their love and support; for any success I have today is undoubtedly because of their immense support and words of encouragement. Especially, to my Parents I am forever thankful to them. You have always put my needs first before yours and honestly no words can explain how grateful I am for all your sacrifices. I feel truly honoured and privileged to call you my Parents.

It has been a wonderful journey and excellent end to my academic career; I look forward to applying all I have learned as I begin the next phase in my life.

Harpuneet Pabla

Ryerson University

September 2014 


\section{Table of Contents}

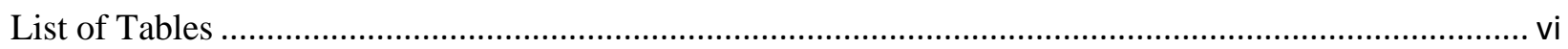

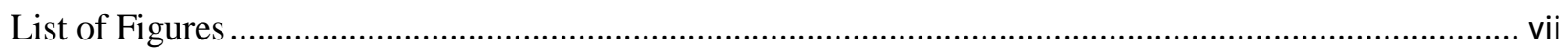

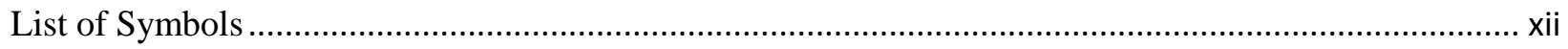

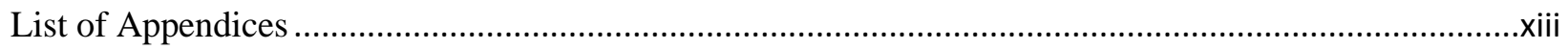

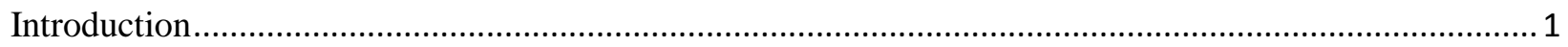

1.0 Motivation

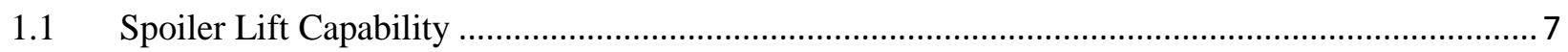

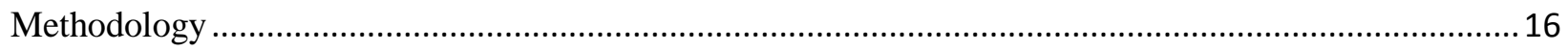

2.0 Baseline Mesh Information........................................................................................................

2.0.1 Baseline Blocking \& Nodal Mesh Details ..............................................................................19

2.1 Spoiler-airfoil Geometry and Mesh Details ..............................................................................19

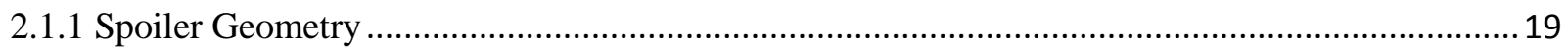

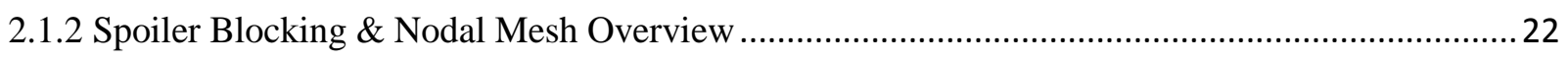

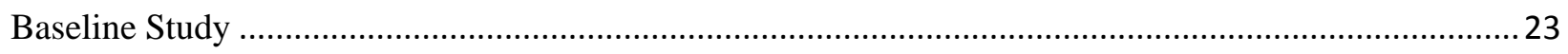

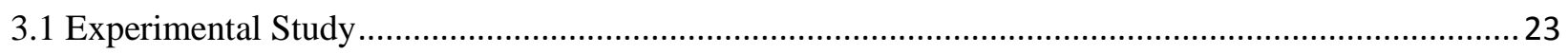

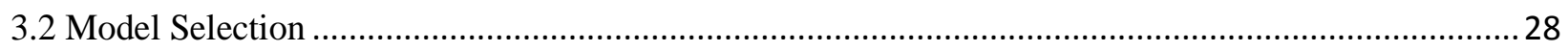

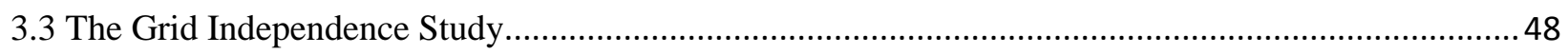

3.3.1 Grid Independent Study Calculations …………………………………………………......

3.3.1.1 Grid Convergence Study using three Grids for Analysis ......................................................5

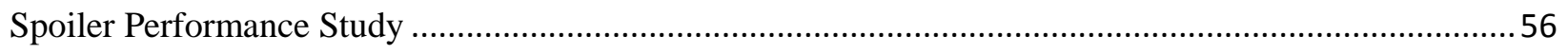

4.1 Spoiler Performance Results ................................................................................................. 56

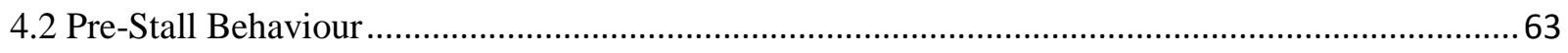

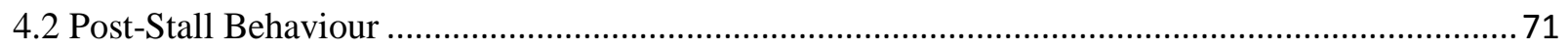

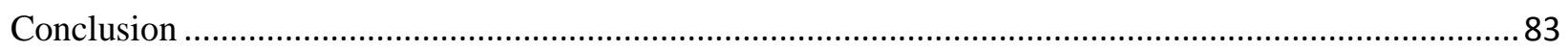

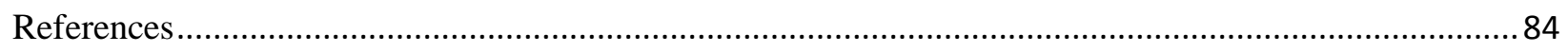




\section{List of Tables}

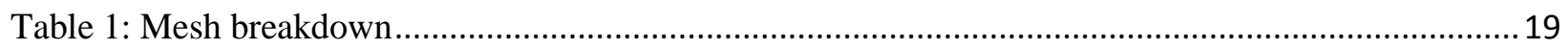

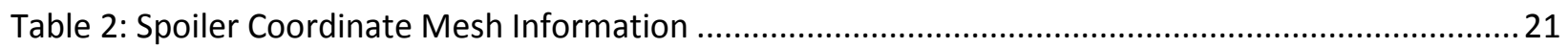

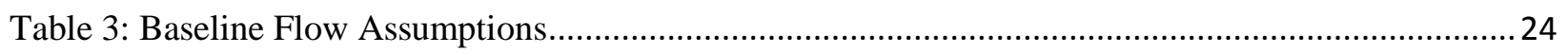

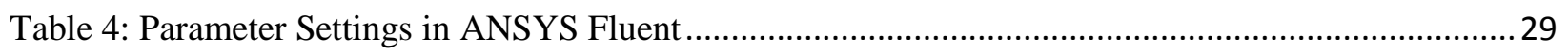

Table 5: Drag Coefficient Values for Clean NACA2412 Mesh, M=0.17, Re=3.79E6 ...........................34

Table 6: Grid Independent Study Results for Baseline Mesh, Alpha=16 ............................................51

Table 7: Spoiler Study Results for alpha of 19 degrees, Transition SST model......................................62

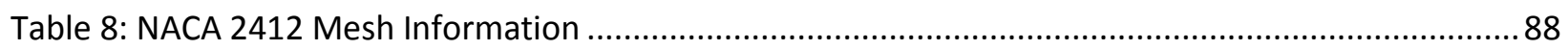

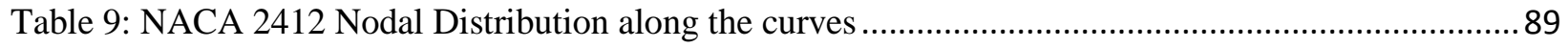

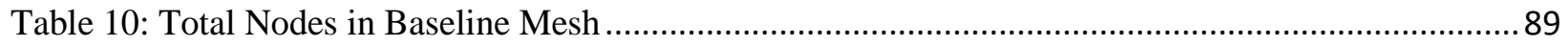

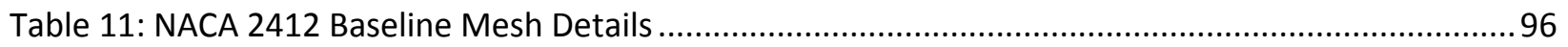

Table 12: NACA2412 Airfoil-Spoiler Mesh Details Cont'd .................................................................98

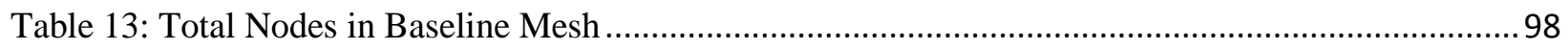




\section{List of Figures}

Figure 1: Pressure Distribution on the Upper surface across various spoiler deflections [1] ................... 2

Figure 2: Lift-alpha plot for various spoiler deflections [1] ............................................................ 3

Figure 3: Lift-alpha curve for NACA4424-spoiler section [2] .......................................................... 4

Figure 4: Lift-alpha curve for Boeing airfoil-spoiler (73.3\%LE) [4] ...................................................... 5

Figure 5: Lift-alpha plot of LS(1)-0413 airfoil-spoiler (77.5\%LE) [4] ................................................ 6

Figure 6: Variation in $\mathrm{Cp}$ with changing Spoiler deflection [5] ..................................................... 7

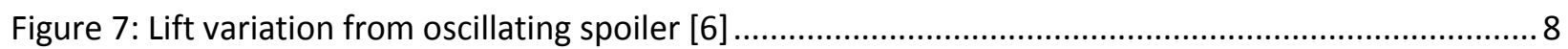

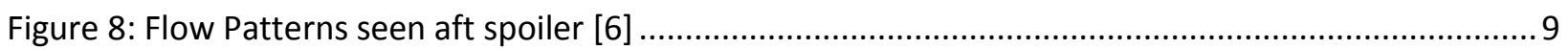

Figure 9: Pressure and Streamline Distribution for various spoiler deflections [8]...............................10

Figure 10: Lift Effectiveness vs. Chord Location with Mach [9] ...........................................................11

Figure 11: Cl-alpha curve for fixed spoiler deflection [11] ................................................................12

Figure 12: Pressure Distribution for Effector fixed at 30 degrees [11] ................................................13

Figure 13: Lift Coefficient, S824, Reynolds Number= 1 million [12] ................................................... 14

Figure 14: Pressure Distribution S824, 0.86\% chord location, Alpha = 16, Re= 1 million [12] .................15

Figure 15: Airfoil parameter convention [13] .................................................................................. 17

Figure 16: Streamlines for pre-stall, near-stall and post stall $\alpha$ values for NACA2412 at Reynolds Number

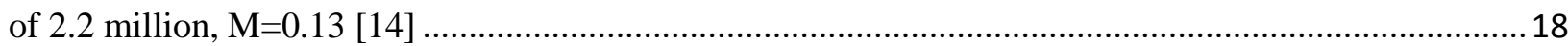

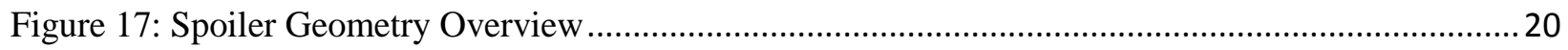

Figure 18: NACA 2412 airfoil-Spoiler deflection overview .............................................................22

Figure 19: Baseline Study, Lift Coefficient, Transition SST Model ....................................................26

Figure 20: Baseline Study, Drag Coefficient, Transition SST Model .......................................................26

Figure 21: Baseline Study, Moment Coefficient at Quatre Chord, Transition SST Model .......................27

Figure 22: Mean velocity distribution U+ vs. Wall Y+ [18] ............................................................. 31

Figure 23: Lift-alpha Plot for Model Study of NACA2412 airfoil Clean Mesh, M=0.17, Re=3.79E6..........32

Figure 24: Drag- alpha plot for Model Study of NACA2412 airfoil Clean Mesh, $M=0.17, \operatorname{Re}=3.79 E 6$........33

Figure 25: Model Study for alpha=16, M=0.17, Re=3.79E6, sstkw, k-epsilon, S-A and Transition SST....... 35

Figure 26: Model Study, Wall shear Stress Plots for Alpha=16, Clean Mesh, M=0.17, Re=3.798E6 .......36

Figure 27: Truncated Wall Shear Stress Plot, Baseline Mesh, Alpha=16 (M=0.17, Re=3.79E6) .............36

Figure 28: Wall y+ Plot for Alpha=16, Baseline Mesh ( $M=0.17, \operatorname{Re}=3.79 E 6)$.........................................37

Figure 29: Pressure Coefficient Plot for Clean Mesh, Alpha=16 (Mach=0.17, Re=3.79E6) ....................37 
Figure 30: Velocity Contours for Baseline Mesh, Alpha=17, sstkw Model ( $M=0.17, \operatorname{Re}=3.79 E 6)$ .40

Figure 31: Velocity Contours for Baseline Mesh, Alpha=17, S-A Model ( $M=0.17, R e=3.79 E 6)$................40

Figure 32: Velocity Contours for Baseline Mesh, Alpha=17, Transition SST Model (M=0.17, Re=3.79E6).40

Figure 33: Wall Shear Stress, Baseline Mesh, Alpha=17 (M=0.17, Re=3.79E6)..................................41

Figure 34: Truncated Wall Shear Stress Plot, Baseline Mesh, Alpha=17 (M=0.17, Re=3.79E6) ............41

Figure 35: Wall Y+, Baseline Mesh, Alpha=17 (M=0.17, Re=3.79E6) ….......................................... 42

Figure 36: Pressure Coefficient, Baseline Mesh, Alpha=17 (M=0.17, Re=3.79E6) ............................. 42

Figure 37: Velocity Contours, Baseline Mesh, Alpha=18, Transition SST Model (M=0.17, Re=3.79E6) .....43

Figure 38: Velocity Contours, Baseline Mesh, Alpha=18, S-A (M=0.17, Re=3.79E6) ..............................43

Figure 39: Velocity Contours, Alpha=18, sstkw (M=0.17, Re=3.79e6) ................................................43

Figure 40: Wall Shear Stress, Baseline Mesh, Alpha=18 (M=0.17, Re=3.79E6) .................................... 44

Figure 41: Truncated Wall Shear Stress Plot, Baseline Mesh, Alpha=18 ( $M=0.17, \mathrm{Re}=3.79 \mathrm{E} 6)$................44

Figure 42: Wall $\mathrm{Y}+$ Distribution, Baseline Mesh, Alpha=18 (M=0.17, $\mathrm{Re}=3.79 \mathrm{E6})$.................................45

Figure 43: Pressure Coefficient Distribution, Baseline Mesh, Alpha=18 ( $M=0.17, R e=3.79 E 6)$................45

Figure 44: NACA2412 Baseline, Course Mesh, 25,000 Nodes, Transition SST .....................................50

Figure 45: NACA2412 Medium (Ideal) Baseline Mesh, 100,000 Nodes, Transition SST .....................50

Figure 46: NACA 2412 Fine Baseline Mesh, 400,000 Nodes, Transition SST ...................................51

Figure 47: Course Mesh, 25K Nodes, Transition SST, Alpha=16.......................................................52

Figure 48: Medium Mesh (Ideal), 100K Nodes, Transition SST, Alpha=16 ….....................................53

Figure 49: Fine Mesh, 400K Nodes, Transition SST, Alpha=16 ..................................................... 53

Figure 50: NACA 2412 Spoiler Study Lift Coefficient, Alpha Results, 60\% LE Location.............................57

Figure 51: NACA 2412 Spoiler Study Lift Coefficient, Alpha results, 65\% LE location ...............................58

Figure 52: NACA 2412 Spoiler Study Lift Coefficient, Alpha results, 70\% LE location ..............................59

Figure 53: NACA 2421 Spoiler Study, CL/CD at 60\% LE location ........................................................60

Figure 54: NACA 2412 Spoiler Study, CL/CD, 65\% LE location .......................................................... 61

Figure 55: NACA 2412 Spoiler Study, CL/CD, 70\% LE location ............................................................ 61

Figure 56: Velocity streamlines 60\%, 65\% 70\% LE, Alpha=16, Spoiler deflection= 4, $M=0.17$, Transition

SST, Re=3.79E5

Figure 57: Velocity Magnitude Streamlines for Baseline, 60\%, 65\% \& 70\% LE, Spoiler Deflection =10 degrees, Alpha=16 degrees, Transition SST Model, Re=3.79E6

Figure 58: NACA 2412 Spoiler Study, Pressure Coefficient, Spoiler deflection = 4 degrees, Alpha=16 degrees, Transition SST Model, Re=3.79E6 
Figure 59: NACA2412 Spoiler Study, Pressure Coefficient, Spoiler deflection= 10 degrees, Alpha=16 degrees, Re=3.79E6, Transition SST Model .68

Figure 60: NACA 2412 Spoiler Study, Wall Shear Stress, Spoiler Deflection = 10 degrees, Alpha =16

degrees, Transition SST Model, Re=3.78E6

Figure 61: NACA 2412 Spoiler Study, Wall Shear Stress, Spoiler Deflection = 4 degrees, Alpha = 16

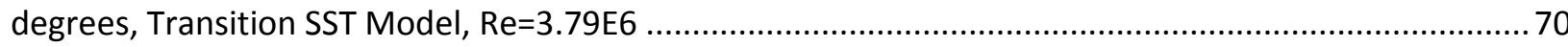

Figure 62: Velocity Magnitude Streamlines, Alpha=17 Degrees, Transistion SST Model, Re=3.79E6 ........74

Figure 63: Velocity Magnitude Streamlines, Alpha=18 Degrees, Transition SST Model, Re=3.79E6 .........75

Figure 64: Velocity Magnitude Streamlines, Alpha=19 Degrees, Transition SST Model, Re=3.79E6 .........76

Figure 65: Wall Shear Stress, Alpha=17 Degrees, Spoiler Deflection = 4 Degrees Transition SST Model,

$\mathrm{Re}=3.79 \mathrm{E} 6$

Figure 66: Wall Shear Stress, Alpha = 18 Degrees, Spoiler Deflection = 4 Degrees, Transition SST Model, $\operatorname{Re}=3.79 E 6$

Figure 67: Wall Shear Stress, Alpha=19 Degrees, Spoiler Deflection = 4 Degrees, Transition SST Model,

$\operatorname{Re}=3.79 E 6$ 79

Figure 68: Pressure Coefficient, Alpha=17 Degrees, Spoiler Deflection= 4 Degrees, Transition SST Model, $\operatorname{Re}=3.79 E 6$ .80

Figure 69: Pressure Coefficient, Alpha= 19 Degrees, Spoiler Deflection = 4 Degrees, Transition SST Model, $\mathrm{Re}=3.79 \mathrm{E} 6$ 81

Figure 70: Pressure Coefficient, Alpha=18 Degrees, Spoiler Deflection= 4 Degrees, Transition SST Model, $\operatorname{Re}=3.79 E 6$ .82

Figure 71: Mesh Layout of Domain and Fluid Region of Baseline NACA2412 airfoil section.................86

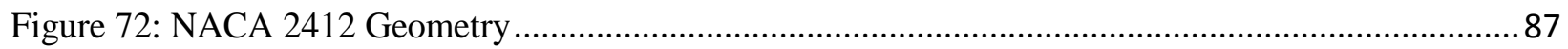

Figure 73: Baseline, Ideal Mesh, Farfield Distribution....................................................................... 90

Figure 74: Baseline, Ideal Mesh, NACA2412 Near wall Distribution ................................................91

Figure 75: NACA2412 Leading Edge View ................................................................................. 91

Figure 76: NACA2412 Trailing Edge View ................................................................................ 92

Figure 77: NACA2412 Airfoil-Spoiler Farfield Mesh Details ............................................................93

Figure 78: NACA2412 Airfoil-Spoiler Boundary Layer Mesh Details .................................................94

Figure 79: NACA2412 Airfoil-Spoiler Mesh Details ......................................................................... 94

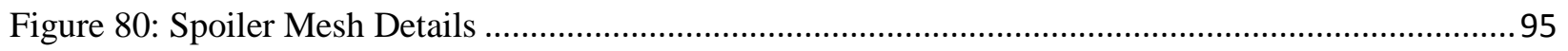

Figure 81: NACA2412 airfoil-Spoiler Farfield Overview …..............................................................99 
Figure 82: NACA2412-Spoiler Leading Edge View ..................................................................... 100

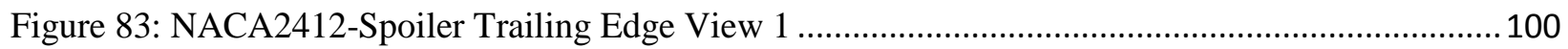

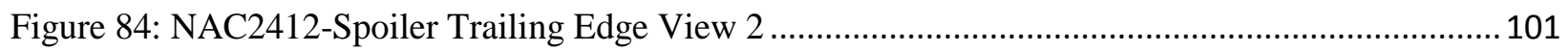

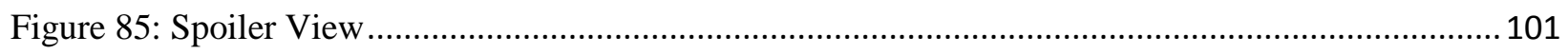

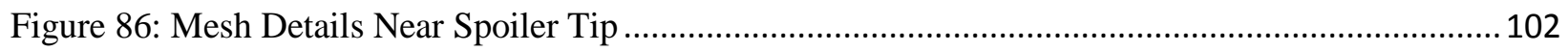

Figure 87: NACA 2412 Spoiler Study, Wall Y+, Spoiler Deflection = 4 degrees, Alpha = 16 Degrees,

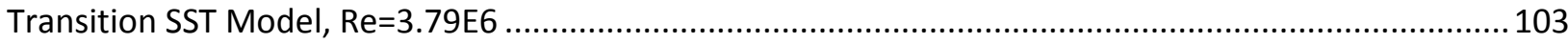

Figure 88: NACA 2412 Spoiler Study, Wall Y+, Spoiler Deflection = 10 degrees, Alpha = 16 Degrees,

Transition SST Model, Re=3.79E6 104

Figure 89:Pressure Coefficient, Alpha=17 degrees, Spoiler deflection=10 degrees, $M=0.17, \operatorname{Re}=3.79 E 6$,

Transition SST. 105

Figure 90: Variation of Velocity Coloured Streamlines with Upper Surface Location, Spoiler deflection=10 degrees, Alpha=17, M=0.17, Re=3.79E6 .106

Figure 91: Wall shear stress plot, Alpha=17, Spoiler Deflection=10, $M=0.17, \mathrm{Re}=3.79 \mathrm{E} 6$, Transition SST 107

Figure 92: Wall $Y+$, Alpha=17, Spoiler Deflection=4, $M=0.17, \mathrm{Re}=3.79 E 6$, Transition SST ..................... 108

Figure 93: Wall $Y+$, Alpha=17, Spoiler Deflection=10, $M=0.17, \mathrm{Re}=3.79 E 6$, Transition SST .................... 109

Figure 94: Pressure Coefficient, Alpha=18, Spoiler Deflection=10, $M=0.17, \mathrm{Re}=3.79 E 6$, Transition SST 111

Figure 95: Variation of Velocity coloured streamlines with Upper surface location, Alpha=18, Spoiler deflection=10, M=0.17, Re=3.79E6, Transition SST Model.

Figure 96: Wall Shear Stress, Alpha=18, Spoiler Deflection=10, $M=0.17, \mathrm{Re}=3.79 E 6$, Transition SST Model

Figure 97: Wall $Y+$, Alpha=18, Spoiler Deflection=10, M=0.17, Re=3.79E6, Transition SST Model ..........114

Figure 98:Wall $Y+$, Alpha=18, Spoiler Deflection=4, M=0.17, Re=3.79E6, Transition SST Model ............115

Figure 99: Pressure Coefficient, Alpha=19, Spoiler Deflection=10, $M=0.17, R e=3.79 E 6$, Transition SST

Model

Figure 100: Variation of Velocity coloured Streamlines with Upper surface location, Alpha=19, Spoiler

Deflection=10, M=0.17, Re=3.79E6, Transition SST Model .118

Figure 101: Wall Shear Stress, Alpha=19, Spoiler Deflection=10, M=0.17, Re=3.79E6, Transition SST

Model 119

Figure 102: Wall Y+, Alpha=19, Spoiler Deflection=10, $M=0.17, R e=3.79 E 6$, Transition SST Model........120

Figure 103: Wall Y+, Alpha=19, Spoiler Deflection=4, $M=0.17, \mathrm{Re}=3.79 E 6$, Transition SST Model..........121 
Figure 104: Pressure Coefficient, Alpha=20, Spoiler Deflection=4, $M=0.17, \operatorname{Re}=3.79 E 6$, Transition SST

Model

Figure 105: Variation of Velocity coloured Streamlines with Upper surface Location, Alpha=20, Spoiler deflection=4, M=0.17, Re=3.79E6, Transition SST . . . .

Figure 106: Wall Shear Stress, Alpha=20, Spoiler Deflection=4, $M=0.17, \mathrm{Re}=3.79 E 6$, Transition SST Model

Figure 107: Wall Y+, Alpha=20, Spoiler Deflection=4, $M=0.17$, Re=3.79E6, Transition SST Model..........126

Figure 108: Pressure Coefficient, Alpha=21, Spoiler Deflection=4, $M=0.17, \operatorname{Re}=3.79 E 6 \ldots \ldots \ldots \ldots \ldots \ldots \ldots . . . . . . . . . .127$

Figure 109: Velocity coloured streamlines, Alpha=21, Spoiler Deflection $=4, M=0.17, \operatorname{Re}=3.79 E 6 \ldots \ldots . .129$

Figure 110:Wall Shear Stress, Alpha=21, Spoiler Deflection=4, M=0.17, Re=3.79E6 ......................... 130

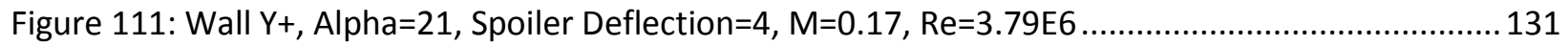

Figure 112: Pressure Coefficient, Alpha=23, Spoiler Deflection =4, $M=0.17, \operatorname{Re}=3.79 E 6 \ldots \ldots \ldots \ldots \ldots \ldots \ldots . . . . . . . . . .132$

Figure 113: Velocity coloured Streamlines, Alpha=23, Spoiler Deflection=4, $M=0.17, \operatorname{Re}=3.79 E 6 \ldots \ldots \ldots .133$

Figure 114: Spoiler Study, Wall Shear Stress, Alpha = 23, Spoiler Deflection $=4, M=0.17, \operatorname{Re}=3.79 E 6$,

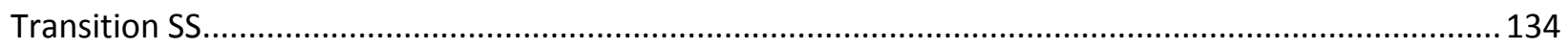

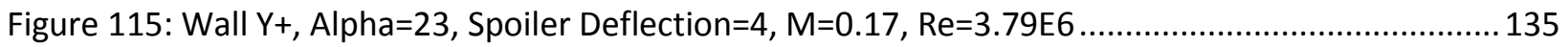




\section{List of Symbols}

\begin{tabular}{|c|c|}
\hline$a$ & gas velocity $[\mathrm{m} / \mathrm{s}]$ \\
\hline c & chord length $[\mathrm{m}]$ \\
\hline cd & drag coefficient \\
\hline cl & lift coefficient \\
\hline $\mathbf{c p}$ & pressure coefficient \\
\hline$f$ & body force components \\
\hline$L E$ & leading edge \\
\hline$M$ & Mach number \\
\hline $\mathbf{p}$ & pressure $[\mathrm{Pa}]$ \\
\hline$q$ & heat flux \\
\hline $\boldsymbol{R}$ & gas constant, 287 [J/kgK] \\
\hline$S$ & Sutherland's constant, $110.56[\mathrm{~K}]$ \\
\hline$T E$ & trailing edge \\
\hline$T_{o}$ & reference temperature, $273.11[\mathrm{~K}]$ \\
\hline$T$ & temperature $[\mathrm{K}]$ \\
\hline $\boldsymbol{V}$ & velocity $[\mathrm{m} / \mathrm{s}]$ \\
\hline$\alpha$ & alpha, angle of attack [degrees] \\
\hline$\varepsilon$ & internal energy per unit mass \\
\hline $\boldsymbol{\kappa}$ & von Kármán constant \\
\hline$\rho$ & density $\left[\mathrm{kg} / \mathrm{m}^{3}\right]$ \\
\hline$\delta$ & spoiler deflection angle [degrees] \\
\hline $\boldsymbol{\mu}$ & viscosity $[\mathrm{kg} / \mathrm{ms}]$ \\
\hline$\mu_{o}$ & reference gas viscosity, $1.716 \mathrm{E}-05[\mathrm{~kg} / \mathrm{ms}]$ \\
\hline$\tau$ & viscous stress tensor \\
\hline$v_{T}$ & turbulent viscosity $[\mathrm{kg} / \mathrm{ms}]$ \\
\hline $\boldsymbol{\gamma}$ & air gas constant ratio, 1.4 \\
\hline
\end{tabular}




\section{List of Appendices}

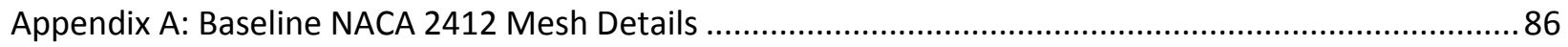

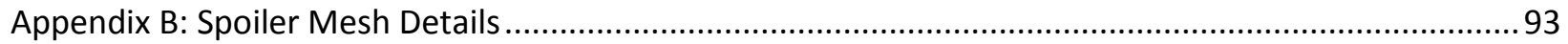

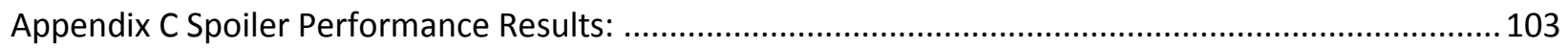

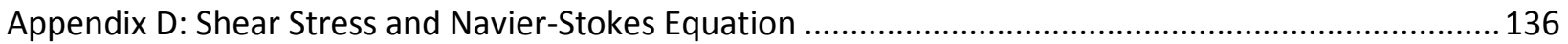




\section{Chapter 1}

\section{Introduction}

\subsection{Motivation}

In the field of aerodynamics one of the primary demands in design is improving aerodynamic efficiency. Often the strategy employs producing clever devices that are utilized to improve lift performance and/or decrease drag. With advancing technology, Computational Fluid Dynamics (CFD) has provided a platform for researchers to investigate such techniques within complicated flow regimes; thereby significantly expanding the scope of aerodynamic research, which otherwise would be extremely difficult and costly using conventional wind tunnels testing facilities.

The objective of this thesis is to conduct a two dimensional CFD analysis of wing-spoiler effectiveness in retarding stall effects on a NACA2412 airfoil section. Spoilers, as the name suggests, are essentially operated to function as 'lift reducing' devices. Commonly located on the upper wing surfaces, the spoilers are deployed for the purposes of creating an early onset of flow separation to aide as aircraft flight controls (i.e. upon descent or the rolling or banking of an aircraft (among other functions)). [1]

In the past, researchers have invested much time in understanding aerodynamics surrounding upper surface wing spoilers. Investigators, Lee \& Bodapti at Stanford University conducted an experimental study in which a Boeing transport airfoil section was fitted in their low speed wind tunnel test section $(14.5 \times 14.5 \mathrm{~cm})$ with a spoiler section connected near the trailing edge of the upper surface (details on the spoiler specifications were not provided). The study was aimed at 
understanding the surface pressure distribution forward and aft of the spoiler section. Angle of attack values between -10 to 12 degrees were varied for spoiler deflections of $0,15,30$ and 60 degrees at a Reynolds number of 2.8E5, with a corresponding free stream velocity of approximately $20 \mathrm{~m} / \mathrm{s}$. The spoiler length was measured to be approximately $15.54 \%$ of the airfoil chord length (1 m chord in total) and was fixed at $73 \%$ leading edge chord location. Typical results for pressure coefficient on the upper and lower surface regions can be seen in Figure 1 for the alpha case of 2 degrees, on the following page. Figure 1 shows the static pressure on the upper surface suction side decrease with increasing spoiler deflections for points forward of the spoiler location. In contrast, the trend in static pressure was seen to spike for points in the aft region of the spoiler across all spoiler deflections tested. This trend was also seen on the lower surface; however there in comparison there was a slow increase in static pressure near the trailing edge region of the airfoil upper surface.
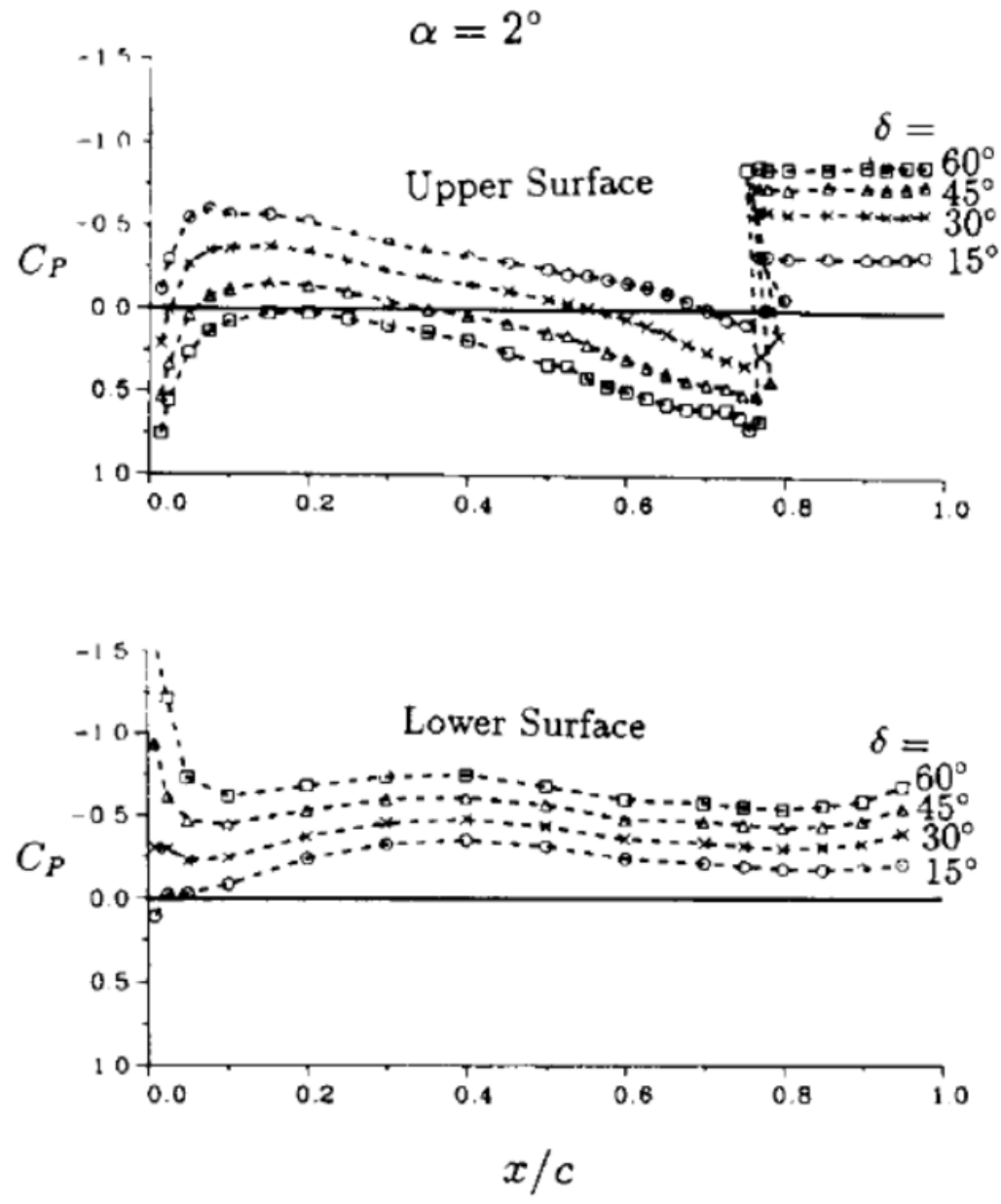

Figure 1: Pressure Distribution on the Upper surface across various spoiler deflections [1] 
The overall gain in static pressure could not offset the considerable decrease in the pressure distribution seen from the leading edge to the forward section of the spoiler region. This finding was further demonstrated through a display of the alpha-lift coefficient plot across all spoiler deflections (Figure 2). This plot showed a decrease in lift with increasing spoiler deflection across all alpha tested (-10 to 12 degrees). Therefore, from these results it is quite evident that implementation of the spoiler in-fact hinders lift performance. However it is interesting to note that the values of alpha tested were in the pre-stall region of the lift-alpha plot seen in Figure 2 [1].

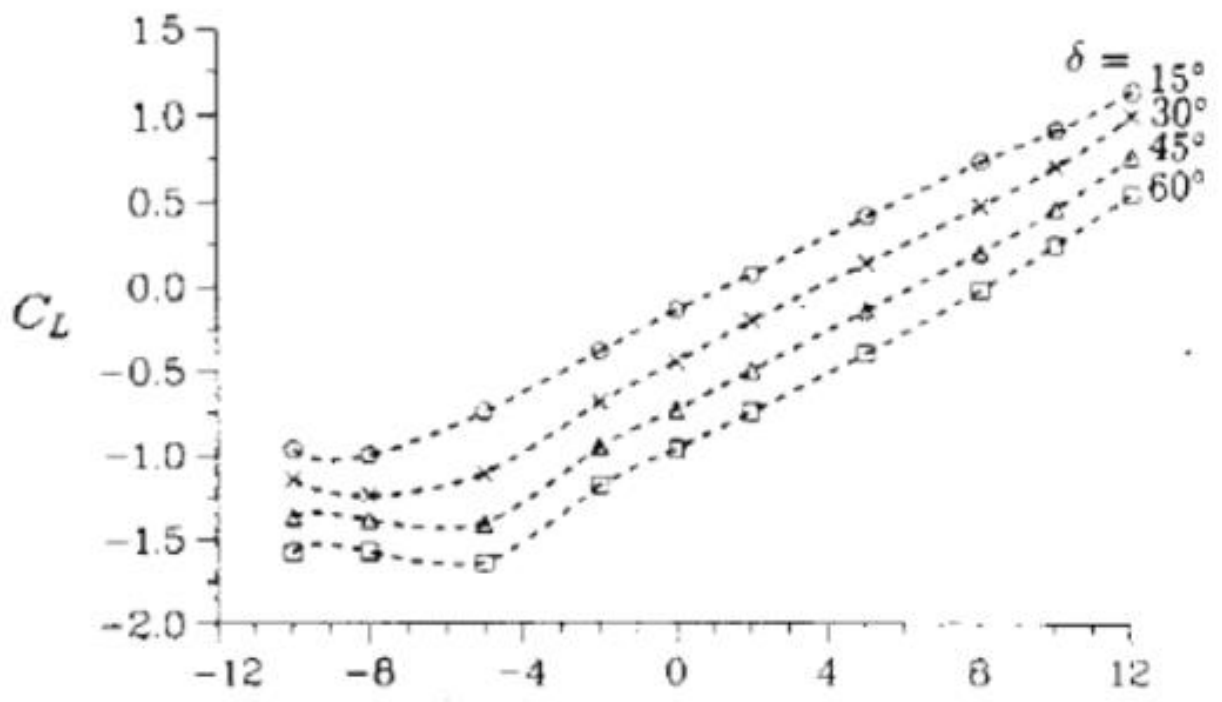

Figure 2: Lift-alpha plot for various spoiler deflections [1]

In another study, an investigation was conducted on the interaction of airfoil-spoiler-flap combination on lift performance. Investigators Shabibi et al. carried out experiments using the NACA 4424 airfoil section as there baseline of choice. Similar to Lee \& Bodapati, spoiler deflections of $0,15,30,45$ and 60 were tested for values of alpha within the range of -6 to +6 degrees. Lift and drag coefficient plots, Figure 3 shown below, for the airfoil-spoiler combination show depreciation in the lift characteristic with increasing spoiler deflections. [2]. 


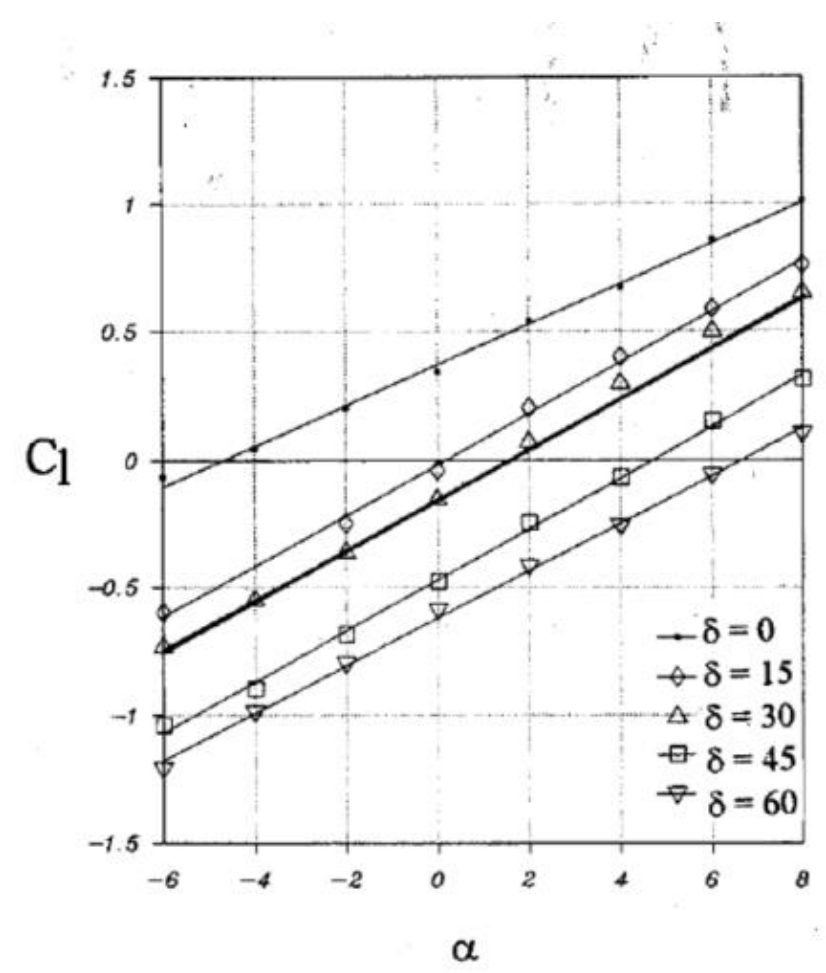

Figure 3: Lift-alpha curve for NACA4424-

spoiler section [2]

The commonality between the scholarly articles mentioned above are the low pitch angles used to test spoiler lift performance. In other studies, for example, Mbdelrahman et al. [3] conducted an experimental study on a NACA 23012 airfoil section in which inboard spoilers were tested in both symmetrical and non-symmetrical combinations for the purpose of examining aerodynamic loads such as the lift, drag and moment coefficients in the presence of wind shear. The spoilers were fixed $20 \%$ from the trailing edge of the wing and lift performance was measured across both high and low values of alpha (between 0-16 degrees). According to the results, the most significant loss of lift was observed for low angles of attack; whereas the effects were less pronounced at high angle of attack. According to the article, this finding was in part due to the leading edge stall behaviour of the NACA 23012 airfoil section. Therefore, at high alpha, the separation bubble was situated ahead in location of the spoiler position; hence spoiler's influence was considered non-significant. Thus, the results presented in this paper provided insight of how the spoiler behaves with an airfoil section that has a naturally occurring leading edge stall. That being said, considering spoiler lift performance at high alpha, but with an airfoil section that has 
a clean trailing edge stall was one of the main influencing drivers that motivated the start of this research. [3].

Similarly, research presented by Pfeiffer and Zumwalt [4] provided performance results for two airfoil-spoiler sections: a Boeing airfoil (11.3\% thickness) with the spoiler located $73.3 \%$ leading edge of chord and LS(1)-0413 (13\% thickness) with the spoiler hinged at $77.5 \%$ leading edge. Unlike previous results, a computational model was developed to closely simulate the experimental results studied in the past for two airfoil sections mentioned. Spoiler deflections of 2.5 to 90 degrees were varied across alpha values between 0 to 16 degrees. The lift-alpha plots for both airfoil sections can be seen in Figure 4 and Figure 5 seen below. Upon observation of the results lift degredation becomes apparent across the spoiler deflections tested. However, also apparent is the maximum alpha angle tested, which in both cases appears to be at 16 degree alpha, in the pre-stall region. [4]

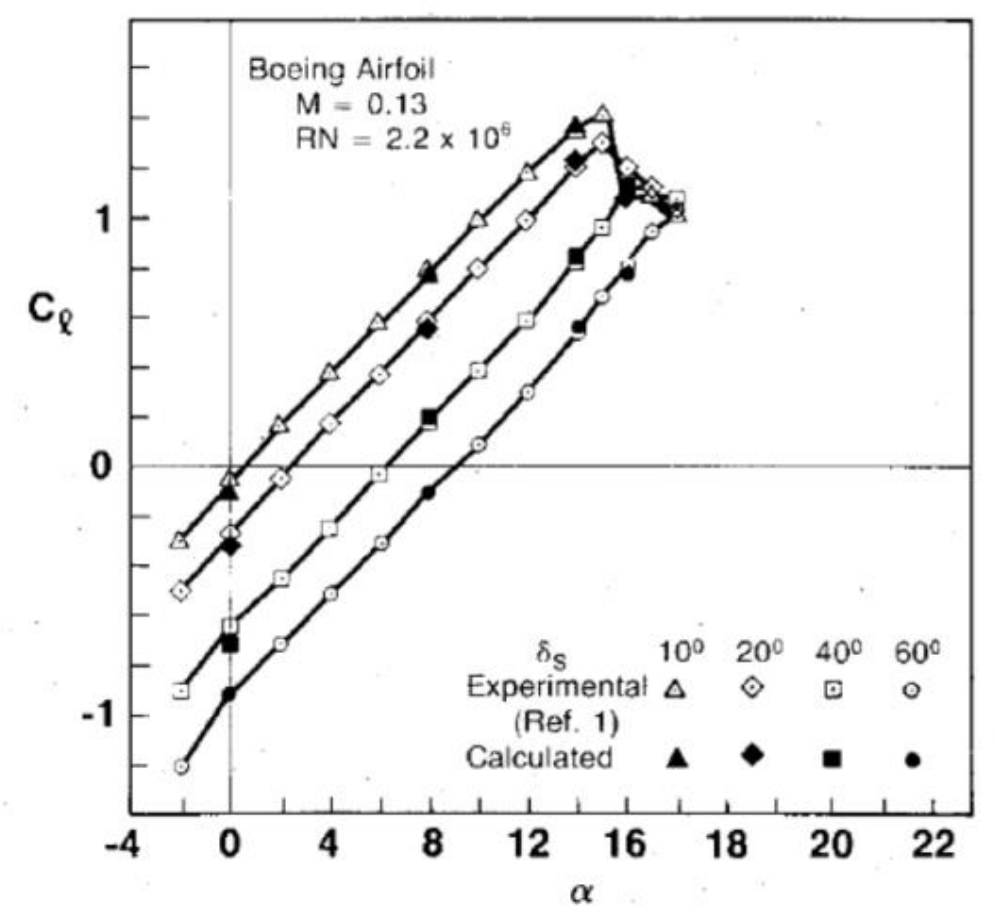

Figure 4: Lift-alpha curve for Boeing airfoilspoiler (73.3\%LE) [4] 


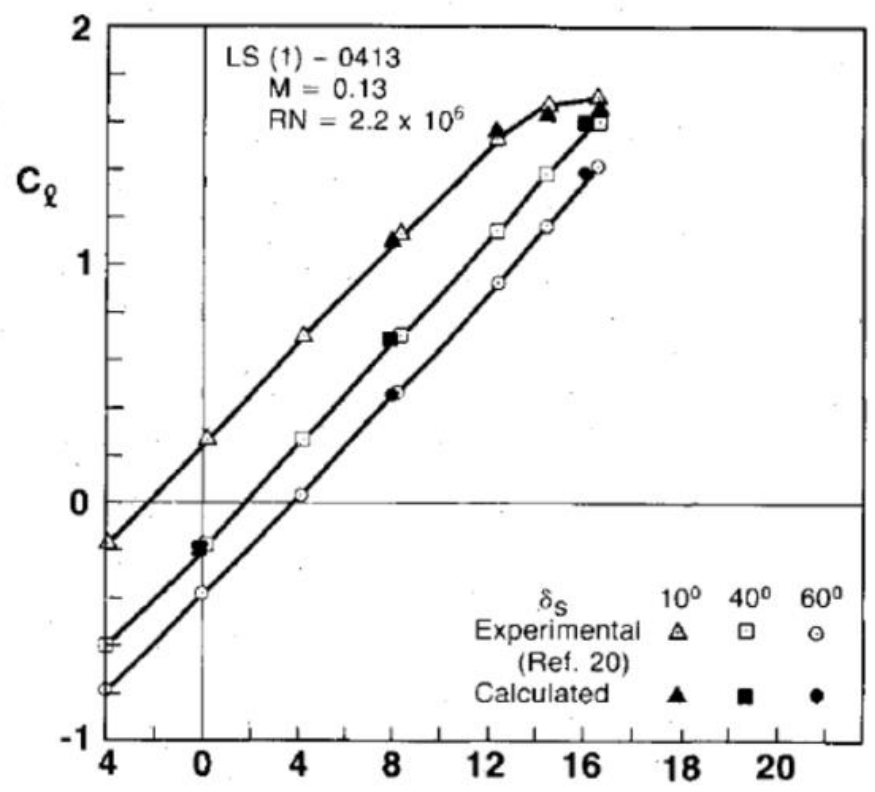

Figure 5: Lift-alpha plot of LS(1)-0413 airfoil-spoiler (77.5\%LE) [4]

In another paper, researchers Consigny et al. [5] devised a procedure that utilized an airfoil section, with $16 \%$ thickness and $0.18[\mathrm{~m}]$ chord length connected to a spoiler section $(0.15 \mathrm{c}$ in length) at $0.52 \mathrm{x} / \mathrm{c}$ location on the upper airfoil surface. The study's focus was to analyze both the pressure distribution and aerodynamic characteristics at an alpha of zero degrees under three flow Mach variations of 0.30- 0.80. Spoiler deflections of 0-20 degrees were analyzed. Similar to the results seen previously, with increasing spoiler deflections, the spoiler was shown to effectively increase the pressure distribution aft of its location, whereas forward was seen to suffer a decrease in pressure. These results can be seen on the following page. 


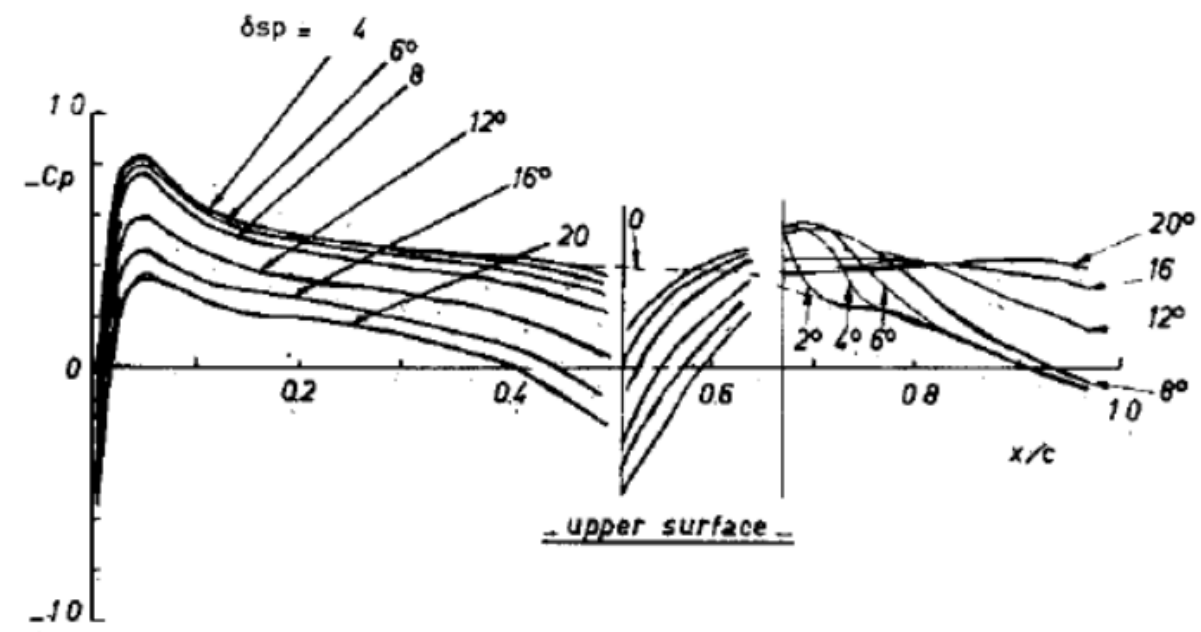

Figure 6: Variation in Cp with changing Spoiler deflection [5]

Typical of results collected in previous papers, Consigny et al. had seen an overall loss in lift coefficient for this angle of attack at zero degrees. They attribute the loss seen to an insufficient gain in back pressure (aft of the spoiler) compared to loss in pressure seen upstream of the spoiler position. Therefore, the effects of spoiler deflection, although seen to have detrimental effects on lift performance in cases for values of alpha in the pre-stall region, still are not fully understood in the post-stall region, which is ultimately the main focus of this research. [5]

\subsection{Spoiler Lift Capability}

Much research has been dedicated to utilizing wing spoilers as a means to reduce the effects of wake vortex hazard between two trailing aircraft. Studies have shown that oscillating the spoiler in such a way can create a counteracting vortex whose effects negatively cancel the wing tip vortices.

In a study conducted by Yeung et al. [6] at Nanyang University, Singapore, the NACA 0012 airfoil section was utilized to study unsteady aerodynamics behind the aft section of a spoiler located at $70 \%$ chord; having a length of $10 \%$ chord. The study was centered at providing a solution to alleviate the initial increase in lift and decrease in pitching moment that the spoiler section was observed to endure during descent. According to the article, when the spoiler is 
initially extended at high speeds, a vortex develops near the spoiler tip which induces an increase in lift. Once the spoiler is deflected to its maximum setting, this incremental increase in lift is observed to diminish as the vortex separates from the tip. According to the article, the increase in lift "can be as large at 50\% of the net change in lift" [7]. Although, the primary focus of the study was to eliminate any incremental increases in the lift observed, as these effects would hinder aircraft landing descents, the study also provided further insight into the potential lift producing capabilities that the spoiler may alternatively function to work as. Particularly, in another derivative, Yeung et al. provided a computational model to better resolve the physics in the aft region of a base-vented spoiler connection to the upper airfoil surface. The idea behind the base-vented device was to create a counter rotating vortex that would essentially eliminate the initial increase in lift observed. Although, not-significant for the purpose of this research, the article provided key information on the lift increase seen. The spoiler in the study was considered to be an oscillating device and essentially was analyzed between two cases: deflection from 10 to 30 degrees at an alpha of 12 degrees and deflection from 10-50 degrees at an alpha of 0 degrees. Spoiler tip speeds were taken as a non-dimensional ratio of velocity and according to the article were computed as 0.0174 and 0.370 . Results were shown to capture the incremental increase in lift, and as an example, results for an alpha of 0 degrees with a spoiler tip speed of 0.0174 is shown below, along with the flow patterns seen for visual purposes:

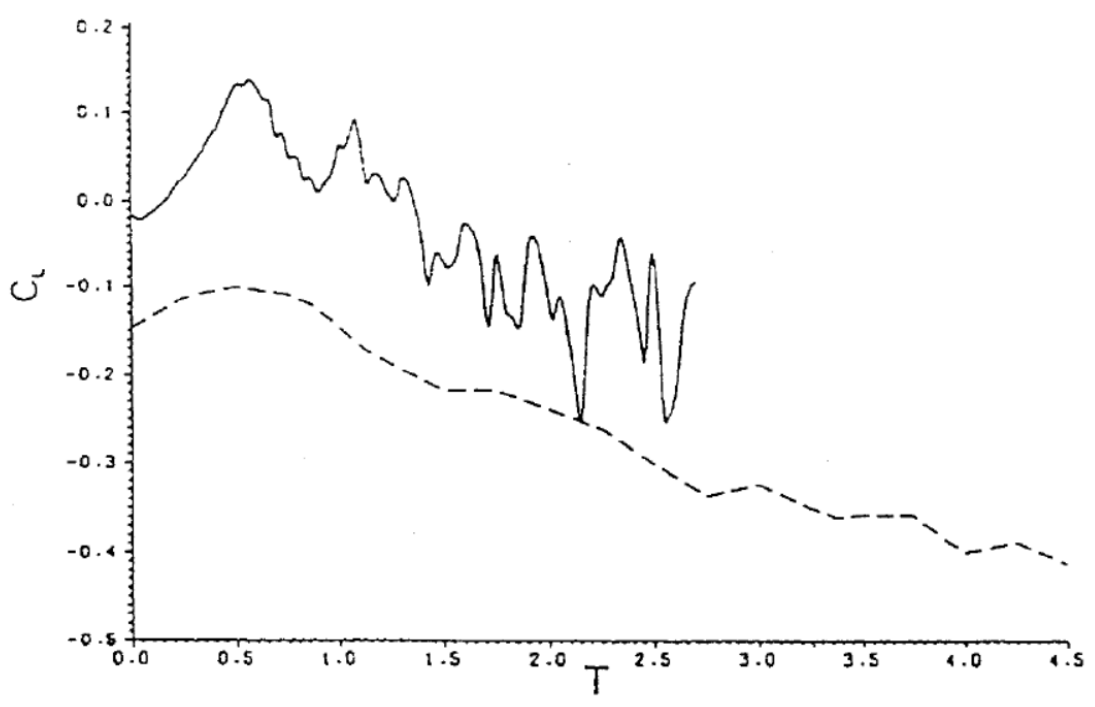

Figure 7: Lift variation from oscillating spoiler [6] 

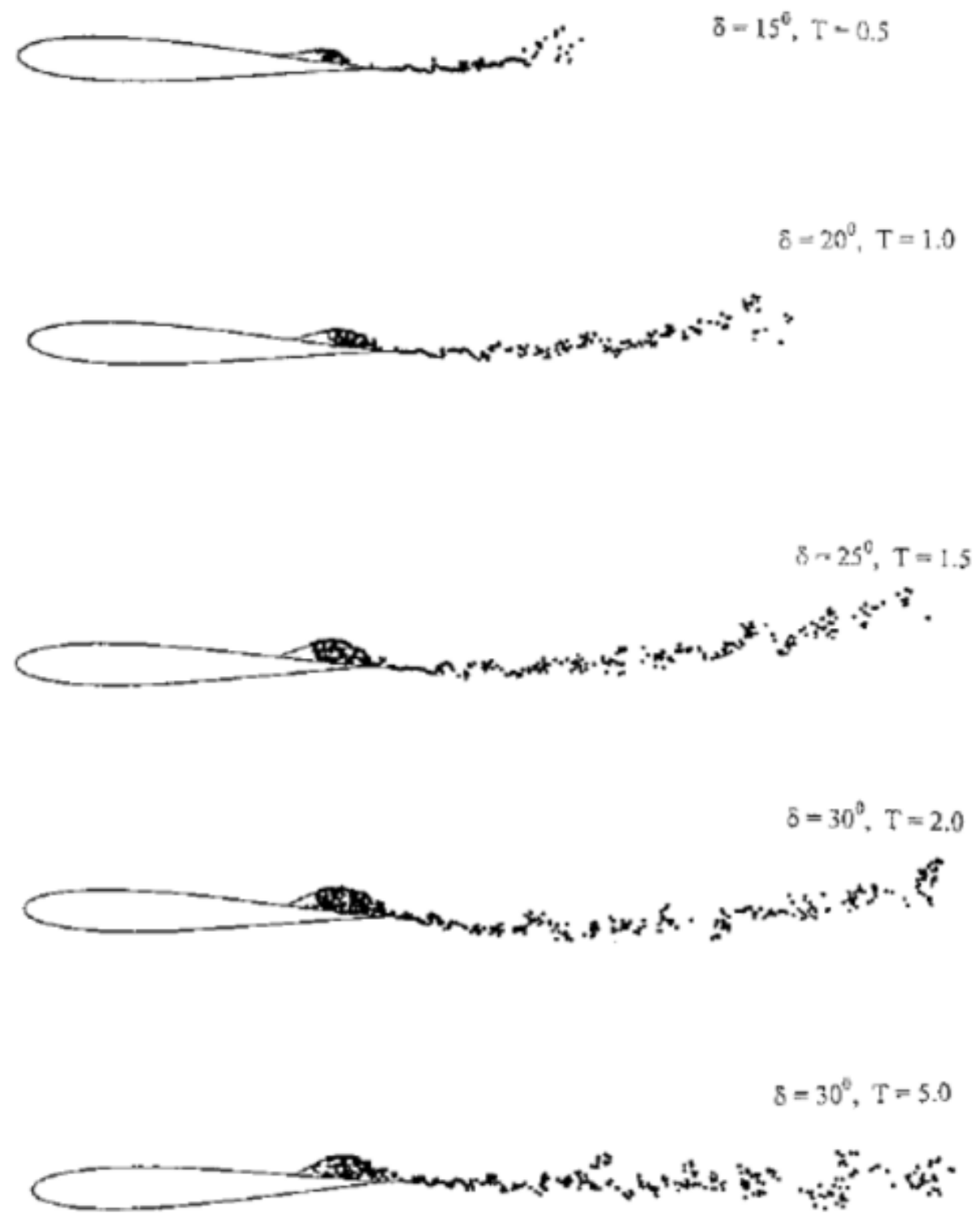

Figure 8: Flow Patterns seen aft spoiler [6]

In Figure 7 (on the previous page) the non-dimensional time unit represents the release and end point in spoiler deployment. It is interesting to note the initial increase in lift observed from moving the spoiler at 10 degrees through to 50 degrees. Also note the strength of the vortices begin shed in Figure 8 as the spoiler is deflected. According to the plot, the higher the spoiler deflection the stronger the vortices shed; it also shows the adverse lift seen occurred at a time stamp of around 0.5 , corresponding to a spoiler deflection of 15 degrees. Nonetheless, even 
though the results describe an oscillatory spoiler section, the findings do show the potential of producing a positive increase in lift due the addition of the spoiler geometry. Therefore, from the results thus seen, further analyzing small angle spoiler deflections with the use of a rigidly attached spoiler (rather than an oscillatory one) sparked some interest to continue this research on adverse lift generation and spoiler performance. [6]

Furthermore, researchers Choi et al. [8] from the Korean Aerospace Research Institute providing additional finding for the adverse lift experienced by the oscillating spoiler using a Navier-Stokes based solver. Three turbulence models were used: Baldwin-Lomax k-w, Wilcox k-w and the shear stress transport (SST) model were used. A C-grid mesh was used, $261 \times 51$, and narrowing in on one interesting case taken from the study where the spoiler was situated at $0.70 \mathrm{c}$ chord position and the solution was converged via the SST turbulence model results for the streamlines are shown in Figure 9 to the right. As can be seen in Figure 9, increasing the spoiler deflection from 13.2 to 51.2 degrees showed a positive increase in the lift coefficient corresponding to points on the lift coefficienet curve labelled 1 to 3 , shown at the top end of Figure 9. Therefore, similar to research mentioned earlier with oscillatory spoiler performance, there is an initial adverse lift seen which may act as a potential mechanism in alleviating or mitigating stall effects. [8]
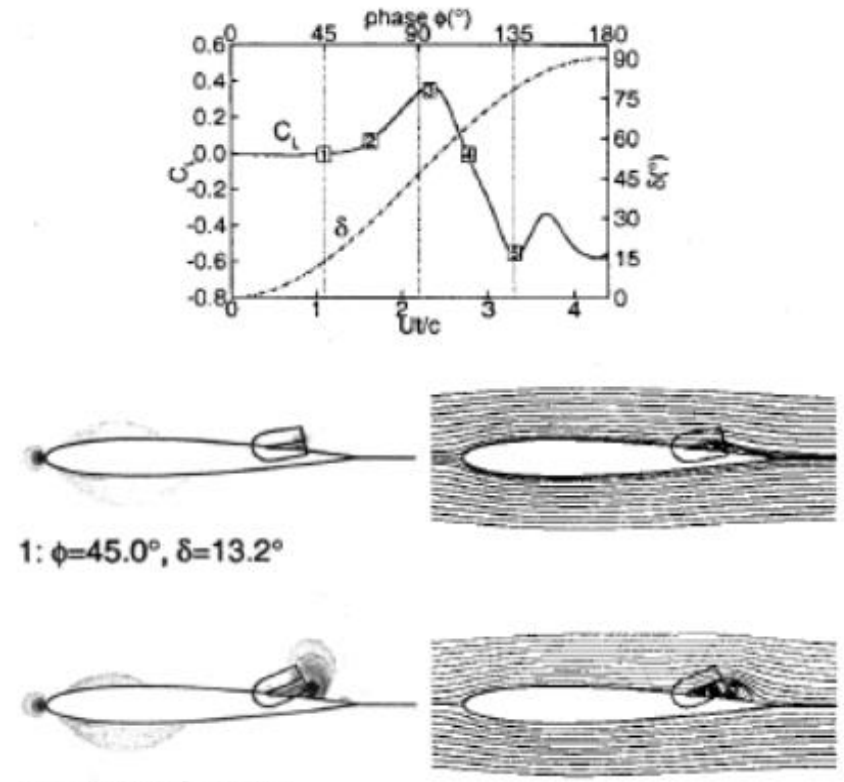

2: $\phi=67.5^{\circ}, 8=27.8^{\circ}$

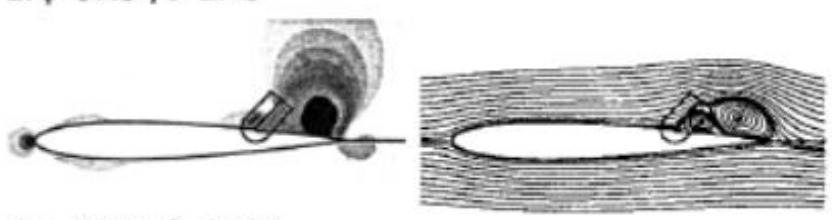

3: $\phi=98.0^{\circ}, \delta=51.2^{\circ}$

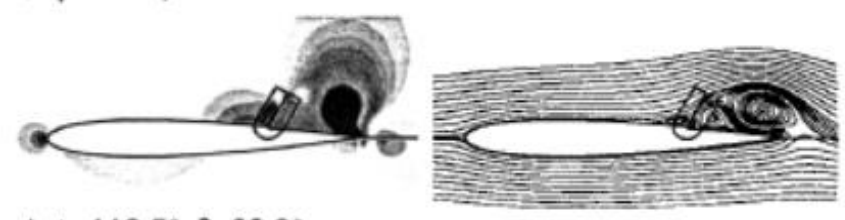

$4: \phi=112.5^{\circ}, \delta=62.2^{\circ}$

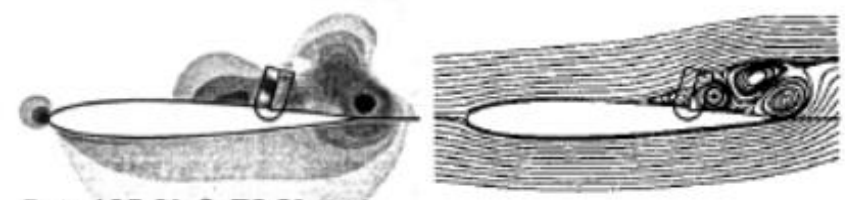

$5: \phi=135.0^{\circ}, \delta=76.8^{\circ}$

Figure 9: Pressure and Streamline Distribution for various spoiler deflections [8] 
A semi-empirical method was suggested by Jones et al. [9] in which a plain spoiler (rigidly attached to the upper airfoil surface) was experimentally tested for spoiler lift increase (a term that was referred to as the Lift Effectiveness parameter) commonly seen in gust alleviation studies. The study investigated the independent effects of airfoil-spoiler lift effectiveness against various geometrical parameters such as span-wise location, aspect ratio, and taper ratio amongst many others against aerodynamic characteristics of angle of attack and chord-wise location. Experimental data collected from previous wing-spoiler studies were utilized; however the exact details of the wing-spoiler specification were not revealed. A set of basic values for the parameters mentioned were provided as the baseline in which a correction factor representing the ratio of the experimentally computed lift effectiveness (i.e. spoiler length) was divided by the baseline basic value. When interrogating a range of values for a particular parameter, the researchers suggested the correction factors may be utilized for predictive purposes of lift effectiveness within the range limits. The study provided interesting results for how the lift effectiveness varied across the various chord-wise locations tested at a range of Mach values. A key plot for these results taken from the paper revealed the following Figure shown below. [9]

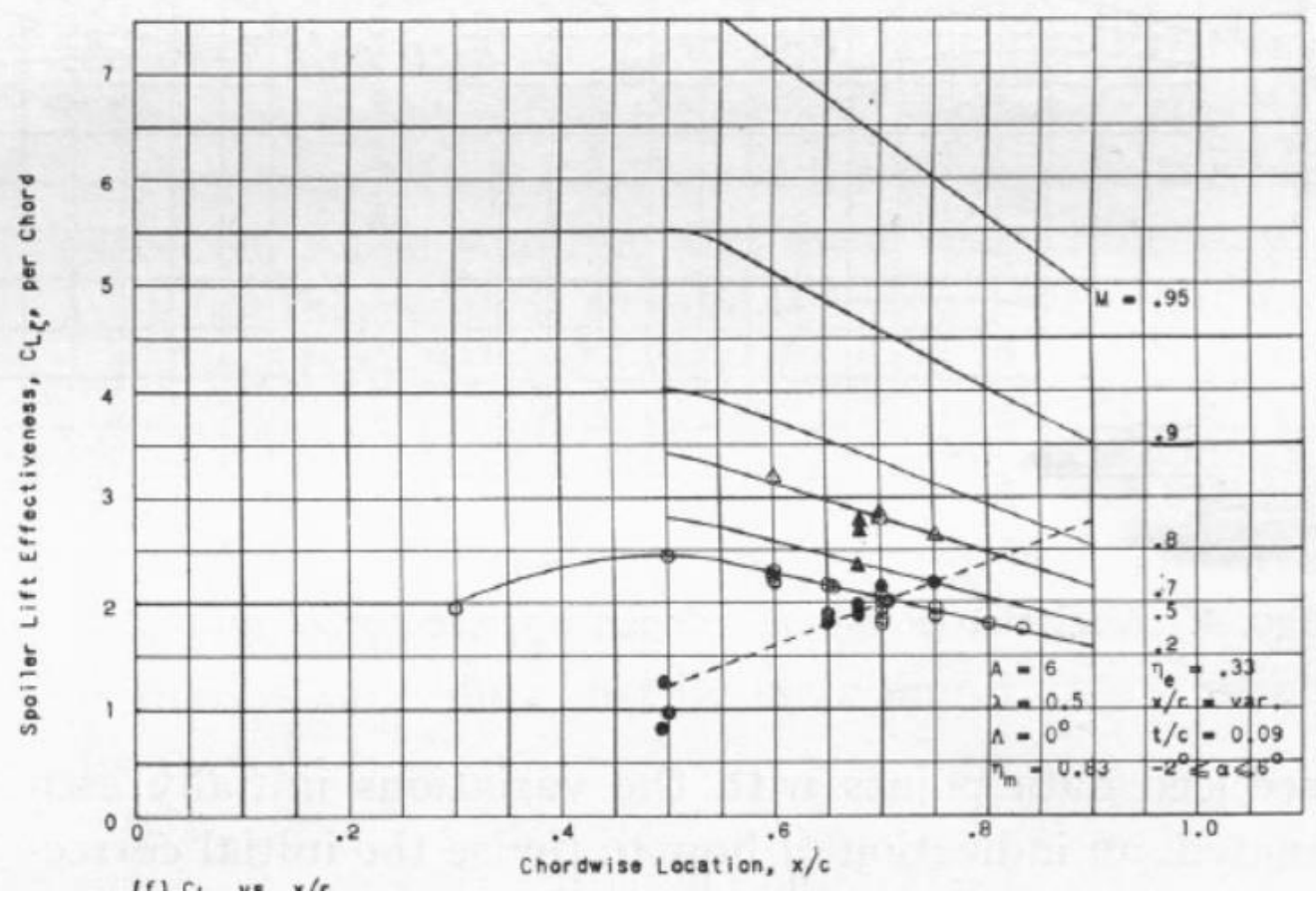

Figure 10: Lift Effectiveness vs. Chord Location with Mach [9] 
The plot shows the variation in spoiler lift effectiveness with chord-wise location. The data points seen on the graph correspond to data points collected from three dimensional wing spoiler configuration which were then modified to correspond to the basic values suggested by the researchers. According to the results, there is a strong clustering of data points near the 0.6-0.7 chord-wise location that shows a positive gain in lift effectiveness. Nonetheless, this paper had provided additional insight of not only a spoiler ability to produce positive lift, but also that there may be an ideal location along the chord that maximizes these effects with in the Mach range of subsonic speeds.[9]

The most promising results involving fixed airfoil-spoiler configurations and lift effectiveness in a study conducted by researchers Johnston and Gopalarathnam [10]. Wind tunnel tests were carried out at North Carolina State University at a free stream velocity of $30 \mathrm{~m} / \mathrm{s}$ at a Reynolds number of 4.0E5. The airfoil section used had a $12 \%$ cambered thickness and was $30.5 \mathrm{~cm}$ in chord length. Spoiler deflections between 15-90 degrees were tested for alpha values between 525 degrees. The spoiler size was measured to be $20 \%$ of the airfoil chord, and was fixed at $60 \%$ leading edge location on the upper surface of the airfoil section. Results for fixed spoiler study were found to be the following Figure:

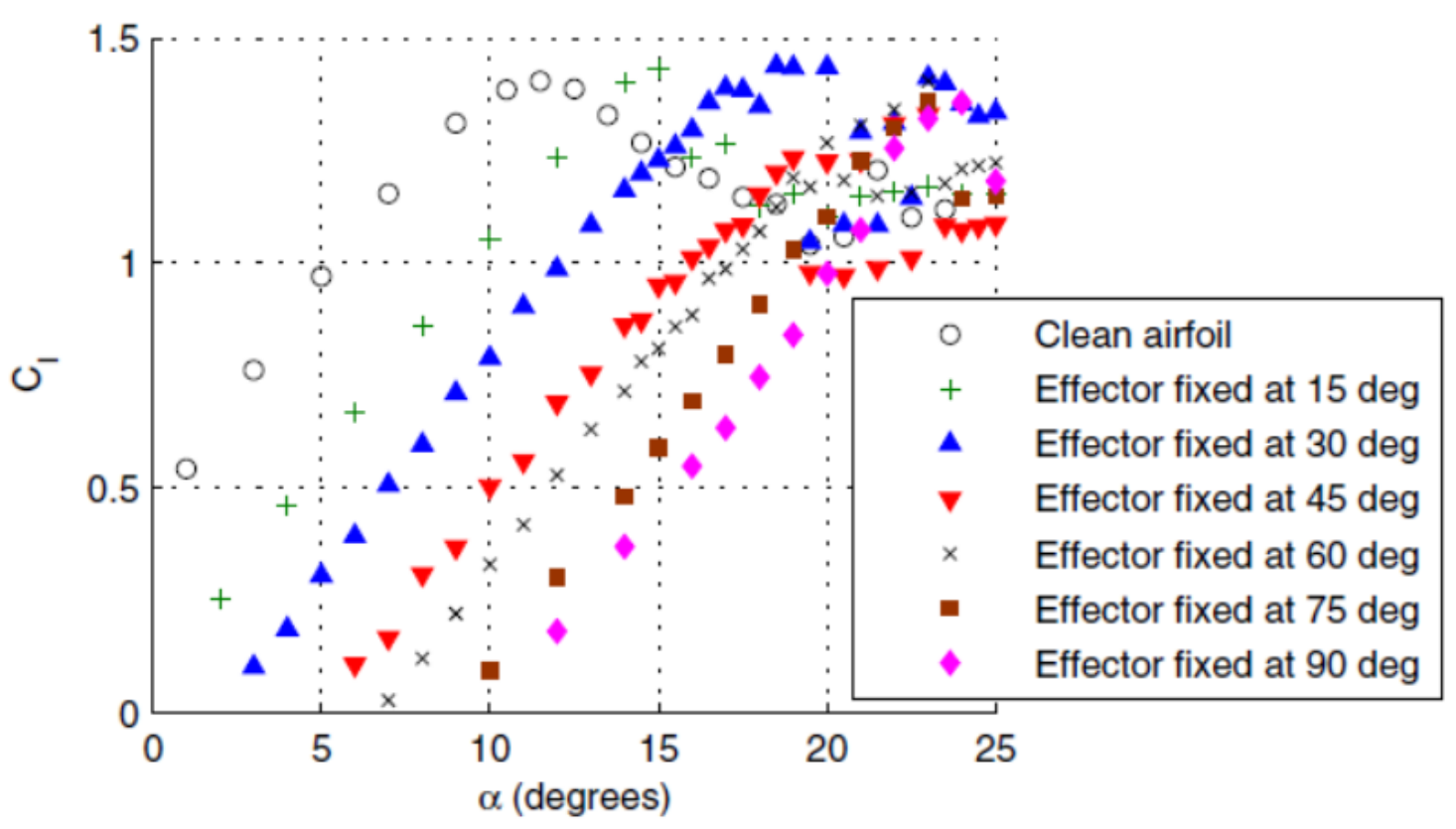

Figure 11: Cl-alpha curve for fixed spoiler deflection [10] 
As shown in Figure 11 increasing the spoiler deflection from 15-30 degrees has pushed the stall point further to the right. The higher the spoiler is deflected the greater the potential to produce lift at high alpha values in comparison to the clean airfoil section. Also evident is the detrimental effects the spoiler has for $\alpha$ cases prior to stall. In this scenario the spoiler, as suggested in the previous studies presented in this report, acts as a lift reducing device and actually hinders the flow further. This is seen in Figure 11 as the slope of the $\mathrm{Cl}-\alpha$ curve prior to stall shift further to the right, away from the points collected from the clean study. An example in the paper was provided of the pressure distribution seen at 18 degrees alpha for the spoiler deflection of 30 degrees in comparison to the clean case, as shown below:

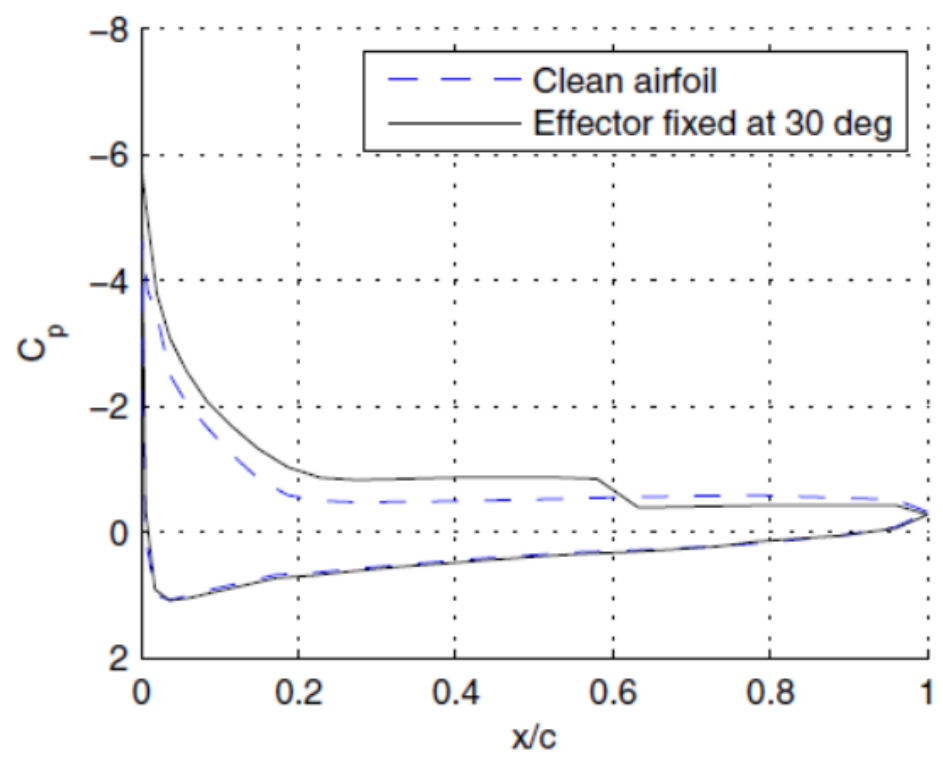

Figure 12: Pressure Distribution for Effector fixed at 30 degrees [10]

Post-analysis of Figure 12 reveals that the majority of lift produced culminated from the pressure distribution forward of the spoiler section (situated at $60 \% \mathrm{x} / \mathrm{c}$ ). The study does mention the small loss of lift due to an increase in pressure aft of the spoiler section on the upper surface which does not offset the overall increase in lift seen forward of the spoiler location.

In another paper, Bramesfeld and Maughmer [11] conducted wind tunnel experiments using a low speed Wind Tunnel at Pennsylvania State University. A S824 airfoil was used with a 17.7\% thickness and $45.6 \mathrm{~cm}$ chord length. The upper surface spoilers were approximately $9 \%$ chord length and the collected corresponded to a Reynolds Number of 1 million. Values for the pressure distribution were recorded for a single spoiler located at $55 \%$ and $86 \%$ upper surface 
chord location, along with the effects of having double effectors located at 70 and $86 \%$ chord. As show in Figure 13, the deployments of a single spoiler (effector) at 9 degrees, 86\% chord location had shown the maximum lift coefficient correspond to an alpha of 16 degrees compared to the baseline stall alpha of 14 , represented by square symbols in the figure below (note the moment coefficient is also shown).

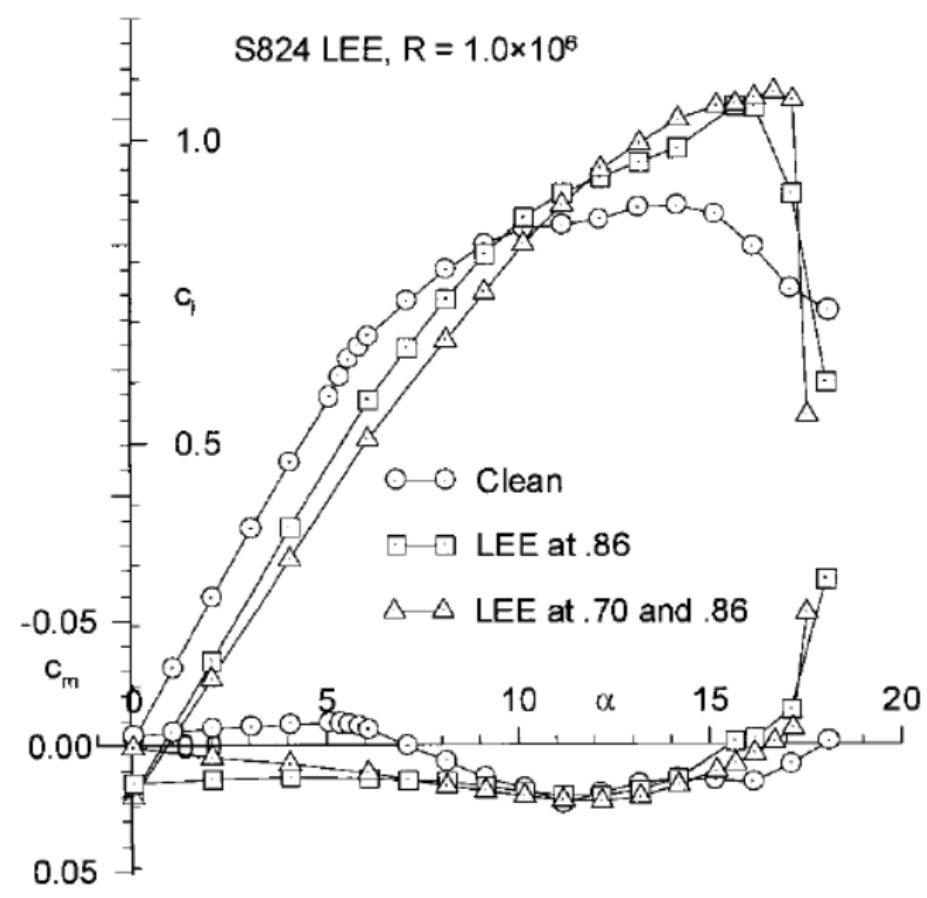

Figure 13: Lift Coefficient, S824, Reynolds Number= 1 million [11]

Also, shown in Figure 13 are the results for the double effectors located at the $70 \%$ and $86 \%$ upper surface location. Similar to the single effectors, the double effectors had shown signs of positive increase in lift coefficient passed the baseline stall point.

A look at the pressure distribution for the single effectors, Figure 14 (please see next page) further reveals the increase in lift seen is largely due to the increase in static pressure area near the leading edge of the airfoil section just before the presence of the effectors geometry on the upper surface. 


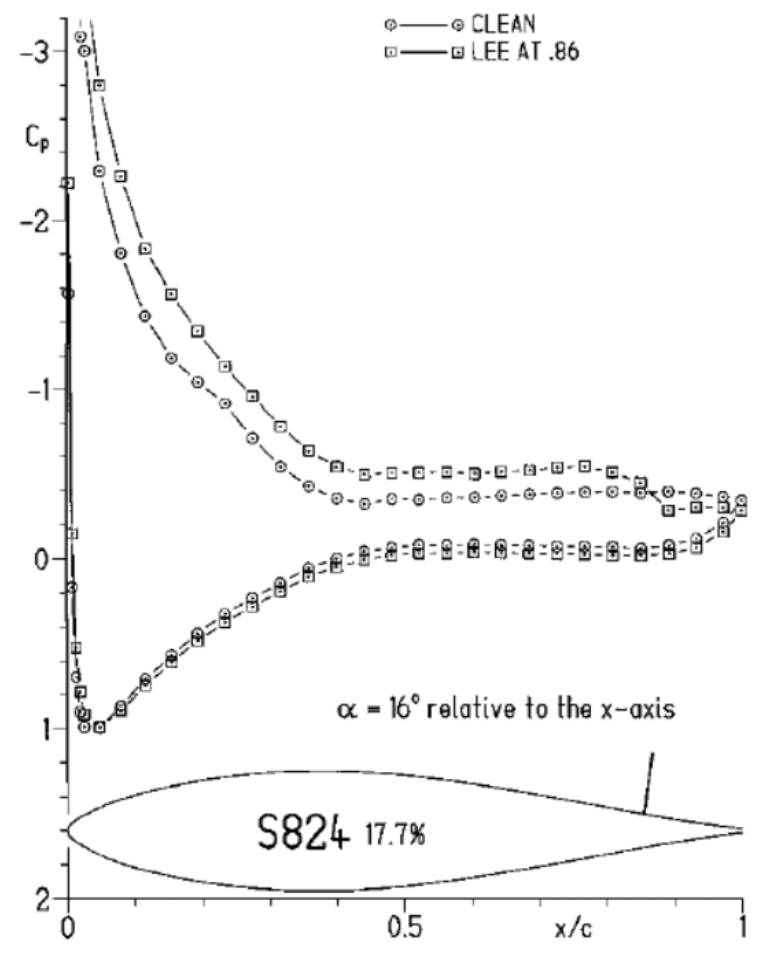

Figure 14: Pressure Distribution S824, 0.86\% chord location, Alpha $=16, R e=1$ million [11]

Therefore, the results of this study along with the previously mentioned articles, motivated this study to analyze the ability of a spoiler to function as a lift enhancing device that can be used as mechanism to effectively delay stall. Numerical performance of a rigidly attached spoiler-airfoil combination using Computation Fluid Dynamics was the prime focus for investigation. The thesis purpose was to further add to this line of research, using a numerical approach, on the use of spoilers as an alternate device to alleviate stall effects. The following chapters provide details on the methodology utilized for grid generation for both the baseline clean NACA2412 section and spoiler section. Grid independent study was carried out using Richardson's extrapolation for grid validation purposes followed by the main study of spoiler lift performance at high pitch angle of attack. 


\section{Chapter 2}

\section{Methodology}

The objective of the methodology study was the development of a numerical mesh that would serve as a basis for both the baseline and spoiler study. The goal of the study was to achieve mesh grids that were consistent in nodal and spatial layout. This was mainly carried out for the purpose of extracting useful comparisons between the two grids (baseline and spoiler). The grids were generated with the understanding that the distance of first node off the wall surface was targeted to produce a Wall $\mathrm{y}+$ of at least 1 (additional details provided in the next section of the report) as well as allocating enough nodes to the mesh for the purpose of reducing any error associated with the mesh constructed. The following section provides information on the methodology employed in generating the numerical grids that form the basis for this research.

\subsection{Baseline Mesh Information}

Airfoil sections are typically identified by the following parameters:

(i) The chord line which is defined to be the line connecting the leading and trailing edge points of an airfoil section.

(ii) The camber line which is an imaginary line that represents the midsection between the upper and lower surfaces of an airfoil section

(iii) The camber which is the maximum distance between the camber line and the chord line, given in terms of the chord line

(iv) The chord length which is the length of the chord line

(v) The Maximum thickness which is defined as the distance between the upper and lower curve sections of the airfoil, given in terms of percent chord length.

For ease of reference these parameter are shown in Figure 15: 


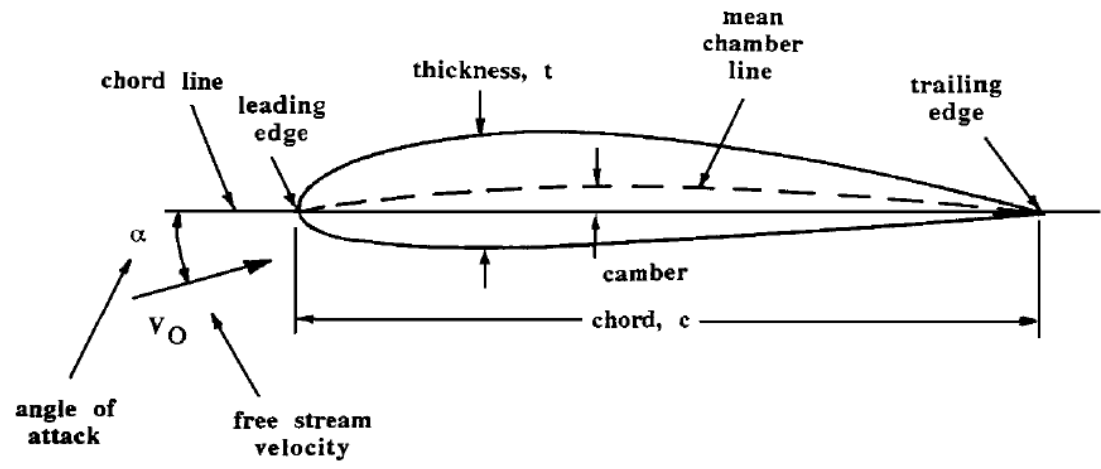

Figure 15: Airfoil parameter convention [12]

The NACA 2412 airfoil section, has a maximum thickness of $12 \%$, with $2 \%$ camber located at $40 \%$ maximum aerodynamic location. This airfoil section was chosen for its high demand in aircraft design. It is one of the most studied NACA section that has elements that similar to many commonly used wing profiles in terms of camber and thickness. It was also chosen for its clean trailing edge characteristics.

Before the advent of computational tools, extensive wind tunnel tests were conducted as means of understanding aerodynamics. A report compiled by Rogers et al. [13] completed an in-depth analysis of the flow characteristics for this airfoil section at low speeds, at a Mach of 0.13 and chord length of one meter corresponding to a Reynolds number of 2.2 million. The study was conducted for the purposes of shedding more light on flow separation which would aid researchers in producing high fidelity mathematical models in capturing such physics. These experiments were carried out at Wichita University in which two NASA airfoils sections, NASA LS(1)-0421, NASA GA(W)-2 were tested against the NACA 2412 airfoil section. Tests were conducted at three $\alpha$ values of interest: pre-stall 12.4 degrees, near stall 14.4 degrees and post stall 16.4 degrees. The following were the streamlines found for the flow separation shown in Figure 16: [13] 


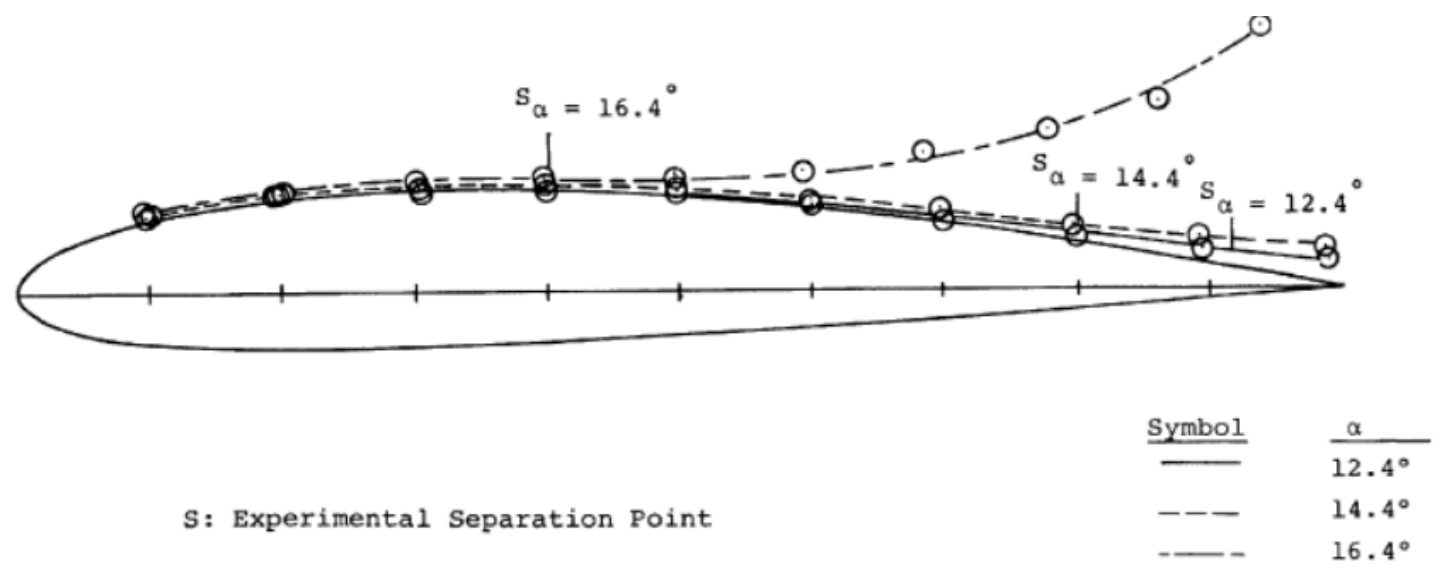

Figure 16: Streamlines for pre-stall, near-stall and post stall $\alpha$ values for NACA2412 at Reynolds Number of 2.2 million, M=0.13 [13]

As seen in Figure 16, the NACA 2412 exhibits well defined trailing edge stall behaviour, which made it the ideal choice for the conduction of this research.

The first step of the mesh study was to create the NACA 2412 airfoil geometry and using ANSYS ICEM CFD as the grid generator, produce a c-type topology for the far-field mesh distribution as the flow-field domain surrounding the NACA 2412 airfoil section. The imported coordinates representing the geometry of the NACA 2412 airfoil section were created using Abbott and Doenhoff's method of combing mean lines and thickness distribution. Without delving into the details, information on coordinate point generation for the NACA 2412 section can be found in the book, Theory of Wing Sections by Abbott and Doenhoff, Chapter 6; Families of Wing Sections.

Using 2D planar hexa meshing, and ANSYS ICEM CFD blocking technique the following subsections provides a detail look at the baseline mesh construction. 


\subsubsection{Baseline Blocking \& Nodal Mesh Details}

The construction of the mesh profile involved the generation of three main geometrical sections: Table 1: Mesh breakdown

\begin{tabular}{|ccc|}
\hline $\begin{array}{c}\text { Parameter Name } \\
\text { Domain }\end{array}$ & Description & Flow Field Boundary Condition \\
\hline Fluid & Mesh $(20 \times 30 \mathrm{~m})$ & Pressure Far field Distribution \\
\hline NACA 2412 Airfoil Geometry & Upper and Lower curves. & Fluid \\
& Total chord length of & Walls \\
& $1.008304[\mathrm{~m}]$ & \\
\hline
\end{tabular}

The total chord length stated in Table 1 was an extrapolated value from that originally suggested chord length suggested by Abbott and Doenhoff as 1 [m] [14]. This value was determined for the purposes of obtaining a sharp trailing edge, as from the import of the coordinate data points for the airfoil section (provided by Abbott and Doenhoff) had created a slightly blunt trailing edge. For more information on mesh details for the baseline study, please refer to Appendix A.

\subsection{Spoiler-airfoil Geometry and Mesh Details}

The following is the methodology used to construct the spoiler-mesh configuration.

\subsubsection{Spoiler Geometry}

The spoiler size was conservatively chosen to be approximately 0.10 [m] (10\% chord) [15]. Three upper surface locations were studied; 60\%, 65\% and 70\% leading edge locations. These upper surface locations used for investigation are very closely located to where the rear spar is located in commercial aircraft [15]. Spoiler geometry creation involved using points and merging curves to the NACA 2412 airfoil geometry. Spoiler base and hinge point connections (shown in Figure 17) were determined from the set of points defining the NACA 2412 upper surface curvature as demonstrated in the Figure 17. 


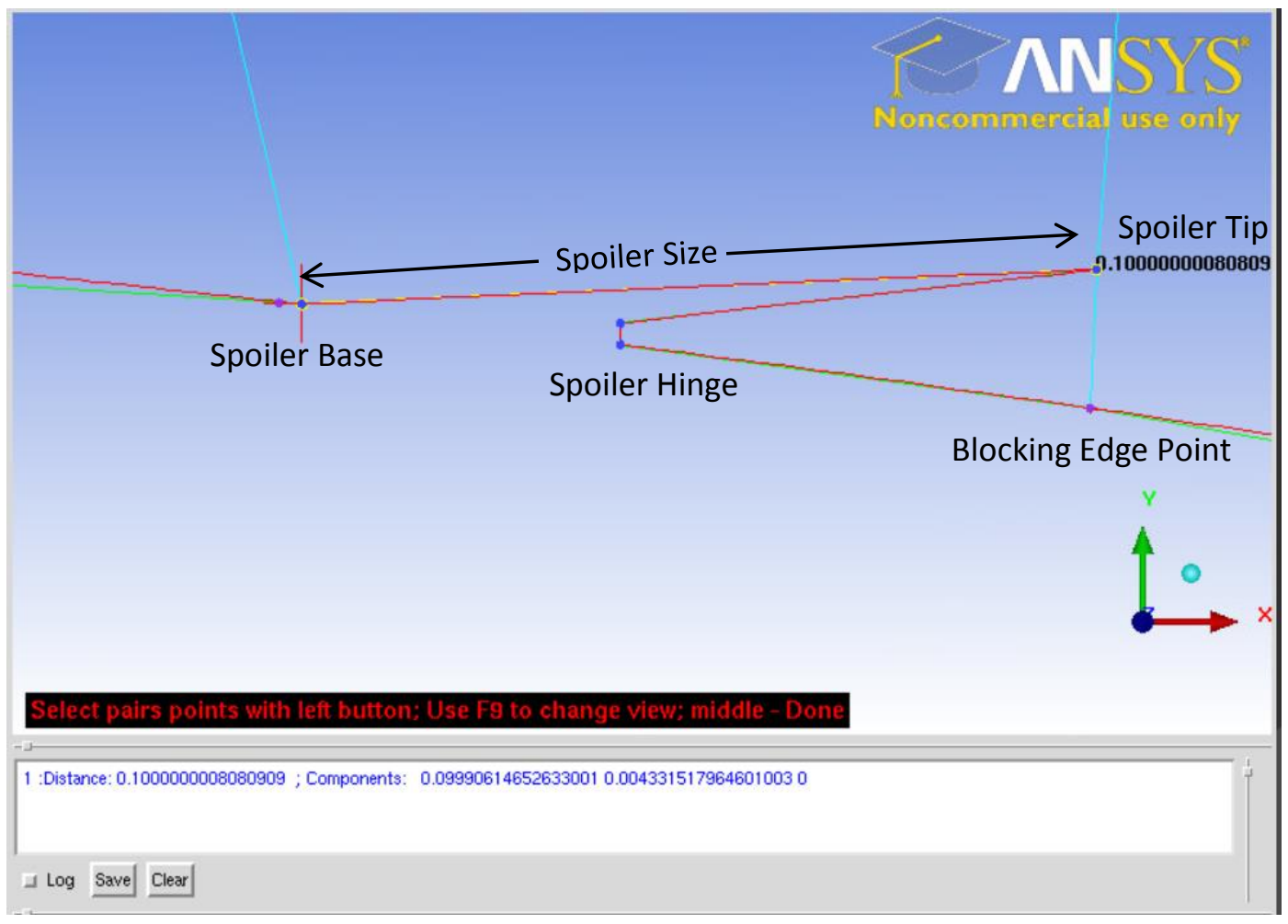

Figure 17: Spoiler Geometry Overview

The Spoiler Base in Figure 17 represents the leading edge connection points studied for this report. The Spoiler Hinge was given an approximate distance of $4 \%$ chord, along with a 5\% chord horizontal distance variation between the Spoiler base and Hinge connection points. The following table provides a summary of the points utilized to construct the spoiler geometry at the three locations of interests $(60,65$ and $70 \%$ leading edge upper surface locations): 
Table 2: Spoiler Coordinate Mesh Information

\begin{tabular}{|c|c|c|}
\hline Upper Surface Location & Parameter & $\begin{array}{l}\text { Cartesian Coordinate }(\mathbf{x}, \mathbf{y}, \mathbf{z}) \\
{[\mathrm{m}]}\end{array}$ \\
\hline \multicolumn{3}{|l|}{$60 \%$ chord, Leading Edge } \\
\hline & Spoiler Base & $(0.60,0.063,0)$ \\
\hline & Spoiler Hinge-Upper Point & $(0.64,0.0591,0)$ \\
\hline & Spoiler Hinge-Lower Point & $(0.64,0.0617,0)$ \\
\hline \multicolumn{3}{|l|}{$65 \%$ chord, Leading Edge } \\
\hline & Spoiler Base & $(0.65,0.055,0)$ \\
\hline & Spoiler Hinge-Upper Point & $(0.69,0.053,0)$ \\
\hline & Spoiler Hinge-Lower Point & $(0.69,0.055,0)$ \\
\hline \multicolumn{3}{|l|}{ 70\% chord, Leading Edge } \\
\hline & Spoiler Base & $(0.70,0.0518,0)$ \\
\hline & Spoiler Hinge-Upper Point & $(0.74,0.0464,0)$ \\
\hline & Spoiler Hinge-Lower Point & $(0.74,0.049,0)$ \\
\hline
\end{tabular}

Changes in spoiler deflections at a particular location were induced by varying the spoiler tip in a counter clockwise direction, while maintaining fixed spoiler base, and hinge point connections. The angle of deflection was created by rotation of the spoiler tip about the spoiler base point; taking the NACA2412 curvature as the reference zero horizontal. An example of a 10 degree offset in spoiler deflection is shown below. 


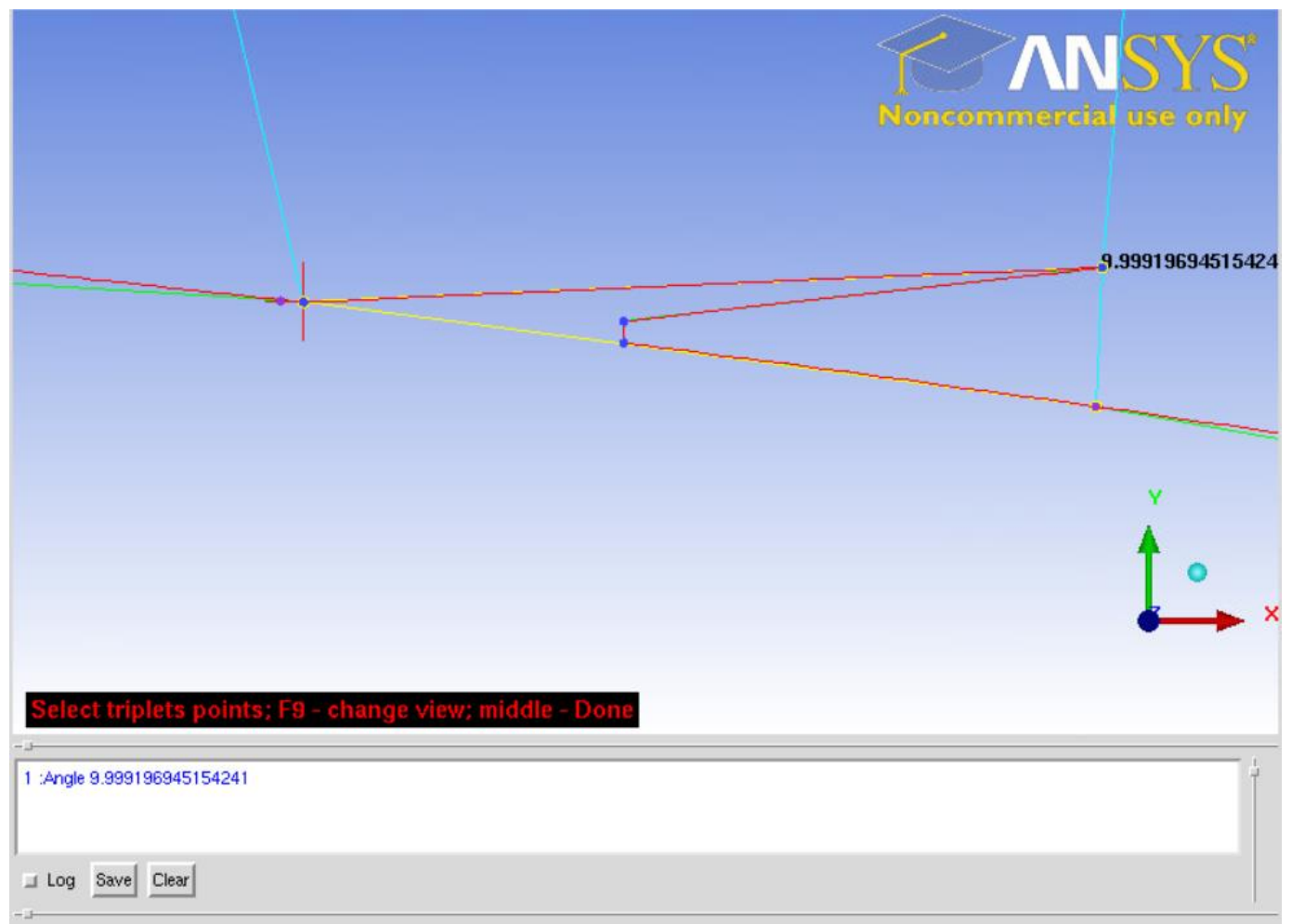

Figure 18: NACA 2412 airfoil-Spoiler deflection overview

\subsubsection{Spoiler Blocking \& Nodal Mesh Overview}

Blocking was carried out using a $\mathrm{C}$ mesh topology for the far field distribution similar to what was configured for the baseline case. The blocked area under the spoiler tip region was closed off for two primary reasons: ease of meshing, and little influence in the solution due to slow moving air in that area. For spoiler blocking and nodal mesh details, please see Appendix B. 


\section{Chapter 3}

\section{Baseline Study}

\subsection{Experimental Study}

The objective of the base-line study was to create a data set to serve as a baseline to be able to compare and substantiate spoiler influence in mitigating stall effects at high alpha. The experimental data collected by Abbott and Doenhoff corresponding to a Reynolds number of 3.0E6 for the NACA 2412 airfoil section was selected as the baseline flow characteristic in which values for lift, drag and moment coefficient (quarter chord) were readily available. [14] The published experimental values of these authors are commonly used for validation in aerodynamic studies.

According to Abbott et al. [14] the flow conditions in which the test data was obtained was produced in a low speed wind tunnel that had a pressurized capacity of up to 10 atmospheres. The tunnel itself had a test section measured to be 3 feet wide by 7.5 feet in height. The NACA models were approximately 2 feet in chord length and were said to span the full 3 feet wide test section to avoid any end wall effects. Data collected for the determination of lift and drag

coefficients involved integrating the pressure profiles measured between the floor and ceiling of the test section. Wake velocity profiles were used to compute the drag. The moment coefficient about the quarter chord was collected using balance equipment. More importantly, the maximum Mach number was set at 0.17 between the Reynold's Number range of 3-9 million [14]. Therefore, using this information, the following are a set of assumptions that were used to determine reference parameters required for fluid simulation in ANSYS Fluent (Version 14.0/14.5): 
Table 3: Baseline Flow Assumptions

\begin{tabular}{|cc|}
\hline Parameter & Value \\
\hline Density- Ideal gas & $1.176674 \mathrm{~kg} / \mathrm{m}^{3}$ \\
\hline Temperature- Standard & $300 \mathrm{~K}$ \\
\hline Air Pressure & $101 \mathrm{kPa}$ \\
\hline Altitude & Sea-level \\
\hline Mach & 0.17 \\
\hline
\end{tabular}

Sutherland's Constant Equation was utilized to accurately determine the variations of viscosity due to using the standard base temperature of $300 \mathrm{~K}$. Therefore, applying the initial assumptions stated in, flow characteristics for the speed of sound, flow speed, viscosity and ultimately the Reynolds Number were computed as the following:

$$
\begin{gathered}
\text { Speed of sound: } \boldsymbol{a}=\sqrt{\boldsymbol{\gamma R T}}=\sqrt{1.4 * 287 * 300}=347\left[\frac{\mathrm{m}}{\mathrm{s}}\right] \\
V=M a=0.17\left(347\left[\frac{\mathrm{m}}{\mathrm{s}}\right]\right)=58.99\left[\frac{\mathrm{m}}{\mathrm{s}}\right]
\end{gathered}
$$

$$
\text { Sutherland's Constant: } \boldsymbol{\mu}=\mu_{0}\left(\frac{T_{0}+S}{T+S}\right)\left(\frac{T}{T_{0}}\right)^{3 / 2}=1.8461 E-05
$$

$$
\text { Reynolds Number: } \operatorname{Re}=\frac{\rho V c}{\mu}=3.79 E 6
$$

Therefore, applying the above conditions in the fluid domain settings of the Transition SST turbulence model values of the lift, drag and moment at the quarter chord position were collected at various $\alpha$ values between the range of 4-20 degrees. The steady state solver was utilized for low $\alpha$ values in which the courant number was steadily varied accordingly to achieve a steady state solution. For the case of high $\alpha$, the transition SST solver was utilized as a conservative choice to provide the most effective solution to the unsteady physics seen for $\alpha$ past the stall point. A time step of 1E-05 was used in the transient solver to determine the aerodynamic characteristics (lift, drag, and moment about the quarter-chord), per iteration. This time step value was a conservative estimate computed as a ratio between the size of the smallest cell wall in the direction of the fluid flow and the free stream fluid velocity [16]. Re-visiting the 
methodology section of this report reveals for the airfoil-spoiler geometry case the smallest cell length in the direction of flow (taken to be the x-direction) was approximately determined to be $0.003[\mathrm{~m}]$ and the free stream velocity was $59[\mathrm{~m} / \mathrm{s}]$. For the transient cases, the initial start-up involved providing the transient solver a steady state initial condition in which the target was for the residuals to drop 3-4 magnitudes below their initial start. Therefore, the courant number was set to a maximum of 10 and the solution was allowed to iterate until the residuals were seen to fall below the target value. Once this was achieved, the transient solver was set to iterate and march in time with 5-10 iterations between each time step. An implicit scheme in which the second order up-winding algorithm for the fluid flow was utilized for both the steady state and transient solver. Convergence for the steady state solver was achieved through point monitoring of both the residuals (reduction in normalized value of at least an order of 1e-03 or less) and achieving the point in the simulation in which the lift and drag coefficient were seen to change by negligible amounts over time. The transient solver simulation was initiated using the solution of a steady state simulation which had been converged by an order of magnitude of 1e-03 or less. Results for the transient cases, of lift and drag coefficient, were determined by an averaging process. The assumption that the transient solver would produce comparable results for the solutions of low $\alpha$ provided by the steady state solver was assumed. This assumption was based on the understanding that flow separation is not a major determinant to produce unsteady physics for the cases of low $\alpha$ and therefore, using either the transient or the steady state solver would produce comparable results in value. For this study the lift coefficient versus angle of attack is shown in Figure 19. A plot of the drag coefficient and pitching moment versus angle of attack are in Figure 20 and Figure 21 respectively. 


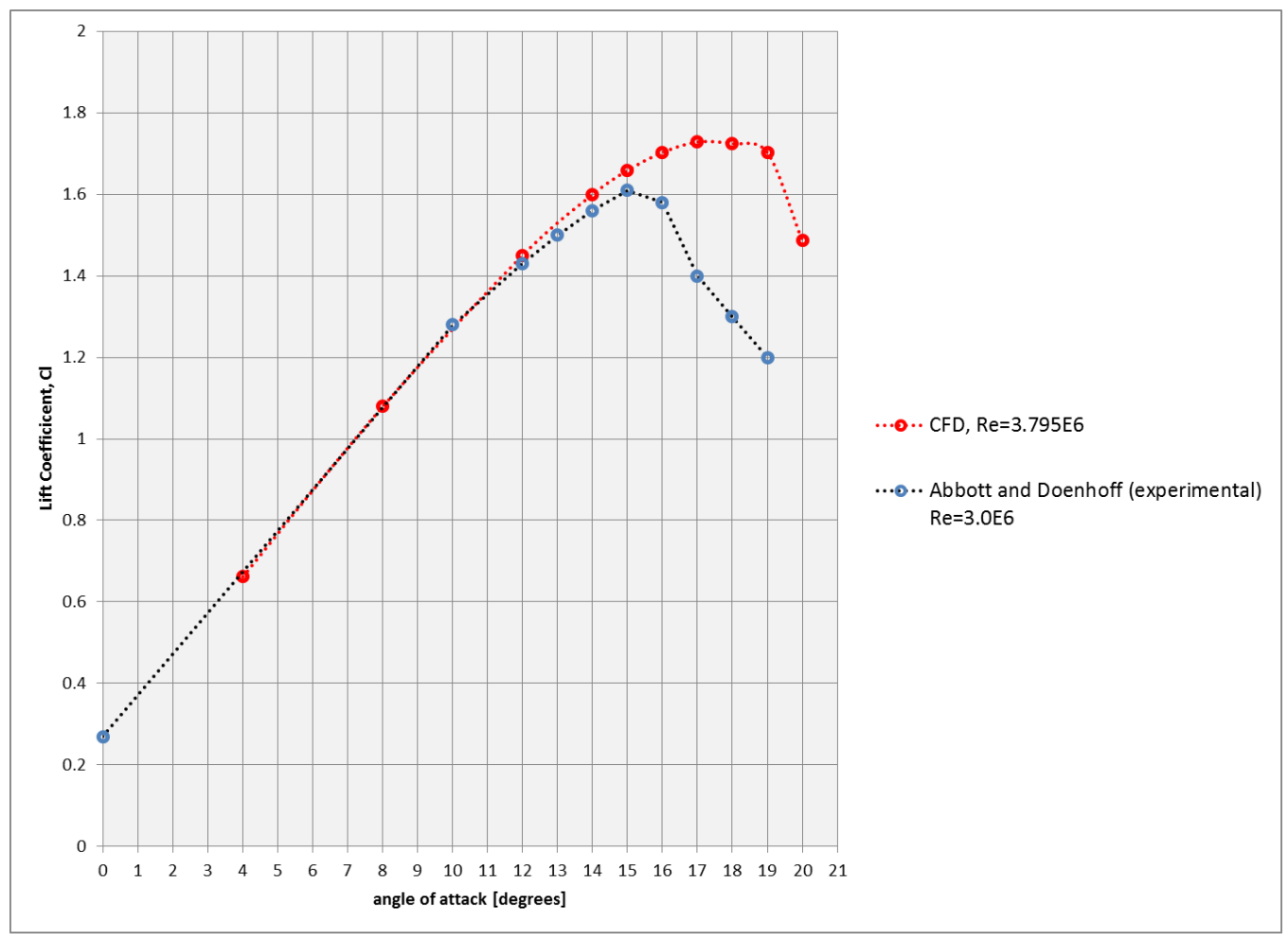

Figure 19: Baseline Study, Lift Coefficient, Transition SST Model

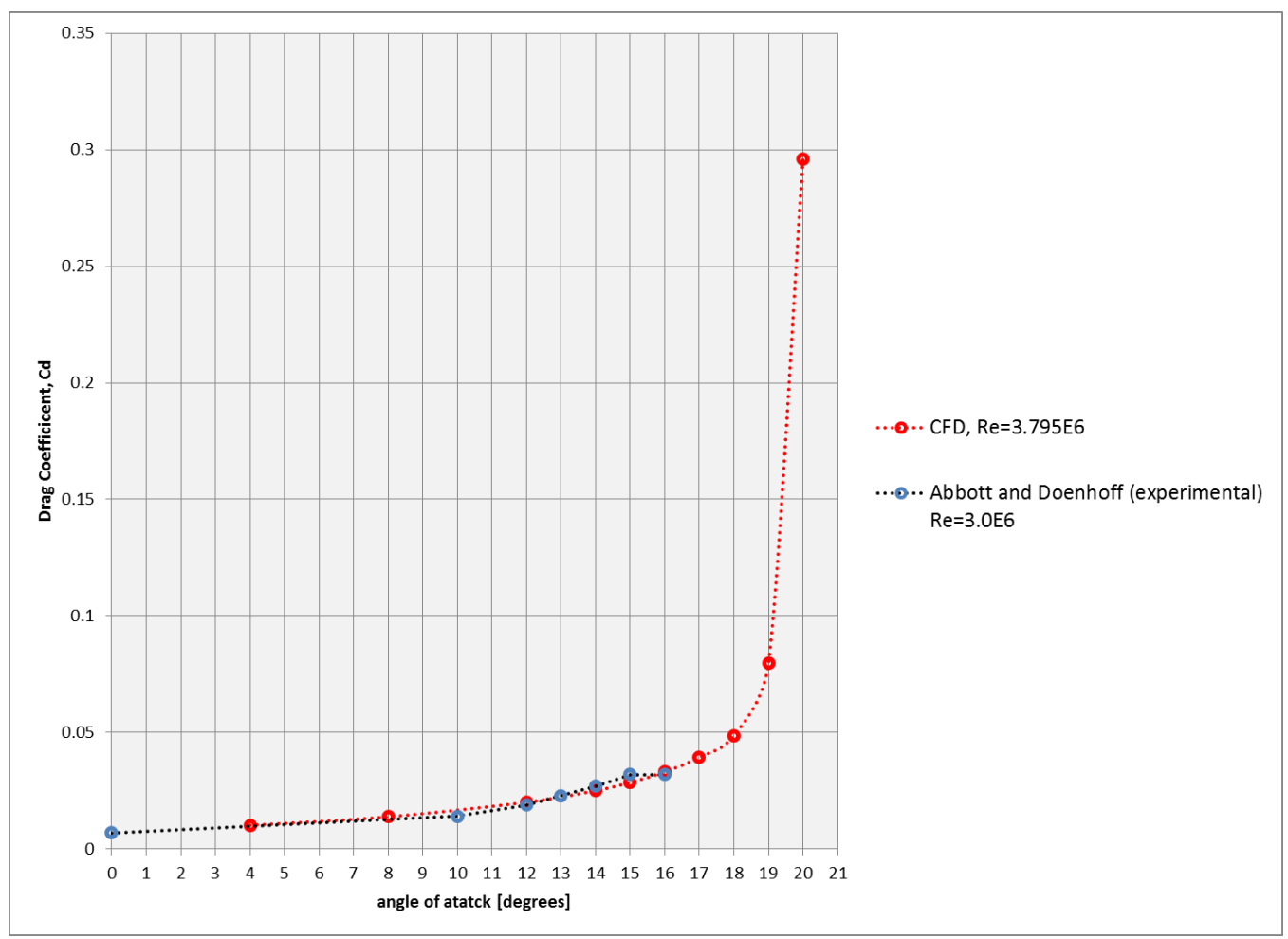

Figure 20: Baseline Study, Drag Coefficient, Transition SST Model 


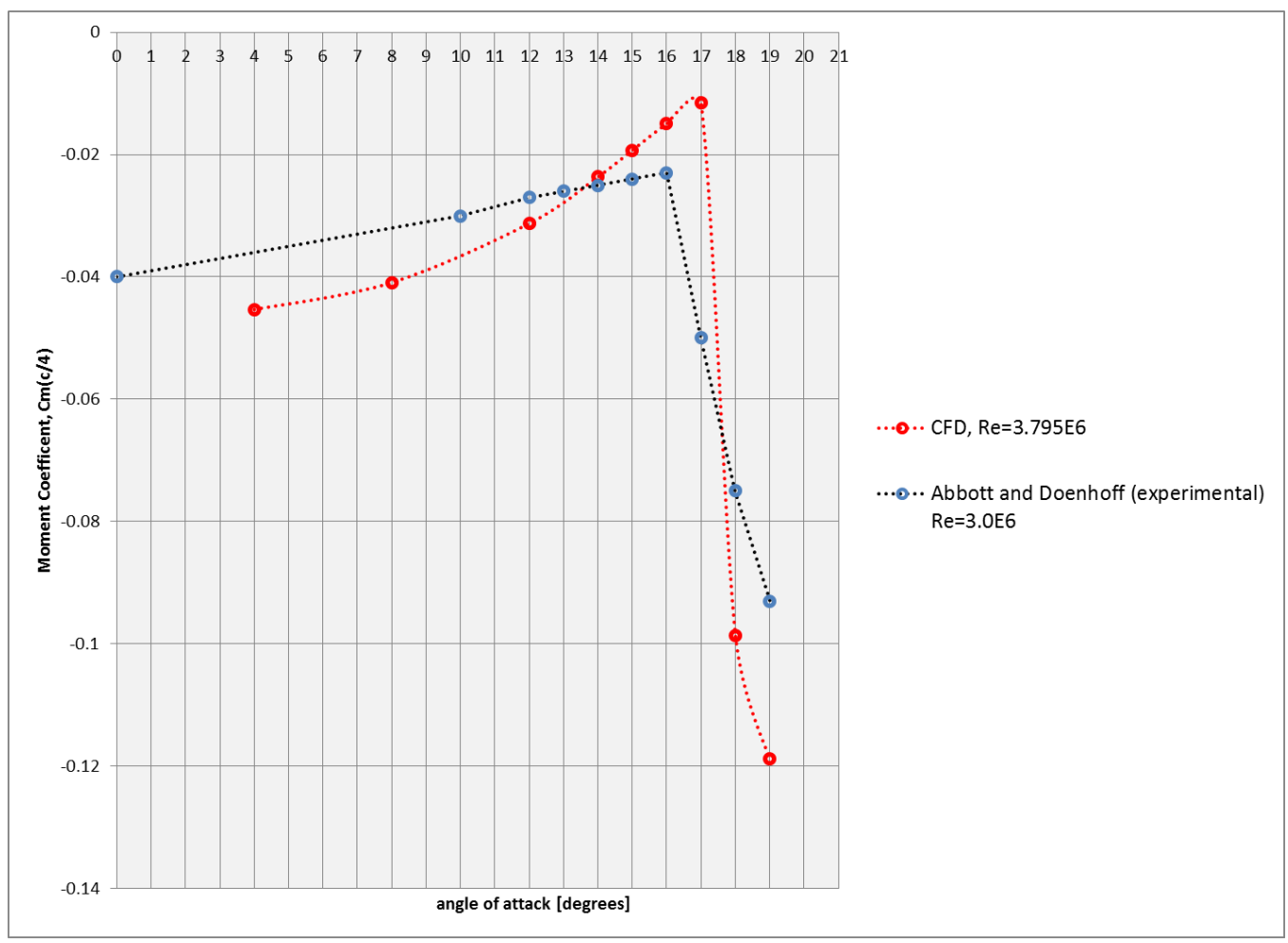

Figure 21: Baseline Study, Moment Coefficient at Quatre Chord, Transition SST Model

As can be seen in Figure 19-Figure 21, the Transition SST model provides fairly reasonable results for the lift and drag coefficient prior to an alpha of 12 degrees in comparison to that recorded experimentally by Abbott and Doenhoff. Numerical results for the lift coefficient past 12 degrees were seen to be slightly higher in value. Note, that the experimental values for Abbott and Doenhoff in these plots were estimated from there published plot. Nonetheless, the Transition SST model provides fairly reasonable values for the lift and drag coefficient, but a more thorough investigation into model selection is provided in the following chapter. The moment coefficient at the quarter chord point was estimated to have a level of accuracy of one significant figure making it more difficult to accurately resolve. Nonetheless, for low values of $\alpha$ the numerical trendline was seen to be fairly close to the experimental. Overall, in consideration of the primary objective of this study, it was concluded that the initial assumptions of standard temperature, and pressure, at a Mach of 0.17, using Sutherland's estimation for Viscosity provide adequate results that may serve as the baseline case. 


\subsection{Model Selection}

The central focus of this research involved effectively capturing the separation bubble developed due to the adverse pressure gradient present at high alpha, near the upper trailing edge surface of the NACA 2412 airfoil section. Coupled with the turbulent nature of the flow, an unsteady Number; Reynolds Averaged Navier-Stokes (URANS) coupled with a turbulence model was chosen as the solver for investigation. The critical criteria for a turbulence model selection was model sensitivity to near wall effects. The objective was to determine the turbulence model that most optimally resolved the separation bubble, in addition to key aerodynamic forces for the lift and drag coefficients for a select range of values in alpha.

Boundary layer turbulence is essentially a product of viscous effects near solid wall surfaces, which in this particular case would be airflow near the upper/lower surfaces of the airfoil section. Viscosity plays a significant role in drag via frictional forces. Velocity of the flow increases to a value near the farfield a short distance away from the wall, leading to a large velocity gradient at the wall.

Boundary layer turbulence is thus created by the strong velocity gradients near the wall and in numerical computational fluid dynamics the model's capability of resolving such a feature is highly dependent on approximations of the momentum transport equations.

In the simplest form, fluid physics is dependent on the three governing laws:

1) Conservation of Mass (Continuity Equation) mass entering a control volume must be equal to the mass exit out of the control volume

2) Momentum equations: Derived from Newton's second law, which states that the rate of change of momentum equals the applied force

3) Conservation of Energy via the First law of Thermodynamics: Energy cannot be created or destroyed but can be transferred from one form to another.

Focusing specifically on momentum, shear stress originates from the surface forces present on the control volume under investigation. For more information on shear stress please see Appendix D of this report. 
Four turbulence models were investigated:

(i) The one-equation Spalart-Allmaras model

(ii) The two-equation $\mathrm{k}-\omega$ Transitional model

(iii) The two-equation k-epsilon Model

(iv) The four-equation Transition SST Model

The methodology involved utilization of the baseline mesh as input into the models and alpha values of $4,8,12,14,16-18$ degrees. ANSYS Fluent 14.0/14.5 was used as the software of choice and important features of the domain that remained unchanged throughout the test involved the following settings:

Table 4: Parameter Settings in ANSYS Fluent

\begin{tabular}{|cc|}
\hline Parameter & Setting \\
\hline Fluid, Air & Ideal Gas \\
\hline Viscosity & Sutherland's Law \\
\hline Mach & 0.17 \\
\hline Temperature & $300 \mathrm{~K}$ \\
\hline Pressure & $101 \mathrm{kPa}$ \\
\hline
\end{tabular}

Post analysis of each case tested with the turbulence models specified above involved examining contour plots for velocity, as well as wall shear stress, wall y+ and pressure coefficient plots.

Contour plots for velocity were utilized for the purpose of visually identifying the separation zone near the trailing edge. Areas of reduced flow, particularly near the wall regions, represented the separation zone. The overall shape of the separation zone provided information on the degree of severity of flow separation seen on the upper surface. In addition, the contour levels provide information on the various velocity gradients seen across the separation zone.

Plots for the wall shear stress along the upper and lower surfaces of the NACA 2412 airfoil section were extracted for the purpose of determining the location of the separation point. The 
separation point can be determined by the following relation provided by the wall shear stress distribution:

$$
\tau_{w}=\mu \frac{d U}{d y}
$$

Where du/dy is the velocity gradient at the wall in the normal direction.

The location at which the wall shear stress is found to equate to zero is the defining point of separation.

The wall $y+$ is the non-dimensional distance from the wall and is used by turbulence models. Wall $y+$ is defined to contain the characteristic length-scale displayed as the following:

$$
y^{+}=\frac{y \tau_{w}}{v_{T}}
$$

The velocity distribution is defined in the laminar sub layer to be:

$$
U^{+}=\frac{1}{\kappa} \ln y^{+}+5
$$

According to literature, a plot of the $y+, \mathrm{U}+$ distribution, commonly termed as the "law of the wall" is shown to be the following: 


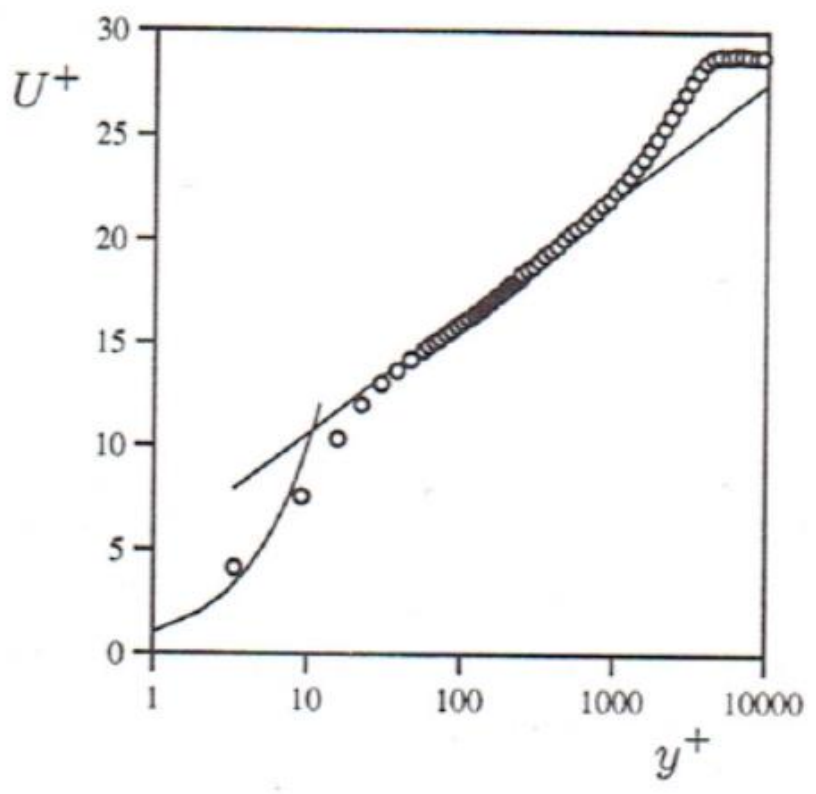

Figure 22: Mean velocity distribution $U+v s$. Wall $Y+[17]$

The area of interest in Figure 22 is in the region where the U+ distribution equals wall $y+$, which is in the linear region between 0 and $5 \mathrm{Y}+$. This region is known as the representing the pure viscous sub layer, whereas the buffer layer and overlap layer are defined to be in the following regions:

$$
\begin{gathered}
\text { buffer layer: } 5<y^{+}<70 \\
\text { overlap layer: } 70<y^{+}
\end{gathered}
$$

Therefore, for sufficient resolution of turbulent flow physics near the wall, the nodal distribution near the wall must produce a wall y+ distribution within the confines of that defined as the pure viscous sub layer. Conservatively, from past studies, the wall y plus distribution for turbulence modeling ideally should be around an order of one. [17]

Lastly, the two dimensional pressure coefficient plots provide information on the overall lift production across the spanwise location of the airfoil. An overlay of the pressure coefficient plots provides details on the regions of the airfoil that contribute to a greater degree to the lift coefficient seen. In addition, surface integration of the pressure distribution provides the lift and a portion of the drag force values calculated for a particular angle of attack under considerations. The pressure coefficient is defined to be the following: 


$$
C_{p}=\frac{p-p_{\infty}}{\frac{1}{2} \rho_{\infty} V^{2}}
$$

The results for the model study using the lift and drag coefficient across the turbulence models tested can be found in the following plots. Note that NACA 2412 results corresponding to a Reynold's number of 3.0E6 collected by Abbott \& Doenhoff were overlaid with the results for comparison purposes.

As can be seen in Figure 23, shown on the following page, the lift coefficient for $\alpha$ values of 4, 8, 12, 16-18 for each turbulence model tested, followed an overall smooth transition in the trend line between alpha values tested.

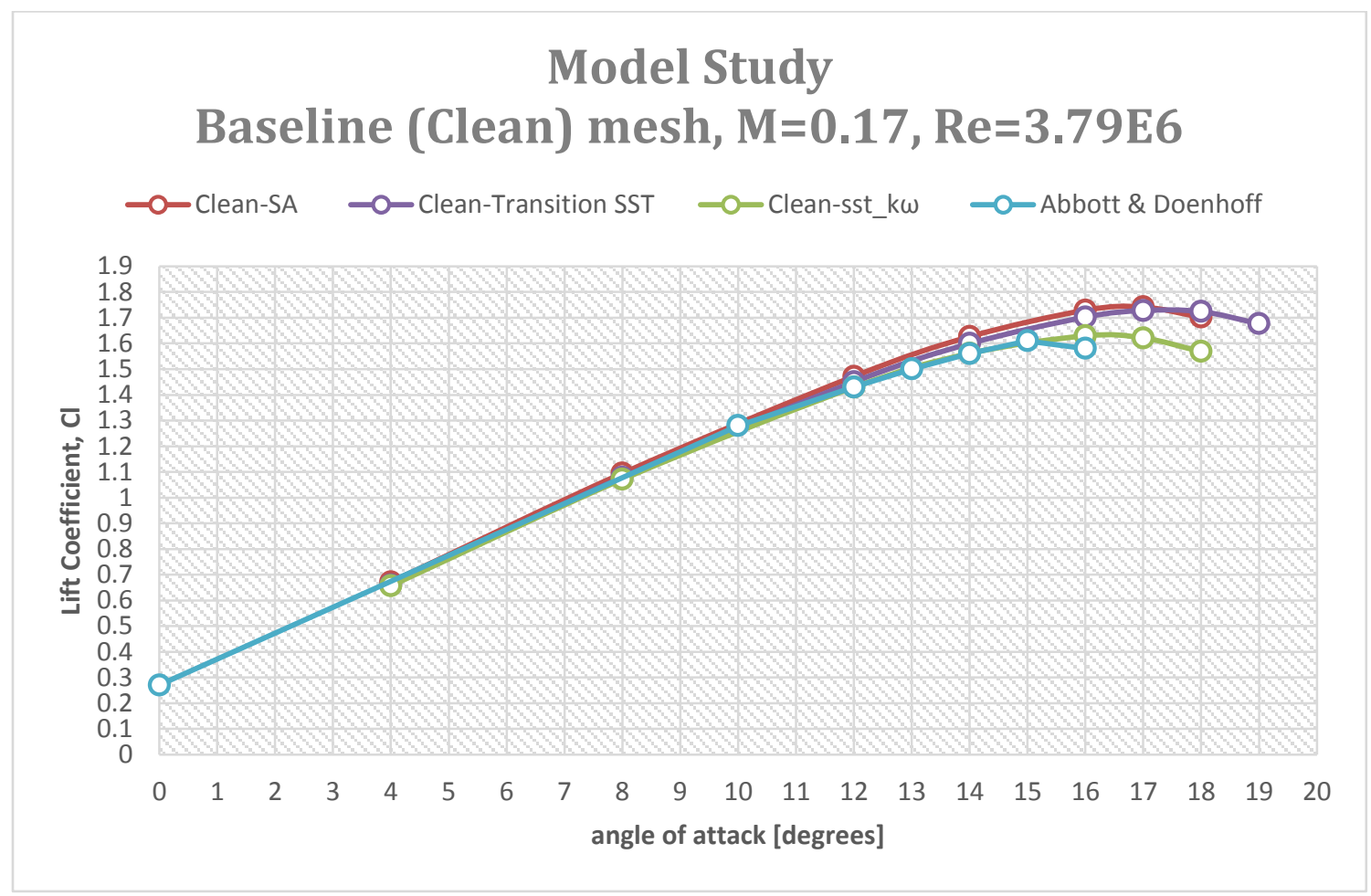

Figure 23: Lift-alpha Plot for Model Study of NACA2412 airfoil Clean Mesh, M=0.17, Re=3.79E6 


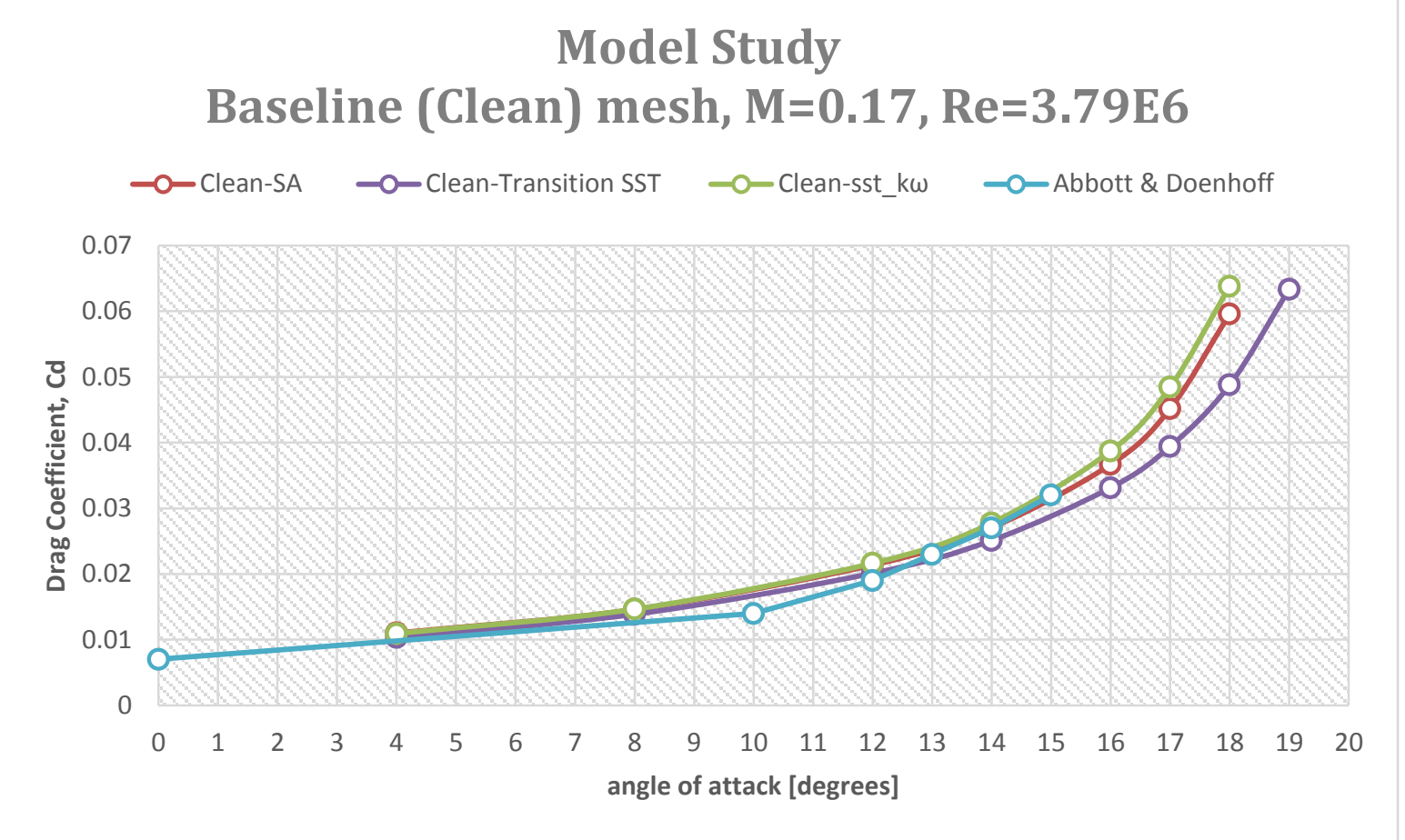

Close examination of the lift-alpha curve in Figure 23 reveals that the maximum lift coefficient

Figure 24: Drag- alpha plot for Model Study of NACA2412 airfoil Clean Mesh, M=0.17, Re=3.79E6

was found to be produced by the Transition SST model with an approximate value of 1.728 for a maximum alpha value of 17 degrees. Comparatively, Abbott and Doenhoff experimental results, had shown a maximum stall angle of 15 degrees with a lift coefficient of 1.6. Lift coefficient values found for lower $\alpha$ of 4 and 8 degrees show almost identical values in output across all the turbulence models tested. Intuitively these results are expected as there is very little boundary layer separation found at these low angle of attack cases. This finding is further supported by the overall lift-alpha curve slope found to be almost equal in all turbulence models tested prior to an alpha of 12 degrees. Similarly, results for the drag coefficient at the low range $\alpha$ had also shown to be roughly equal in numerical output. This suggests that any of the turbulence models tested (transition SST, S-A, k-epsilon or SST k-omega) are valid models that can be utilized for values of $\alpha$ prior to 12 degrees.

Flow separation was detected past 12 degrees alpha, in which case both the S-A model and Transition SST model had shown close correlation in numerical output for values of the lift coefficients seen in Figure 23. The Transition SST model showed a slight dip in the lift 
coefficient from 17 to 18 degrees (therefore a small loss in lift was seen) in comparison to the SA model, thereby suggesting the Transition Model would be the ideal choice for this study. Accordingly, a look at the drag coefficient for the high $\alpha$ tested had shown a fairly large distinction in resolution between the Transition SST model and S-A model. The drag coefficient values particularly for high alpha values between the range 16-18 degrees had shown on average of at least a $10 \%$ difference in value detected for each alpha the between S-A and Transition SST model tested. For example, the drag coefficient values corresponding to the Turbulence models tested can be found in Table 5. Note that results for the SST-kw model were added for comparison purposes.

Table 5: Drag Coefficient Values for Clean NACA2412 Mesh, M=0.17, Re=3.79E6

\begin{tabular}{|cccc|}
\hline Alpha & Transition SST & S-A & SST-kw \\
\hline $\mathbf{1 6}$ & 0.033 & 0.0366 & 0.0381 \\
\hline $\mathbf{1 7}$ & 0.039 & 0.0451 & 0.0484 \\
\hline $\mathbf{1 8}$ & 0.048 & 0.0596 & 0.0637 \\
\hline
\end{tabular}

Therefore, preliminary results for aerodynamic characteristics of drag and lift coefficients for the clean mesh dictate that the Transition SST model would be a reasonable choice as the model produced the highest lift coefficient, at a stall angle further aft of that seen across all model tested, in addition to that suggested by Abbott and Doenhoff. Nonetheless, contour plots and results found near the wall were examined to further validate such a finding. The following are the model study results found for an alpha of 16 degrees. 


\section{Model Study: Wall Shear Stress Variation}

Baseline (Clean) mesh, $M=0.17, \operatorname{Re}=3.79 E 6, \alpha=16$ degrees

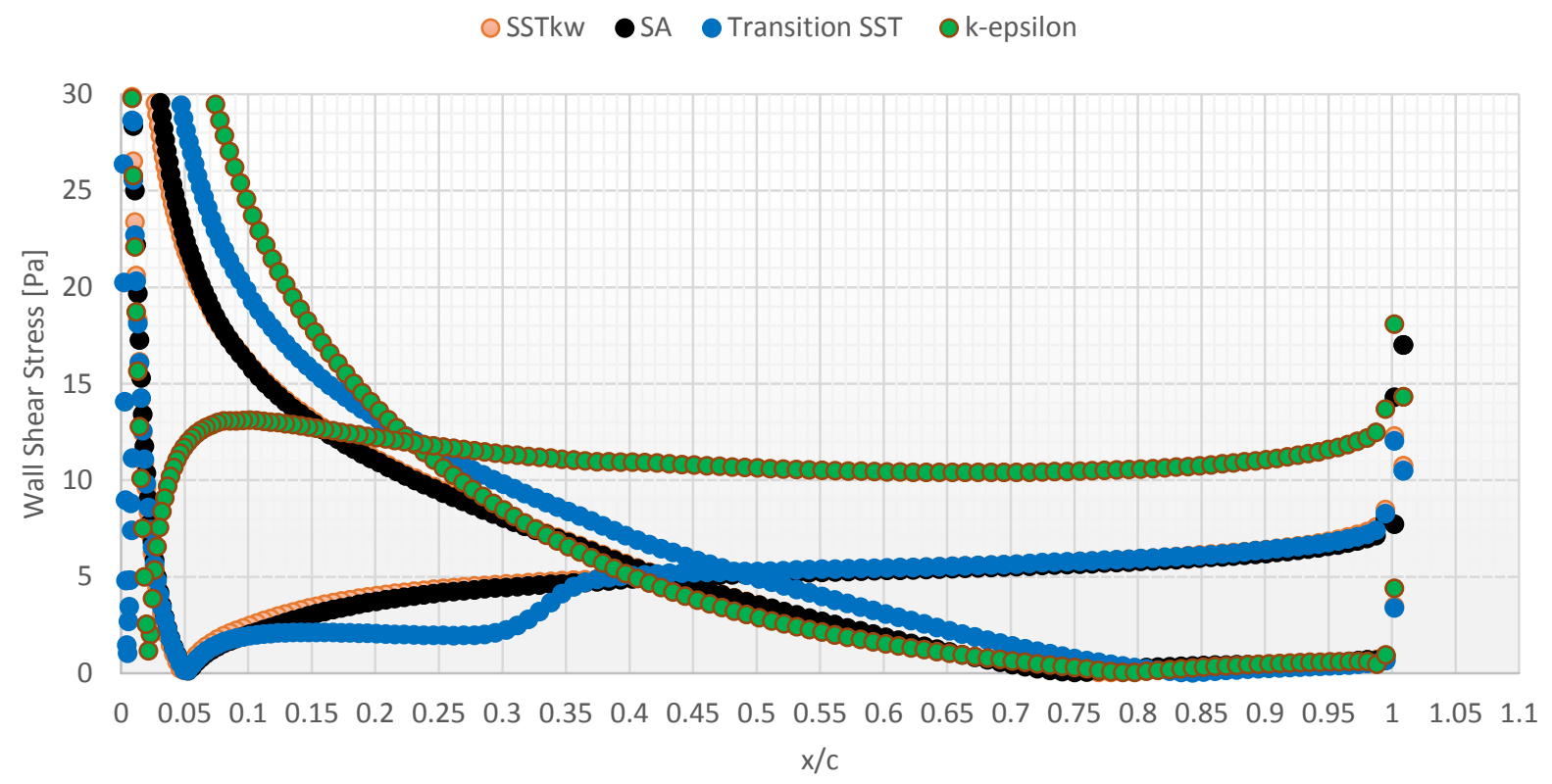

Figure 26: Model Study, Wall shear Stress Plots for Alpha=16, Clean Mesh, M=0.17, Re=3.798E6

\section{Model Study: Wall Shear Stress Variation} Baseline (Clean) mesh, $M=0.17, R e=3.79 E 6, \alpha=16$ degrees

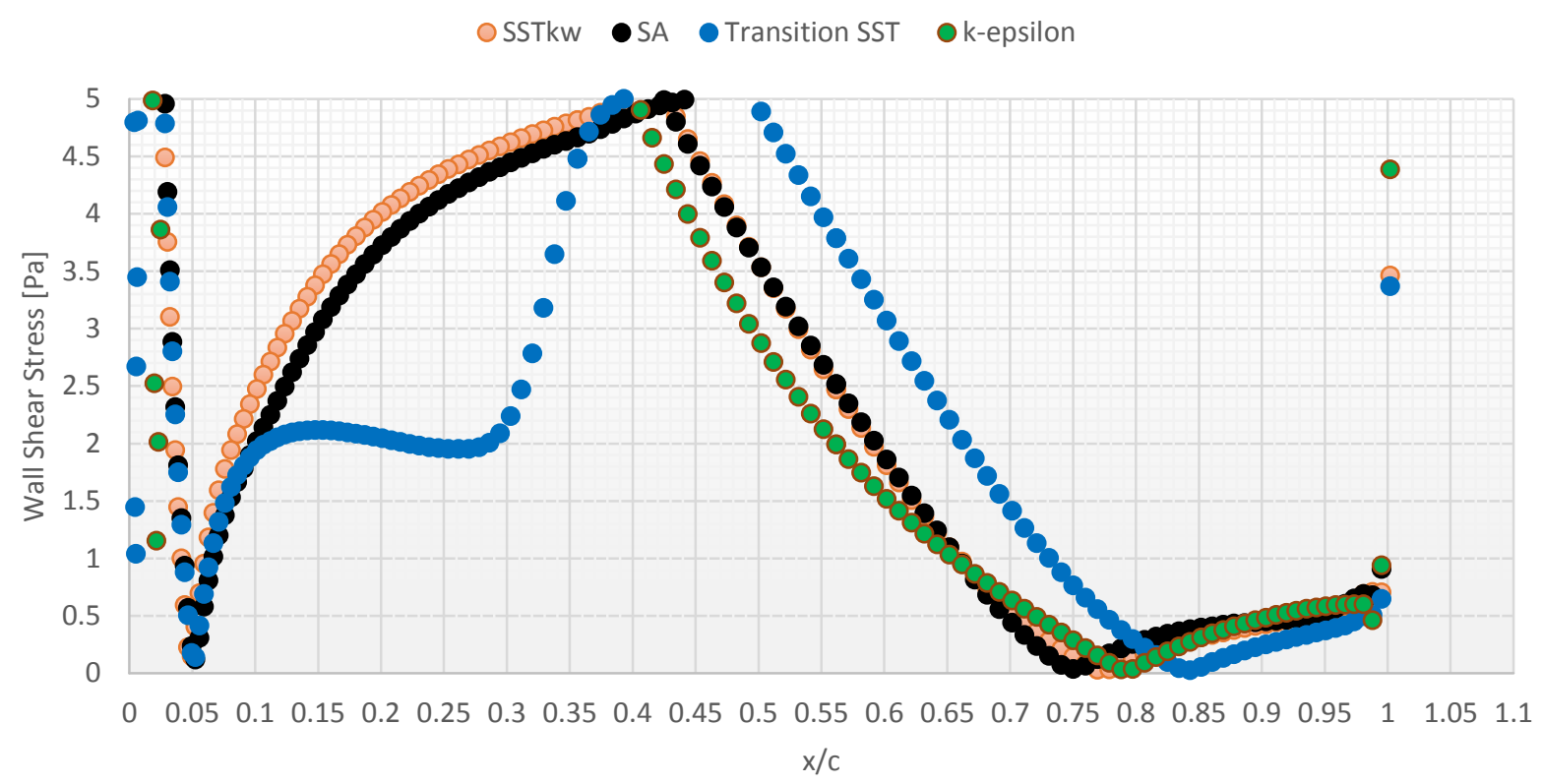

Figure 27: Truncated Wall Shear Stress Plot, Baseline Mesh, Alpha=16 (M=0.17, Re=3.79E6) 


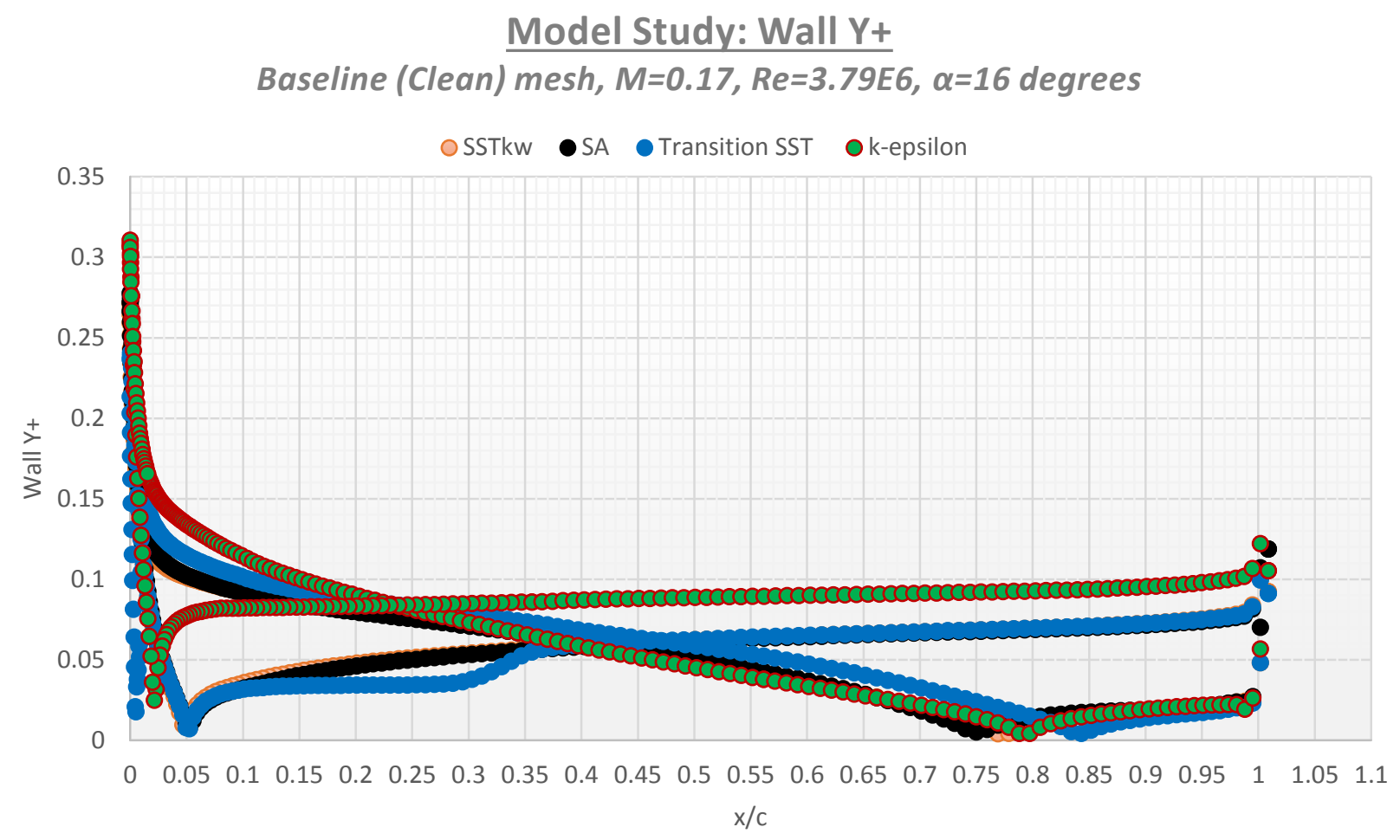

Figure 28: Wall $y+$ Plot for Alpha=16, Baseline Mesh $(M=0.17, \operatorname{Re}=3.79 E 6)$

\section{Model Study: Pressure Coefficient \\ Baseline (Clean) mesh, $M=0.17, \operatorname{Re}=3.79 E 6, \alpha=16$ degrees}

OSSTkw -SA - Transition SST Ok-epsilon

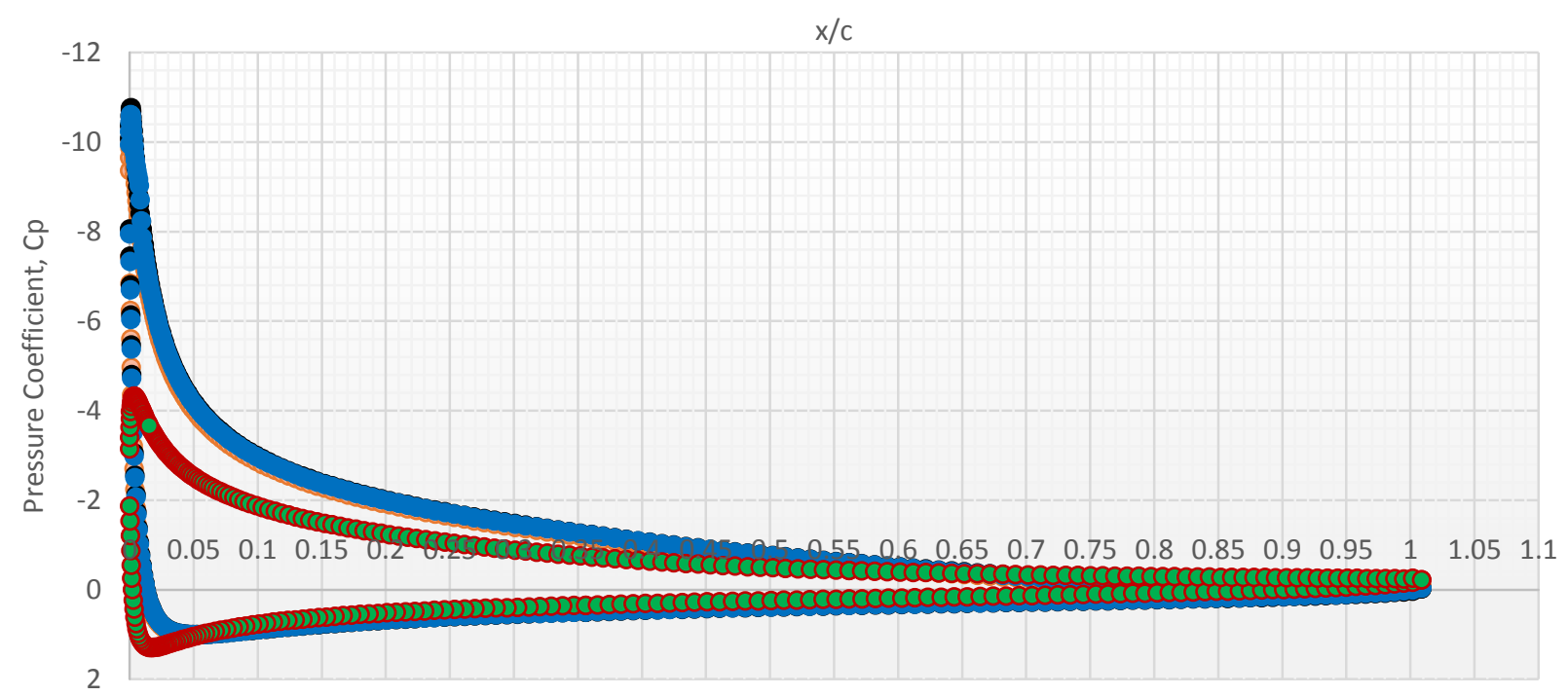

Figure 29: Pressure Coefficient Plot for Clean Mesh, Alpha=16 (Mach=0.17, Re=3.79E6) 
Post analysis of the results for an alpha of 16 degrees in Figure 25, show the separation zone on the upper surface of the NACA 2412 airfoil trailing edge section to be significantly smaller in area for the Transition SST model in comparison to the sstk $\omega$ and the ke model. This is visually apparent by analyzing the separation angle in which the flow deflects off the upper surface region. The deflection angle of the separated flow is shown to be farther upstream in the sstk $\omega$ model in comparison to that seen in the Transition SST model in Figure 25. In comparison, the $\mathrm{k} \varepsilon$ model produced the greatest area of flow separation shown by the low velocity contours displayed in Figure 25. Overall, it appears that the S-A model results are comparative to that seen for the Transition SST model, however the lift-alpha curve in Figure 23 shows a slight drop in lift past the stall point for the S-A model in comparison to that seen for the Transition SST model.

This observation was further validated through a look at the wall shear stress plots shown in Figure 26. According to the results, the S-A model, Transition SST and sstk $\omega$ models show overall similar trends for the wall shear stress on both the upper and lower airfoil surface walls, in comparison to ke model. Thereby suggesting that since both the velocity contours and the wall shear stress plots had demonstrated a trend favouring low lift production and high drag characteristics, the k-epsilon model was not the ideal choice. The results from the Transition SST model had shown a distinct transition in shear going from laminar to turbulent; none of the other model had shown this therefore, as this what is expected in the real world, the Transition SST model makes it the best fit choice for the Spoiler study. Note that the search for numerical results with a similar Reynold's Number from previously published papers was inconclusive; therefore the results for the model study within this section of the report are strictly compared to one another.

Close examination for the location of the separation point was determined through truncation of the wall shear stress plot to display points between 0-5[Pa] as shown in Figure 27. The focus, as mentioned earlier, was to determine the location along the upper surface that corresponds to a wall shear stress of zero. This plot clearly shows the separation point to be approximately $0.75 \mathrm{c}$ for the S-A model, $0.78 \mathrm{c}$ for sstk $\omega$, and $0.85 \mathrm{c}$ for the Transition SST model. Therefore, numerically the Transition SST model had favoured a shift of the separation point to 
approximately $10 \%$ further aft along the chord toward the trailing edge on the upper surface airfoil section.

Figure 28, shows the wall y+ distribution along the upper and lower airfoil surfaces for all models tested at an alpha value of 16 degrees. The plot displays a maximum wall $y+$ value of $\sim 0.3[\mathrm{~Pa}]$ at the leading edge; tapering off to below $\sim 0.15[\mathrm{~Pa}]$ along the duration of the upper and lower surfaces. Therefore, as it is mandatory for the wall $y+$ to be in the viscous sub layer, the distribution shown in Figure 28 meets this criterion.

Similar to the lift-alpha curves results shown in Figure 23, the pressure coefficient plots for the turbulence models at an alpha of 16 degrees were plotted in Figure 29. Results show the Transition SST model to have a fair increase in overall lift production compared to sstk $\omega$ and $k \varepsilon$, which had shown the least. However, based on lift alone, results for the S-A model had produced similar output value.

Therefore, post analysis of the lift, drag curves, along with the velocity contours, and wall shear stress, pressure coefficient plots results for an alpha of 16 degrees suggest that the Transition SST model was the model of choice. To ensure the trends seen for this model were not case specific, alpha values of 17 and 18 degrees were additionally tested (with the exclusion of $k \varepsilon$ as this model produced outliers in comparison to other models tested) and results for both cases were consistent with 16 degrees. 

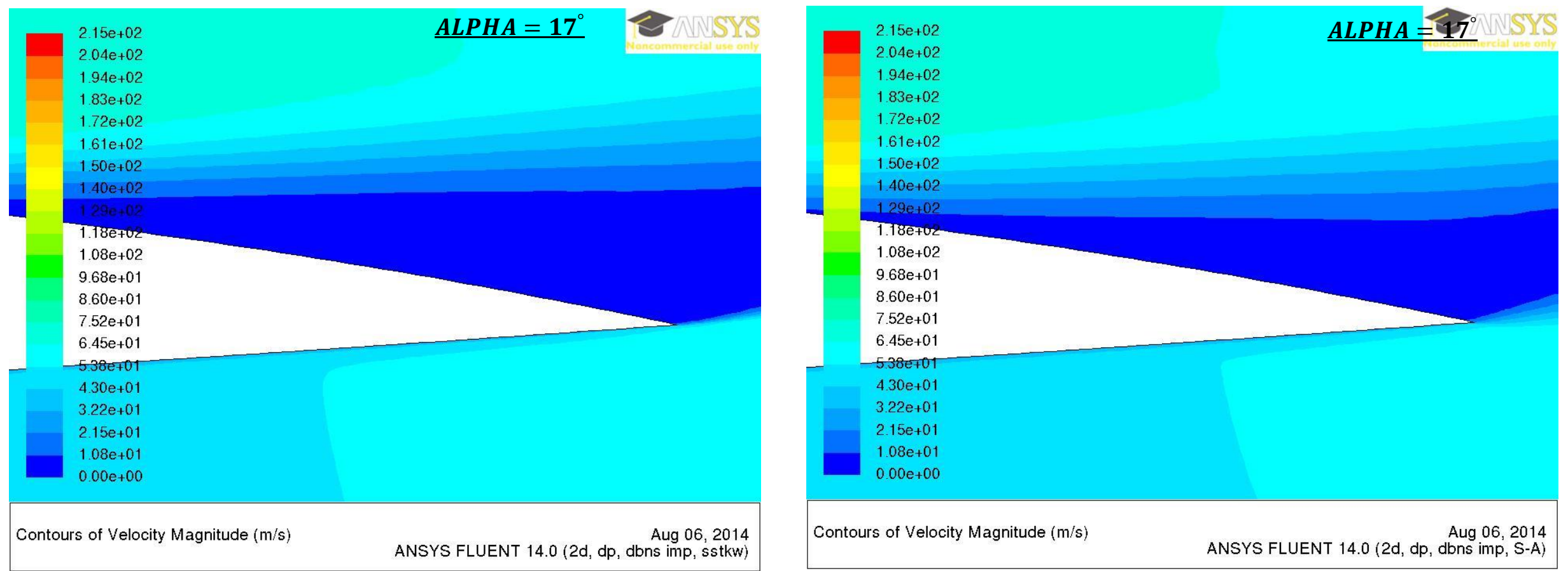

Figure 30: Velocity Contours for Baseline Mesh, Alpha=17, sstkw Model ( $M=0.17, R e=3.79 E 6)$

Figure 31: Velocity Contours for Baseline Mesh, Alpha=17, S-A Model (M=0.17, Re=3.79E6)

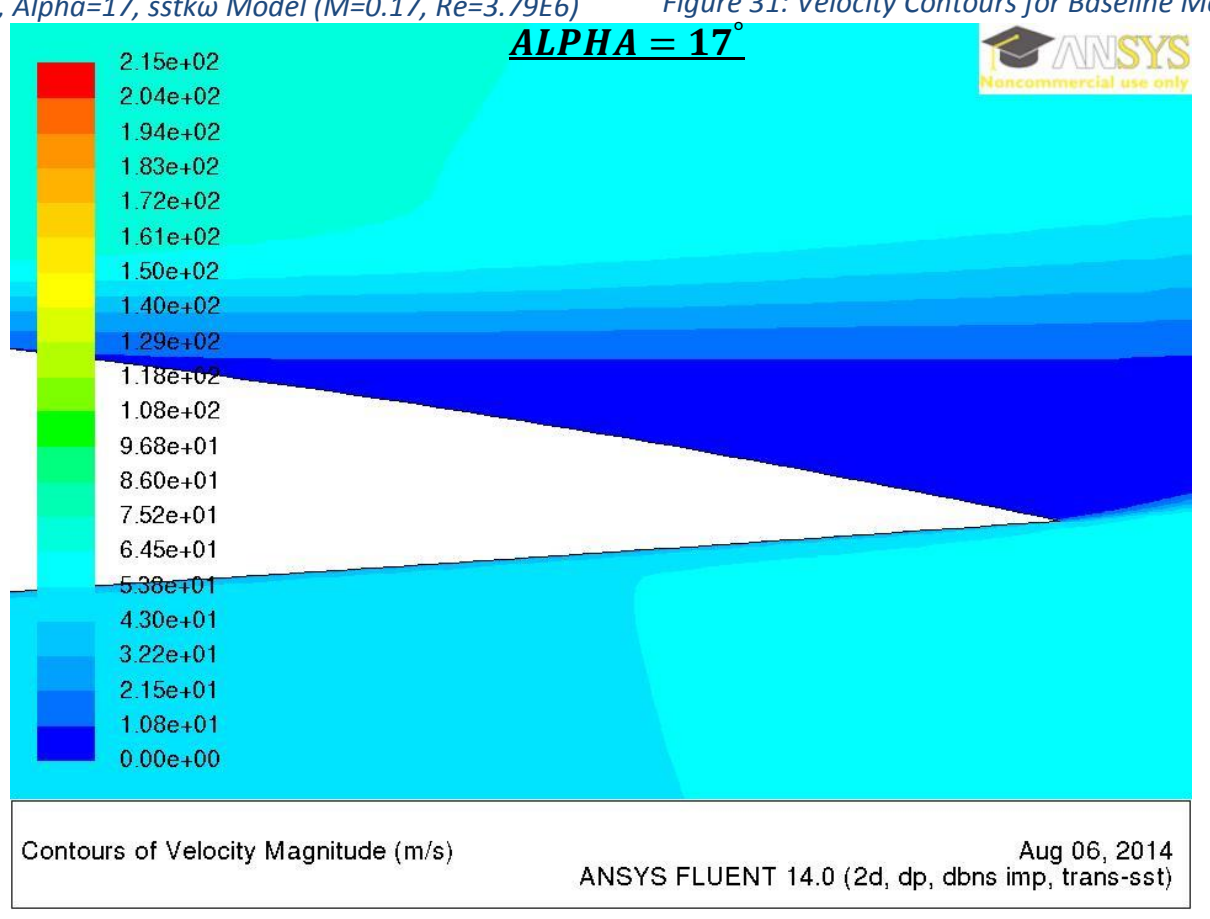

40

Figure 32: Velocity Contours for Baseline Mesh, Alpha=17, Transition SST Model $(M=0.17, \operatorname{Re}=3.79 E 6)$ 


\section{Model Study: Wall Shear Stress Variation}

Baseline (Clean) mesh, $M=0.17, \operatorname{Re}=3.79 E 6, \alpha=17$ degrees

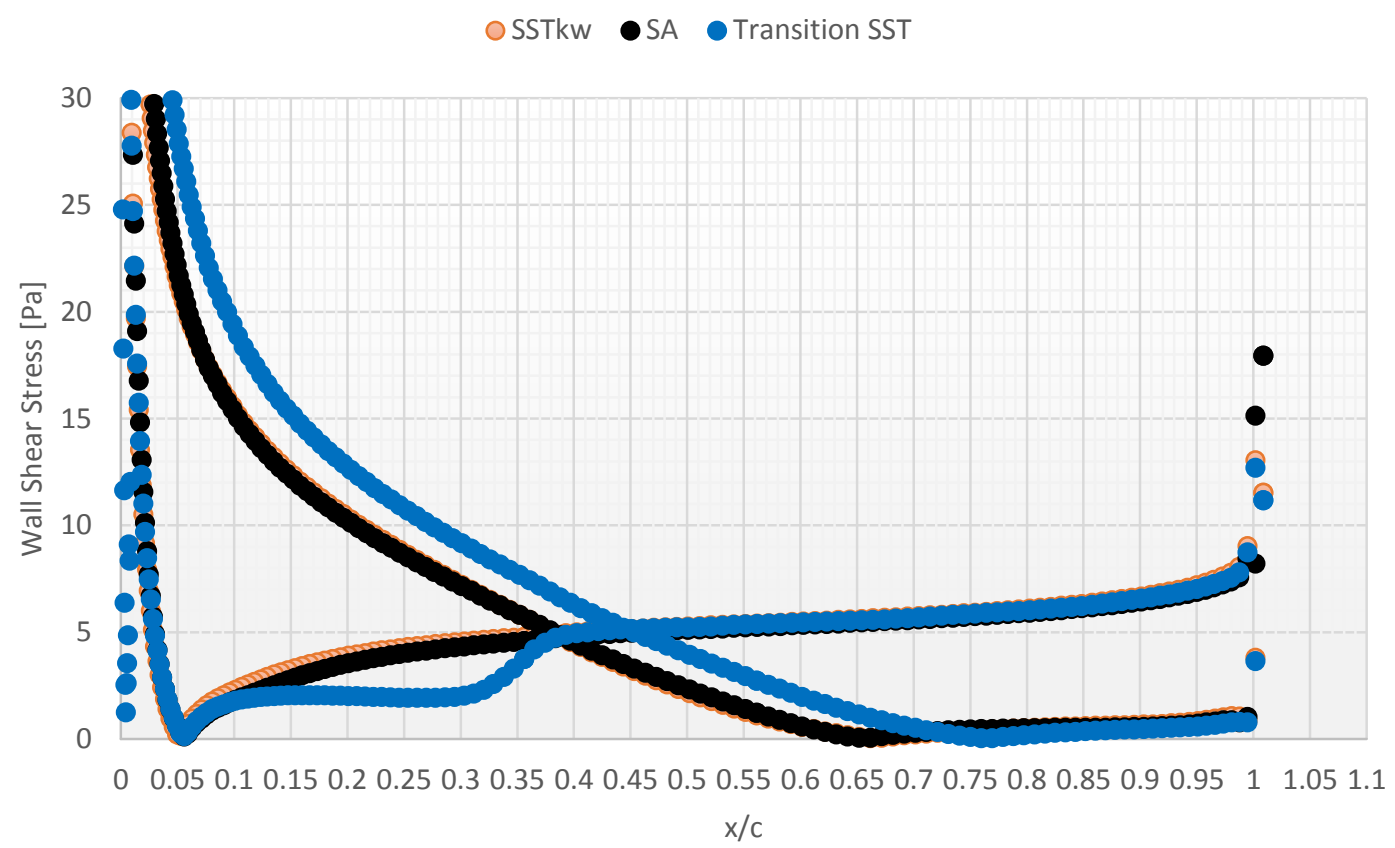

Figure 33: Wall Shear Stress, Baseline Mesh, Alpha=17 (M=0.17, Re=3.79E6)

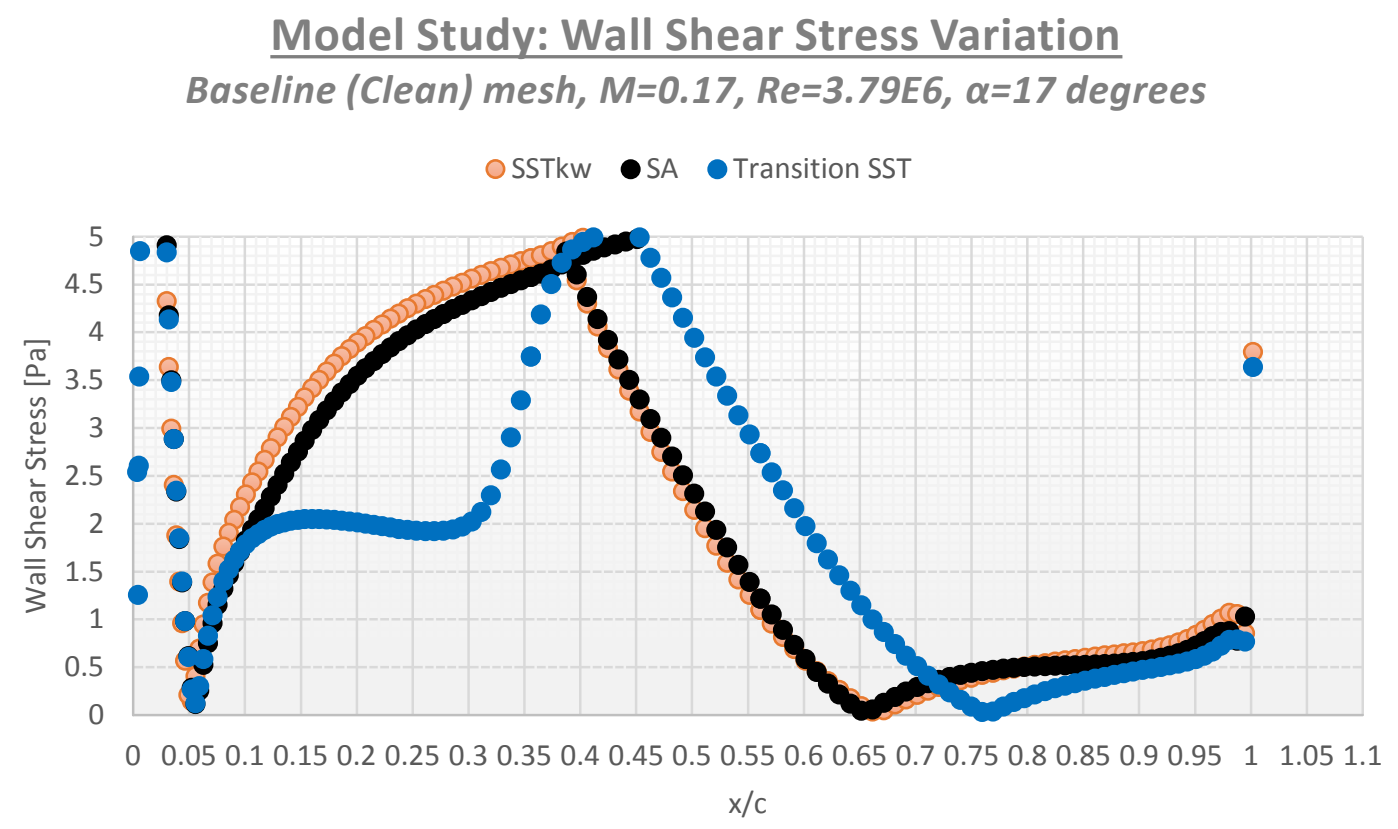

Figure 34: Truncated Wall Shear Stress Plot, Baseline Mesh, Alpha=17 (M=0.17, Re=3.79E6) 


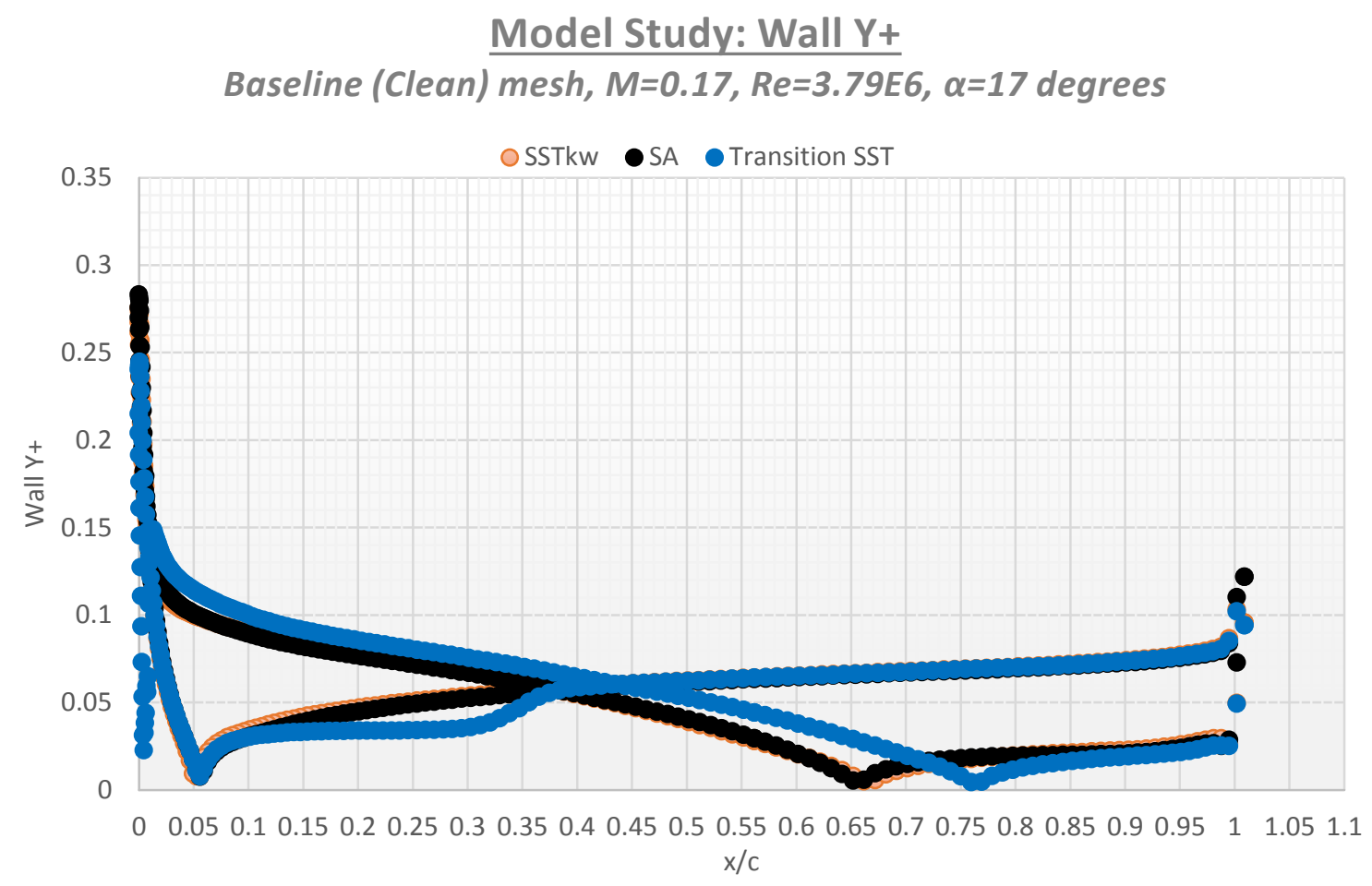

Figure 35: Wall Y+, Baseline Mesh, Alpha=17 $(M=0.17$, Re=3.79E6)

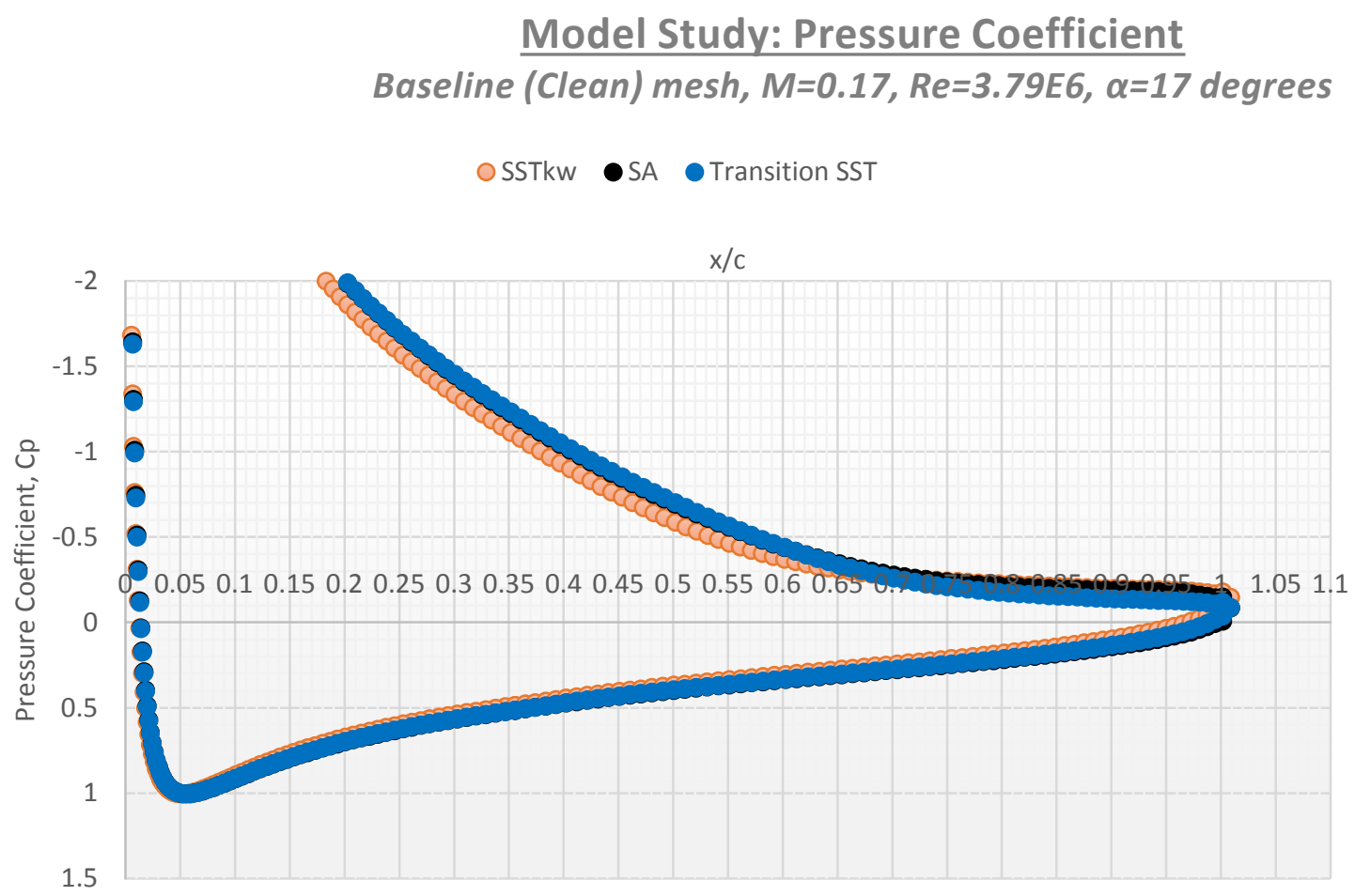

Figure 36: Pressure Coefficient, Baseline Mesh, Alpha=17 (M=0.17, Re=3.79E6) 


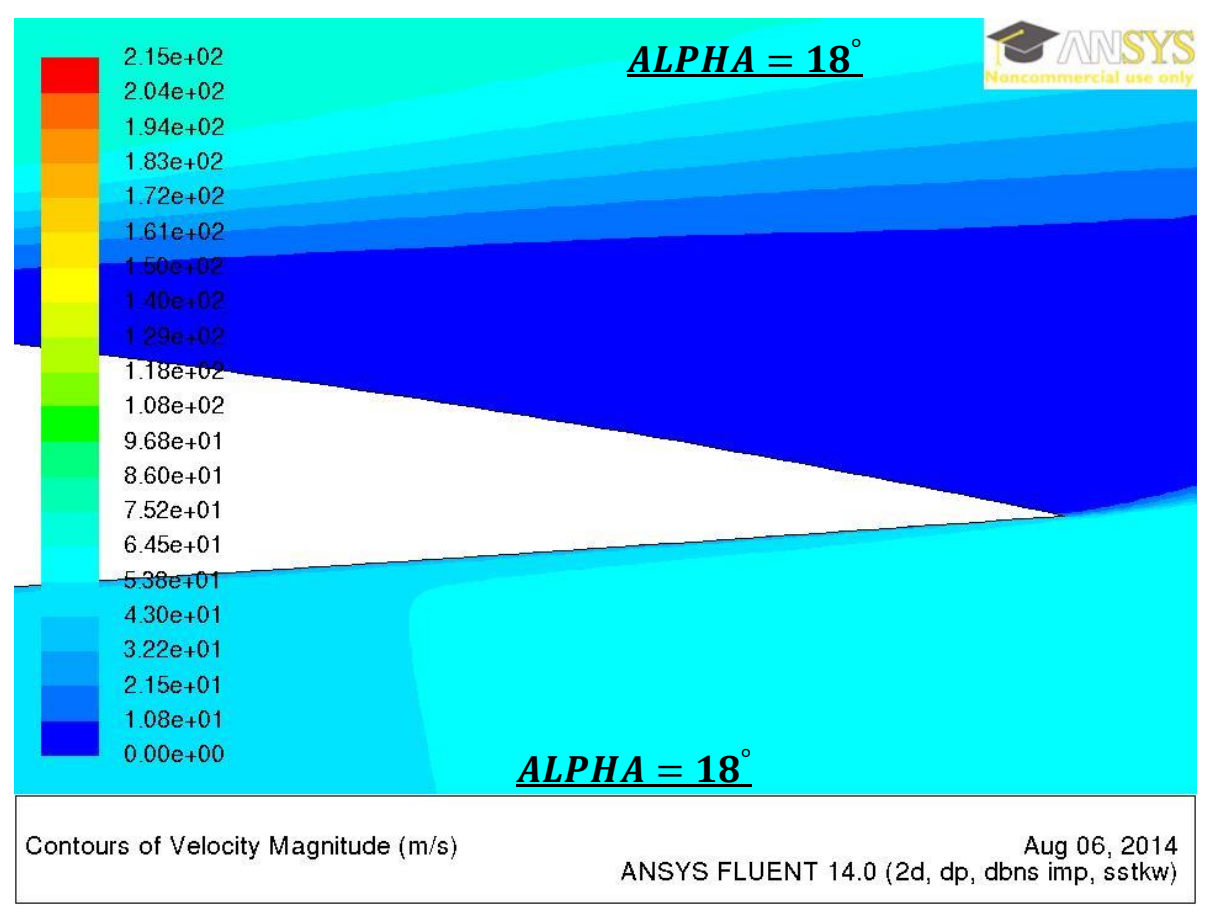

Figure 39: Velocity Contours, Alpha=18, sstkw ( $M=0.17, R e=3.79 e 6)$

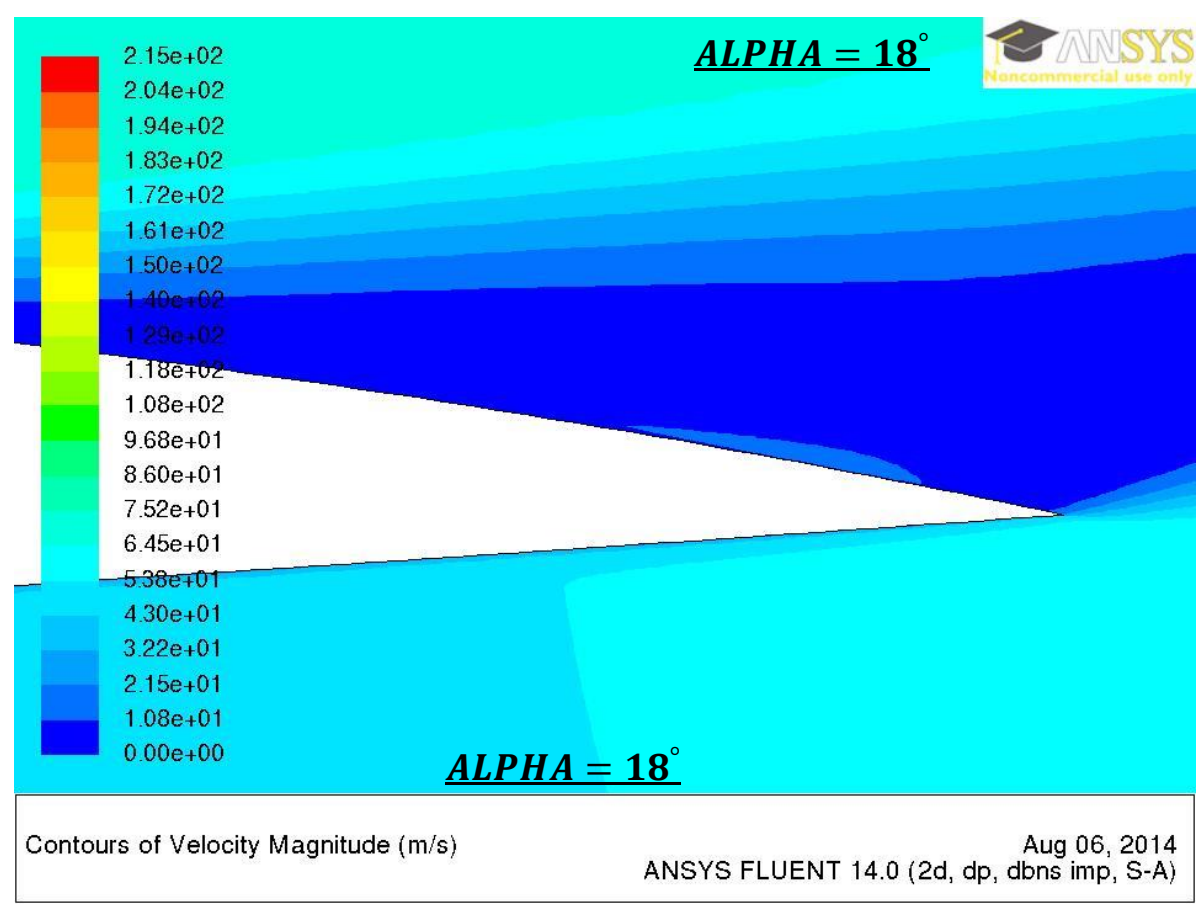

Figure 38: Velocity Contours, Baseline Mesh, Alpha=18, S-A $(M=0.17, R e=3.79 E 6)$

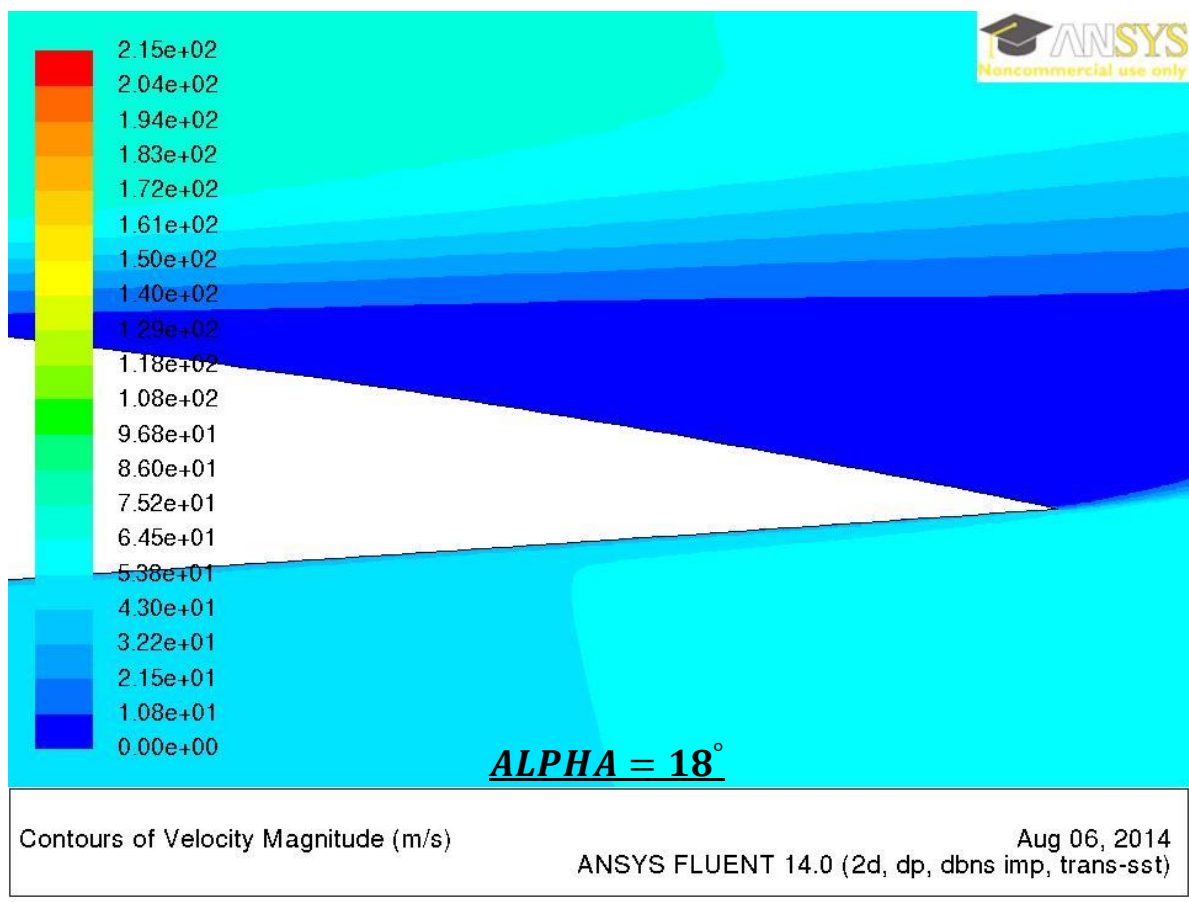

Figure 37: Velocity Contours, Baseline Mesh, Alpha=18, Transition SST Model (M=0.17, Re=3.79E6) 
Model Study: Wall Shear Stress Variation

Baseline (Clean) mesh, $M=0.17, \operatorname{Re}=3.79 \mathrm{E} 6, \alpha=18$ degrees

OSSTkw •SA -Transition SST

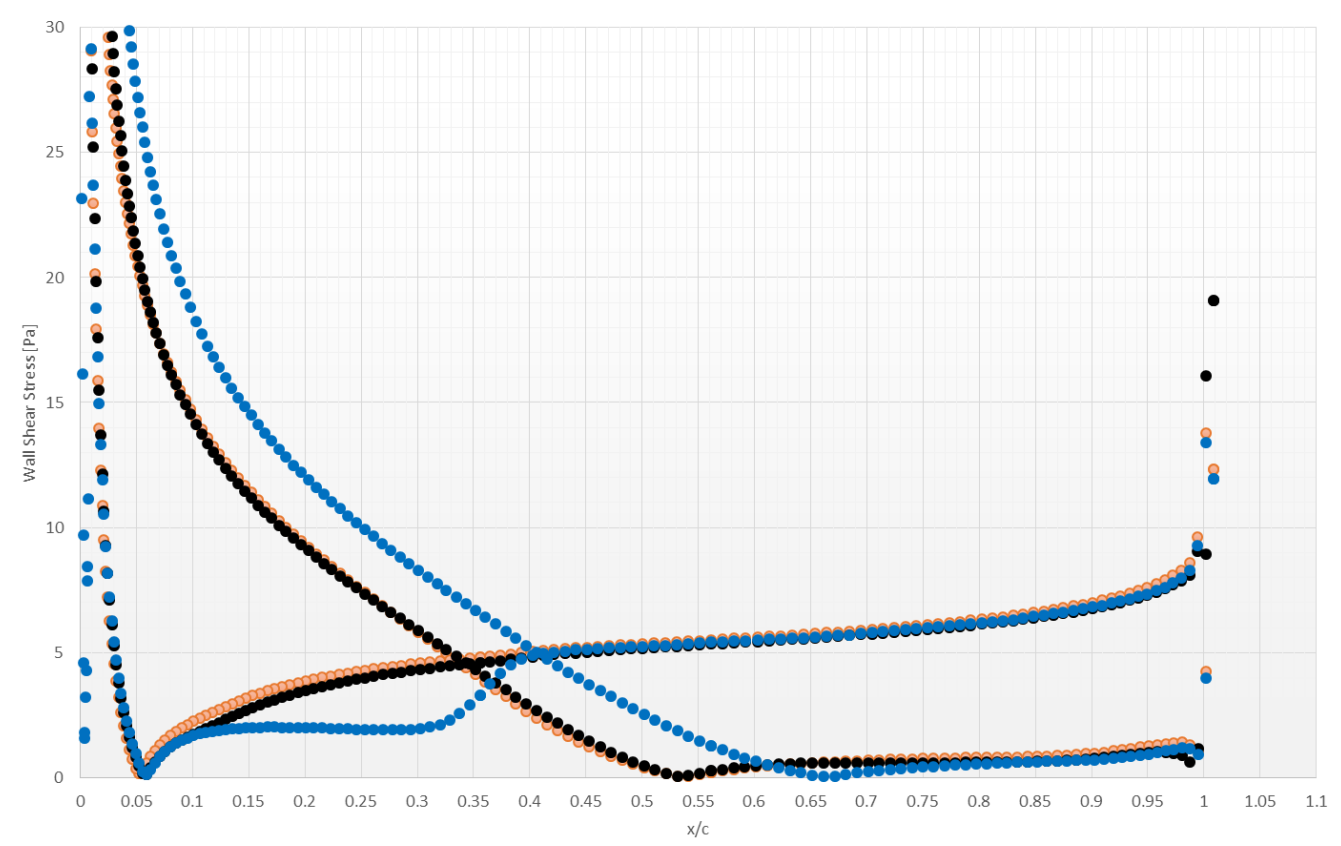

Figure 40: Wall Shear Stress, Baseline Mesh, Alpha=18 $(M=0.17, \operatorname{Re}=3.79 E 6)$

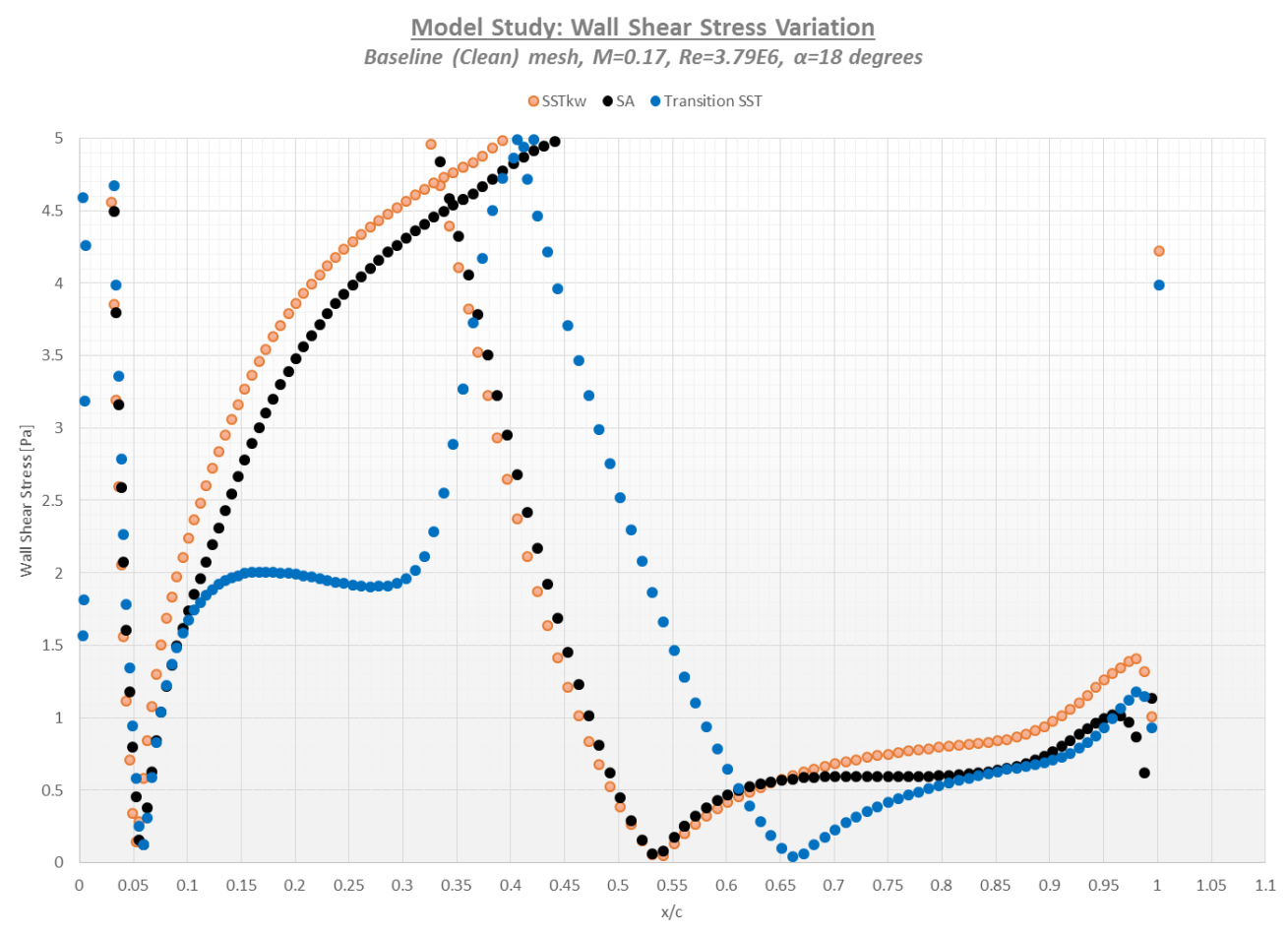

Figure 41: Truncated Wall Shear Stress Plot, Baseline Mesh, Alpha=18 $(M=0.17, R e=3.79 E 6)$ 


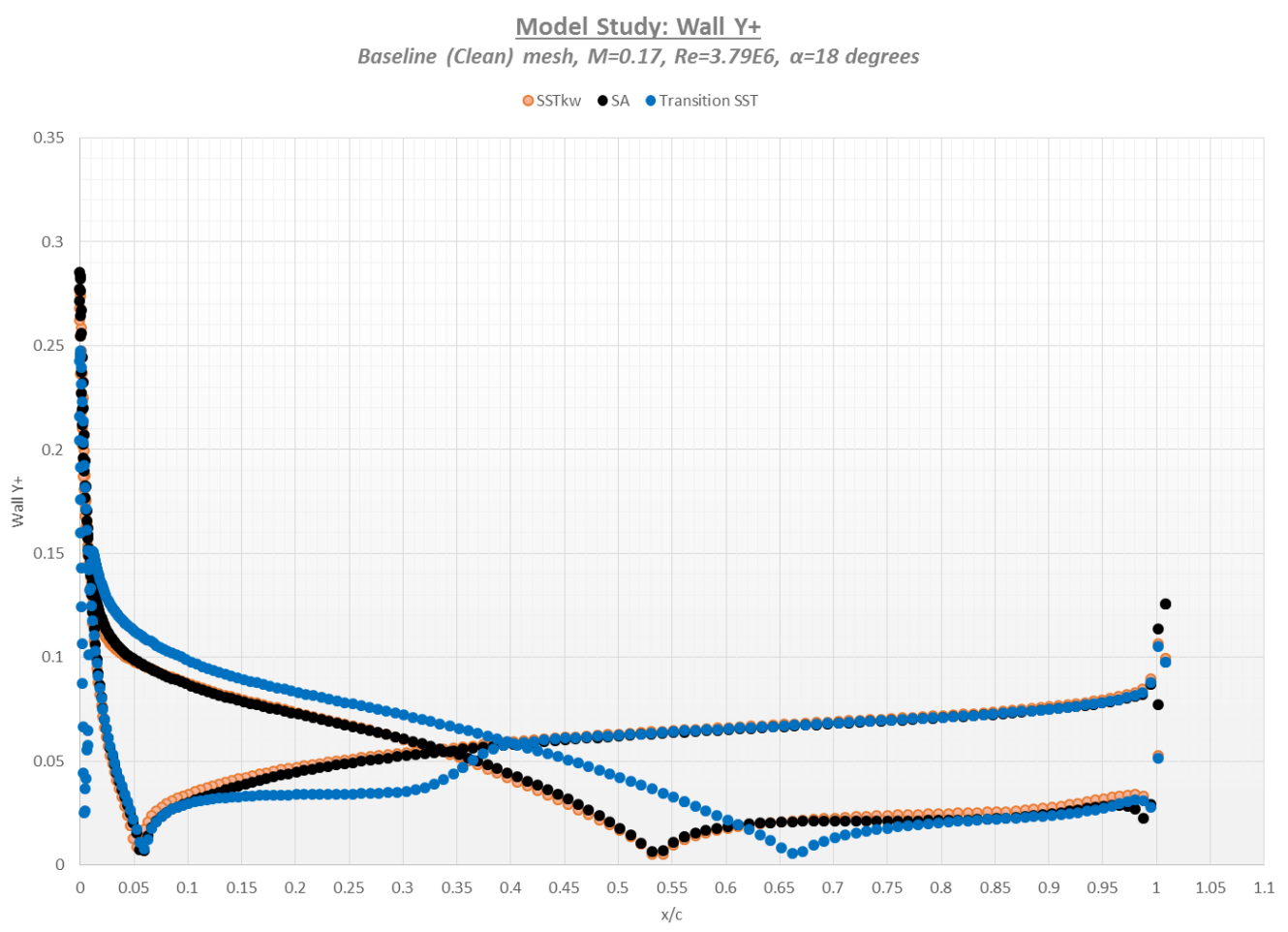

Figure 42: Wall Y + Distribution, Baseline Mesh, Alpha=18 (M=0.17, Re=3.79E6)

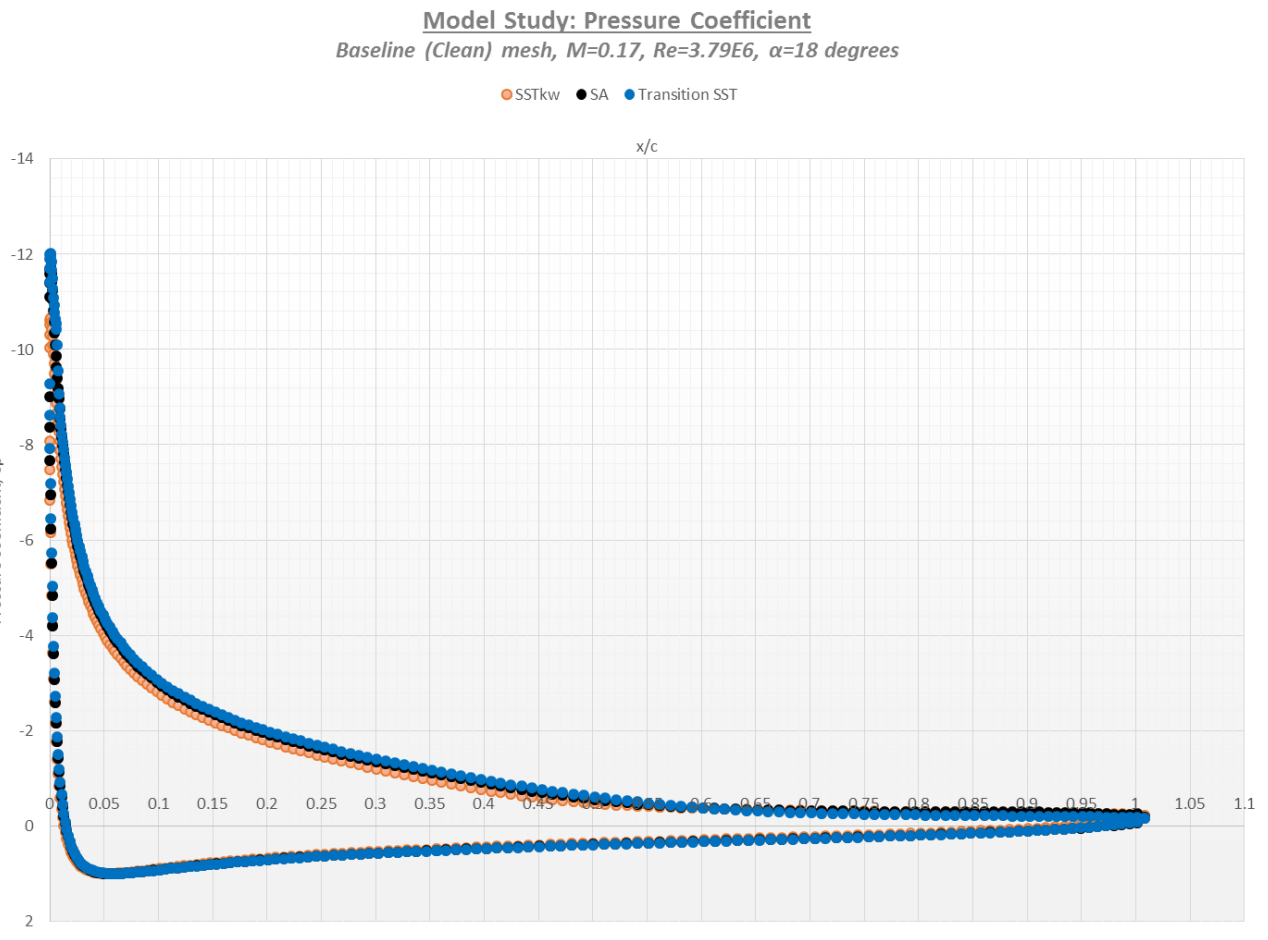

Figure 43: Pressure Coefficient Distribution, Baseline Mesh, Alpha=18 ( $M=0.17, R e=3.79 E 6)$ 
Velocity contour plots for an alpha of 17 degrees can be seen in Figure 30-Figure 32. Similar to the trends seen for an alpha of 16 degrees, Transition SST model (Figure 32) had shown a reduced region of separated flow, in comparison to that seen for sstk $\omega$ and ke models. This finding is seen yet again for the results corresponding to an alpha of 18 degrees seen in Figure 37. In Figure 37 the Transition SST model exhibits the least total area of separated flow near the trailing edge section of the NACA 2412 airfoil. Total surface area affected by separated flow provides an indication of airfoil overall lift generation capability. The least affected should have a larger resultant lift force and as shown in the results the Transition SST model provided the most overall lift as demonstrated to be seen in Figure 36and Figure 43. The majority of lift generated appears to be gained near the leading edge, prior to reaching the $50 \%$ chord location.

Cross comparison of the velocity contours between alpha's 16-18 degrees for the Transition SST model (Figure 25, Figure 32, Figure 37) shows a growing separated flow region with increasing alpha. This expected as pitch is set by the angle of attack, which by definition is a measure of the angle between the free stream velocity and airfoil chord length. Therefore, increasing the airfoil pitch inadvertently transforms the smooth incoming laminar flow along the upper surface of the airfoil to shift to turbulent flow further downstream, in which this region of irregular flow is defined to be the 'wake' region. The point at which the flow transforms from laminar to turbulent is designated as the transition region. There are several factors that influence the shift of the transition regions which include surface roughness, thermal heating, and adverse pressure gradient among many others [18]. Surface roughness, as the name suggests is a qualitative parameter describing the surface wall structure. A high degree of surface irregularity (for example, dirt or debris accumulation on the wall) will create immediate friction between the fluid and surface wall, thereby inducing an early onset of transition to turbulent flow. Thermal heating is directly linked to friction in the form of loss of kinetic energy. Friction between the airfoil wall surface and fluid causes fluid flow velocity to slow down and since velocity is a major function of kinetic energy; therefore kinetic energy decreases. This loss in kinetic energy is expended in the form of thermal heat, which creates a local increase in fluid temperature in comparison to the wall surface [17]. Therefore, due to this temperature gradient, fluid flow will gravitate towards the cooler wall surface which evidently breaks up laminar flow. However, the effects of thermal heating are more significant in supersonic flows in comparison to the subsonic regime, which is the focus of this thesis. A significant factor to flow separation in subsonic flow 
is due to the presence of an adverse pressure gradient. Pressure by definition is a force per unit area and the force present has two forms: pressure drag due to free stream velocity and frictional drag. Frictional drag is the force responsible to work against the movement between the solid wall surface and fluid. It acts to slow down fluid flow opposite to the direction of flow. In saying so, it is due to this that the nominal low pressure zone along upper trailing edge wall surface transitions to a high pressure region, creating what is commonly known as an adverse pressure gradient. Therefore, increasing the pitch angle induces a larger surface area of turbulent flow as viscous forces dominate further upstream. The point at which the adverse pressure gradient dominates the upper surface airfoil section so much so to render the airfoil in effective in lift production is defined to be the point of stall, which is what is seen to occur between 16 and 17 degrees alpha in Figure 25 and Figure 32.

Overall, the wall $y+$ plots for all models tested had shown a maximum wall $y+$ value of less than one, as shown in Figure 35 for an alpha of 17 degrees and Figure 42 for 18 degrees. Therefore, the off wall spacing is such that the wall $y+$ plots are at an acceptable value for the SA and transition SST models.

The wall shear stress plots had shown an over 10\% chord gain in upper surface location of the separation point for the Transition SST model in comparison to sstk $\omega$ and S-A model. At an alpha of 17 degrees, the separation point (Figure 34) for the Transition SST was found to be at approximately $0.75 \%$ chord in comparison to the $0.65 \%$ chord point for sstk $\omega$ and S-A model. Similarly, for an alpha of 18 degrees, Figure 41, the separation point for ssk $\omega$ and S-A was found to be at $0.54 \%$ chord, in comparison to $0.65 \%$ chord seen for Transition SST model. Thereby suggesting the Transition SST model would be the ideal choice for selection.

Therefore, post analysis of the results for alpha values of between 16-18 degrees based on the velocity contours and wall shear stress plots seen collectively, the Transition SST model has shown superior results in lift performance and near wall separation resolution. Hence, the Transition SST model was used as the turbulence model for conduction of the main study. Note no in depth, comprehensive study of turbulent model effects on boundary layer structured behaviour was conducted. Model selection was based on a basic comparison of turbulence model behaviour of stall. 


\subsection{The Grid Independence Study}

The Grid Independence Study was conducted for the purpose of estimating error associated with the nodal distribution in the ideal mesh to be used for the design of the airfoil-spoiler mesh in the main study. The objective was to ensure that the ideal baseline mesh was at its most optimal form i.e. within a reasonable error band to yield significant or meaningful results. The study was carried out by applying Richardson's Extrapolation which dictates the use of multiple mesh refinements: course, medium and fine mesh [19]. The theory dictates that grid solutions denoted by the function $\mathrm{f}$, have a Taylor series representation using the grid spacing, h shown in the following form:

$$
f=f_{\text {exact }}+g_{1} h+g_{2} h^{2}+g_{3} h^{3}+\cdots
$$

Where Richardson's Extrapolation for $\mathrm{f}_{\text {exact }}$ is approximated by

$$
\begin{aligned}
& f_{\text {exact }} \cong f_{1}+\frac{f_{1}-f_{2}}{r^{p}-1} \\
& r=\text { grid spacings }=\frac{h_{2}}{h_{1}} \text { or } \frac{h_{3}}{h_{2}}
\end{aligned}
$$

According to the theory, Richardson's Extrapolation estimation for $\mathrm{f}_{\text {exact }}$ is valid provided the solutions, $\mathrm{f}_{1}, \mathrm{f}_{2}$ etc. are within an asymptotic range. The most common techniques to apply this theory involve grid doubling or halving procedures. For this study, Richardson's Extrapolation was applied by doubling the nodes and halving the spacing's moving from a course to medium (ideal) to fine mesh. Therefore, the term representing grid spacing, $r$, is equal to 2 where $h_{1}$ represents the fine grid and $h_{3}$ the course grid. The order of convergence, represented by $p$, is theoretically taken to be 2 since the nodal distribution was doubled each time in the creation of the course to medium mesh, and medium to fine mesh. Past experience dictates that the lift coefficient is resolved with a high degree of fidelity within Computational Fluid Dynamics as it is a resultant of free stream pressure, with little or no influence of the boundary layer. However, that being said, the lift coefficient is strong correlated to the total number of nodes present in the mesh. Increasing the number of nodes moving from a course to medium mesh for example provides various lift coefficient solutions in which it is more likely that the grid with the most 
nodes will have the higher numerical value, and be assumed to represent the solution for the case studied with a high degree of confidence. Therefore, the objective in using Richardson's Extrapolation was to determine analytically if the solutions for lift coefficient followed an order of convergence of approximately two, and an estimation of the extrapolation error between the three meshes tested. An alpha value of 16 degrees was used for this study as it is a high pitch angle and point on the lift coefficient-alpha curve prior to stall. Since the study is centered on alpha cases past stall, this case would provide an adequate representation of the error involved in resolving high alphas against the ideal (medium mesh) studied.

An analytical solution for $\mathrm{p}$, the order of convergence between three meshes tested can be determined by the following relations:

$$
p=\frac{\ln \left(\frac{f_{3}-f_{2}}{f_{2}-f_{1}}\right)}{\ln (r)}
$$

The Grid Convergence Index (GCI) is an estimation of the error between the meshes tested. The GCI between the two finest meshes (fine and medium where the subscript 1 represents the fine mesh) is represented by the following equation:

$$
G C I_{12}=\text { FACTOR OF SAFETY } * \frac{\left|\frac{f_{2}-f_{1}}{f_{1}}\right|}{r^{p}-1}
$$

The GCI, between the medium and course mesh in comparison is found to be the following:

$$
G C I_{23}=\text { FACTOR OF SAFETY } * \frac{\left|\frac{f_{3}-f_{2}}{f_{2}}\right|}{r^{p}-1}
$$

According to Patrick J. Roache [19], grid convergence studies with a minimum of three grid should be applied for Reynolds Number of greater than one.. Three grid convergence studies use a factor of saftey of approximately 1.25, whereas grid studies using two meshes (two finest in comparison to the three meshes: course, medium and fine) should use a factor of safety of 3 [19]. Therefore, the goal of the study was to determine whether the solutions for the Taylor series representation are within an asymptotic range of one.

$$
\text { asymptotic value } \approx \frac{G C I_{23}}{G C I_{12} * r^{p}} \approx 1
$$


The following are the mesh distribution near the NACA 2412 wall surface for visualization of the nodal doubling and spacing reduction by a factor of 0.5 moving from a course, medium and fine mesh.

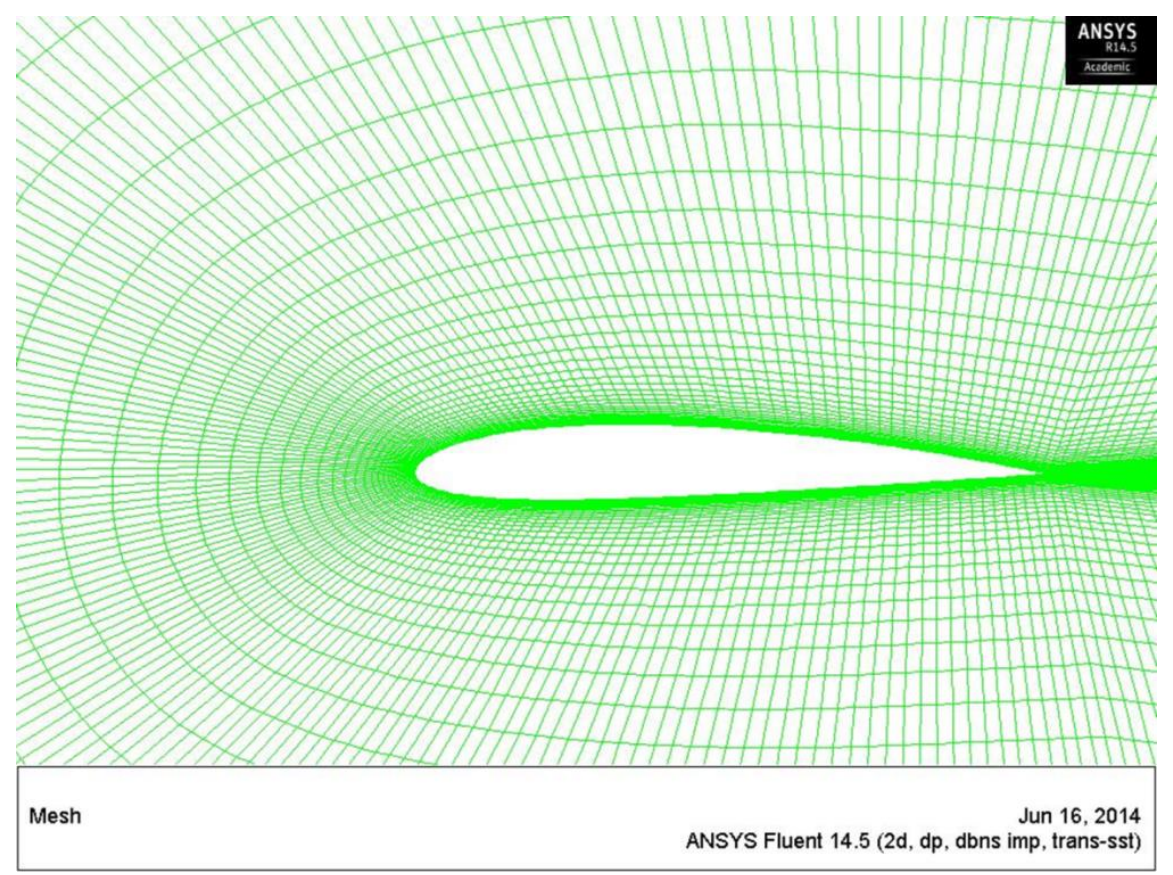

Figure 44: NACA2412 Baseline, Course Mesh, 25,000 Nodes, Transition SST

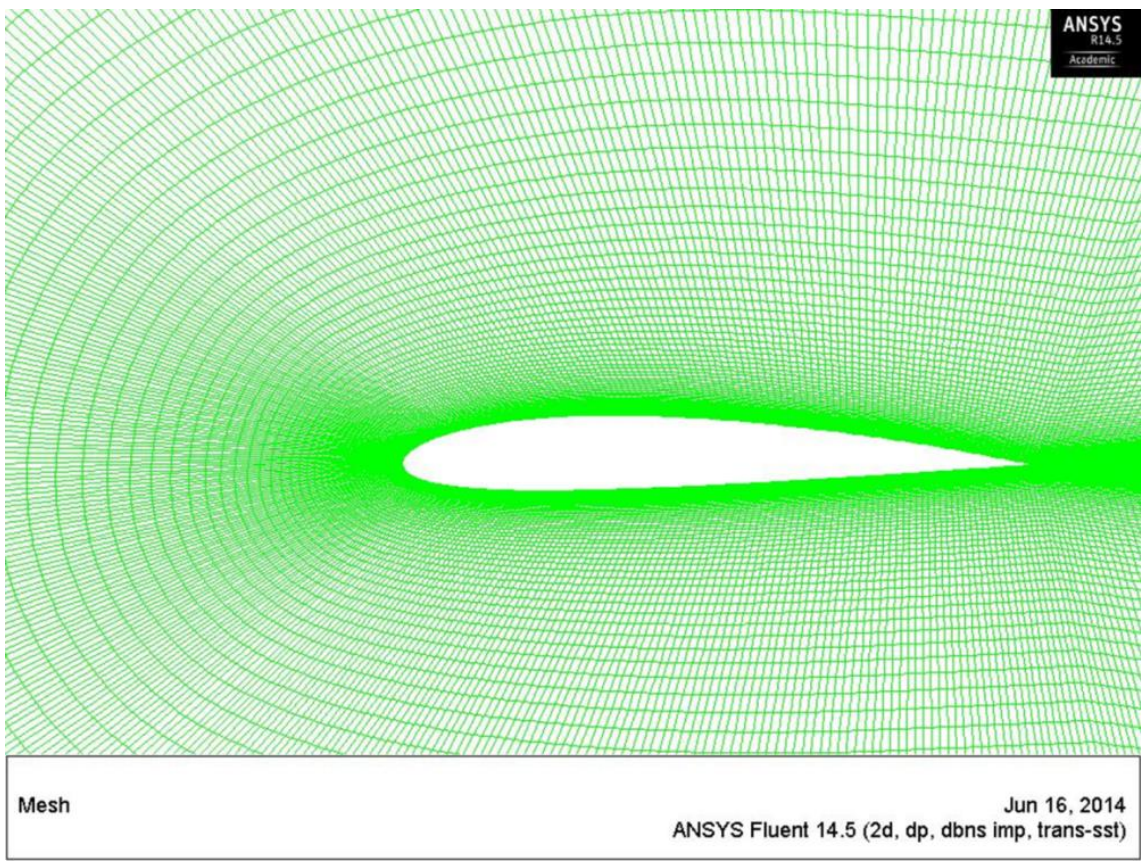

Figure 45: NACA2412 Medium (Ideal) Baseline Mesh, 100,000 Nodes, Transition SST 


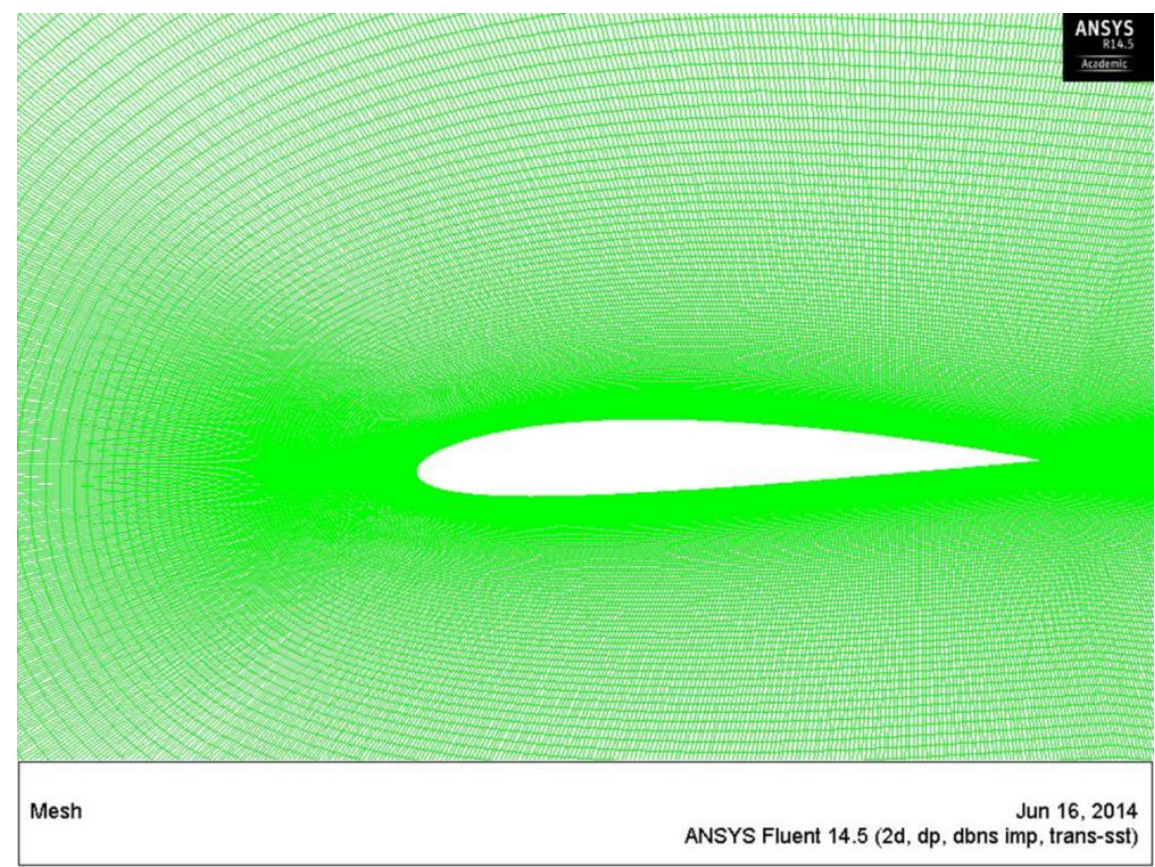

Figure 46: NACA 2412 Fine Baseline Mesh, 400,000 Nodes, Transition SST

Results for an alpha value of 16 degrees for the course, medium and fine mesh were found to be the following:

Table 6: Grid Independent Study Results for Baseline Mesh, Alpha=16

\begin{tabular}{|ccccc|}
\hline Mesh & $\begin{array}{c}\text { Solution } \\
\text { Parameter }\end{array}$ & $\begin{array}{c}\text { Lift } \\
\text { Coefficient }\end{array}$ & Drag Coefficient & $\begin{array}{c}\text { Moment } \\
\text { Coefficient }\end{array}$ \\
\hline Course ( 25K Nodes) & $\mathrm{f}_{3}$ & 1.689 & 0.0332 & -0.0144 \\
\hline $\begin{array}{c}\text { Medium ( 100K } \\
\text { Nodes) }\end{array}$ & $\mathrm{f}_{2}$ & 1.7025 & 0.0331 & -0.0149 \\
\hline Fine ( 400K Nodes) & & & & -0.0152 \\
\hline
\end{tabular}


Convergence was assessed by ensuring the residuals reduced by an order of $\sim 1 \mathrm{e}-03$ to $1 \mathrm{e}-04$ in magnitude from the initial start of the case. Point solutions for the lift and drag coefficients were monitored until steady state solutions were attained in which both the lift and drag were seen to remain constant with little or no influence in value with increasing run iterations. For validation purposes, results for the lift and drag coefficient across the course, medium and fine mesh were collected from point monitoring and can be seen in the following figures:

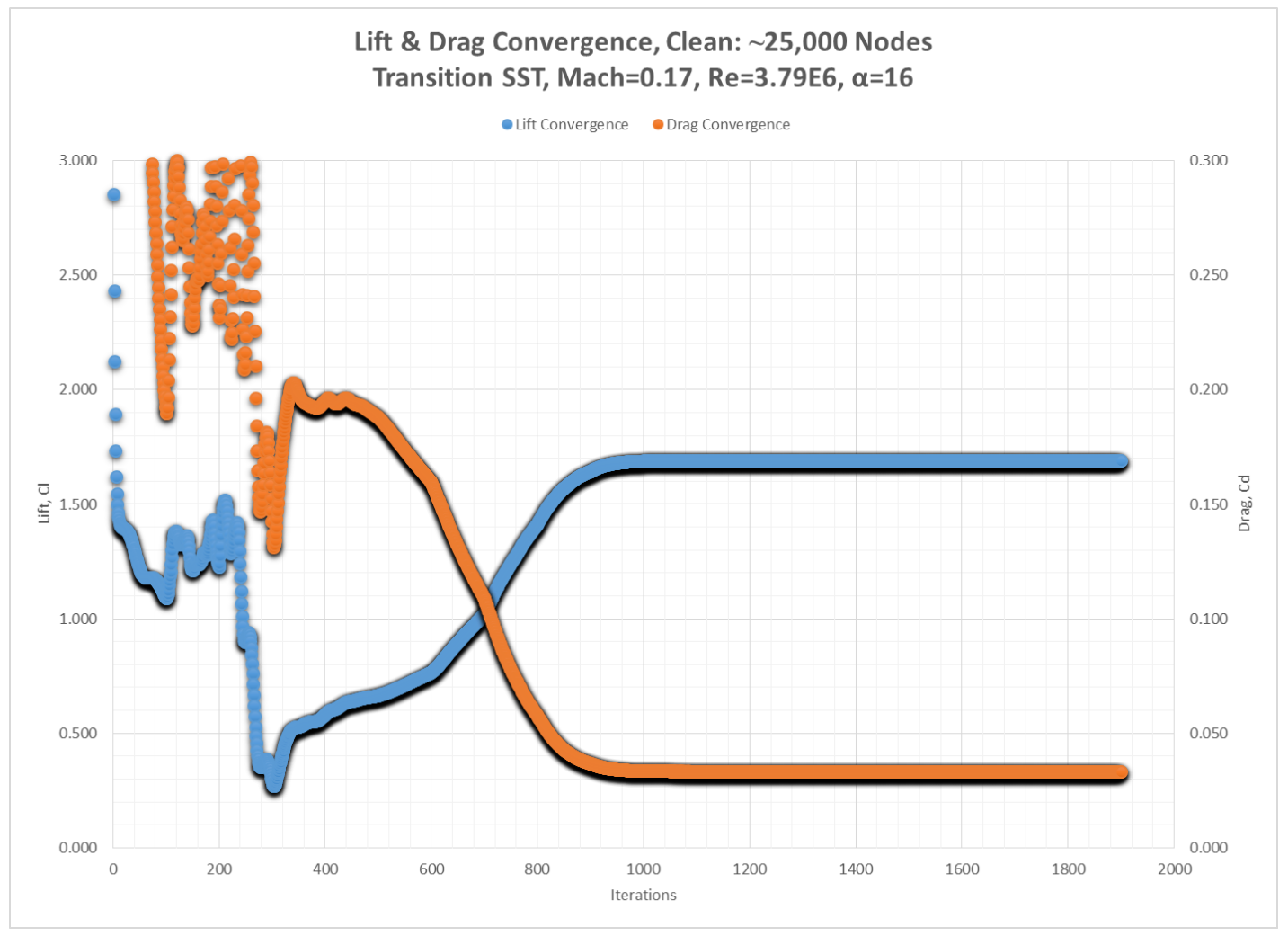

Figure 47: Course Mesh, 25K Nodes, Transition SST, Alpha=16 


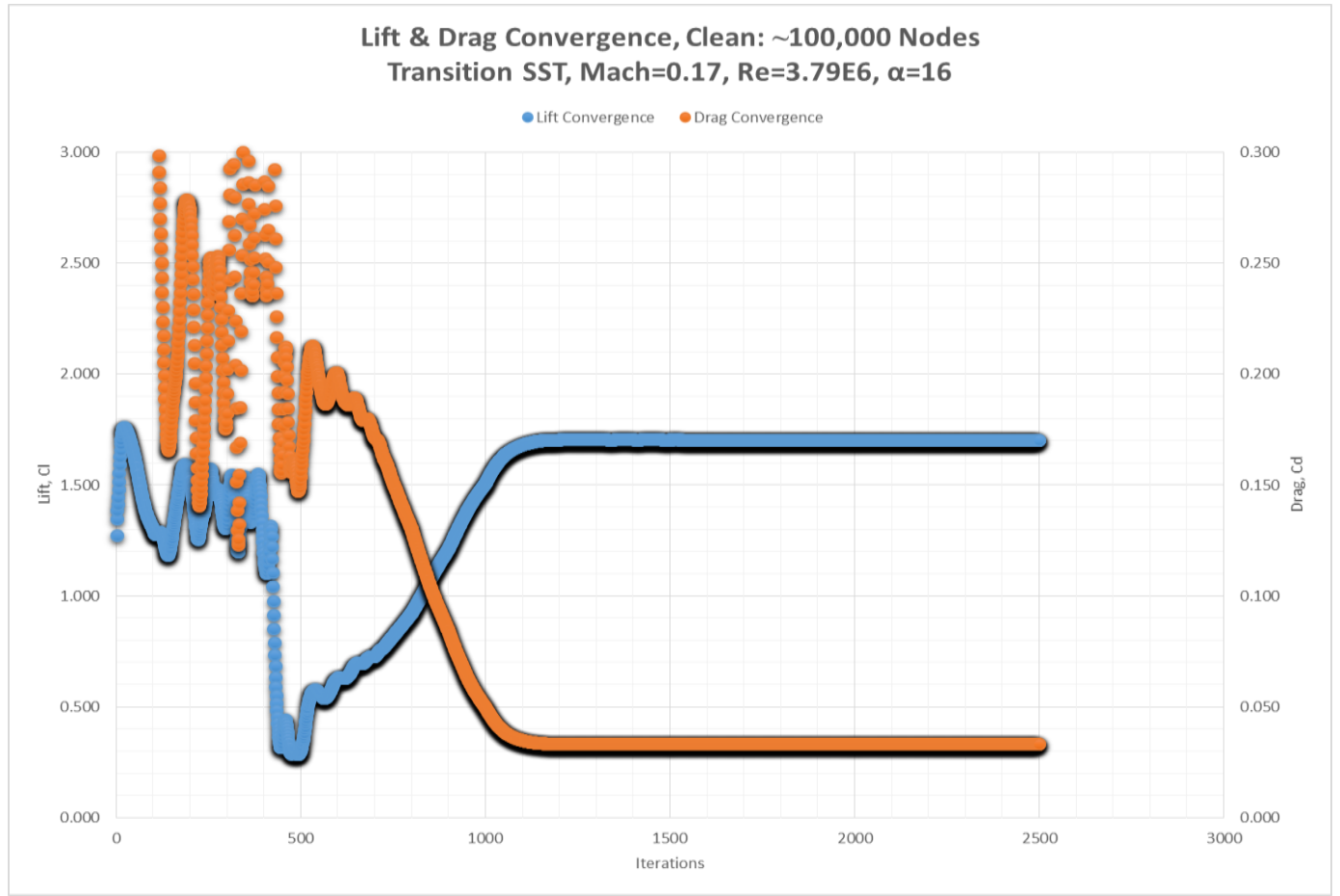

Figure 48: Medium Mesh (Ideal), 100K Nodes, Transition SST, Alpha=16

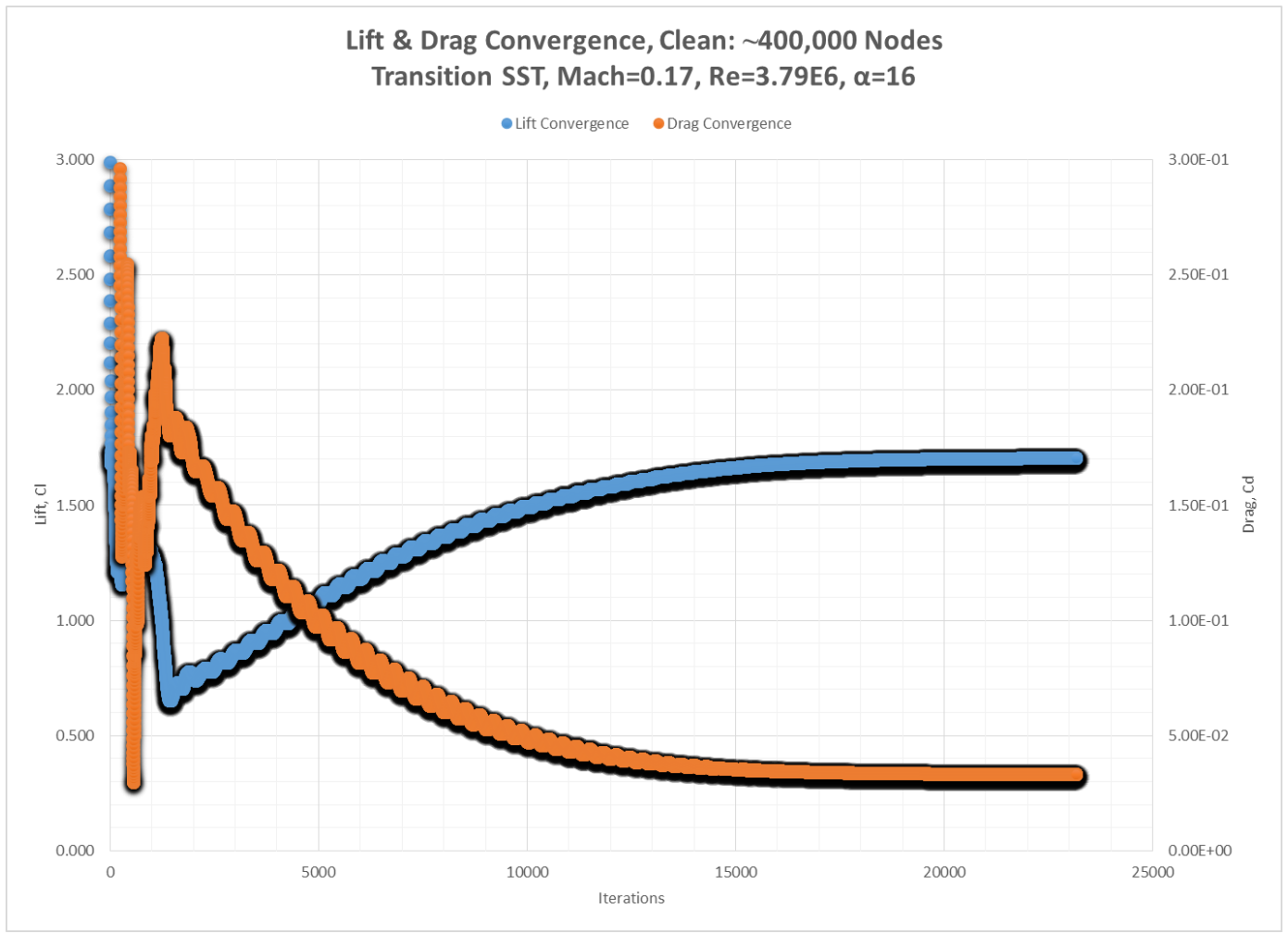

Figure 49: Fine Mesh, 400K Nodes, Transition SST, Alpha=16 


\subsubsection{Grid Independent Study Calculations}

The following was the analytical solution to Richardson's Extrapolation Theory for the Grid Convergence Index Error estimation and asymptotic convergence value for an alpha case of 16 degrees.

\subsubsection{Grid Convergence Study using three Grids for Analysis}

$$
\begin{gathered}
\text { Grid Spacing: } r=\frac{h_{2}}{h_{1}}=\frac{2 h_{1}}{h_{1}}=2 \\
\text { Order of Convergence, } P=\frac{\ln \left(\frac{f_{3}-f_{2}}{f_{2}-f_{1}}\right)}{\ln (r)}=\approx 2.169
\end{gathered}
$$

Richardson'Estimation for $f_{\text {exact }} \cong f_{1}+\frac{f_{1}-f_{2}}{r^{p}-1}=\approx 1.706$

$$
\begin{gathered}
G C I_{12}=\text { Factor of Safety } \frac{\left|\frac{f_{2}-f_{1}}{f_{1}}\right|}{r^{p}-1}=6.28 e-04 \\
G C I_{23}=\text { Factor of Safety } \frac{\left|\frac{f_{3}-f_{2}}{f_{2}}\right|}{r^{p}-1}=2.85 e-03 \\
\text { asymptotic value } \approx \frac{G C I_{23}}{G C I_{12} r^{p}} \approx 1.009
\end{gathered}
$$

Therefore, the asymptotic estimate was found to be approximately one, in which case Richardson's estimate for the lift coefficient for an alpha case of 16 degrees of 1.706 was deemed analytically valid. 
The Grid Convergence Index between the two finest meshes was determined to have an estimated error of less than one percent (also seen between the two course meshes). Thus, the medium mesh was deemed suitable as a platform for collection of the baseline study, as well as serve as the template for mesh generation of the airfoil-spoiler geometry to maintain both consistency and suitable, based on a computational grid error of less than one. In addition, the medium mesh has a total of around 100 thousand nodes, that is "fine" enough to be able to investigate a large number of spoiler cases with a quick and reasonable numerical run time. 


\section{Chapter 4}

\section{Spoiler Performance Study}

\subsection{Spoiler Performance Results}

Information gathered from the grid independence provides key details on best practices for spoiler mesh generation which indicated that the medium mesh size is the ideal layout to use as the spoiler template. In addition, the model selection study provided key solver information for determining the most ideal turbulence model, which was determined to be the Transition SST model. Therefore, both studies served as the foundation on which the spoiler study was carried out. Based on literature review, and guidance from Mr. Alex Tsoulis and Mr. Neal A. Harrison [15], it was found that ideally the spoiler structure is located between $65-70 \%$ leading edge chord location, therefore, $70 \%$ leading edge location served as the maximum aft location for the study. For comparison purposes, $60 \%$ leading edge location was also tested to further understand the change in physics from moving to a location slightly forward, from the conventional $65 \%$ leading edge position. As mentioned earlier, the spoiler length was taken as $10 \%$ chord (based on literature review and suggestions from Mr. Alex Tsoulis [15]) and spoiler deflections of 4 and 10 degrees were tested across various alpha values. The following section presents the results collected for the aerodynamic performance with and without spoiler deflection: 


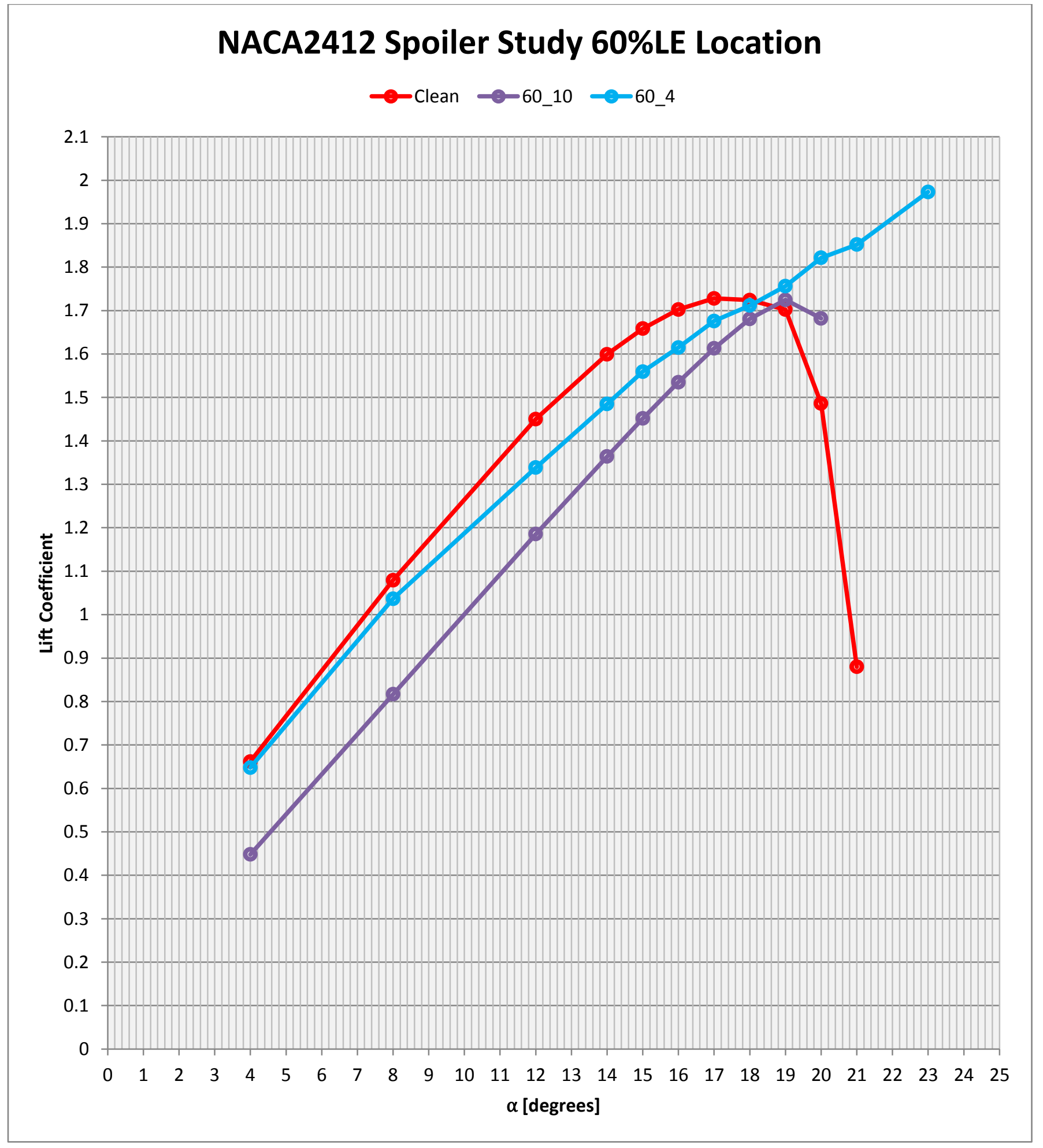

Figure 50: NACA 2412 Spoiler Study Lift Coefficient, Alpha Results, 60\% LE Location 


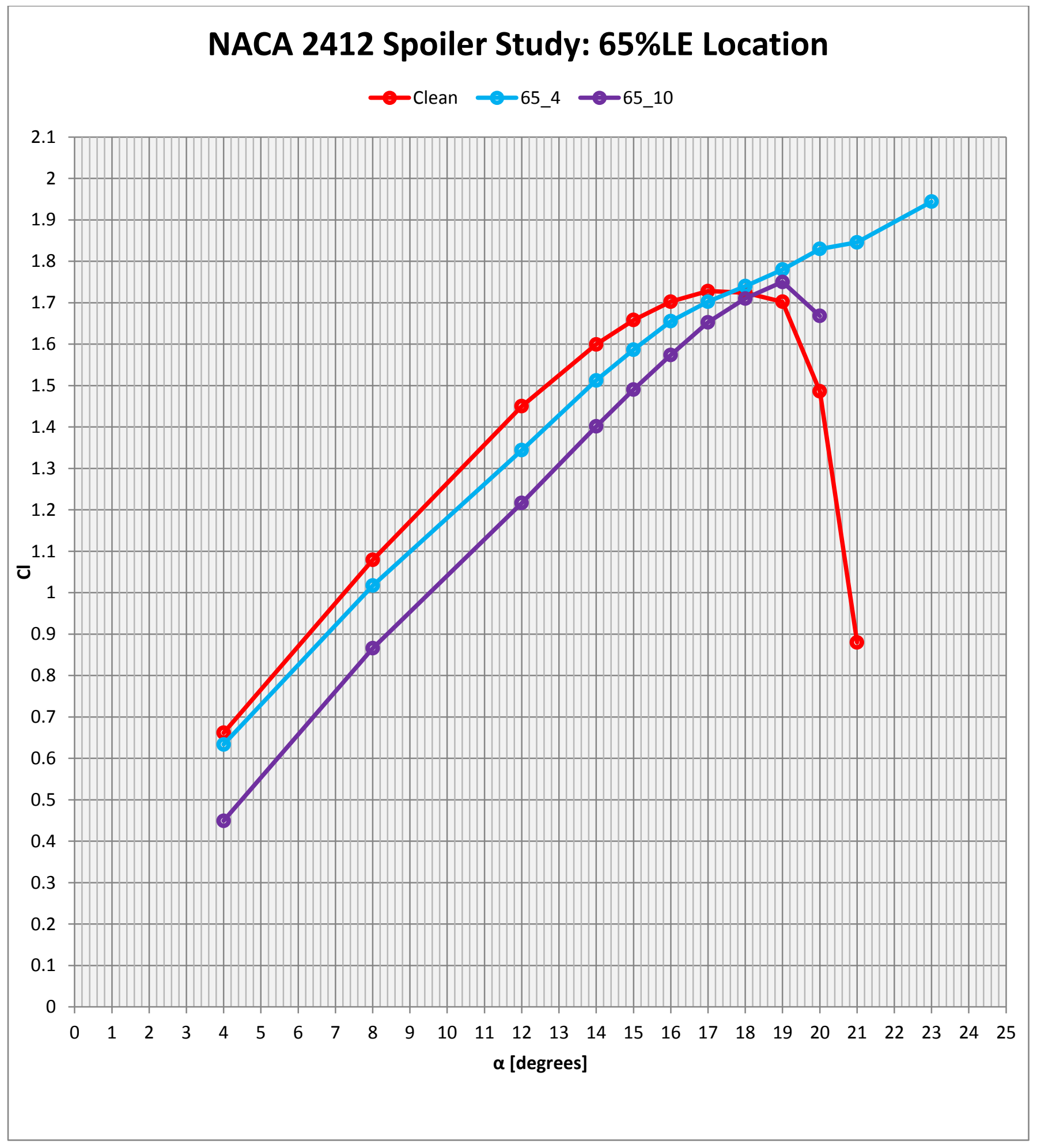

Figure 51: NACA 2412 Spoiler Study Lift Coefficient, Alpha results, 65\% LE location 


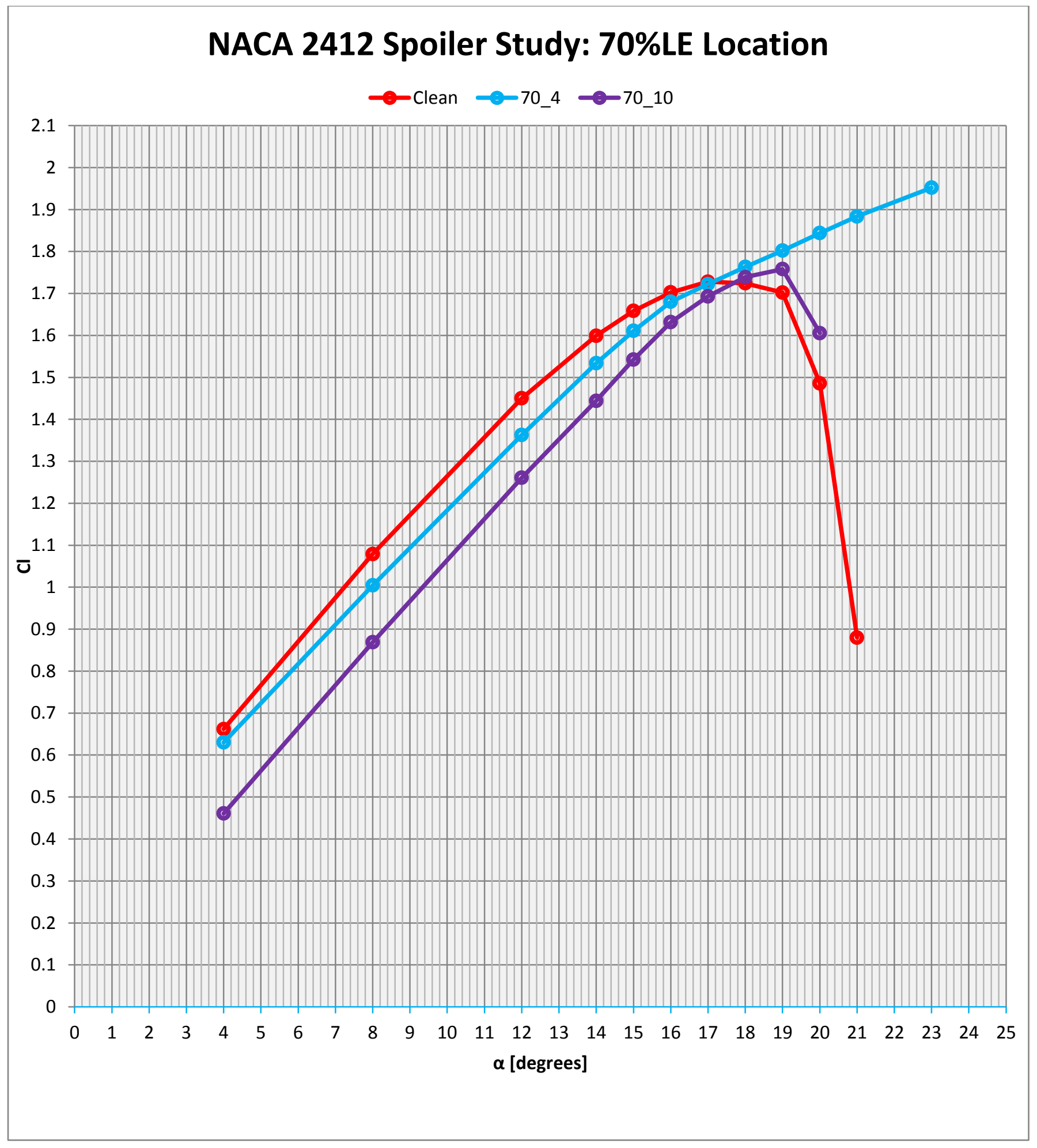

Figure 52: NACA 2412 Spoiler Study Lift Coefficient, Alpha results, 70\% LE location 
As seen in Figure 50,Figure 51, and, Figure 52, the lift coefficient for all upper surface spoiler locations showed a positive lift gain past the baseline stall point of 17 degrees (represented by the red curve) as a result of the incorporation of the 4 and 10 degree spoiler deflections. This finding is pivotal as it clearly demonstrates the spoiler performance in mitigating stall. Specifically, the lift-alpha curve at the $60 \%$ location in Figure 50, the curve corresponding to a spoiler deflection angle of 4 degrees had shown to push the baseline stall point of 17 degrees, to past 21 degrees; that is an additional 4 degrees of contingency. Similarly, the positive gain in lift was seen as an increase in aerodynamic efficiency. Aerodynamic efficiency was taken to be the ratio between the lift coefficient and the drag coefficient.

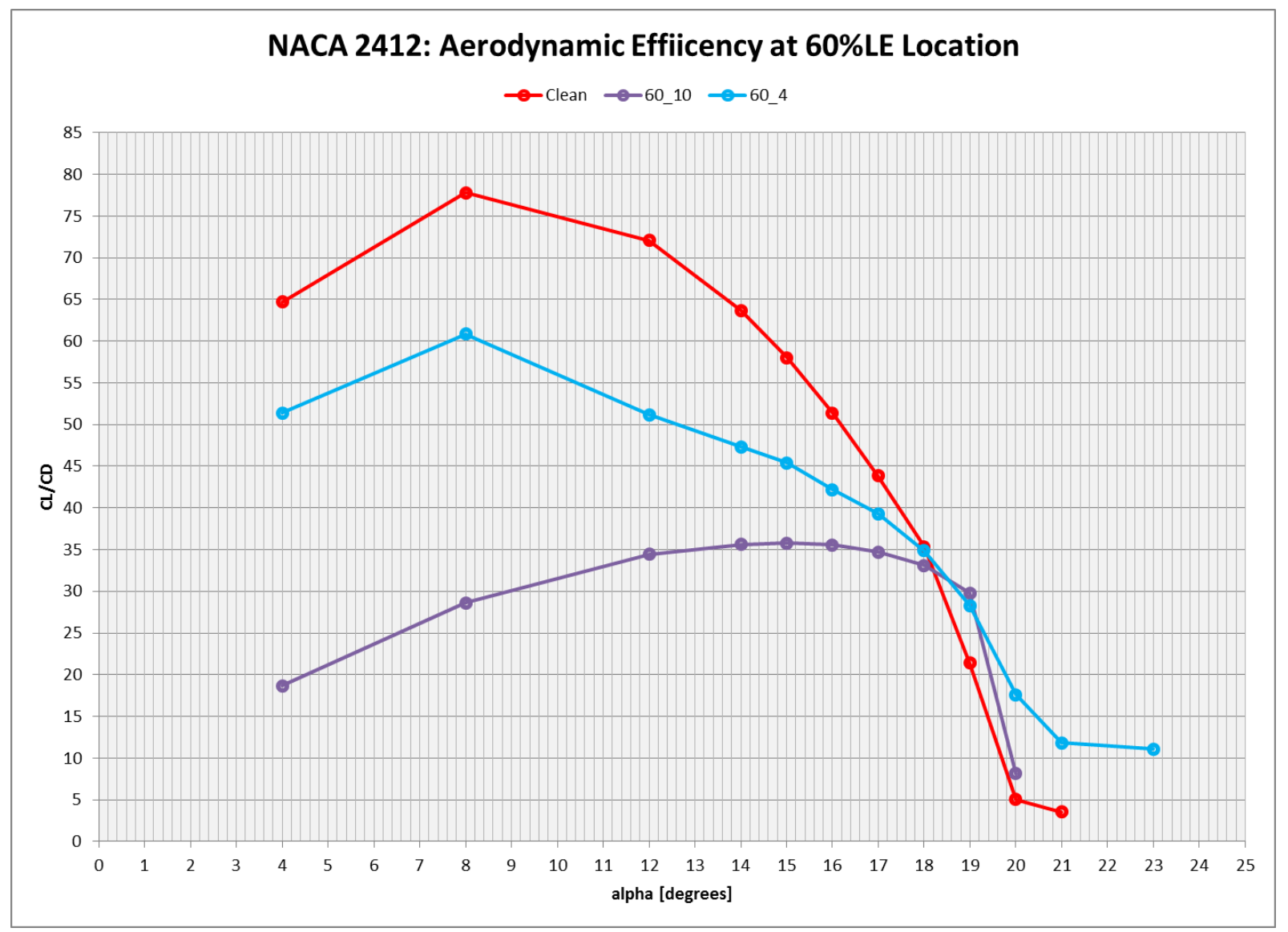

Figure 53: NACA 2421 Spoiler Study, CL/CD at 60\% LE location 


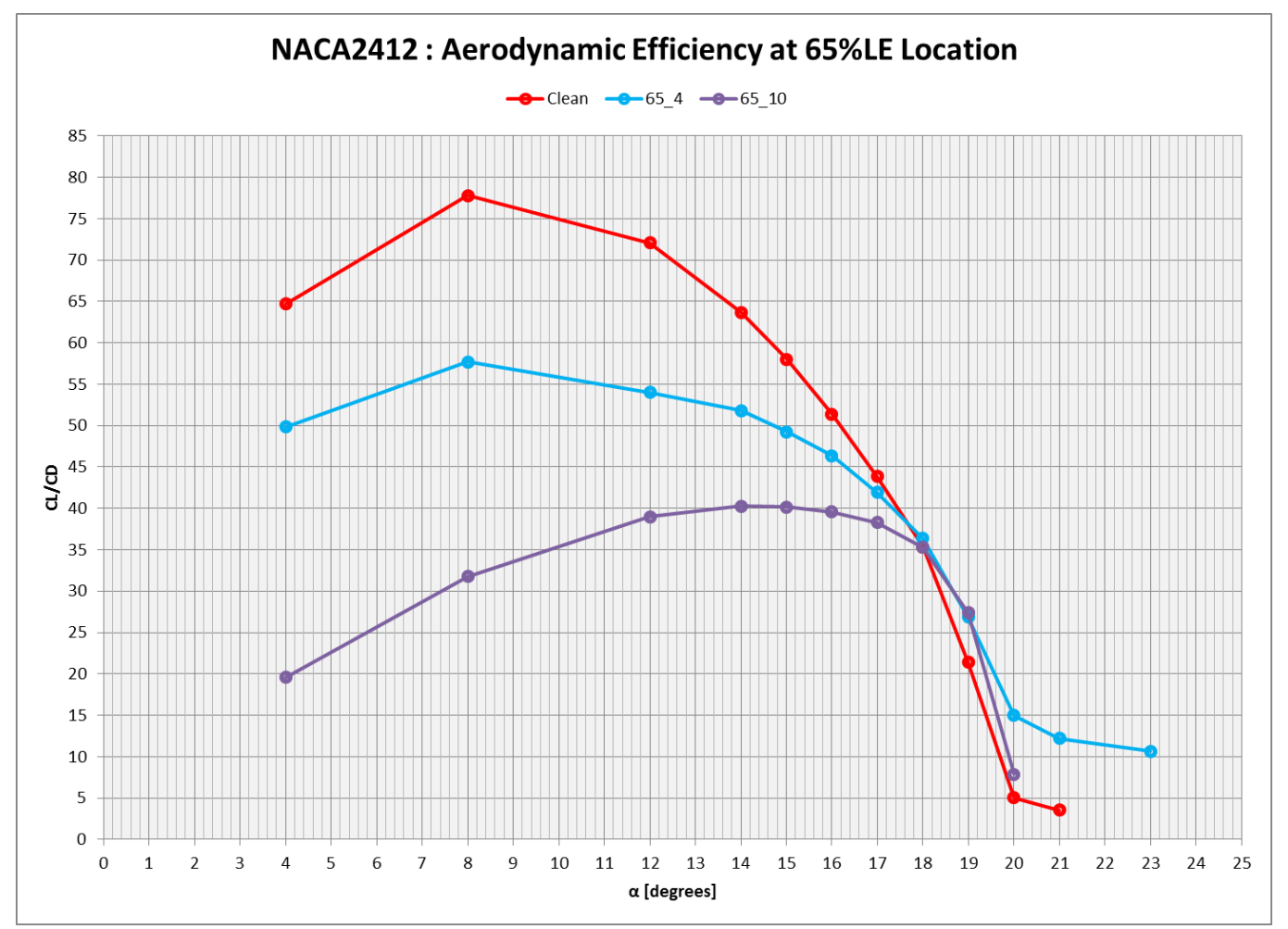

Figure 54: NACA 2412 Spoiler Study, CL/CD, 65\% LE location

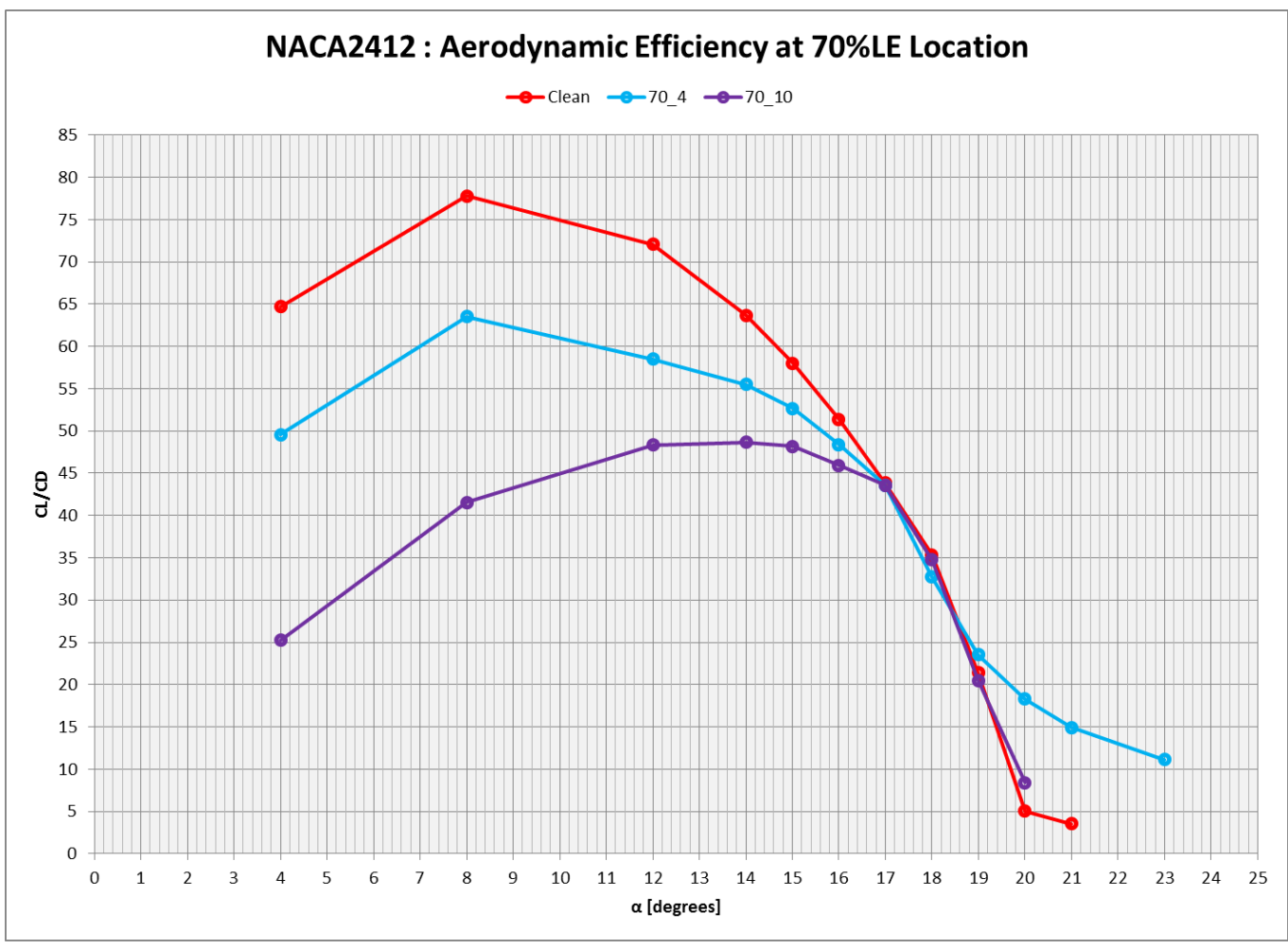

Figure 55: NACA 2412 Spoiler Study, CL/CD, 70\% LE location 
According to the results in Figure 53, Figure 54, and Figure 55 there was a gain in efficiency seen past 19 degrees for a spoiler deflection of 4 degrees in comparison to the baseline. Specifically taking the case of 19 degree alpha, the baseline efficiency was recorded to be approximately $21 \%$, and by adding the spoiler geometry at the $60 \% \mathrm{LE}$ location with a 4 degree deflection, improved the efficiency by an additional 7\%. Cross comparison at 65 and $70 \%$ location for the 19 degree alpha case, had shown an improvement of 6\% and 2\%. Interestingly, the results for 19 alpha had not only shown lift improvement, but analysis of the efficiency had provided additional insight that for each independent alpha case tested there was an ideal location for maximum efficiency gain for the particular case under consideration. For example, taking the 19 alpha case thus mentioned, the ideal location for maximum gain in efficiency was at the $60 \%$ leading edge location (Figure 53). However, although the efficiency at $70 \%$ was less than that seen at $60 \%$ leading edge location, the overall lift gain was discovered to be maximum at the $70 \%$ LE location. There was a $10 \%$ increase in lift seen at $70 \%$ in comparison to the baseline and the table below (Table 7) summarizes the numerical results seen for this case (alpha of 19 degrees and spoiler deflection of 4 in Figure 53, Figure 54 and Figure 55).

Table 7: Spoiler Study Results for alpha of 19 degrees, Transition SST model

\begin{tabular}{|lllll|}
\hline Mesh & Lift Coefficient & Drag Coefficient & \% Eff $(\mathrm{Cl} / \mathrm{Cd})$ & \% Lift Gain \\
\hline Baseline & 1.702 & 0.079 & 21.37 & - \\
\hline $\mathbf{6 0 \%}$ LE & 1.756 & 0.062 & 28.28 & $5.4 \%$ \\
\hline $\mathbf{6 5 \%}$ LE & 1.780 & 0.066 & 27.36 & $7.8 \%$ \\
\hline $\mathbf{7 0 \%}$ LE & 1.802 & 0.076 & 23.51 & $10 \%$ \\
\hline
\end{tabular}

Therefore, according to Table 7 along with an increase in lift coefficient there was an increase in drag coefficient moving from $60 \%$ to $70 \%$ leading edge location, which explains the results seen for aerodynamic efficiency.

In comparison, the results collected for the spoiler deflected at 10 degrees at 60\%LE (Figure 50) had shown a stall point at 19 degrees, that is two degrees past the baseline stall point. Similarly, this stall point remained constant for 65 and $70 \%$ leading edge location. However, although the stall point remained the same, the overall lift gain was seen to increase at this point moving further aft along the upper surface. This finding shows the overall increase in lift coefficient 
across all alphas tested from moving from 60\%-70\%LE location as seen in the displacement between each point and the baseline in the lift-alpha curves. For example, the point prior to stall shown on the spoiler curves for 4 and 10 degrees seen in Figure 50 at 60\%LE are further apart from their baseline points in comparison to those seen in Figure 52 at 70\%LE. Also, Figure 52, past stall shows an increasing lift curve that appears to be continuing past 21 degrees. Therefore, overall positive lift gain was seen across all locations tested with a 4 and 10 degree deflection. The aerodynamic efficiency at each alpha case tested for the spoiler at 10 degrees was overall seen to be less than that recorded for a deflected spoiler of 4 degrees. Therefore, along with finding that a prime location was seen for each alpha tested for maximum lift or efficiency, there was also an ideal spoiler deflection angle seen at a particular location tested. For example, an alpha of 19 degrees had shown maximum lift gain at 70\% LE of $10 \%$ for a spoiler deflection of 4 degrees in comparison to a spoiler at 10 degree deflection (5.7\% increase) at the same upper surface location.

To further delve into understanding the results seen in Figure 50-Figure 55, aerodynamic plots for the pressure coefficient, wall shear stress, wall y plus and streamlines were analyzed. Results were sectioned off between pre and post stall regions represented by the baseline study. For example, an alpha value at 16 degrees was seen to be a pre-stall point on the baseline, therefore, plots from the baseline as well as the 4 and 10 degree spoiler deflections were extracted from ANSYS Fluent to determine how the shape of the separation bubble, the separation point location, lift gain and as a check to see if the $y+$ was within suitable range. These plots were then compared to 17 degrees (the baseline stall point) in an effort to determine how the separation bubble changed, its location as well as to numerically determine where the majority of the increase in lift with the spoiler study was attained. This process was then repeated for alpha cases past stall, taken as 18 and 19 degrees as reference cases. Note: results for alpha cases past 19 degrees can be seen in the Appendix C.

\subsection{Pre-Stall Behaviour}

According to numerical plots collected for an alpha of 16 degrees (Please refer to Figure 50, Figure 51 and Figure 52) the lift coefficient was maximum at its baseline value of 1.702. The addition of the spoiler in fact hindered the lift coefficient which reduced from 1.702 at the 
baseline to 1.53 at $60 \% \mathrm{LE}$ for a spoiler deflection of 10 degrees; representing the furthest forward spoiler position and maximum spoiler deflection point. Therefore, for this pre-stall case, the spoiler behaviour was consistent with its original design function; that is to spoil the flow upon deployment. A clear indication that the baseline case was less affected by separation effects was a visual look of the streamlines. The deep blue coloured streamlines represent flow particles that are very near to zero velocity and therefore, represent a region of reduced flow, i.e the separation zone. Figure $56 \&$ Figure 57 show the separation bubble across all three locations, including the baseline for the 4 and 10 degree spoiler deflection angles. As shown in the figures seen (Figure 56 \& Figure 57), comparison of the results seen at the $60 \%$ location with the baseline, one can clearly see that for the baseline case the separation bubble is located much further aft of the spoiler location, and incorporation of the spoiler to the geometry forces this bubble to expand. Therefore, although the spoiler effectively retains the separation bubble, it had inadvertently expanded the separation bubble, thereby contributing to a loss of lift coefficient as these regions that normally were seen to have attached flow in the baseline case, were now showing areas of separated flow. This observation is further understood from a structural point of view, as the spoiler structure itself acts as a barrier, which forces upstream flow particles to deflect to the angle set by the spoiler deflection, which produces a change in the separation bubble to expand in area as well, as seen across all location in Figure 57. The following were the extracted streamlines seen for spoiler deflections of 4 and 10 degree 

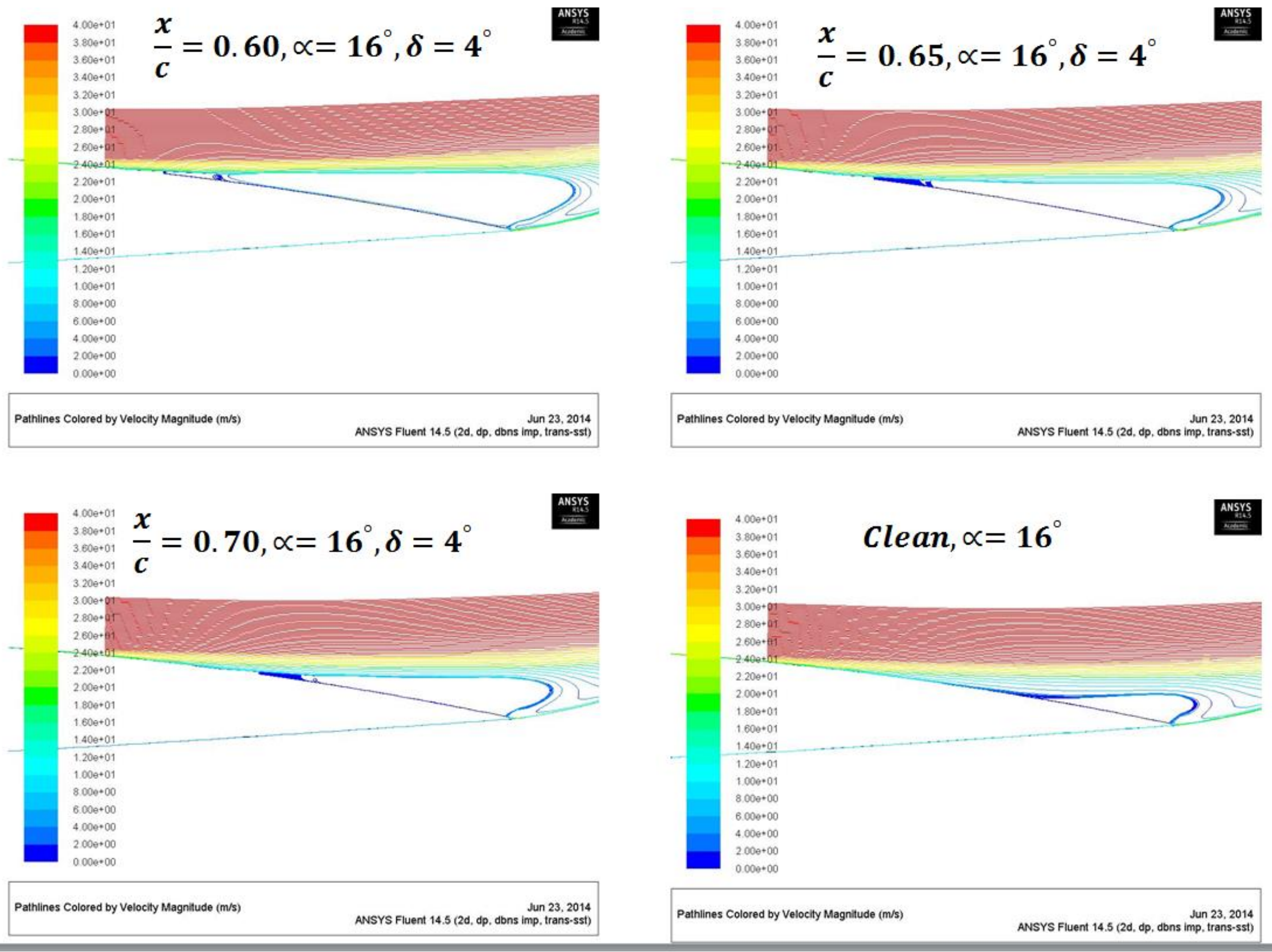

Figure 56: Velocity streamlines 60\%,65\% 70\% LE, Alpha=16, Spoiler deflection=4, M=0.17, Transition SST, Re=3.79E5 

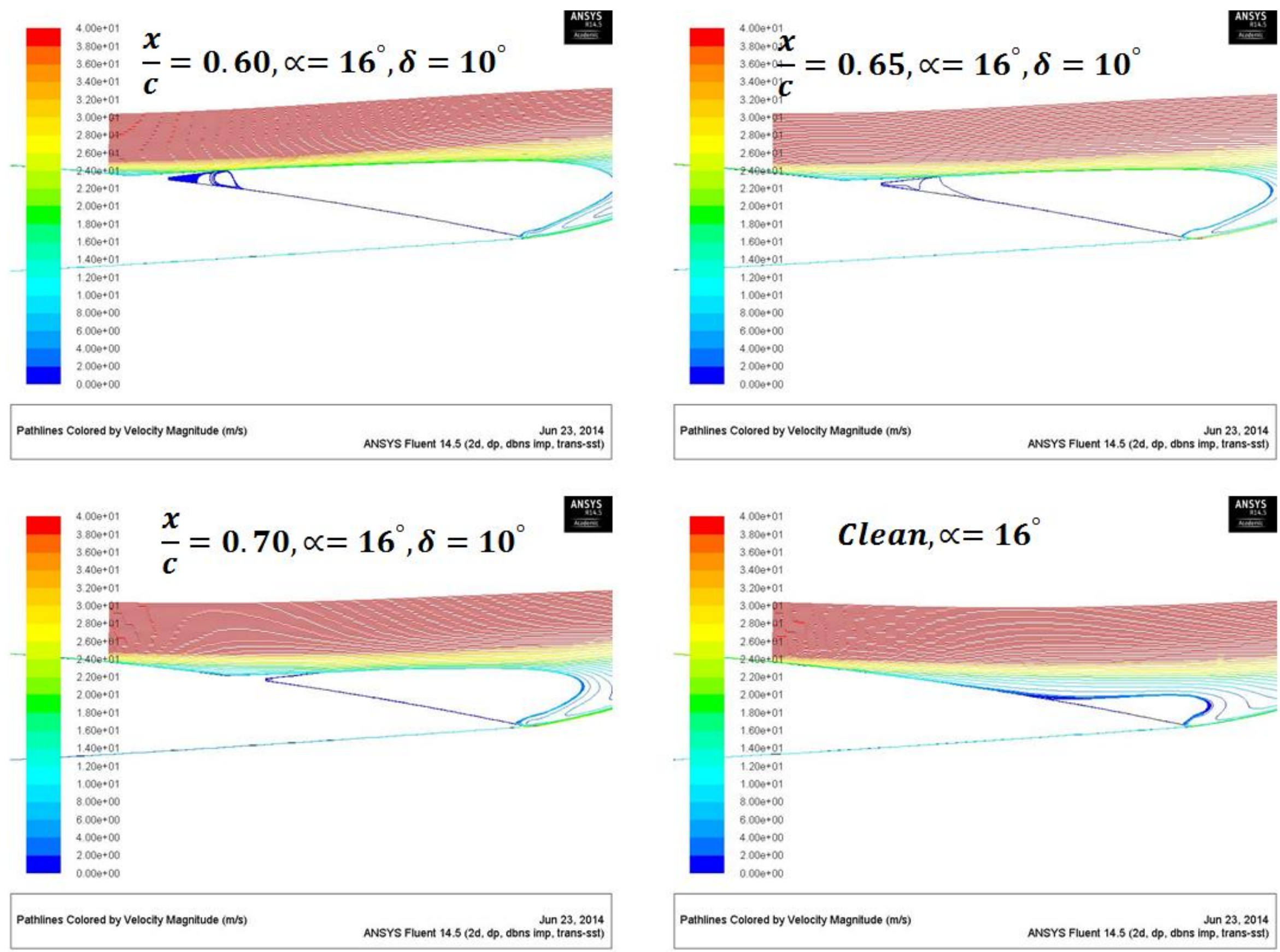
The pressure coefficient plots for a spoiler deflection of 4 and 10 degrees are shown in Figure 59 and Figure 58 on the next page. The red curve represents the results for the baseline case, and examining the upper surface, one can observe that the majority of any increase in lift production was governed by the greater area near the leading edge. According to Figure 59 the baseline curve shows a larger overall area between the upper and lower surfaces. The increase in area for the baseline is attributed to points located not only upstream of the $60 \%$ chord location but also between $60-80 \%$ LE. The pressure coefficient points for these two regions are at a higher value in comparison to the spoiler results. As mentioned earlier, the region between $60-70 \%$ is where the spoiler geometry is located, and a view of the baseline results here clearly shows that there is a lower pressure system located there for the baseline case that aids in lift production, therefore, the addition of the spoiler geometry removes and prevents this lower pressure region from forming. Also, the spoiler forward base location appears to govern the overall slope of the leading edge pressure coefficient curve. The black curve seen in Figure 59 and Figure 58 represents the 60\%LE location, which shows the least static pressure formation in area ahead of the $60 \%$ LE point, in comparison to the spoiler situated at the $70 \%$ location. The lower surface curves for all three locations including the baseline case were shown to have marginal variation. Notice, that the area under the baseline curve in Figure 59 and Figure 58 is largest upon comparison to the spoiler cases; an indication of an increased lift coefficient. Also note the leading edge change in pressure coefficient prior to the spoiler attachment point; here it is clearly seen that the spoiler leading edge base has an effect in the overall maximum leading edge $\mathrm{cp}$ production. The spoiler acts as a physical barrier preventing flow attachment upstream of its location. 


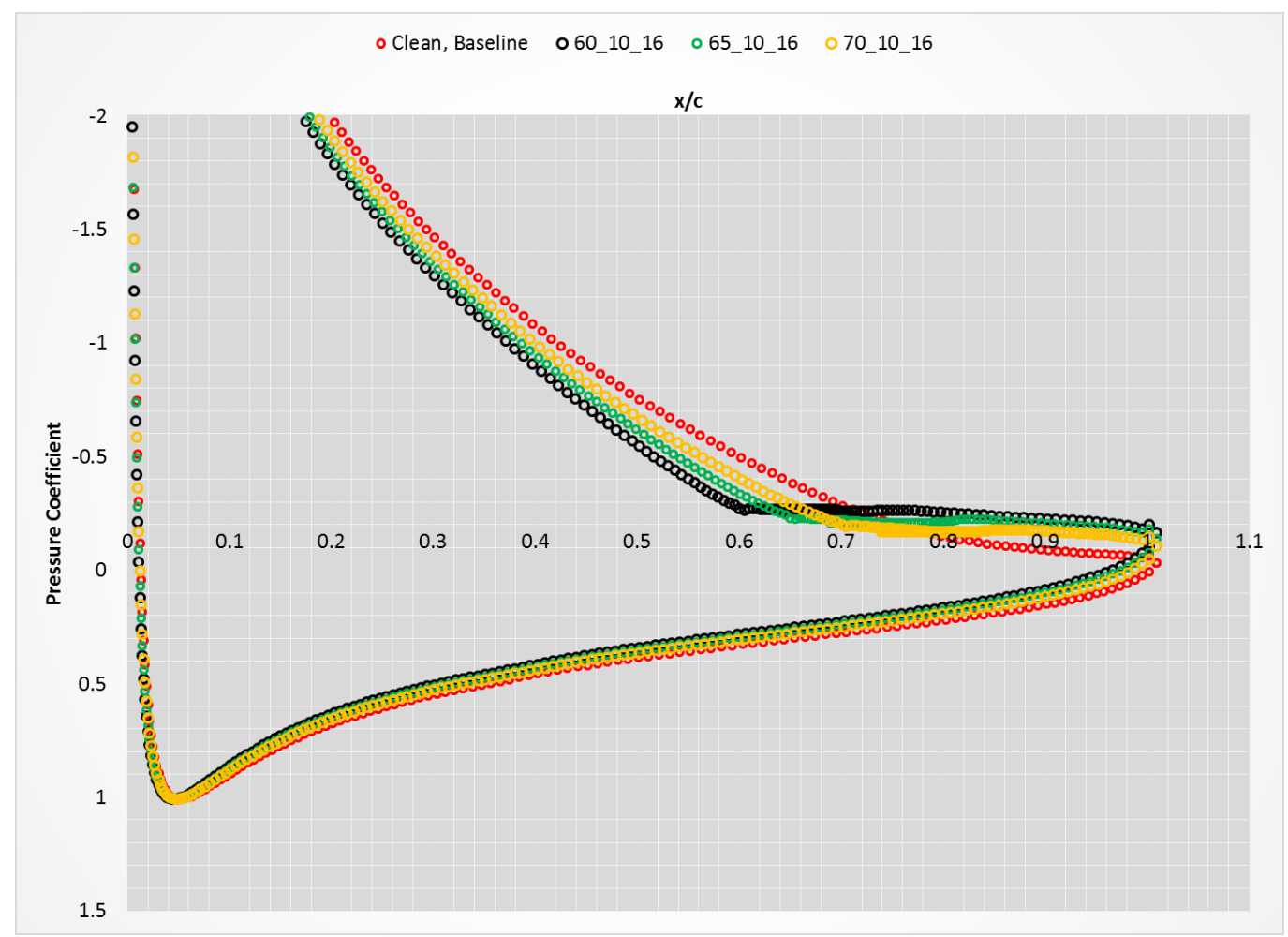

Figure 59: NACA2412 Spoiler Study, Pressure Coefficient, Spoiler deflection= 10 degrees, Alpha=16 degrees, Re=3.79E6, Transition SST Model

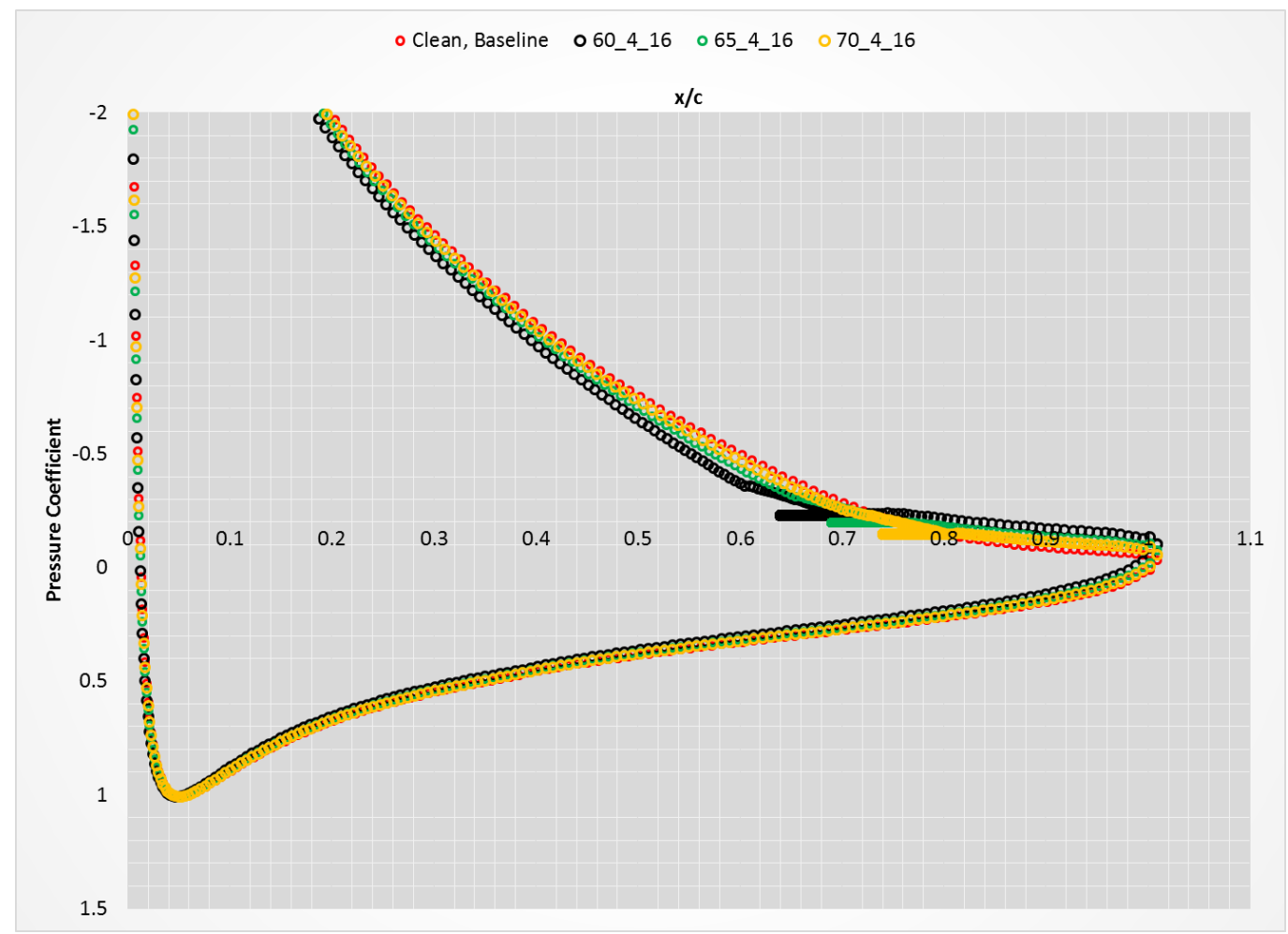

Figure 58: NACA 2412 Spoiler Study, Pressure Coefficient, Spoiler deflection = 4 degrees, Alpha=16 degrees, Transition SST Model, Re=3.79E6 
The wall shear stress plots for both the 4 and 10 degrees spoiler deflection cases at an alpha of 16 degrees are shown in the following figures:

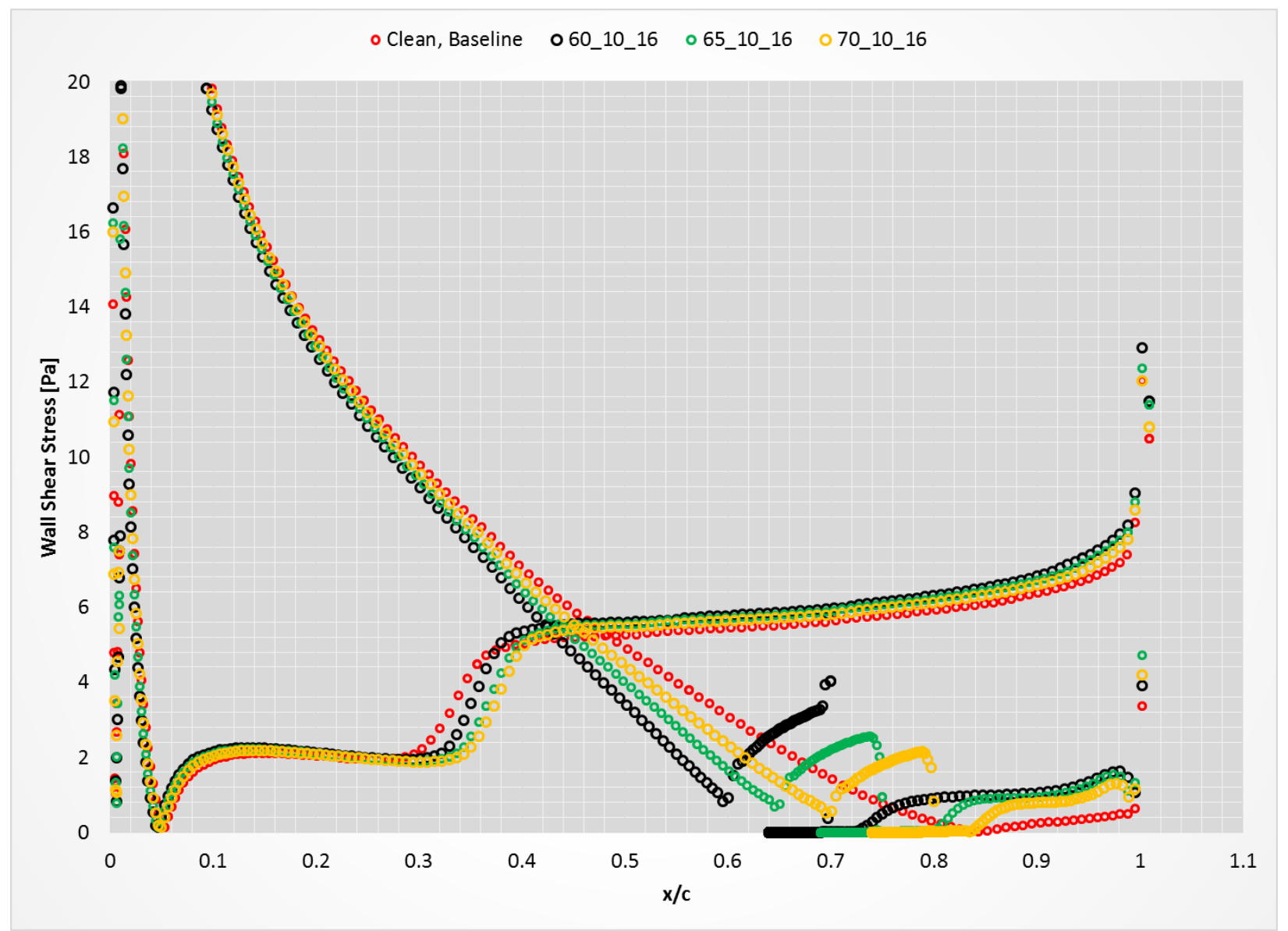

Figure 60: NACA 2412 Spoiler Study, Wall Shear Stress, Spoiler Deflection = 10 degrees, Alpha =16 degrees, Transition SST Model, Re=3.78E6 


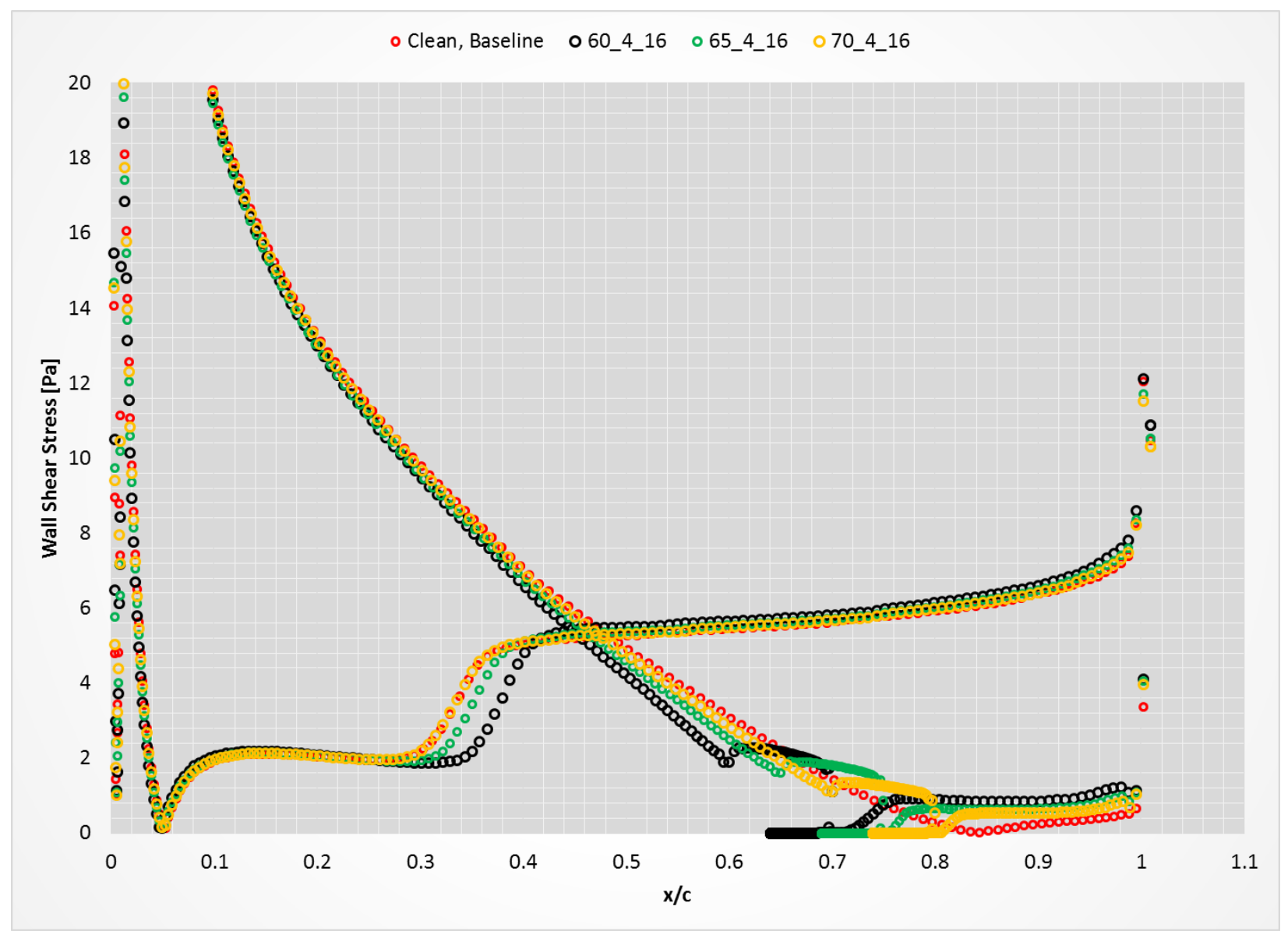

Figure 61: NACA 2412 Spoiler Study, Wall Shear Stress, Spoiler Deflection = 4 degrees, Alpha = 16 degrees, Transition SST Model, Re=3.79E6 
The wall shear stress plots shown in Figure 60 and Figure 61 (on the previous pages) provide details on the location of the separation point before the spoiler tip. The separation point for the baseline case is shown to be between 0.8-0.85 upper surface chord locations, whereas for both spoiler deflection cases (4 and 10 degree deflection) there appears to be no separation point on the airfoil surface itself. The points that do show a shear stress of zero on the plots referring to the spoiler curves are due to the presence of the spoiler structure itself. The wall y plus plots below are included for verification that the first node off the wall surface is within the acceptable range of order 1 for the SST turbulence model, which is clearly the case at most points. Please see Appendix $\mathrm{C}$ for further details on the Wall y plus plots.

For the remainder of the analysis, results for the 10 degrees spoiler deflection can be found in Appendix C. As described in the previous section for an alpha of 16 degrees alpha, post analysis of the 10 degrees spoiler deflection provided less effective performance than the clean airfoil and to what was observed for the 4 degree spoiler deflection case. Therefore, the remainder of the sections will focus on spoiler location on the upper surface with airfoil pitch angle for a deflected spoiler of 4 degrees. Along with the remainder of the 10 degree spoiler deflection, wall Y+ plots for remainder of the cases can also be found in Appendix C, as these plots serve to show that the results are within the viscous region required to extract meaningful results involving boundary layer separation.

\subsection{Post-Stall Behaviour}

Streamlines coloured in velocity magnitude were extracted for airfoil attack angles of 17-19 degrees (alpha angles) shown in Figure 62 Figure 63, ad Figure 64. Note that since the transient separation bubble will oscillate in size, the image of the streamlines (along with the remainder of the results for pressure coefficient, and wall shear stress) is by no means rigorously used for comparison. As described for the alpha case of 16 degrees, the main objective of extracting such a plot were to visually see how the flow responds with inclusion of the spoiler geometry in comparison to that seen for the baseline, clean case. The streamlines were utilized to see how the separation bubble changed as the pitch angle was increased. For ease of reference the streamline plots for pitch angles of 17-19 were included sequentially below for the viewer to see the changes in the separation bubble with location for the spoiler deflection of 4 degrees. 
Upon examination of Figure 62-Figure 64 it becomes clearly apparent that the separation bubble for the baseline case as the pitch angle moves from 17-19 degrees grows in area due to an intensifying adverse pressure gradient. The overall shape of the separation bubble provides information on whether the spoiler will improve the lift or reduce it. For example, upon examination of the baseline separation bubble for an alpha of 17 degrees, one can see the bubble is located further downstream from any of the spoiler locations. Specifically, the wall shear stress plot shown in Figure 65 shows the separation point is approximately at 0.75 chord location; aft of the farthest spoiler location tested at $70 \%$ leading edge. For the spoiler to effectively retard stall, ideally the spoiler should be placed in the zone of the flow separation so as to act as a barrier and effectively capture the separation bubble downstream and in return improve the lift. Visual observation of the streamlines for 17 degrees alpha in Figure 62, shows the separation bubble was seen to have expanded at the $60 \%$ leading edge location in comparison to the clean, baseline result; similar to what was seen for an alpha of 16 degrees. This causes a reduction in the overall lift coefficient as a result of a loss in pressure recovery that has now been replaced by the separated flow. The key transition to when the spoiler begins to retard stall was seen in the streamlines of 18 degrees in Figure 63. Here the separation point for the baseline case was seen to occur at around 65\% leading edge location, as shown in Figure 66. Post analysis of the streamlines for the spoiler at $65 \%$ leading edge location with a deflection of 4 degrees shows a separation bubble comparable to the baseline, and according to results, the aerodynamic efficiency here was seen to increase by almost $1 \%$ when compared to the baseline. A key indication of lift improvement is provided by the pressure coefficient plots. Figure 70 clearly shows the absolute area between the upper and lower surfaces for the $65 \%$ (green curve) and $70 \%$ (yellow curve) is much larger than the baseline (red curve). Similarly, these observations are seen for an alpha of 19 degrees, which had shown lift improvement across all three spoiler locations, as shown in Figure 69. The total area between the upper and lower surfaces for pressure coefficient had shown an enlarged region for $60 \%, 65 \%$ and $70 \%$ spoiler location in comparison to the baseline. According to the numerical results shown in Figure 50, Figure 51 and Figure 52 the lift coefficient for an alpha of 19 degrees was found to be around 1.702, with the inclusion of the spoiler the lift rose to $1.75,1.78$ and 1.80 at $70 \%$ leading edge location. Overall, the lift production at 60 and $65 \%$ leading edge locations was found to be comparable; however there is a distinct increase seen at $70 \%$ when analyzing the plot shown in Figure 69. A look at the 
wall shear stress plot further validates this observation. Figure 67 shows the wall shear stress plot for an alpha of 19 degrees, for both the baseline and spoiler deflected at 4 degrees. According to the figure, the baseline case shows a separation point at about $0.55 \mathrm{x} / \mathrm{c}$ chord location. With the inclusion of the spoiler across all location tested (60-70\% leading edge location) it is difficult to spot a separation point as the spoiler geometry shows areas of zero shear stress in locations just underneath the spoiler tip (spoiler hinge cavity). Therefore, the spoiler was effective in mitigating stall for pitch angles whose baseline separation bubble was found to be near or forward of the upper surface spoiler locations tested (60-70\% leading edge chord locations) as the spoiler acted as a barrier, preventing the developing trailing edge separation bubble from moving forward. Results had shown positive lift gain for alpha angles of 18 degrees or more. Improvement was seen to push the stall point forward, along with the separation point location of the separation bubble. Results for pitch angles of 20-21 \& 23 degrees can be found in Appendix $\mathrm{C}$ section of this report. 

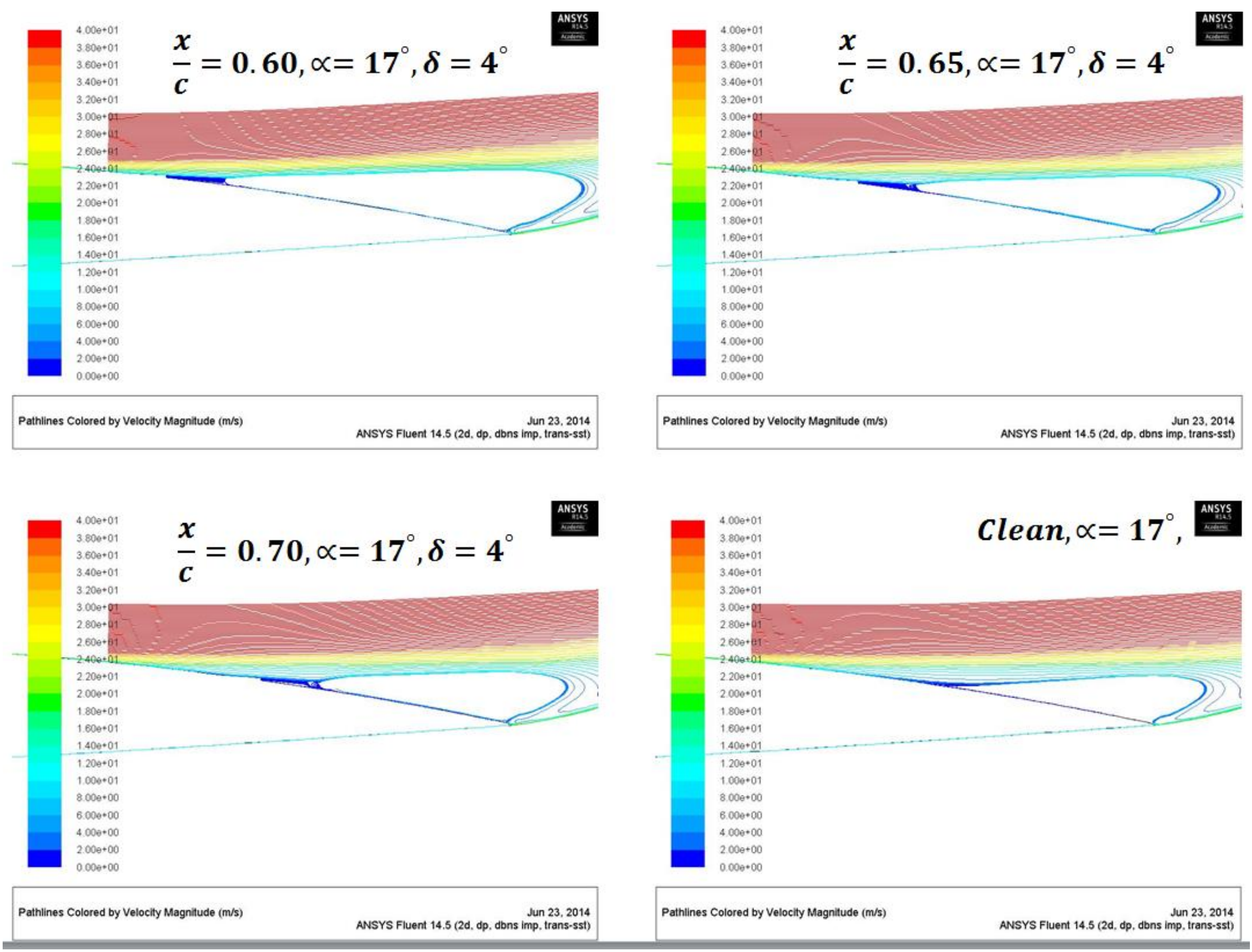

Figure 62: Velocity Magnitude Streamlines, Alpha=17 Degrees, Transistion SST Model, Re=3.79E6 

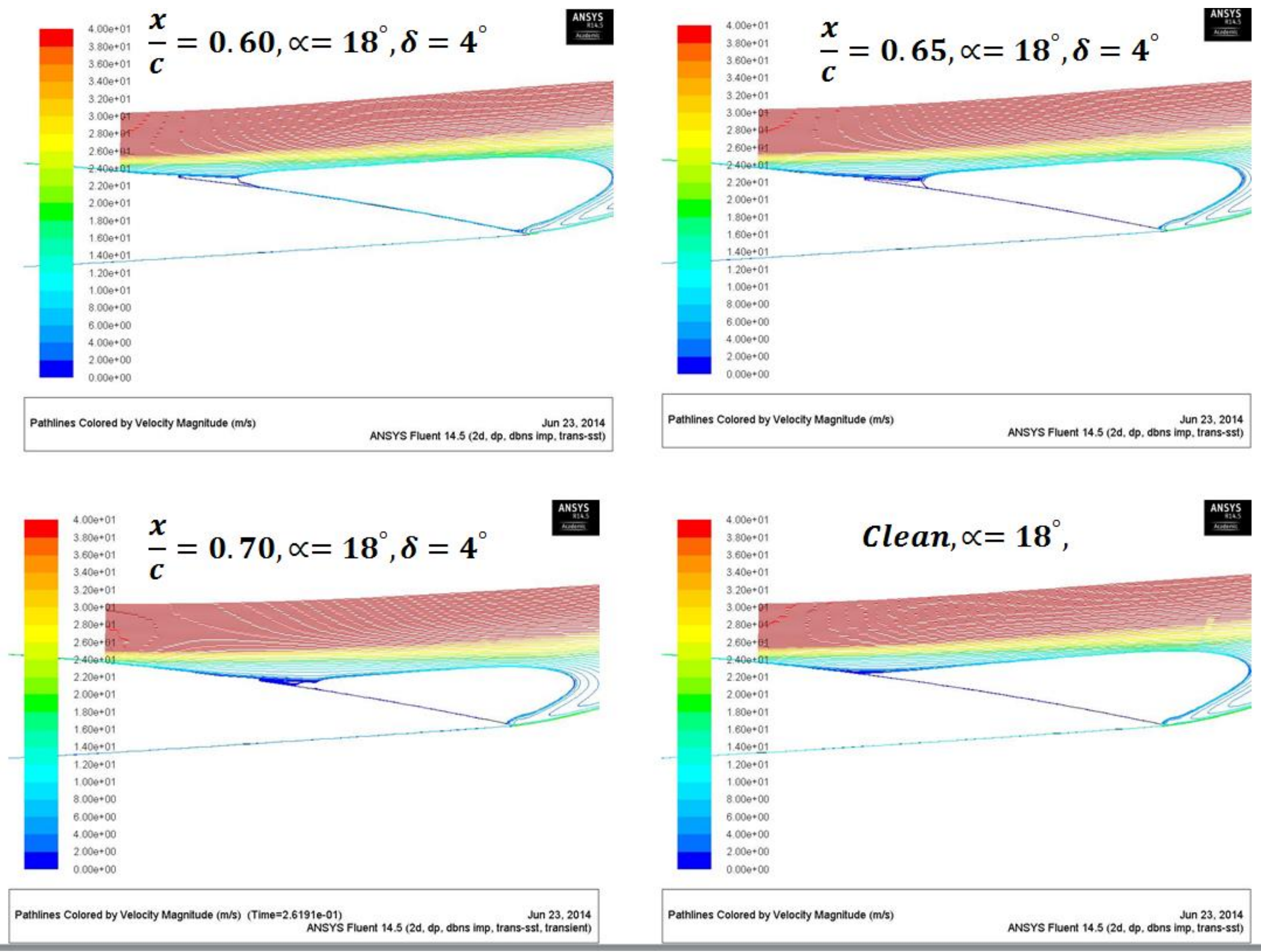

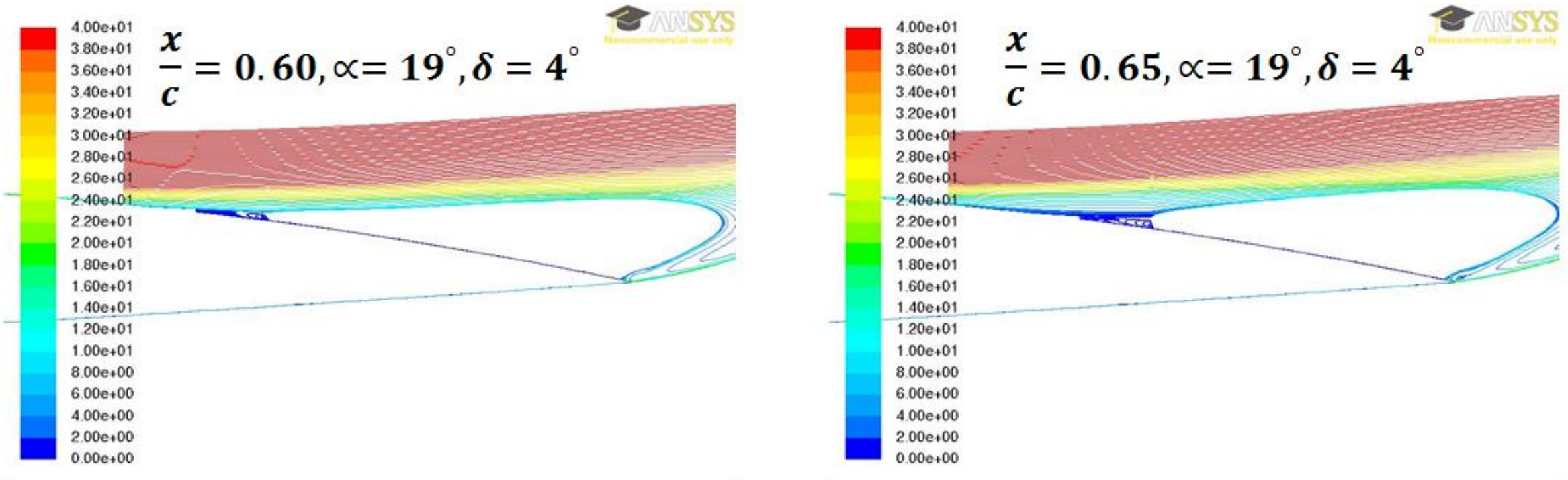

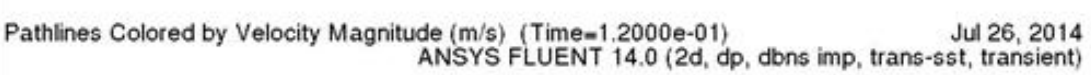

Pathlines Colored by Velocity Magnitude (m/s) (Time-2.0749e-01) May 14, 2014 ANSYS FLUENT 14.0 (2d, dp, dbns imp, trans-sst, transient)
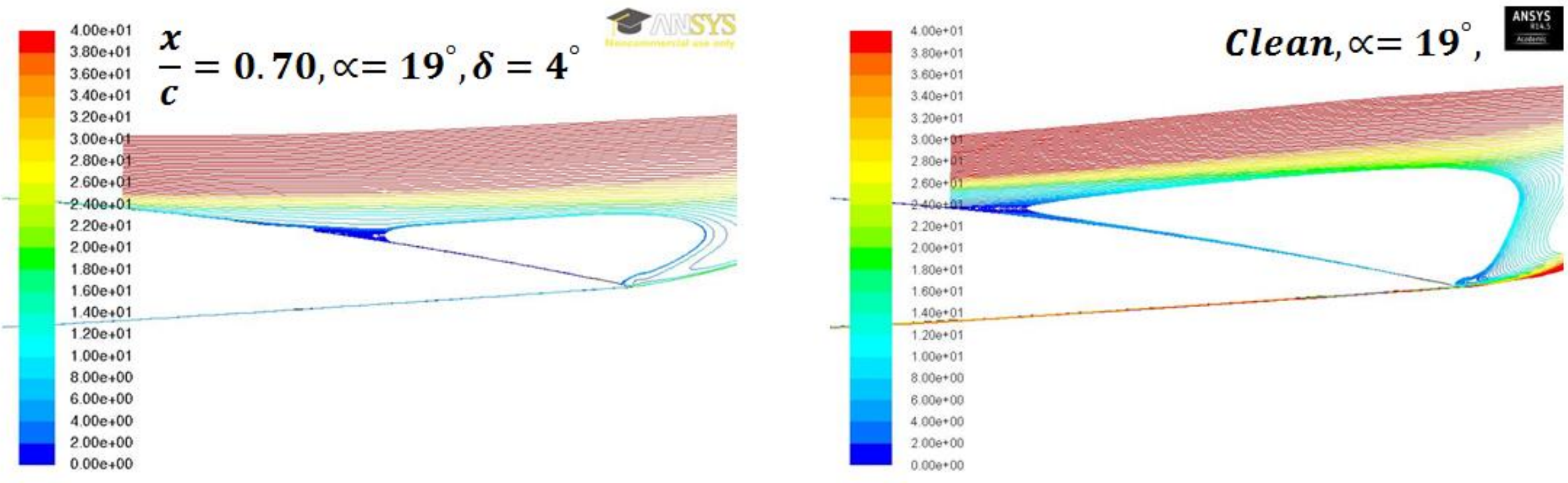

Pathlines Colored by Velocity Magnitude (m/s) (Time-1.3001e-01) May 21, 2014 ANSYS FLUENT 14.0 (2d, dp, dbns imp, trans-sst, transient)

Pathlines Colored by Velocily Magnitude $(\mathrm{m} / \mathrm{s})($ Time=3.1282e-01) May 29, 2014 ANSYS Fluent 14.5 (2d, dp, dbns imp, trans-sst, transiemt)

Figure 64: Velocity Magnitude Streamlines, Alpha=19 Degrees, Transition SST Model, Re=3.79E6 


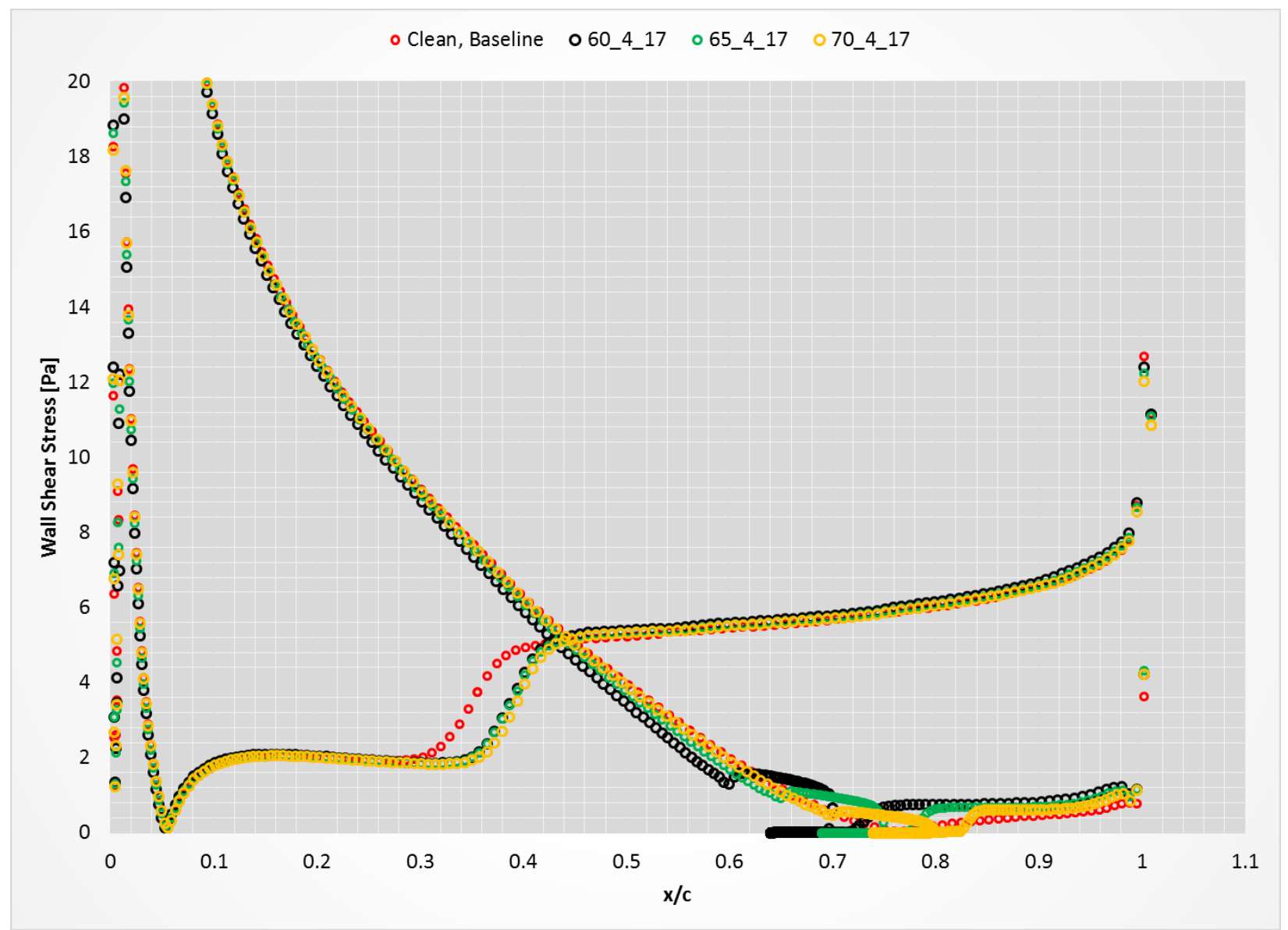

Figure 65: Wall Shear Stress, Alpha=17 Degrees, Spoiler Deflection $=4$ Degrees Transition SST Model, Re=3.79E6 


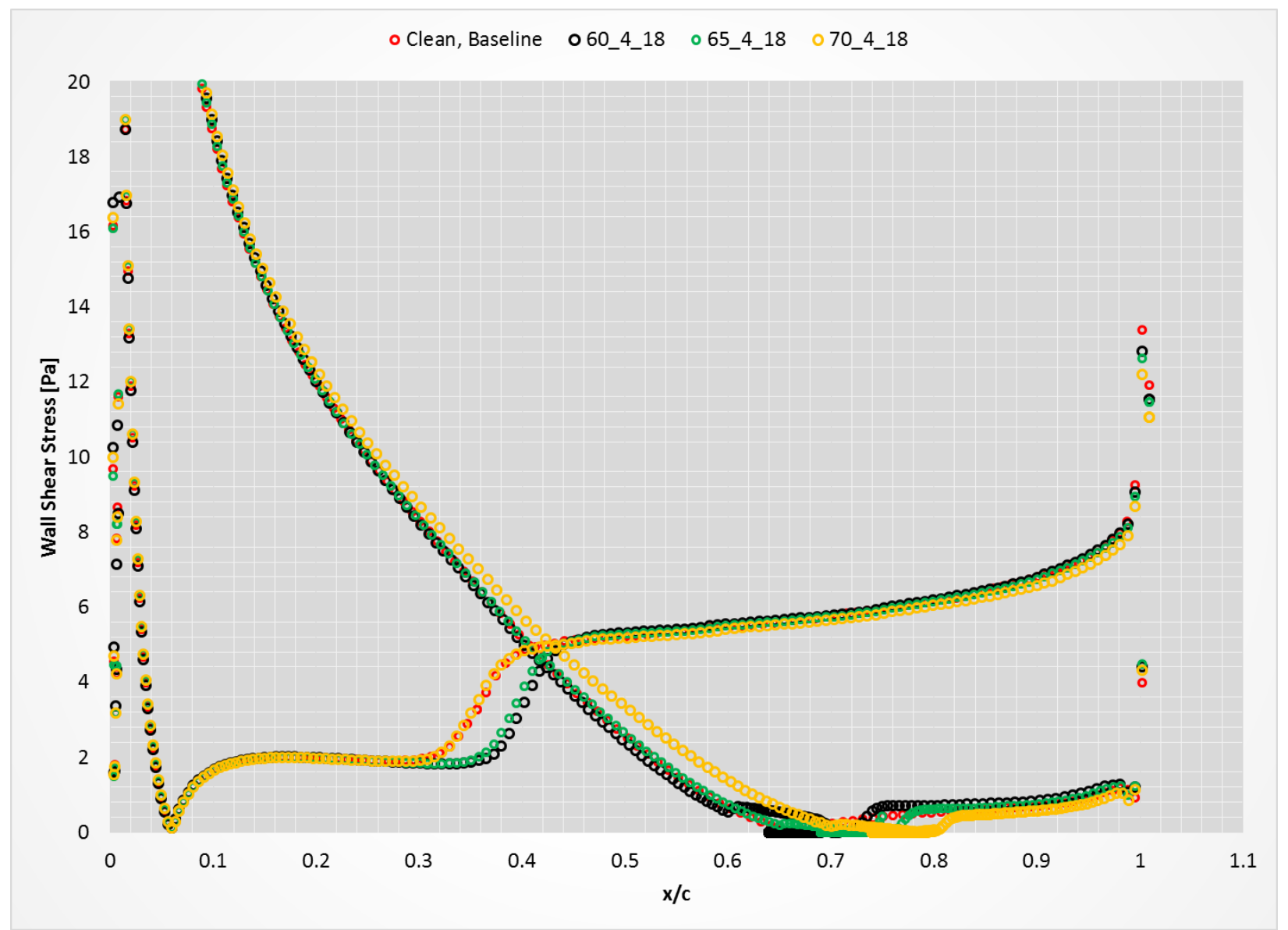

Figure 66: Wall Shear Stress, Alpha $=18$ Degrees, Spoiler Deflection $=4$ Degrees, Transition SST Model, Re $=3.79 E 6$ 


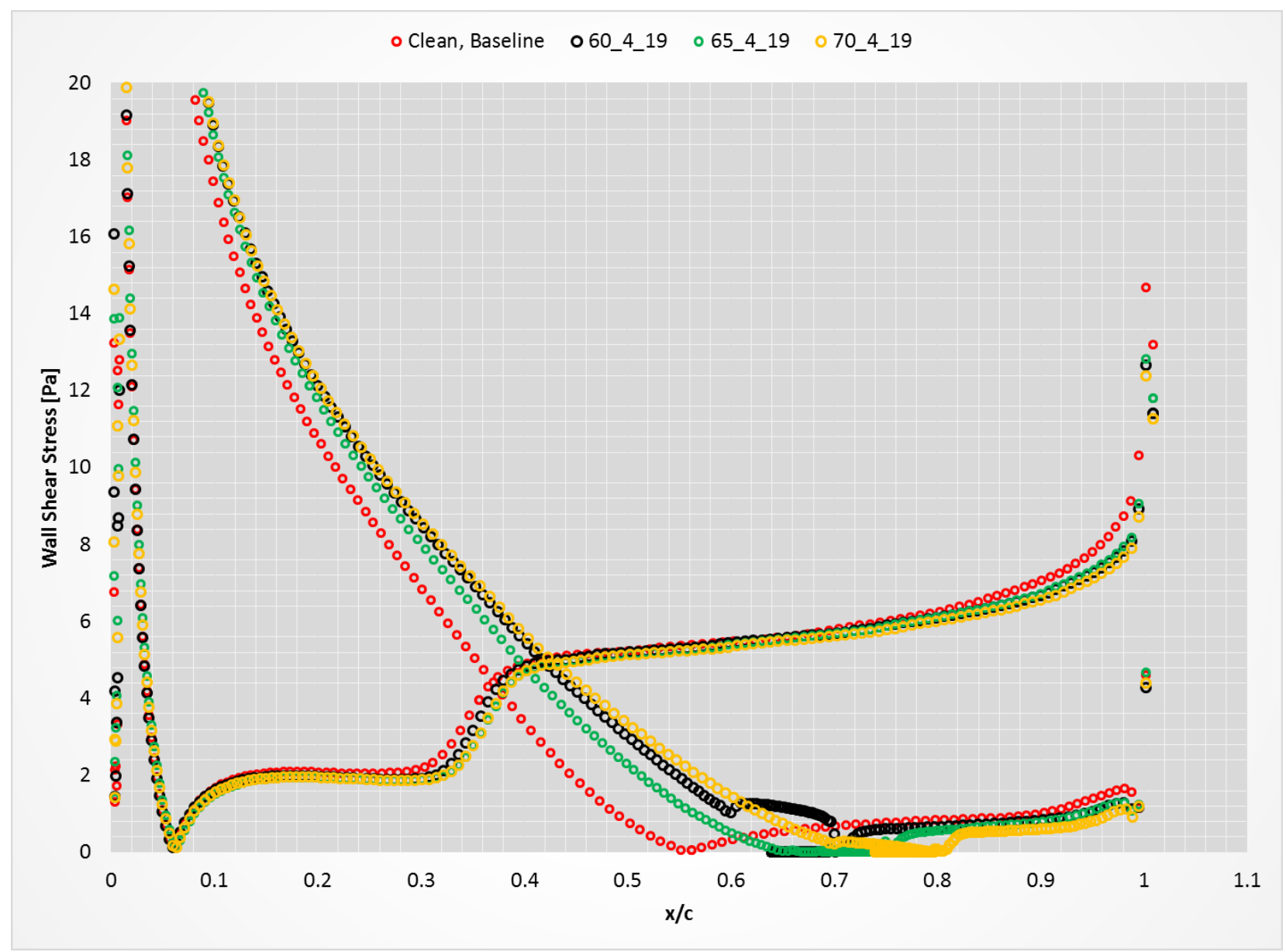

Figure 67: Wall Shear Stress, Alpha=19 Degrees, Spoiler Deflection = 4 Degrees, Transition SST Model, Re=3.79E6 


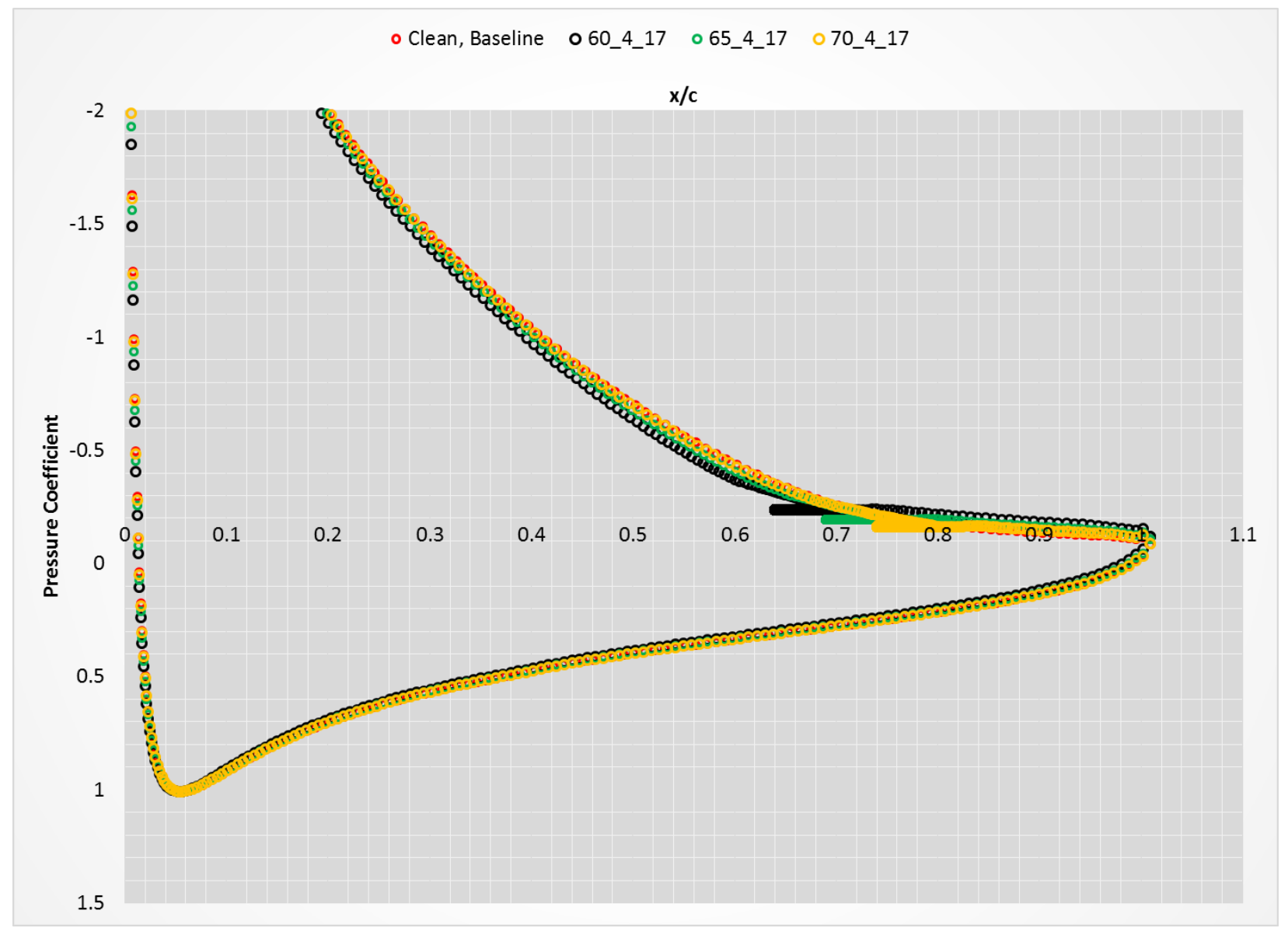

Figure 68: Pressure Coefficient, Alpha=17 Degrees, Spoiler Deflection= 4 Degrees, Transition SST Model, Re=3.79E6 


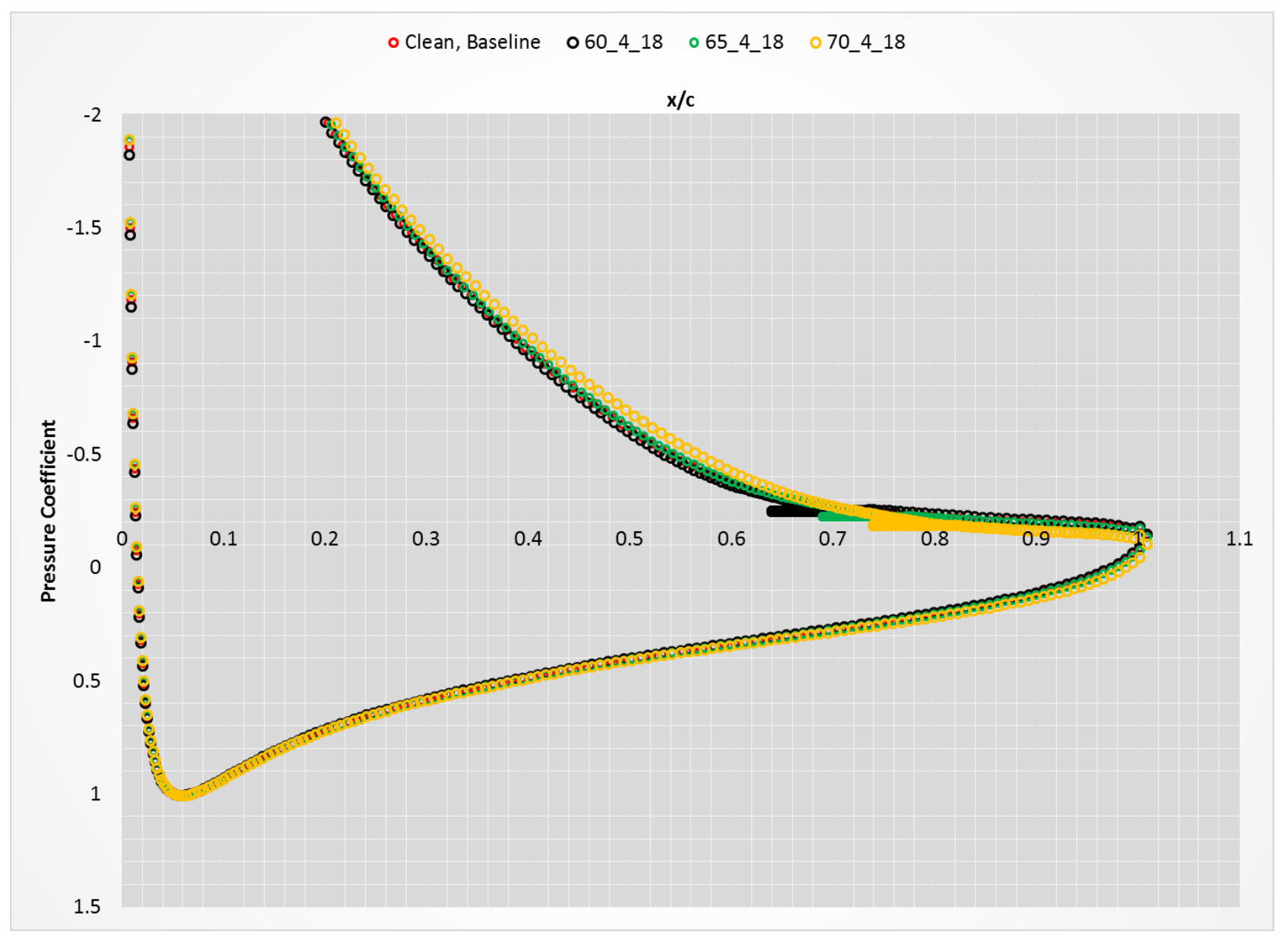

Figure 69: Pressure Coefficient, Alpha= 19 Degrees, Spoiler Deflection $=4$ Degrees, Transition SST Model, Re=3.79E6 


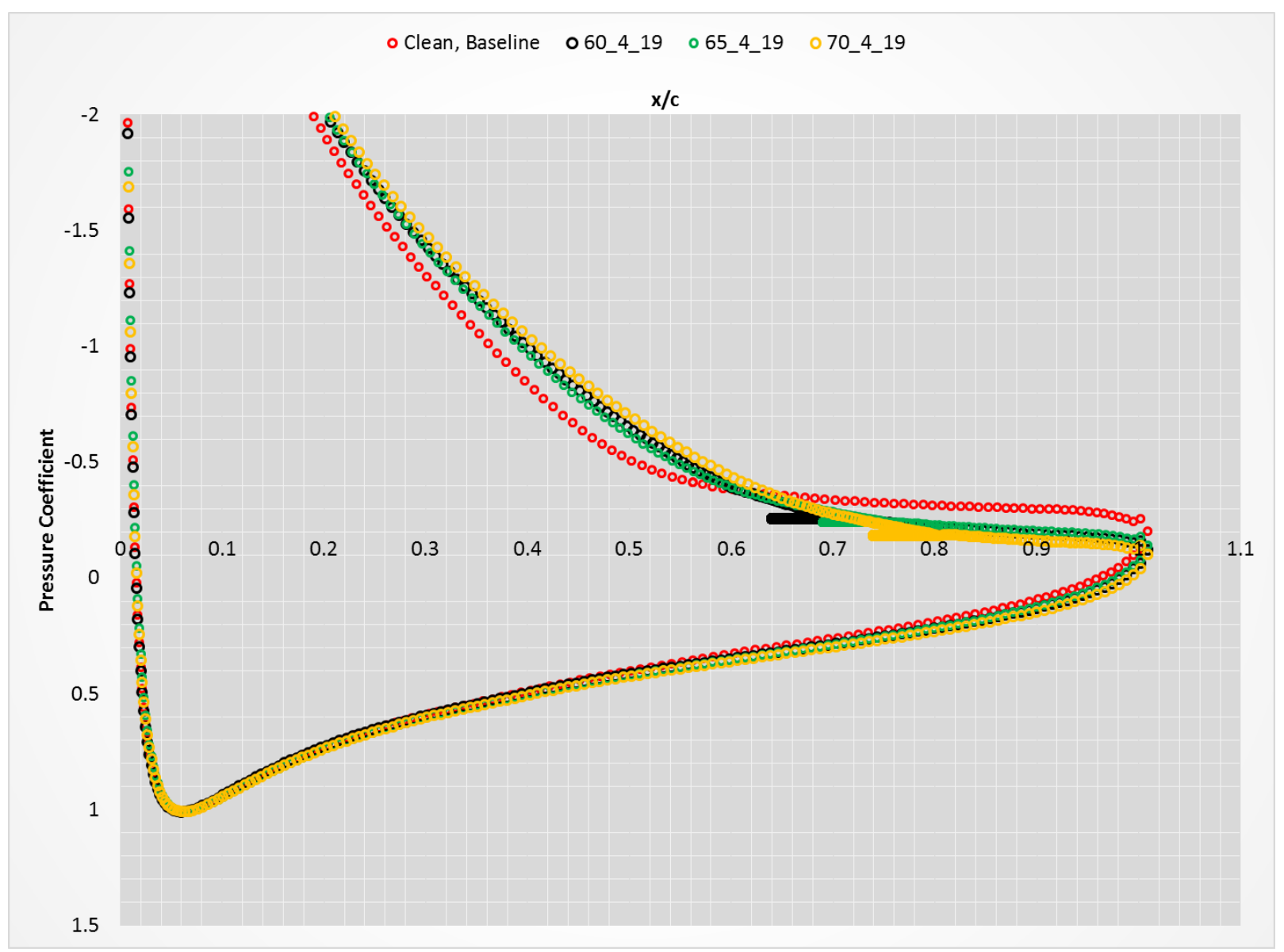

Figure 70: Pressure Coefficient, Alpha=18 Degrees, Spoiler Deflection= 4 Degrees, Transition SST Model, Re=3.79E6 


\section{Chapter 5}

\section{Conclusion}

The NACA 2412 airfoil section was studied for aerodynamic lift performance with the addition of a spoiler geometry connection. The NACA 2412 section had chord length of about 1 [m]. The spoiler size was taken as $10 \%$ chord length and aerodynamic performance of lift, drag, and overall wake characteristics were analyzed at 60\%, 65\% and 70\% leading edge chord locations. Specifically, velocity streamlines were utilized to assess the development of the separation bubble as the spoiler was deflected between low to high airfoil pitch angles. The spoiler deflection was increased to a point where the lift was seen to degrade with increasing pitch angles. According to results, a spoiler deflection of 10 degrees had started showing sign of decrease in lift in areas that had shown a positive increase for the 4 degree deflection point. Therefore, spoiler deflection angles of 4 and 10 degrees were analyzed across all upper surface locations tested (60\%-70\% leading edge). Overall the following were the results summarized:

- The lift was seen to increase past 18 degree angle of attack across all locations tested for a spoiler deflection of 4 degrees

- $60 \%$ LE location, 4 degree spoiler deflection the stall point was pushed past 21 degree alpha

- $65 \%$ \& $70 \%$ LE locations with the addition of the 4 degree spoiler deflection- the stall point was seen to be much greater than 23 degrees (maximum alpha tested to date)

- Baseline aerodynamic efficiency at 19 degrees saw a 7\% increase via 4 degree spoiler deflection at $60 \%$ LE location ( $6 \%, \& 2 \%$ increase seen at $65 \%$ and $70 \%$ LE locations)

- Separation point was analyzed via wall shear stress plots which had shown a separation point of $0.55 \mathrm{x} / \mathrm{c}$ for 19 degrees, baseline case; however, with the inclusion of the spoilerno clear separation point was detected, therefore, the spoiler was effective in maintaining attached flow and re-gaining the pressure loss that was found for the baseline case. 


\section{References}

1. Experimental Investigations of the Flowfield of an Airfoil with Spoiler. Chyang S. Lee, Satya Bodapati. No.11, Stanford, California : AIAA Journal, November 1987, Vol. 25.

2. Study of an Airfoil with a Flap and Spoiler. Abdullah Al Shabibi, Munir Al Hammadi and Joseph Mathew. No.6, Muscat, Oman : Journal of Aircraft, 1994, Vol. 31.

\section{Aircraft Spoiler Effects Under Wind Shear. M.M. Abdelrahman, M.A. Ghazi, I.A. Olwi and A.M. Al-}

Bahit. No.1, Jeddah Saudi Arabia : Journal of Aircraft, 1994, Vol. 31.

4. Computational Model for Low Speed Flows Past Airfoils with Spoilers. N.J. Pfeiffer, G.W. Zumwalt. No.3, Seattle, Washington, Wichta, Kan. : AIAA, 1981, Vol. 20.

5. Aerodynamic Characteristics of a Two-Dimensional Moving Spoiler in Subsonic and Transonic Flow. $\mathbf{H}$ Consigny, A. Gravelle, and R. Molinaro. NP. 9, s.I. : Journal of Aircraft, 1994, Vol. 21.

6. Unsteady Aerodynamic Characteristics of Airfoil with Moving Spoilers. Yeung, C. Xu and W.W.H. No. 3, Singapore : Journal of Aircraft, 1999, Vol. 36.

7. Reduction of Transient Adverse Effects of Spoilers. W.W.H Yeung, C. Xu, and W. Gu. No. 4, Singapore : Journal of Aircraft, 1997, Vol. 34.

8. Parameteric Study of Transient Spoiler Aerodynamics with Two Equation Turbulence Models. SeongWook Choi, Keun-Chik Chang, and Honam Ok. No.5, s.I. : Journal of Aircraft, 2001, Vol. 38.

9. A Method for Predicting Lift Effectiveness of Spoilers at Subsonic Speeds. Arthur L. Jones, Owen P. Lamb and Alfred E. Cronk. No. 4, New York : Journal of the Aeronatical Sciences, 1956, Vol. 23.

10. Investigation of a bio-inspired lift-enhancing effector on a 2D airfoil. Ashok Gopalarathnam, Joe Johnston. North Carolina : Bioinspiration \& Biomimetics, 2012, Vol. 7. 10.1088/1748-3812/7/3/036003.

\section{Experimental Investigation of Self-Actuating Upper-Surface, High Lift-Enhancing Effectors. Goetz} Bramesfeld, Mark D. Maughmer. No. 1, Pennsylvania State University, University Park, Pennsylvania : Journal of Aircraft, 2002, Vol. Vol. 39.

12. Bruce R Munson, Dennis J. Cronin. Aifroils/ Wings. [book auth.] Richard C. Dorf. The Engineering Handbook. s.I. : CRC Press LLC, 1998. 
13. E.J.Rogers, H.c. Seetharam, and W.H. Wentz, Jr. Experimental Studies of Flow Separation of Three Airfoils at Low Speeds. Wichita, Kansas : National Aeronautics and Space Administration, 1982.

14. Doenhoff, Ira H. Abbott and Albert E.Von. Theory of Wing Sections. New York : Dover Publications Inc., 1949. 486-60586-8.

15. Mr. Alex Tsoulis, Mr. Neal A. Harrison. Structures Director at EXMD Inc. and Boeing Employee (Boeing R\&D). Webex Meeting. Toronto, 102013.

16. Chattot, Jean-Jacques. Computational Aerodynamics and Fluid Dynamics. s.I. : Springer-Verlag Berlin Heidelberg , 2002. ISBN 3-540-43494-1.

17. M. Hallback, D.S. Henningson, A.V. Johansson and P.H Alfredsson. Turbulence and Transition Modelling. Stockholm : Kluwer Academic Publishers, 1995. 0-7923-4060-4.

18. H. Schlichting, K. Gersten. Boundary Layer Theory. s.I. : Springer, 2000. 3-540-66270-7.

19. Roache, Patrick J. Verification and Validation in Computational Science and Engineering. Albuquerqe, New Mexico : Hermosa Publishers, 198. 0-91348-08-3.

20. The Prediction of Laminarization with a Two Equation Model of Turbulence. W.P Jones, and B.E Launder. 1972, International Journal, Heat and Mass Transfer, pp. Vol 15, pp.301-314.

21. A method for Prediciting Lift Effectivness of Spoilers at Subsonic Speeds. Arthur L. Jones, Owen P. Lamb, and Alfred E. Cronk. No. 4, s.I. : Journal of te Aeronuatical Sciences, 1956, Vol. 23. 


\section{Appendix A: Baseline NACA 2412 Mesh Details}

The following is a layout of the Domain and Fluid sections described in Table 8 and Table 9 on page $89-90$ of this report. The lettered areas provide additional information in regards to the Blocking edge length and ratio's used for mesh generation. Details of these sections can be found on the following page.

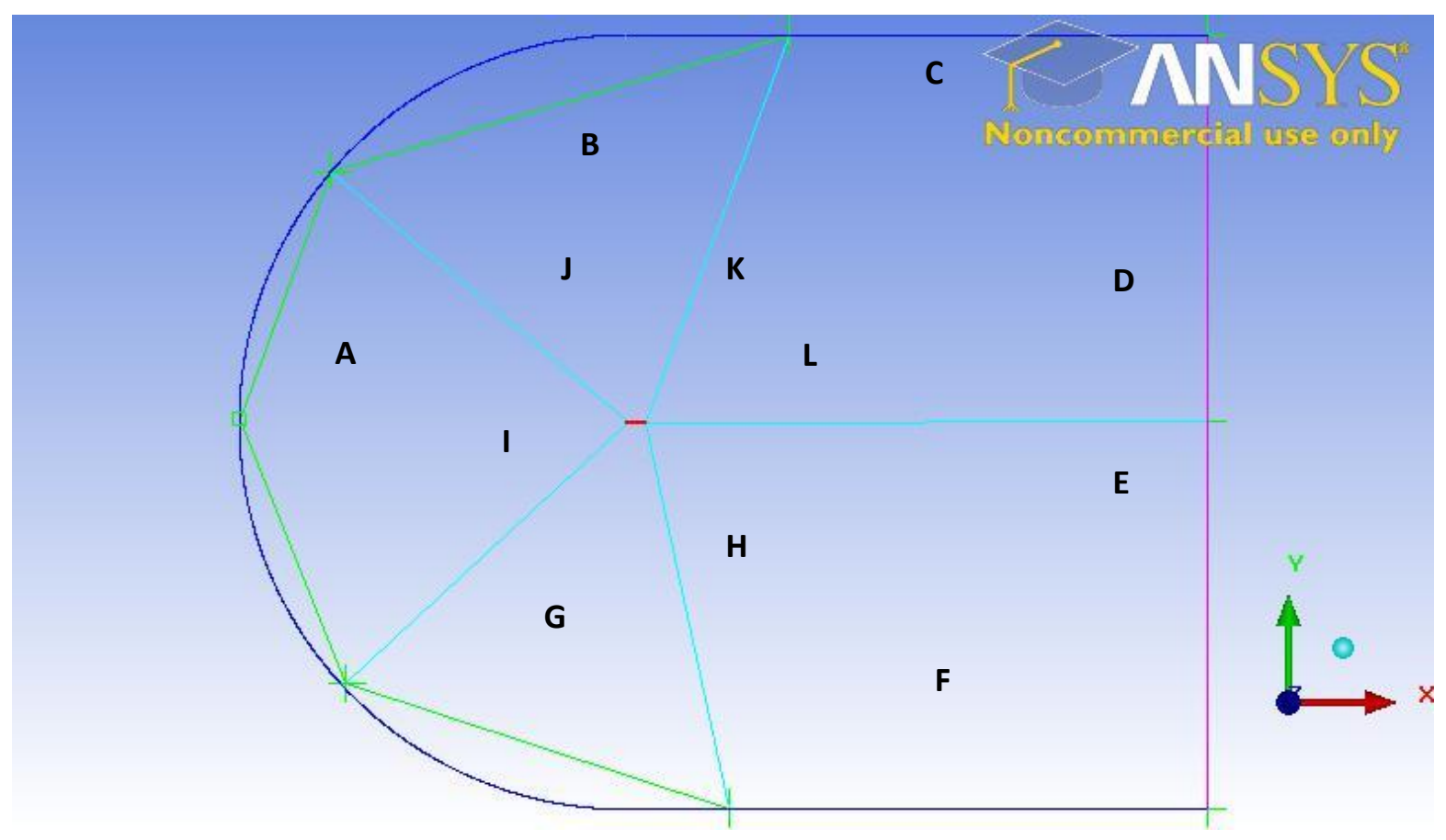

Figure 71: Mesh Layout of Domain and Fluid Region of Baseline NACA2412 airfoil section 


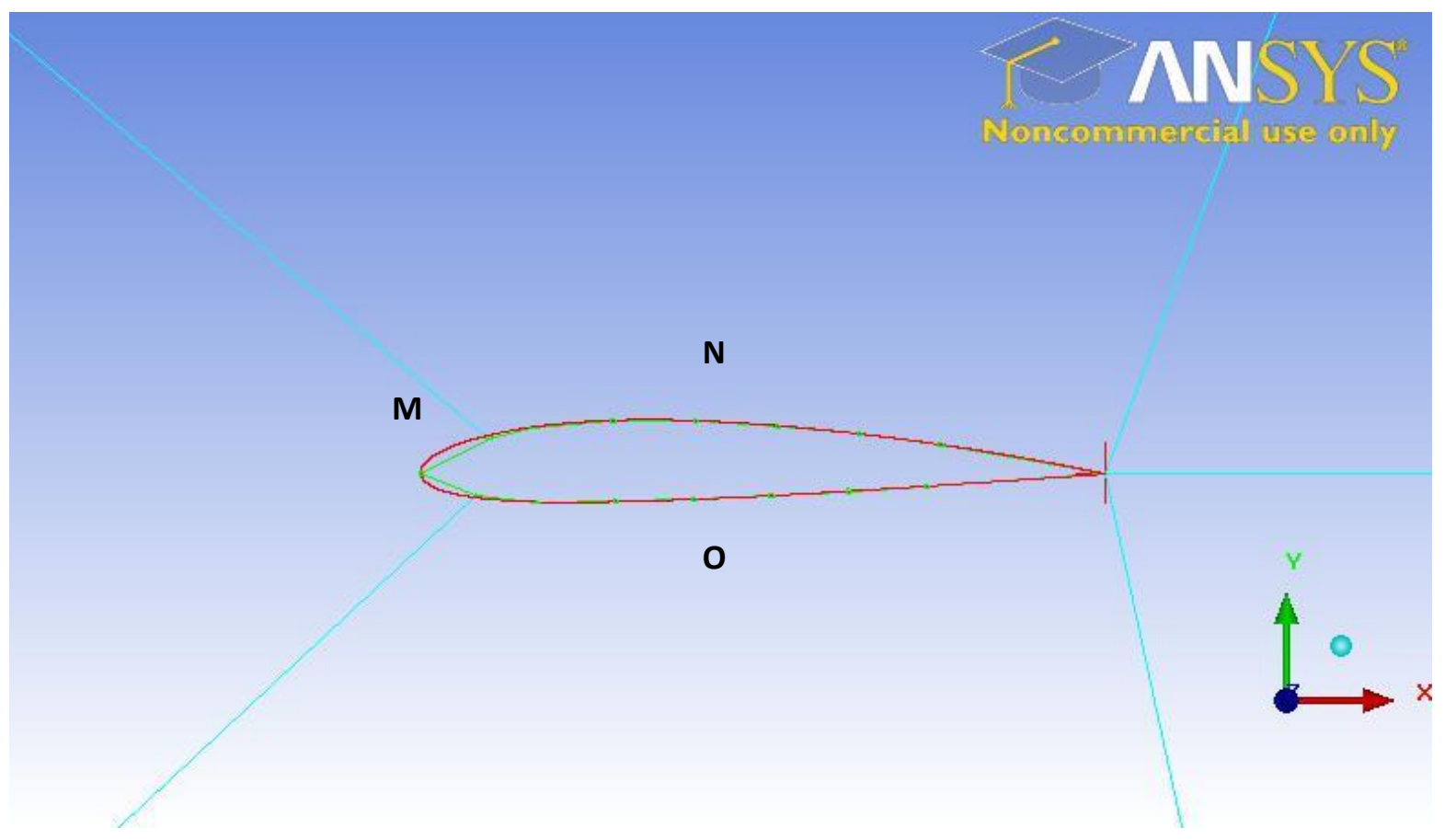

Figure 72: NACA 2412 Geometry 
Table 8: NACA 2412 Mesh Information

\begin{tabular}{|c|c|c|c|c|c|c|c|}
\hline Edge & Length & Nodes & Mesh Law & Spacing 1 & Ratio 1 & Spacing 2 & Ratio 2 \\
\hline \multicolumn{8}{|c|}{ DOMAIN } \\
\hline $\mathbf{A}$ & 29.1372 & 120 & Uniform & 0.184624 & 1.05 & 0.05 & 1.05 \\
\hline B & 25.7306 & 110 & Uniform & 0.05 & 1.05 & 0.05 & 1.05 \\
\hline C & 21.576 & 120 & Hyperbolic & 0.2 & 1.05 & 2 & 1.05 \\
\hline D & 20 & 180 & Hyperbolic & 2 & 1.1 & 0.05 & 1.05 \\
\hline $\mathbf{E}$ & 20 & 180 & Hyperbolic & 2 & 1.1 & 0.05 & 1.05 \\
\hline $\mathbf{F}$ & 24.6533 & 120 & Hyperbolic & 0.2 & 1.05 & 2 & 1.05 \\
\hline $\mathbf{G}$ & 21.7283 & 110 & Uniform & 0.05 & 1.05 & 0.05 & 1.05 \\
\hline \multicolumn{8}{|l|}{ FLUID } \\
\hline \multicolumn{8}{|c|}{ REGION } \\
\hline $\mathbf{H}$ & 20.4644 & 180 & Hyperbolic & 1.5 & 1.1 & 1E-06 & 0.05 \\
\hline I & 19.8352 & 180 & Hyperbolic & 1.5 & 1.1 & $1 \mathrm{E}-06$ & 0.05 \\
\hline $\mathbf{J}$ & 20.0428 & 180 & Hyperbolic & 1.5 & 1.1 & $1 \mathrm{E}-06$ & 0.05 \\
\hline K & 21.3309 & 180 & Hyperbolic & 1.5 & 1.1 & $1 \mathrm{E}-06$ & 0.05 \\
\hline $\mathbf{L}$ & 28.9911 & 120 & Hyperbolic & 0.007 & 1.05 & 2 & 1.1 \\
\hline
\end{tabular}


Table 9: NACA 2412 Nodal Distribution along the curves

\begin{tabular}{ccclcccc}
\hline Edge & Length & Nodes & Mesh Law & Spacing 1 & Ratio 1 & Spacing 2 & Ratio 2 \\
\cline { 1 - 2 } & & & & & & \\
M & 0.200126 & 120 & Hyperbolic & 0.005 & 1.05 & 0.005 \\
$\mathbf{N}$ & 0.91306 & 110 & Hyperbolic & 0.005 & 1.05 & 0.007 \\
$\mathbf{O}$ & 0.929445 & 110 & Hyperbolic & 0.005 & 1.05 & 0.007 \\
\hline
\end{tabular}




\section{Baseline Mesh Overlay}

Applying the mesh nodal distribution described in Table 8 and Table 9, the following are two dimensional views for the baseline mesh constructed.

View of Far-field Distribution

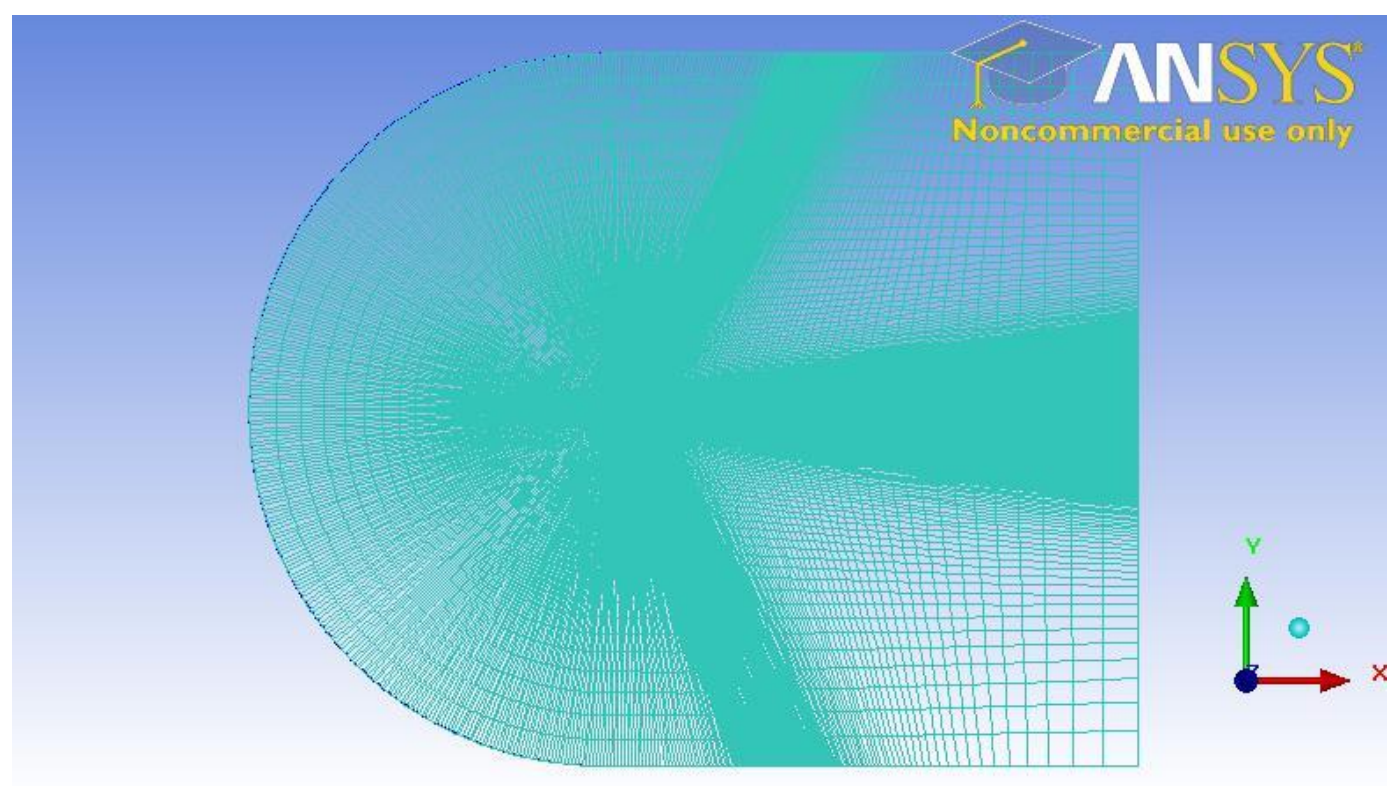

Figure 73: Baseline, Ideal Mesh, Farfield Distribution 


\section{Baseline NACA 2412 Geometry View}

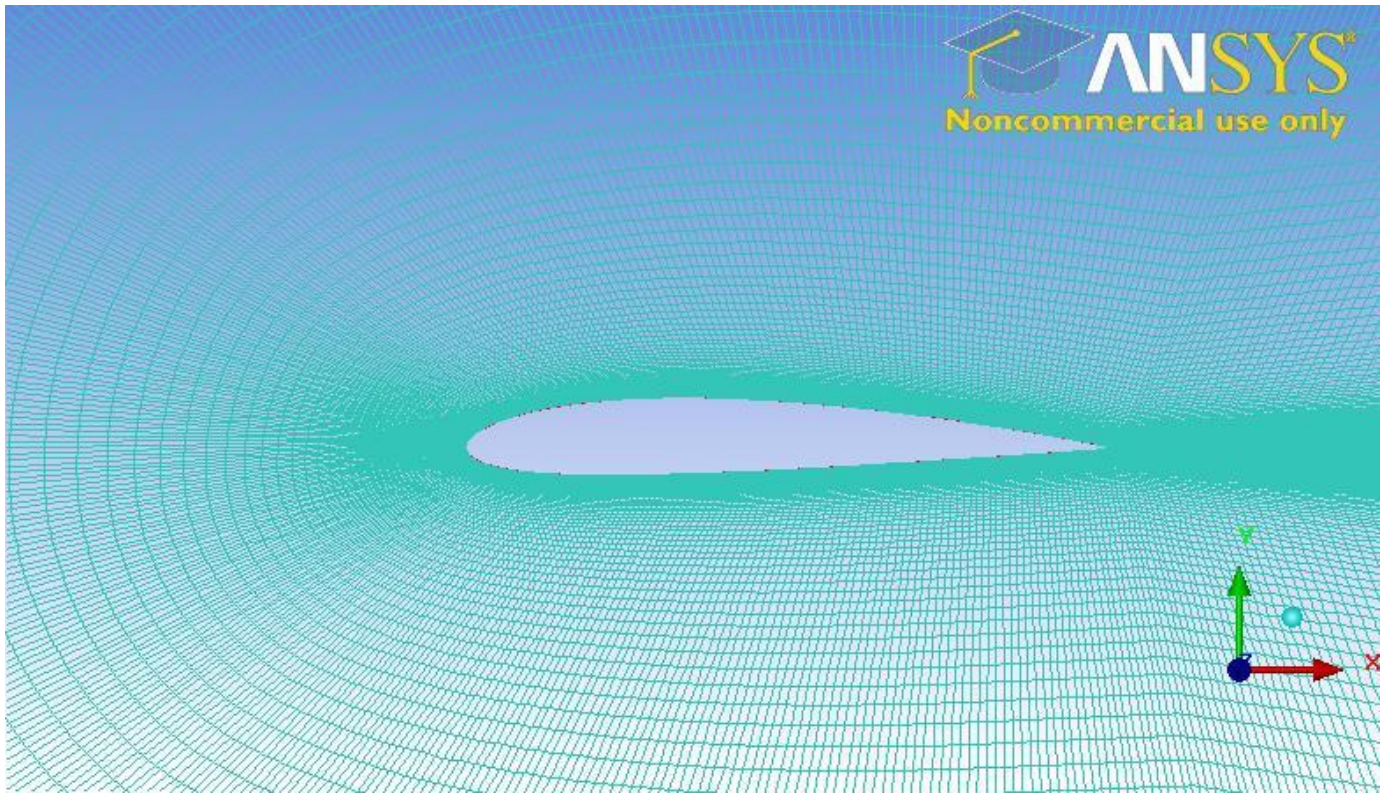

Figure 74: Baseline, Ideal Mesh, NACA 2412 Near wall Distribution

\section{Baseline Leading-Edge View}

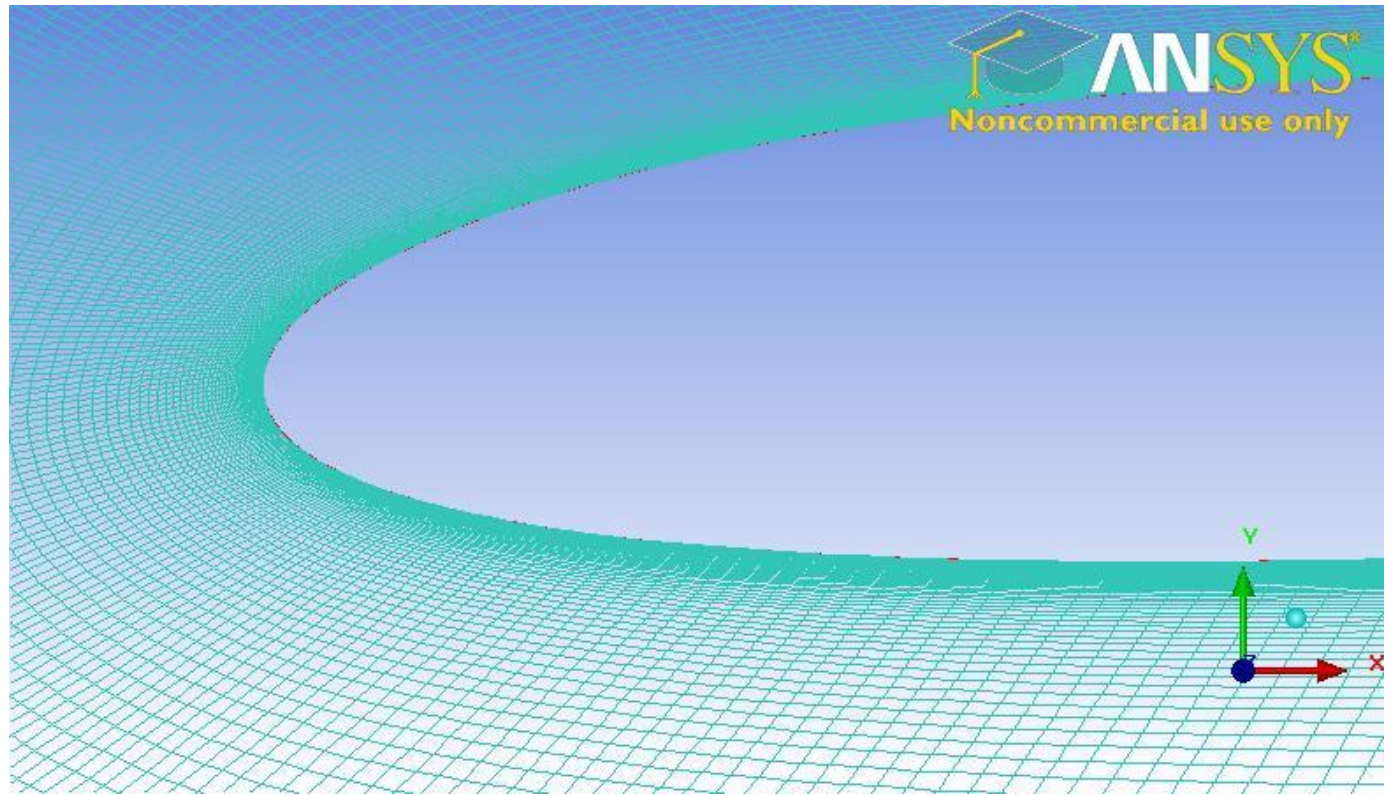

Figure 75: NACA2412 Leading Edge View 


\section{Baseline Trailing Edge View}

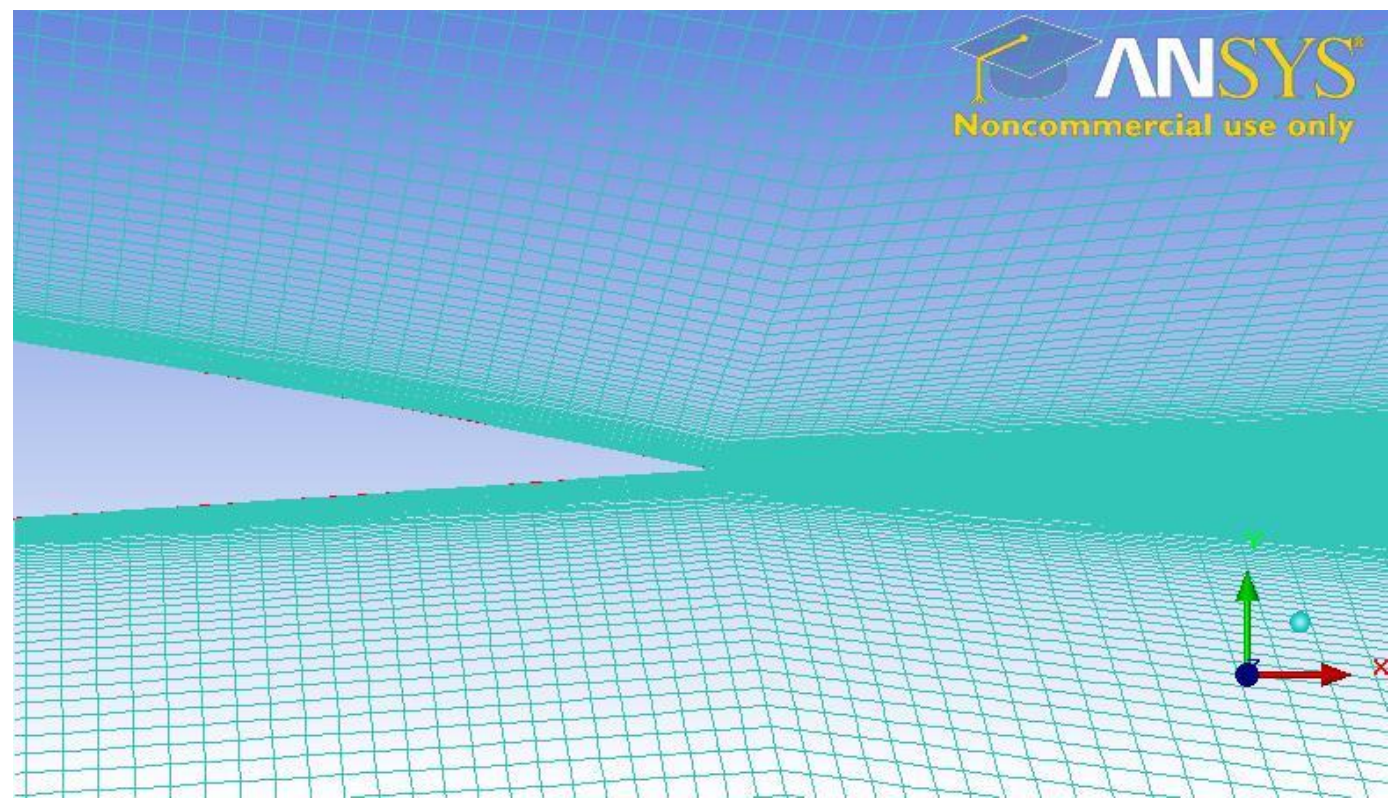

Figure 76: NACA2412 Trailing Edge View

Using the baseline mesh nodal distribution as a template, the spoiler-airfoil mesh was constructed to maintain consistency for computational purposes. The following sections provide the airfoil-spoiler geometry and mesh generation details. 


\section{Appendix B: Spoiler Mesh Details}

The following provides an overview of the blocking pattern utilized in the construction of the spoiler-airfoil mesh, along with nodal information provided in the next sections.

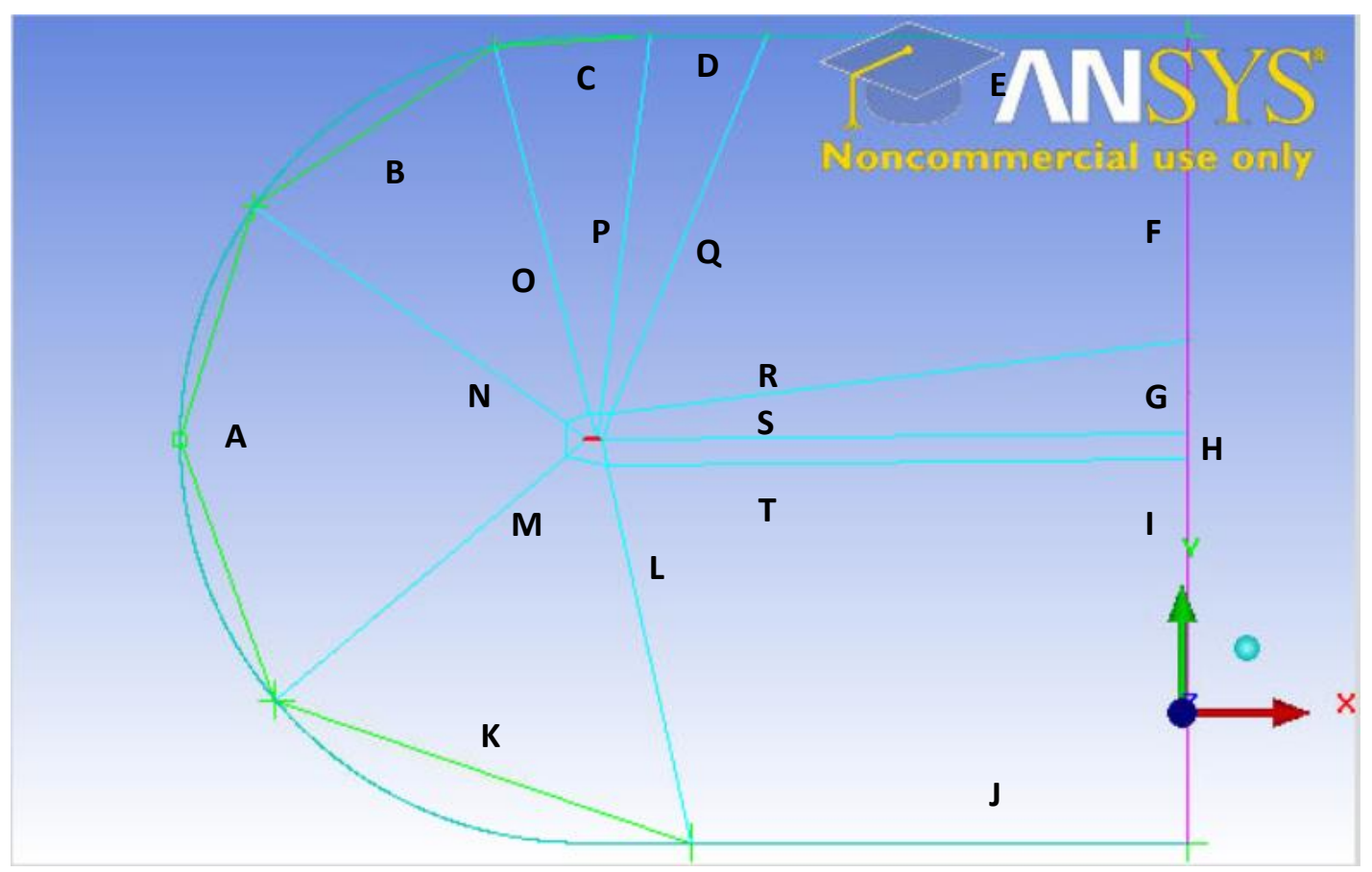

Figure 77: NACA2412 Airfoil-Spoiler Farfield Mesh Details 


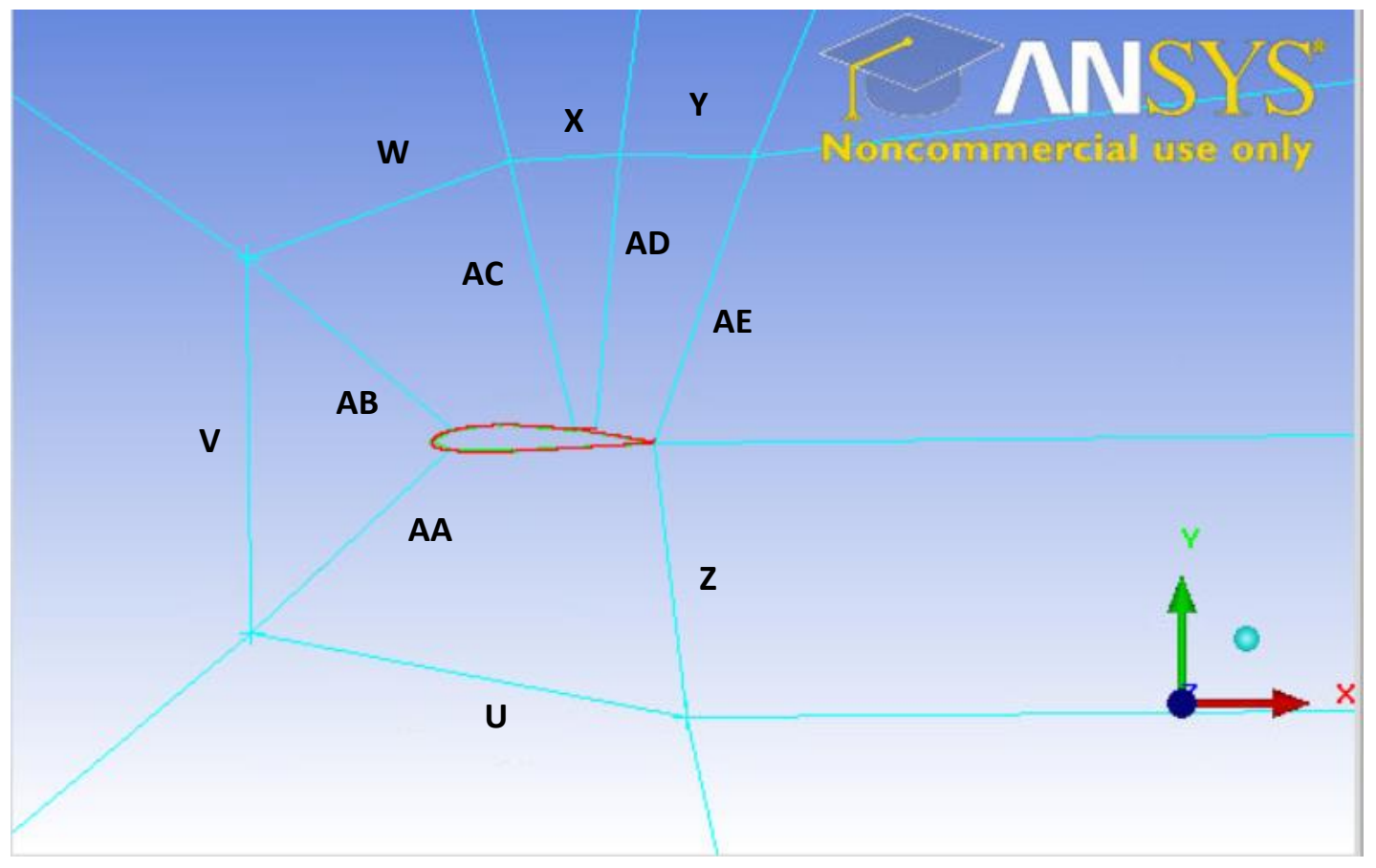

Figure 78: NACA2412 Airfoil-Spoiler Boundary Layer Mesh Details

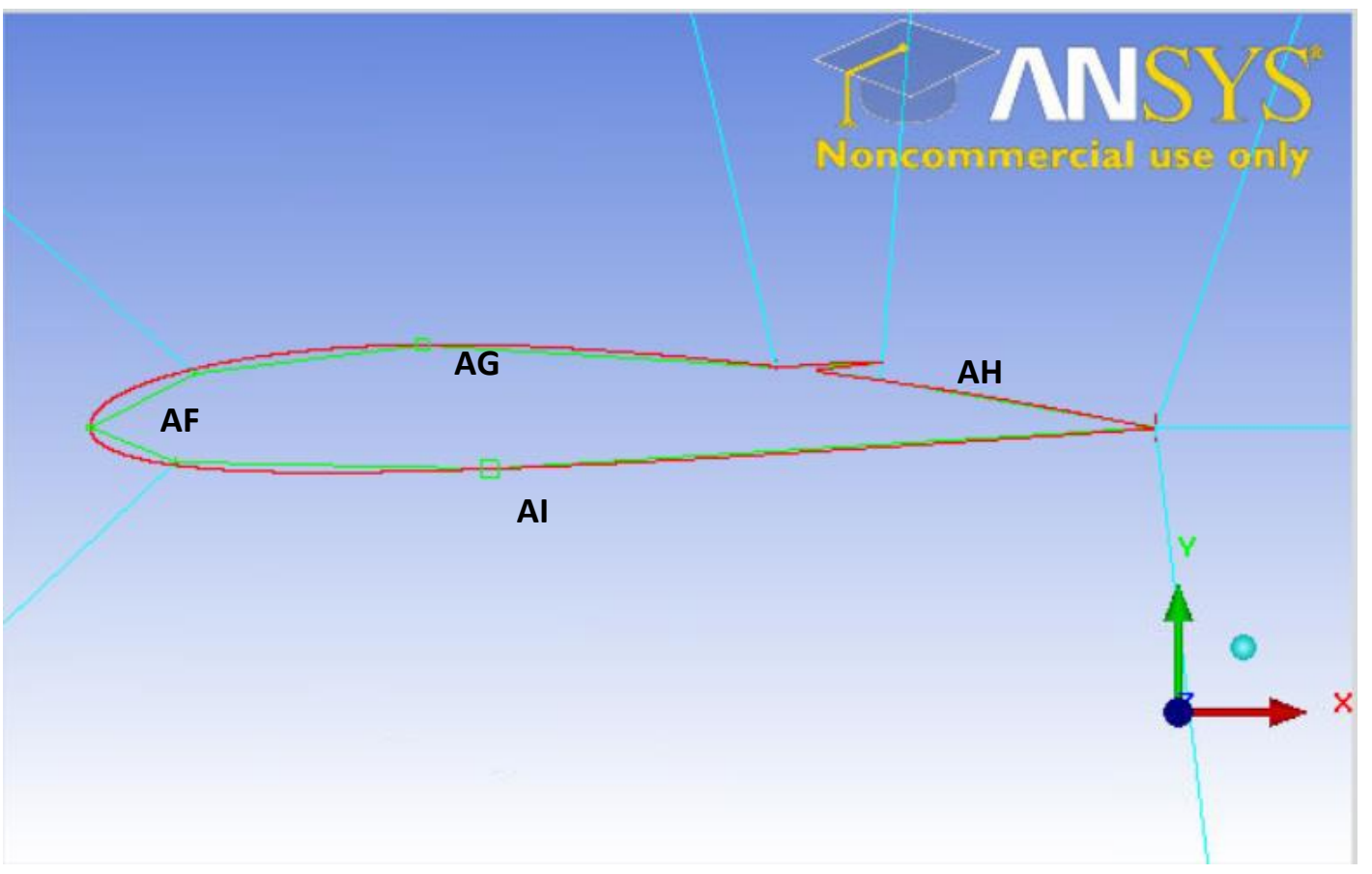

Figure 79: NACA2412 Airfoil-Spoiler Mesh Details 


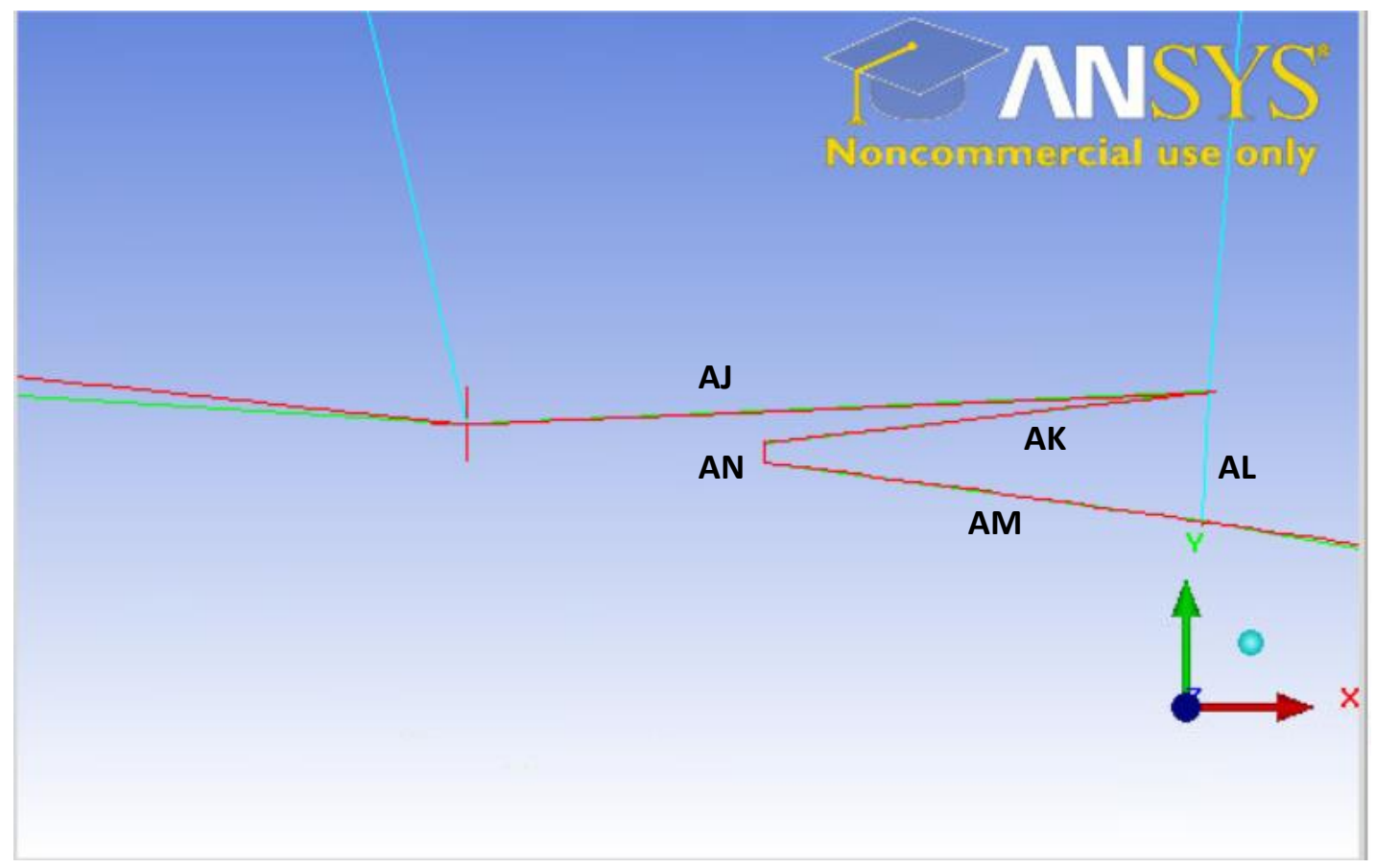

Figure 80: Spoiler Mesh Details 
Table 11: NACA 2412 Baseline Mesh Details

\begin{tabular}{|c|c|c|c|c|c|c|c|}
\hline Edge & Length & Nodes & Mesh Law & Spacing 1 & Ratio 1 & Spacing 2 & Ratio 2 \\
\hline $\bar{A}$ & 26.3484 & 120 & Uniform & 0.184624 & 1.05 & 0.05 & 1.05 \\
\hline B & 14.7312 & 80 & Uniform & 0.05 & 1.05 & 0.05 & 1.05 \\
\hline $\mathrm{C}$ & 7.76297 & 40 & Uniform & 0.05 & 1.05 & 0.05 & 1.05 \\
\hline D & 5.77739 & 50 & Uniform & 0.05 & 1.05 & 0.05 & 1.05 \\
\hline $\mathbf{E}$ & 20.8532 & 120 & Hyperbola & 0.184624 & 1.05 & 2 & 1.05 \\
\hline $\mathbf{F}$ & 15.0862 & 40 & Hyperbola & 2 & 1.1 & 0.016824 & 1.05 \\
\hline G & 2.56989 & 140 & Hyperbola & 0.01624 & 1.05 & 0.0100245 & 1.05 \\
\hline $\mathbf{H}$ & 1.25152 & 140 & Uniform & 0.01624 & 2 & 0.01 & 1.05 \\
\hline I & 19.0215 & 40 & Hyperbola & 3 & 1.1 & 0.0140625 & 1.1 \\
\hline $\mathbf{J}$ & 24.6533 & 120 & Hyperbola & 0.184624 & 1.05 & 2 & 1.05 \\
\hline $\mathbf{K}$ & 22.6939 & 130 & Uniform & 0.05 & 1.05 & 0.05 & 1.05 \\
\hline $\mathbf{L}$ & 19.2204 & 40 & Uniform & 1.5 & 1.1 & 0.1 & 1.05 \\
\hline M & 18.8174 & 40 & Hyperbola & 1.5 & 1.1 & 0.1 & 1.05 \\
\hline $\mathbf{N}$ & 18.839 & 40 & Hyperbola & 1.5 & 1.1 & 0.1 & 1.05 \\
\hline $\mathbf{O}$ & 18.8421 & 40 & Hyperbola & 1.5 & 1.1 & 0.1 & 1.05 \\
\hline $\mathbf{P}$ & 18.8607 & 40 & Hyperbola & 1.5 & 1.1 & 0.1 & 1.05 \\
\hline $\mathbf{Q}$ & 20.2248 & 40 & Hyperbola & 1.5 & 1.1 & 0.1 & 1.05 \\
\hline $\mathbf{R}$ & 28.7667 & 120 & Hyperbola & 0.01 & 1.05 & 0.8 & 1.1 \\
\hline $\mathbf{S}$ & 28.9924 & 120 & Hyperbola & 0.00788 & 1.05 & 0.8 & 1.1 \\
\hline $\mathbf{T}$ & 28.8449 & 120 & Hyperbola & 0.007 & 1.05 & 0.8 & 1.1 \\
\hline
\end{tabular}




\begin{tabular}{|c|c|c|c|c|c|c|c|}
\hline $\mathbf{U}$ & 2.00872 & 130 & Uniform & 0.005 & 1.05 & 0.005 & 1.05 \\
\hline V & 1.7022 & 120 & Uniform & 0 & 2 & 0 & 2 \\
\hline W & 1.2632 & 80 & Uniform & 0.005 & 1.05 & 0.005 & 1.05 \\
\hline $\mathbf{X}$ & 0.496695 & 40 & Uniform & 0.005 & 1.05 & 0.005 & 1.05 \\
\hline $\mathbf{Y}$ & 0.610711 & 50 & Uniform & 0.005 & 1.05 & 0.005 & 1.05 \\
\hline $\mathbf{Z}$ & 1.25007 & 140 & Hyperbola & 0.1 & 1.05 & 0.005 & 1.05 \\
\hline $\mathbf{A A}$ & 1.22413 & 140 & Hyperbola & 0.1 & 1.05 & $1 \mathrm{e}-06$ & 1.05 \\
\hline $\mathbf{A B}$ & 1.21833 & 140 & Hyperbola & 0.1 & 1.05 & $1 \mathrm{e}-06$ & 1.05 \\
\hline $\mathbf{A C}$ & 1.25339 & 140 & Hyperbola & 0.1 & 1.05 & $1 \mathrm{e}-06$ & 1.05 \\
\hline AD & 1.24975 & 140 & Hyperbola & 0.1 & 1.05 & $1 \mathrm{e}-06$ & 1.05 \\
\hline $\mathbf{A E}$ & 1.36974 & 259 & Hyperbola & $1 e-06$ & 1.05 & 0.15 & 1.05 \\
\hline
\end{tabular}


Table 12: NACA2412 Airfoil-Spoiler Mesh Details Cont'd

\begin{tabular}{|c|c|c|c|c|c|c|c|}
\hline Edge & Length & Nodes & Mesh Law & Spacing 1 & Ratio 1 & Spacing 2 & Ratio 2 \\
\hline $\mathbf{A F}$ & 0.20012 & 120 & Hyperbola & 0.005 & 1.05 & 0.005 & 1.05 \\
\hline $\mathbf{A G}$ & 0.55255 & 80 & Hyperbola & 0.005 & 1.05 & 0.005 & 1.05 \\
\hline $\mathbf{A H}$ & 0.2638 & 50 & Hyperbola & 0.003 & 1.05 & 0.007 & 1.05 \\
\hline AI & 0.929045 & 130 & Hyperbola & 0.005 & 1.05 & 0.007 & 1.05 \\
\hline AJ & 0.1 & 40 & Hyperbola & 0.005 & 1.05 & 0.003 & 1.05 \\
\hline AK & 0.0602234 & 40 & Hyperbola & 0.002 & 1.05 & 0.002 & 1.05 \\
\hline AL & 0.01712 & 120 & Hyperbola & $1 \mathrm{e}-06$ & 1.05 & $1 \mathrm{e}-06$ & 1.05 \\
\hline $\mathbf{A M}$ & 0.05959 & 40 & Hyperbola & 0.002 & 1.05 & 0.003 & 1.05 \\
\hline $\mathbf{A N}$ & 0.0026 & 120 & Hyperbola & $1 \mathrm{e}-06$ & 1.05 & $1 \mathrm{e}-06$ & 1.05 \\
\hline
\end{tabular}


NACA2412 Airfoil-Spoiler Mesh

Applying the mesh nodal distribution described in the previous section; the following are a compilation of views of the NACA2412-Spoiler constructed geometry with the mesh overlay.

\section{NACA2412-Spoiler Far-field Overview}

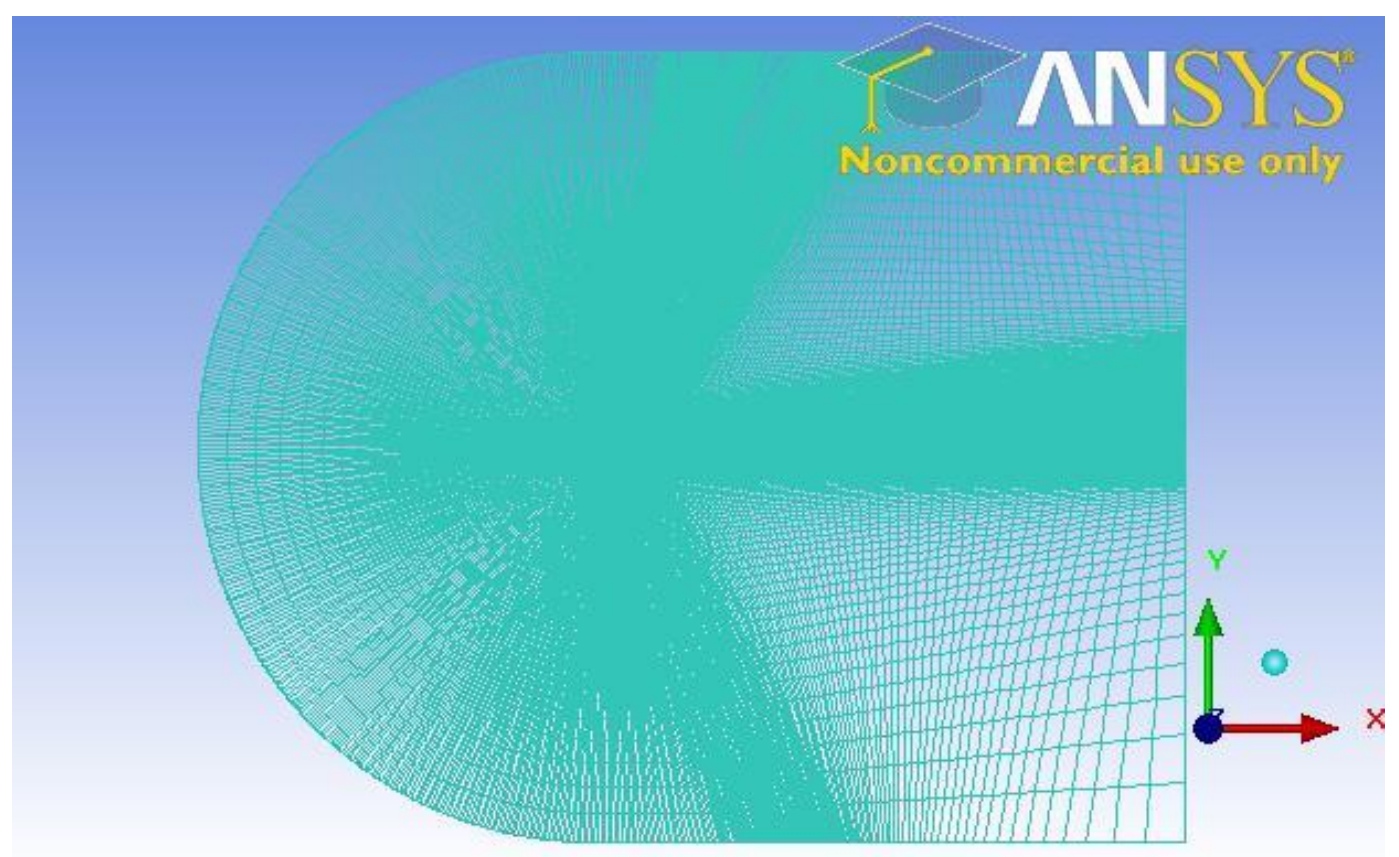

Figure 81: NACA2412 airfoil-Spoiler Farfield Overview 
NACA2412-Spoiler Leading Edge View

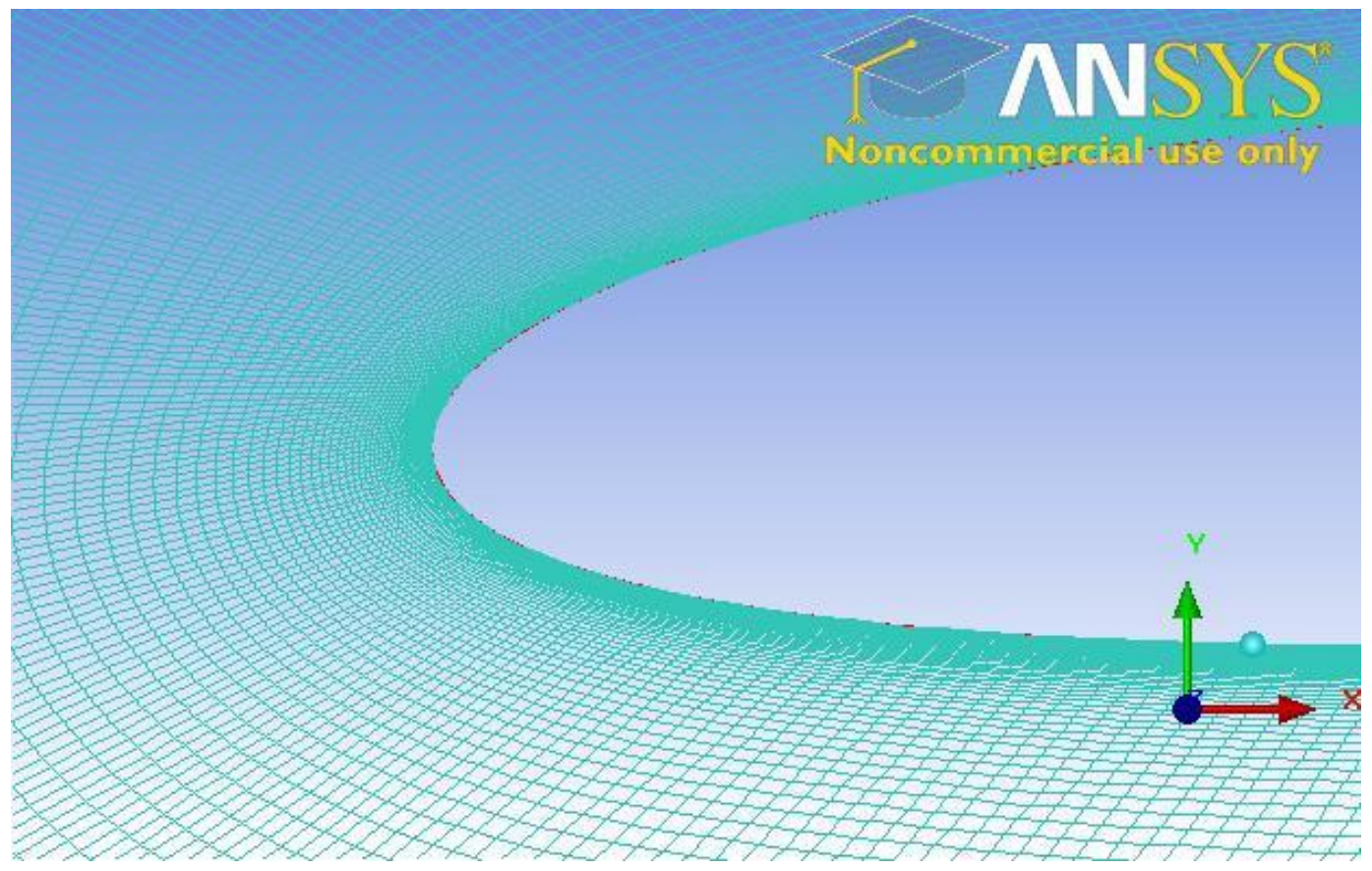

Figure 82: NACA2412-Spoiler Leading Edge View

\section{NACA2412-Spoiler Trailing Edge View 1}

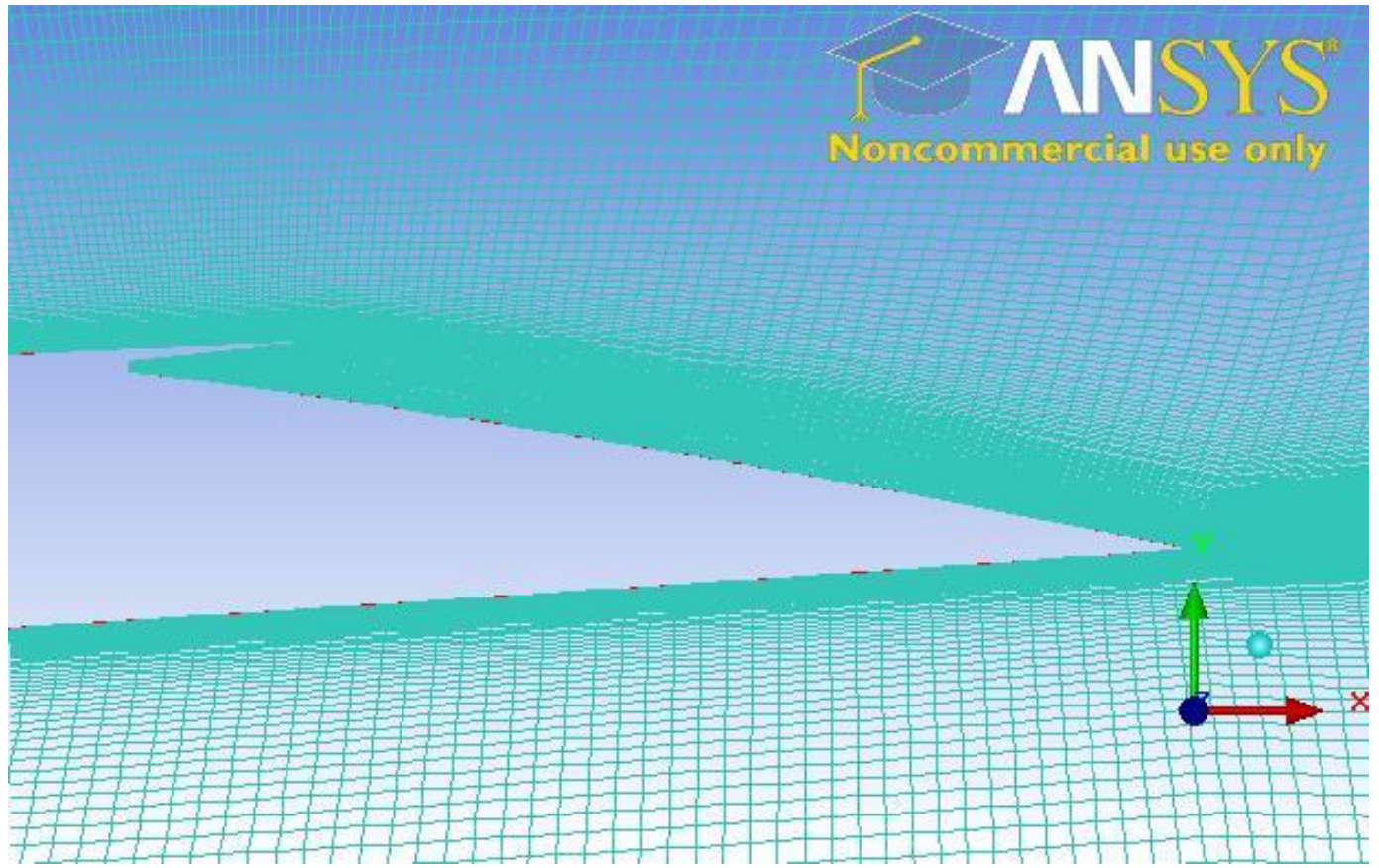

Figure 83: NACA2412-Spoiler Trailing Edge View 1 
NACA2412-Spoiler Trailing Edge View 2

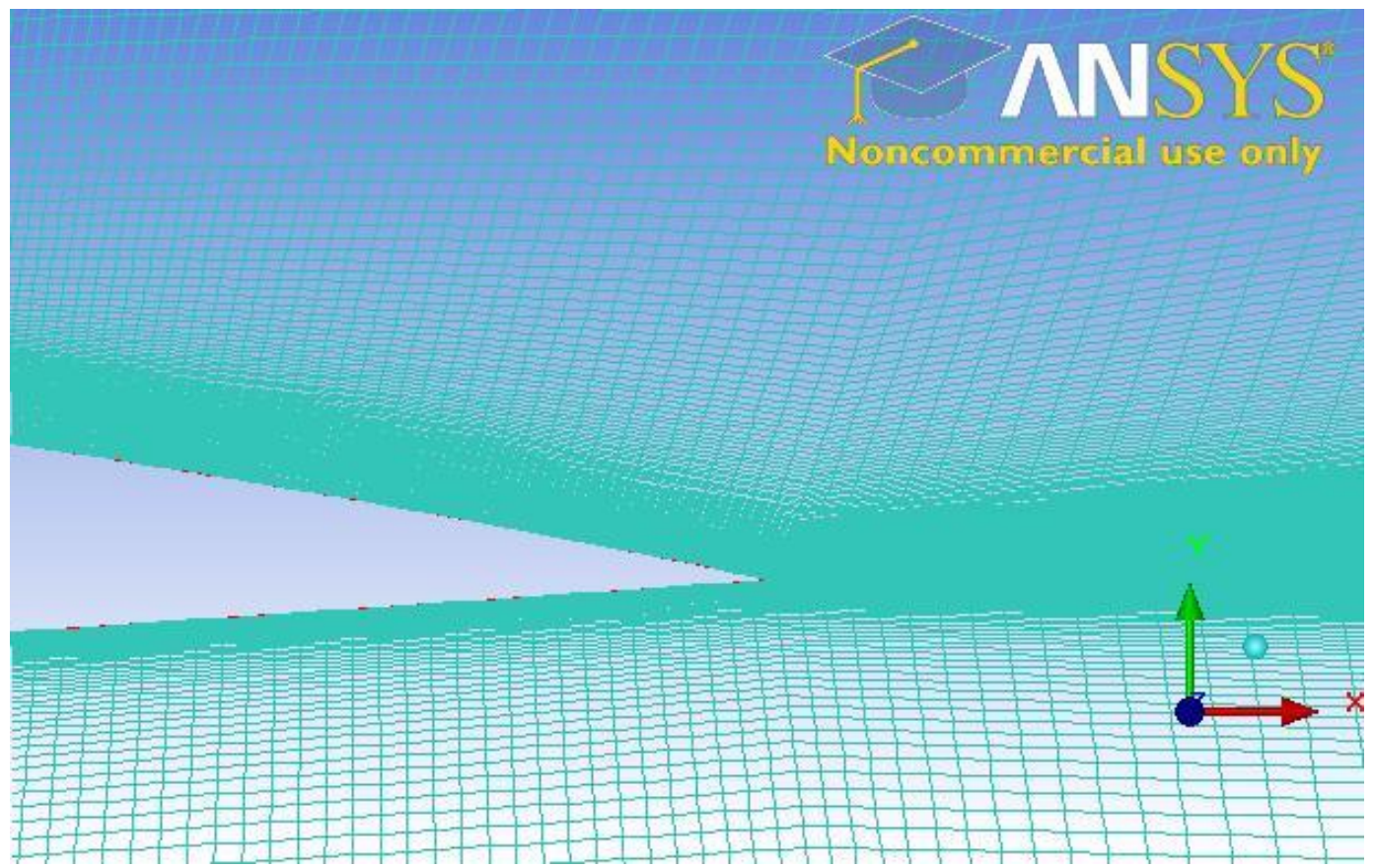

Figure 84: NAC2412-Spoiler Trailing Edge View 2

\section{Spoiler View}

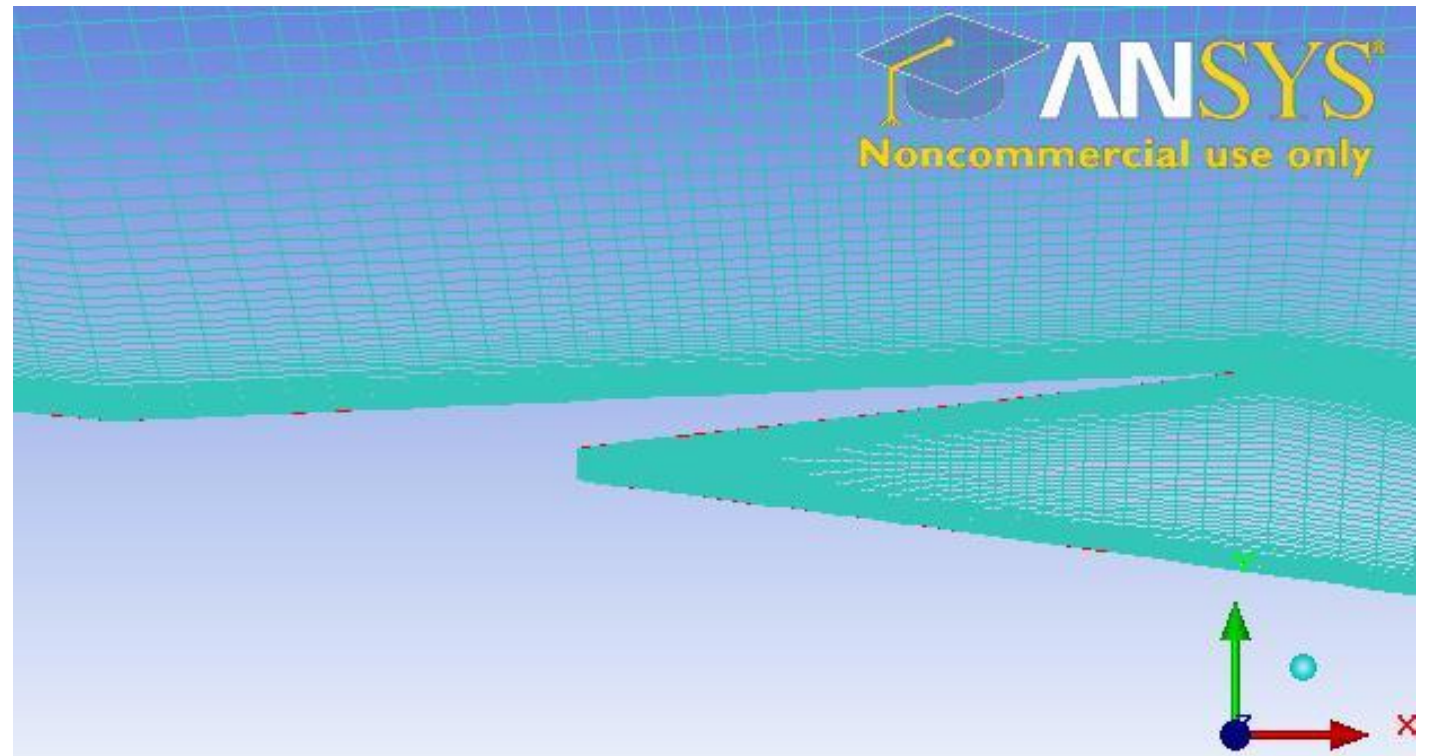

Figure 85: Spoiler View 


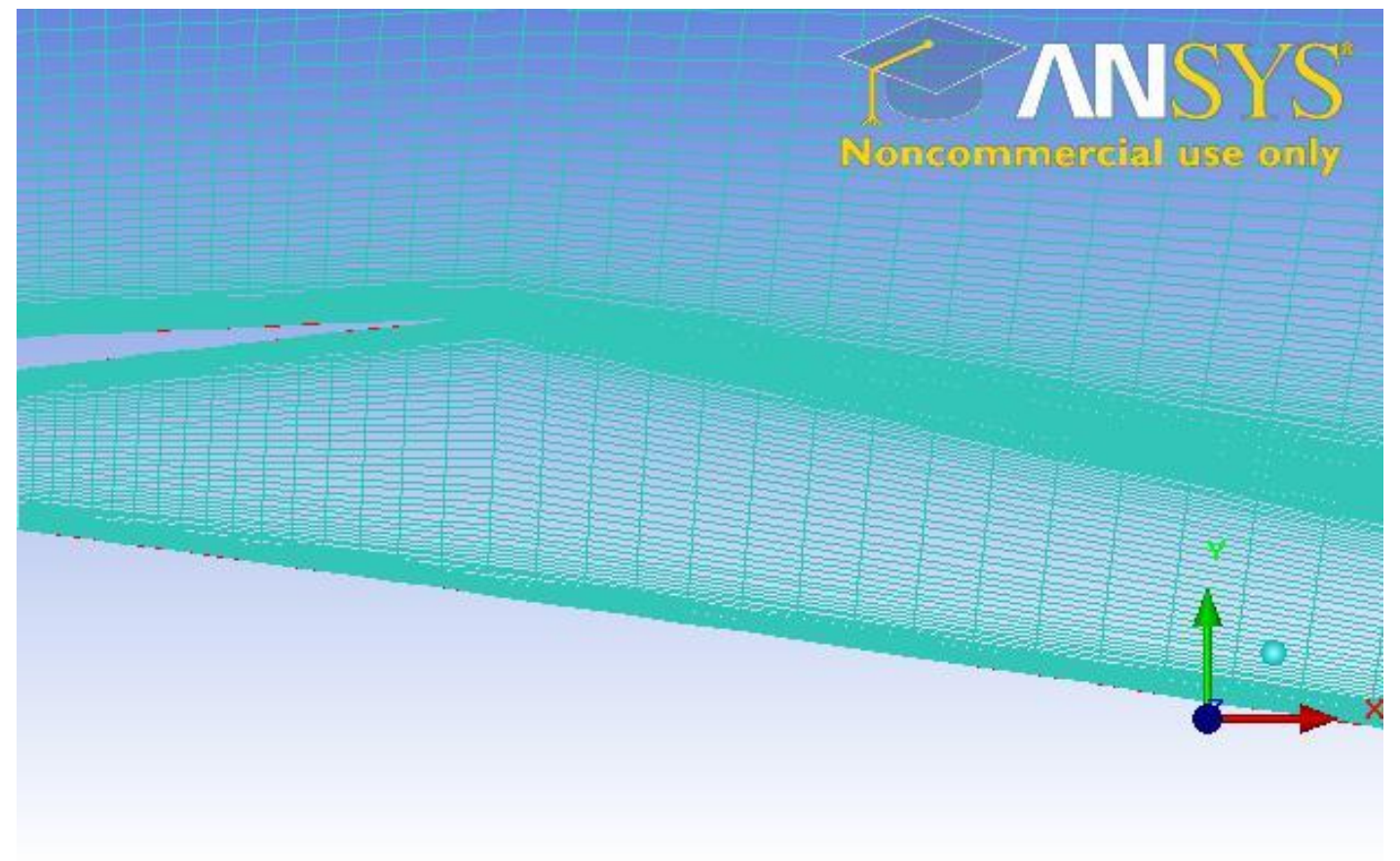

Figure 86: Mesh Details Near Spoiler Tip

\section{ANSYS ICEM CFD DEFAULT PARAMETERS:}

- To ensure a smooth leading edge and mesh overlay with the geometry, the NACA 2412 Coordinate points were imported into ANSYS ICEM CFD 14.0 with a tolerance of 1E14.

- To ensure the wall shear stress and pressure coefficient plots had shown smooth trends, the triangulation setting was set to 1E-06 (within ANSYS ICEM CFD 14.0 the path is the following: Setting > Model > Triangulation tolerance) 


\section{Appendix C Spoiler Performance Results:}

\section{$\underline{\text { Alpha }=16^{0:}}$}

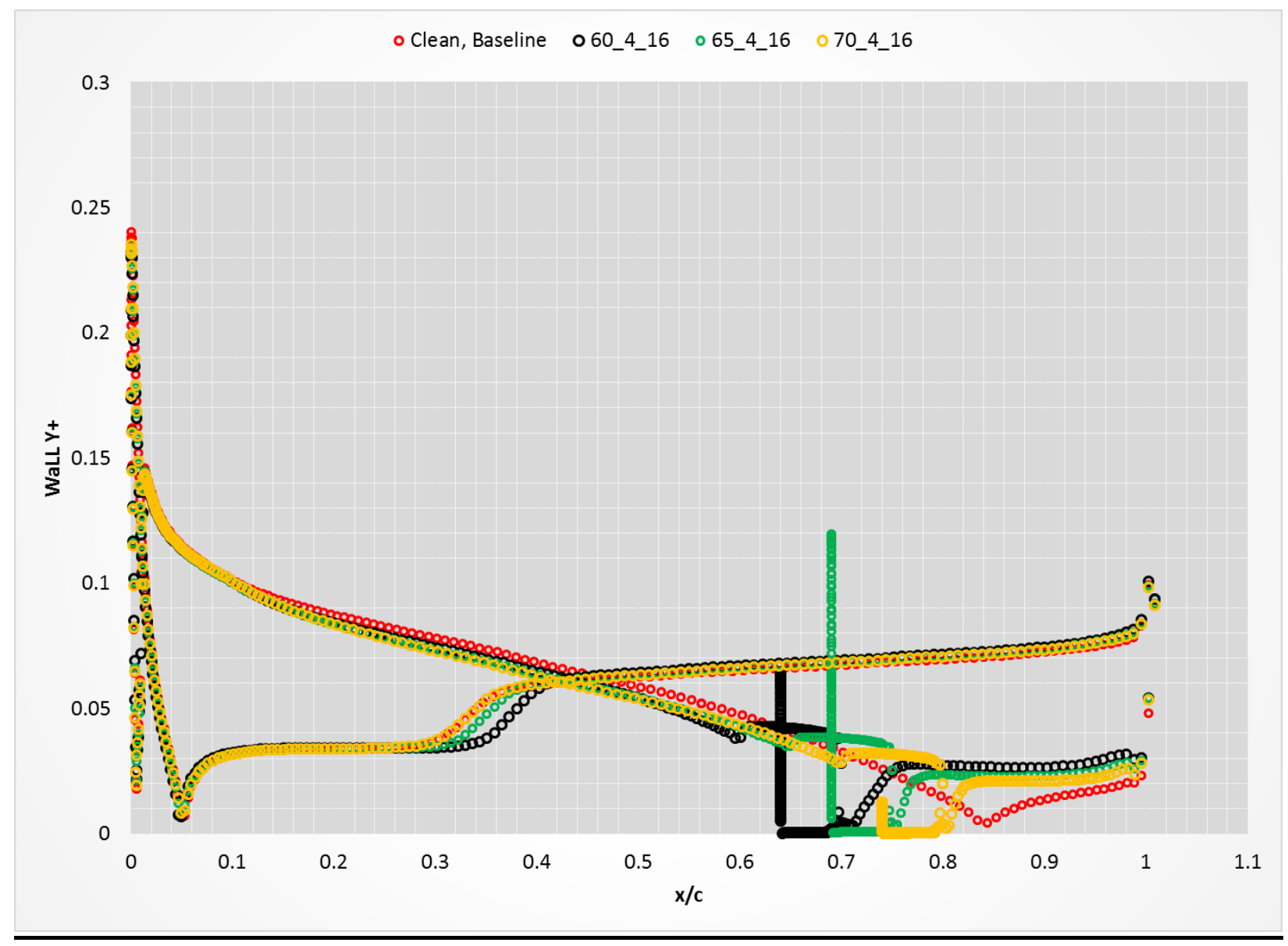

Figure 87: NACA 2412 Spoiler Study, Wall Y+, Spoiler Deflection = 4 degrees, Alpha = 16 Degrees, Transition SST Model, $R e=3.79 E 6$ 


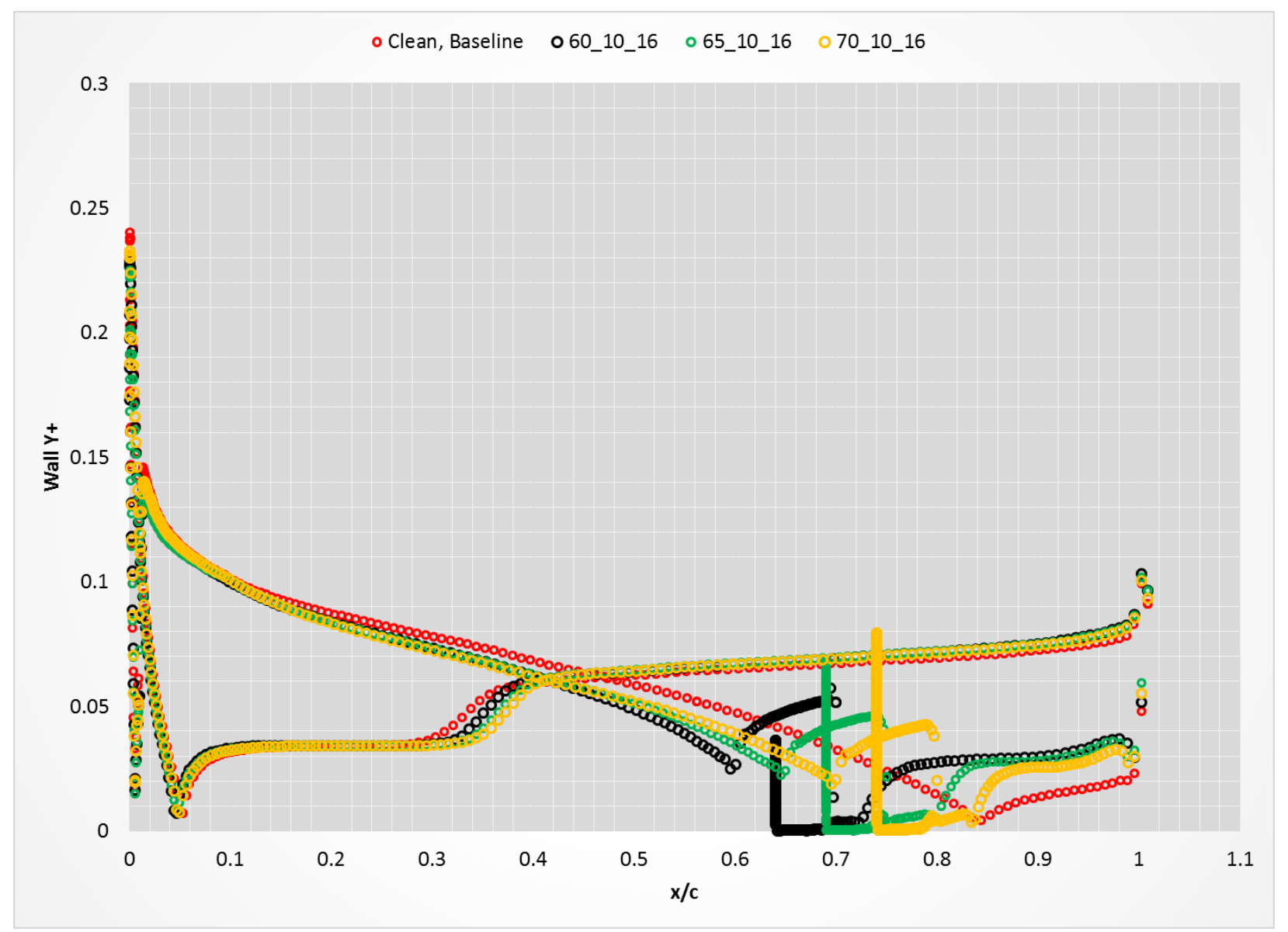

Figure 88: NACA 2412 Spoiler Study, Wall Y+, Spoiler Deflection = 10 degrees, Alpha = 16 Degrees, Transition SST Model, $R e=3.79 E 6$ 


\section{$\underline{\text { Alpha }}=17^{\circ}$, Spoiler Deflection $=10^{0:}$}

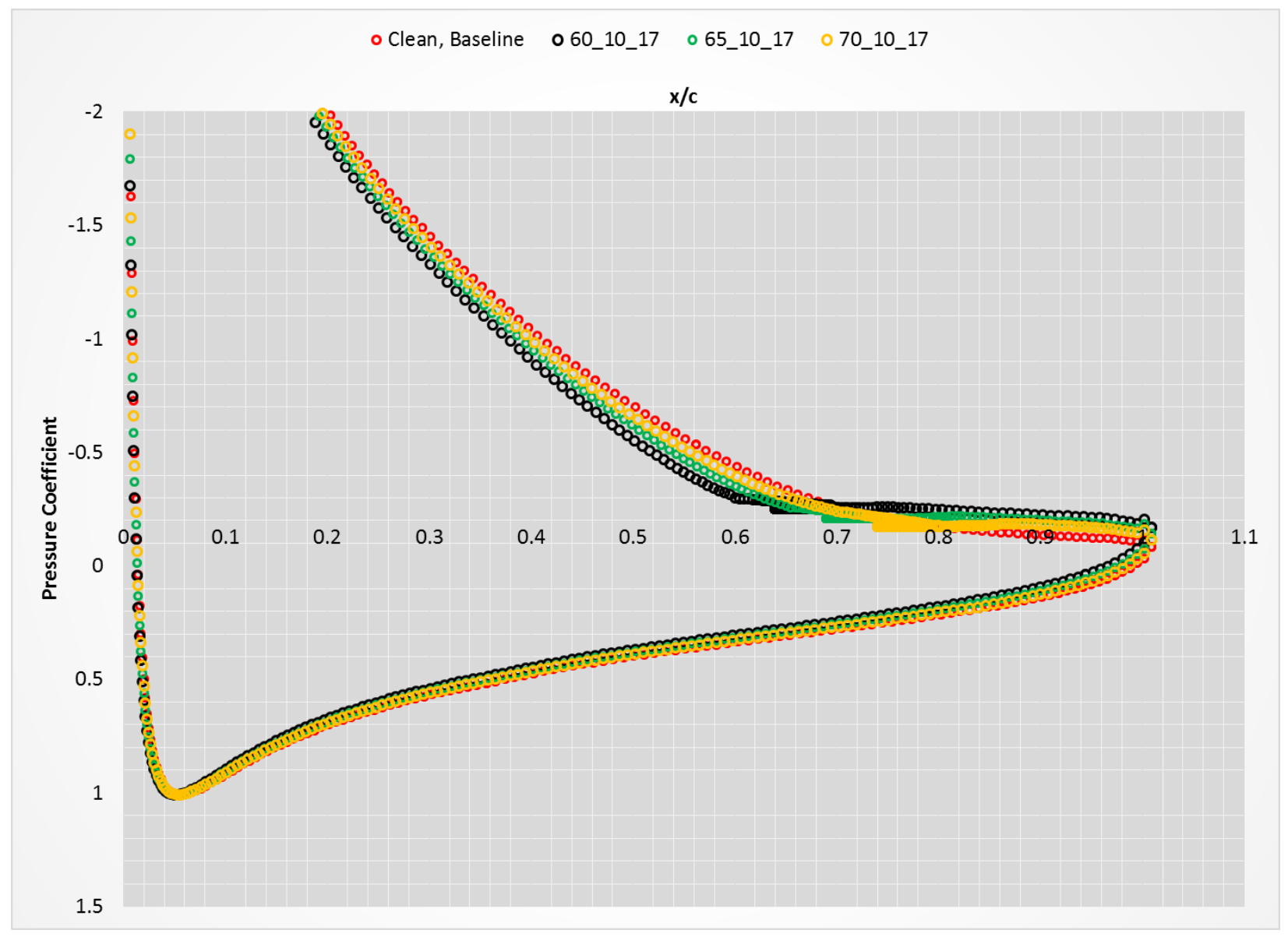

Figure 89:Pressure Coefficient, Alpha=17 degrees, Spoiler deflection=10 degrees, $M=0.17, \operatorname{Re}=3.79 E 6$, Transition SST 

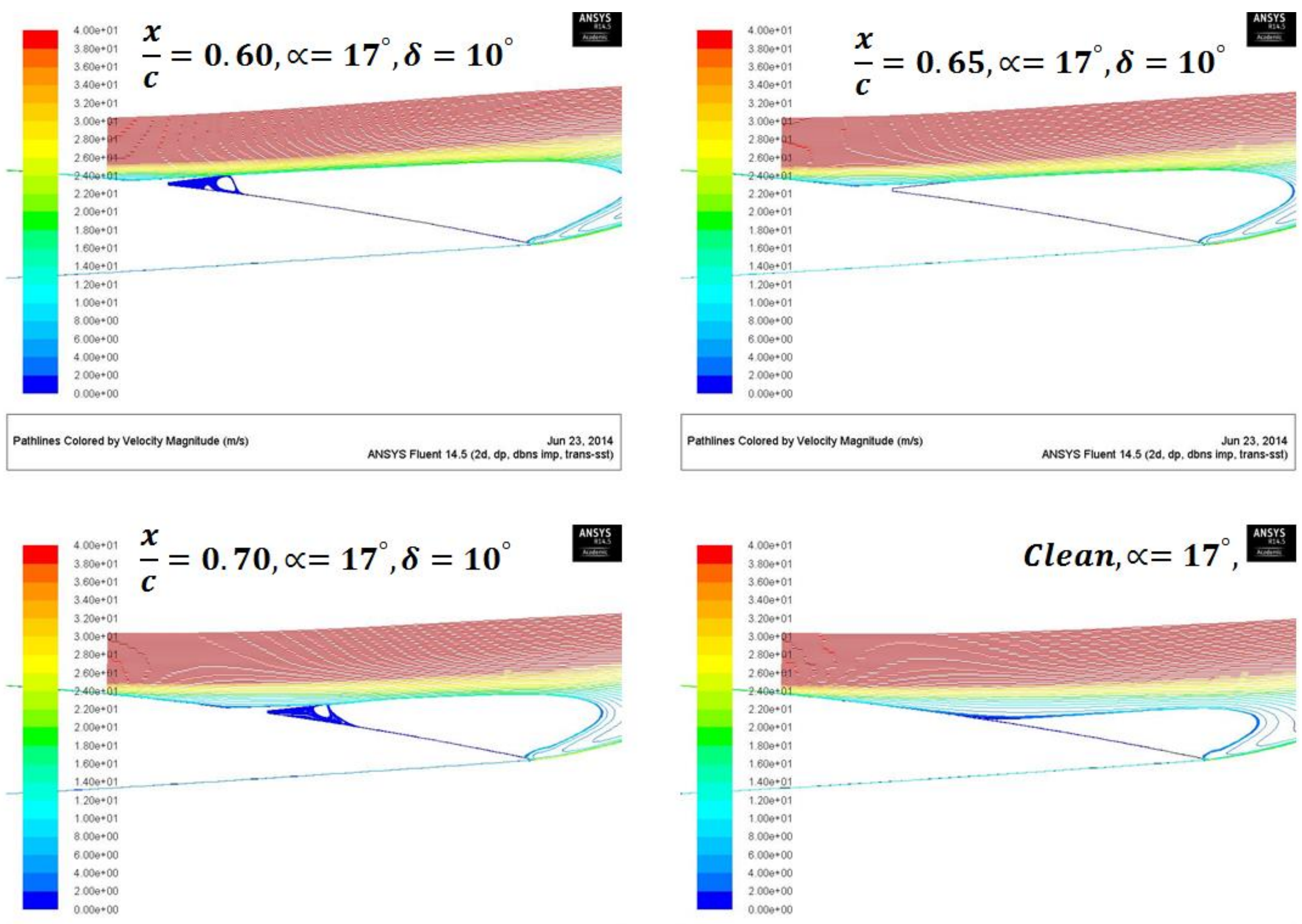


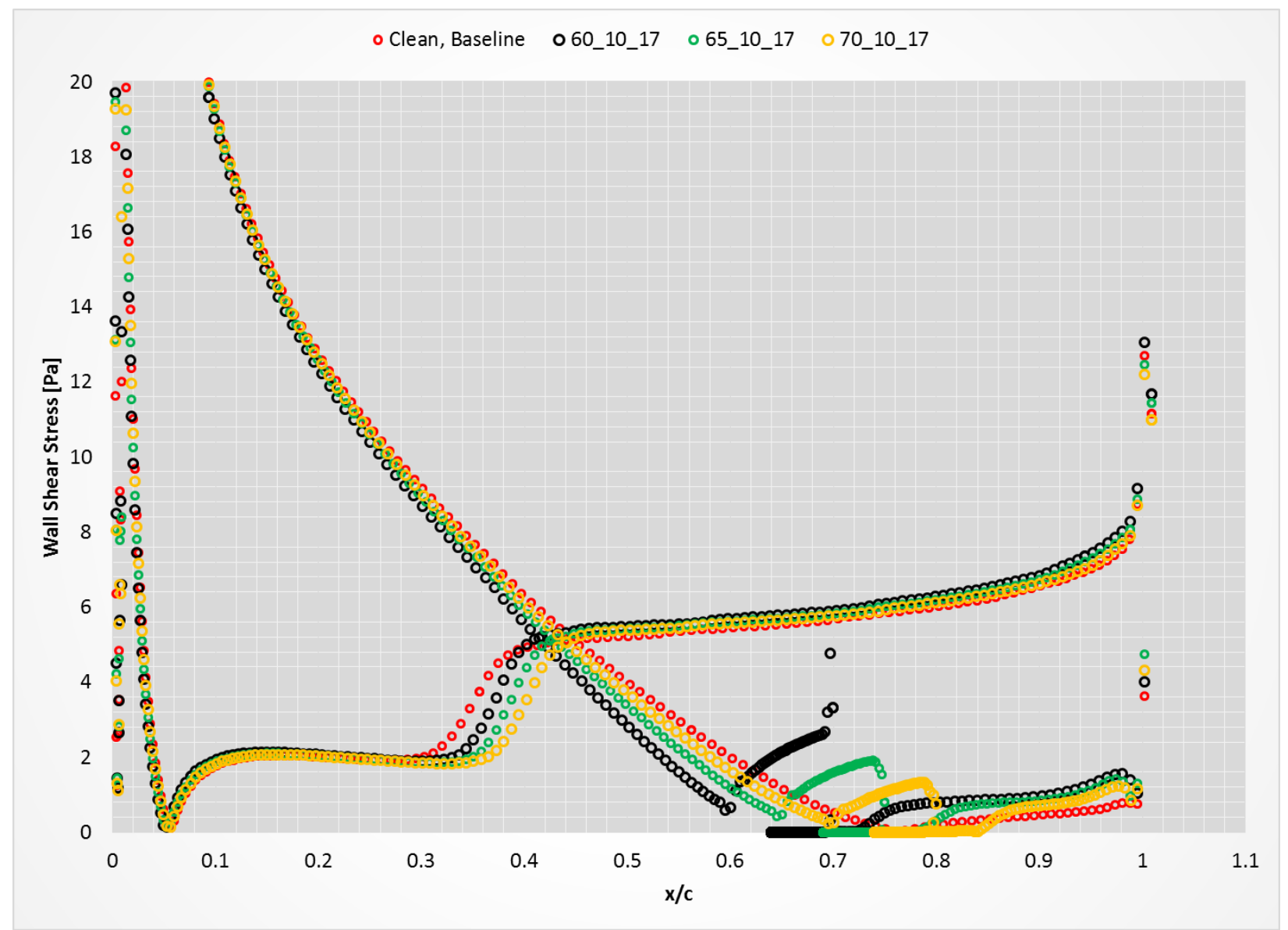

Figure 91: Wall shear stress plot, Alpha=17, Spoiler Deflection=10, $M=0.17, \operatorname{Re}=3.79 E 6$, Transition SST 


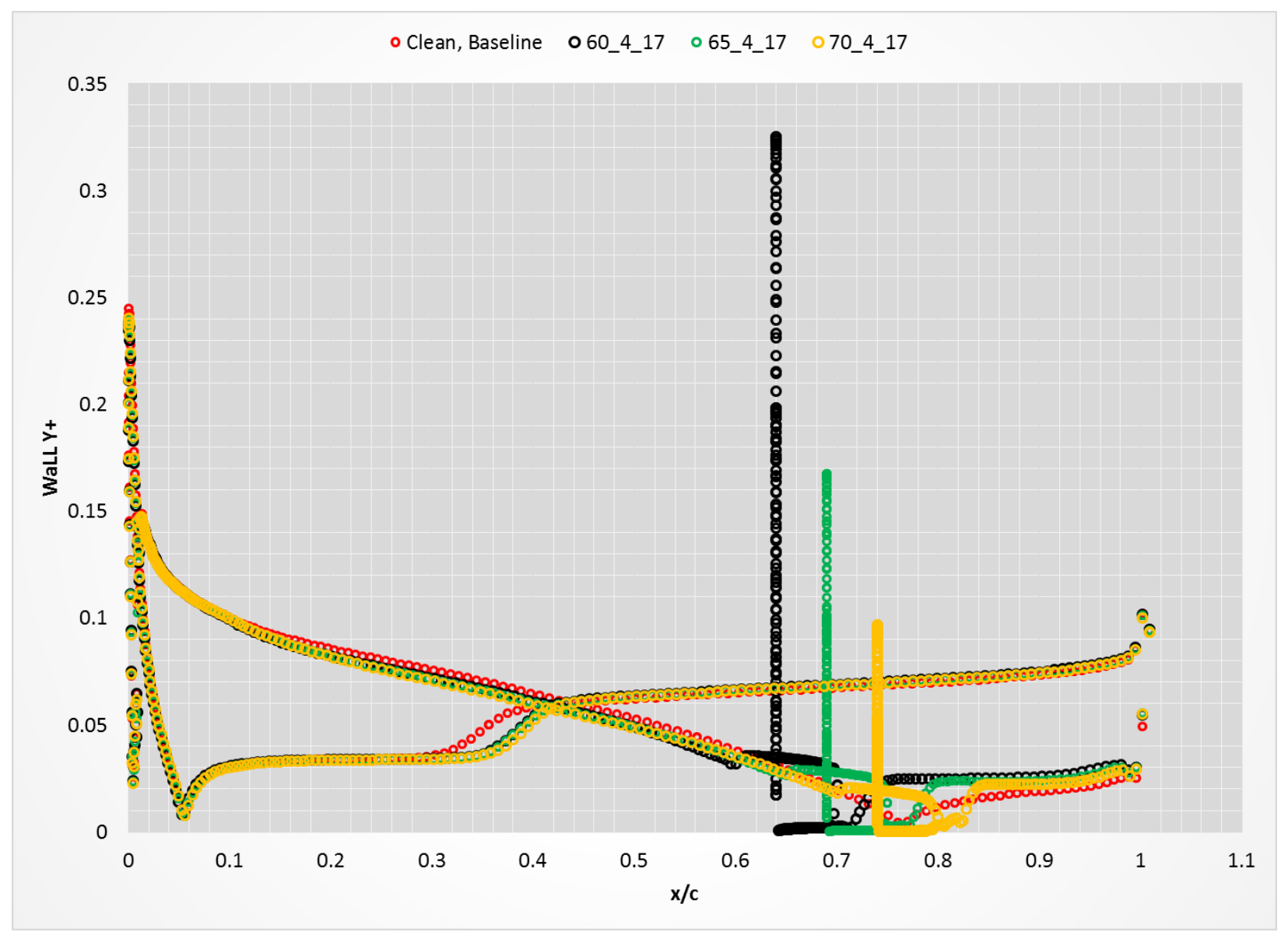

Figure 92: Wall Y+, Alpha=17, Spoiler Deflection=4, $M=0.17$, Re=3.79E6, Transition SST 


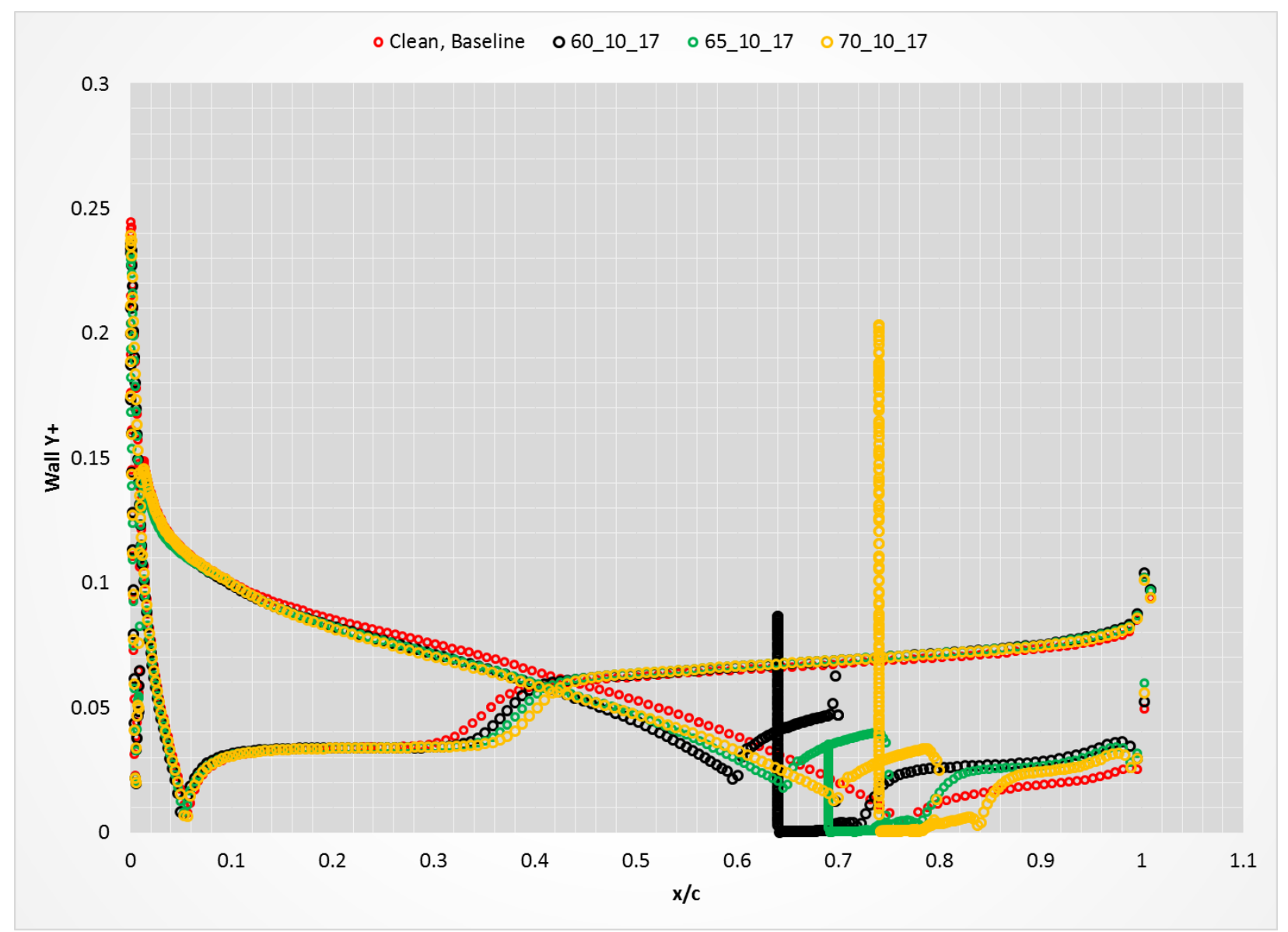

Figure 93: Wall Y+, Alpha=17, Spoiler Deflection=10, $M=0.17, R e=3.79 E 6$, Transition SST 
$\underline{\text { Alpha }=18^{\circ}, \text { Spoiler Deflection }=10^{0:}}$

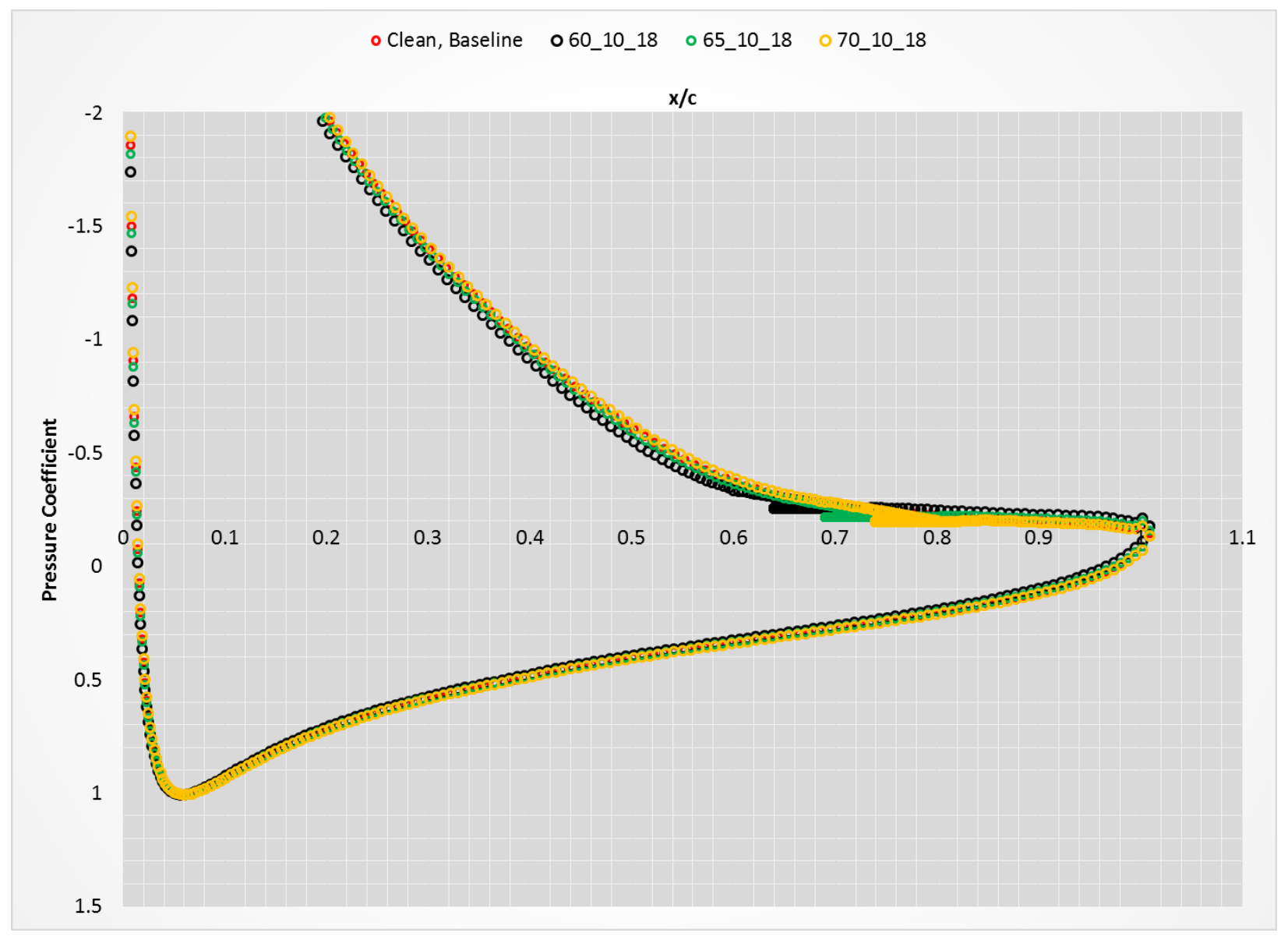

Figure 94: Pressure Coefficient, Alpha=18, Spoiler Deflection=10, M=0.17, Re=3.79E6, Transition SST 

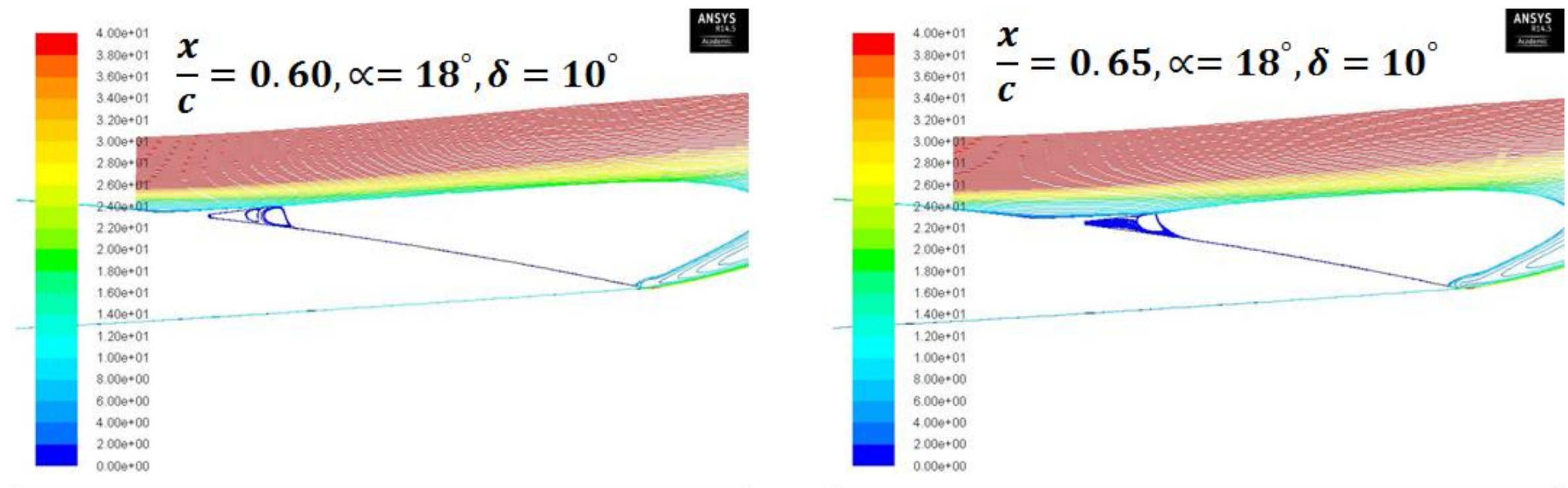

\begin{tabular}{|lr} 
Pathlines Colored by Velocity Magnilude (m/s) & Jun 23,2014 \\
& ANSYS Fluent 14.5 (2d. dp, dbns imp, trans-sst)
\end{tabular}

\begin{tabular}{|c|c|}
\hline Pathlines Colored by Velocily Magnitude $(\mathrm{m} / \mathrm{s})$ & $\begin{array}{r}\text { Jun } 23,2014 \\
\text { ANSYS Fluent } 14.5 \text { (2d, dp. dbns imp, trans-sst) }\end{array}$ \\
\hline
\end{tabular}
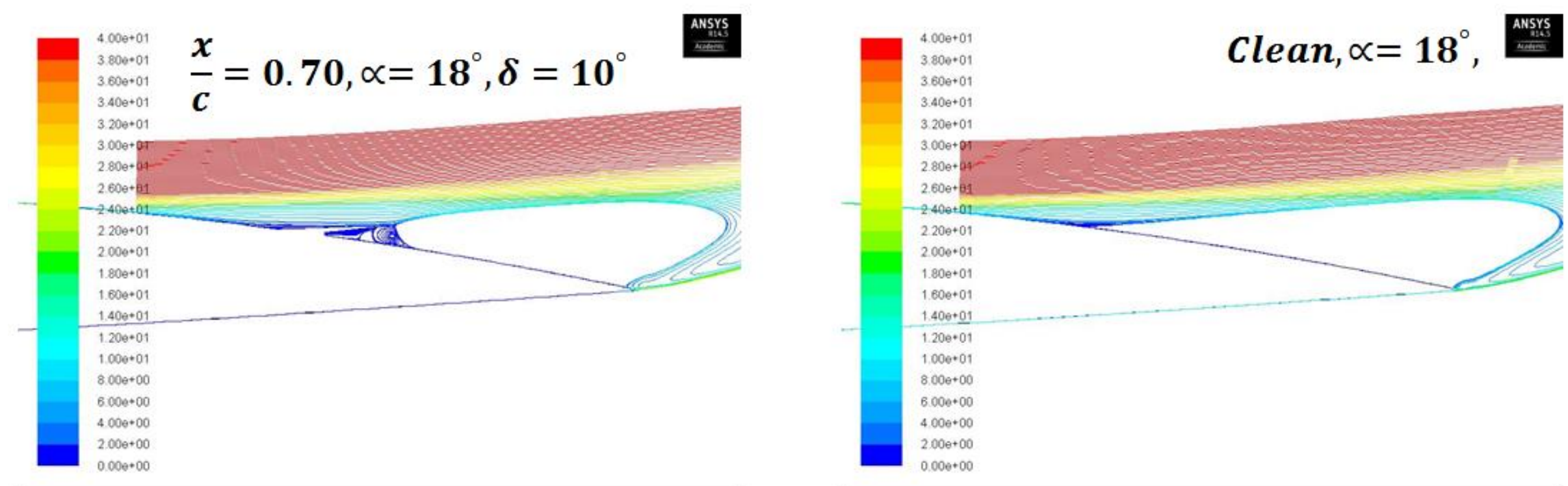


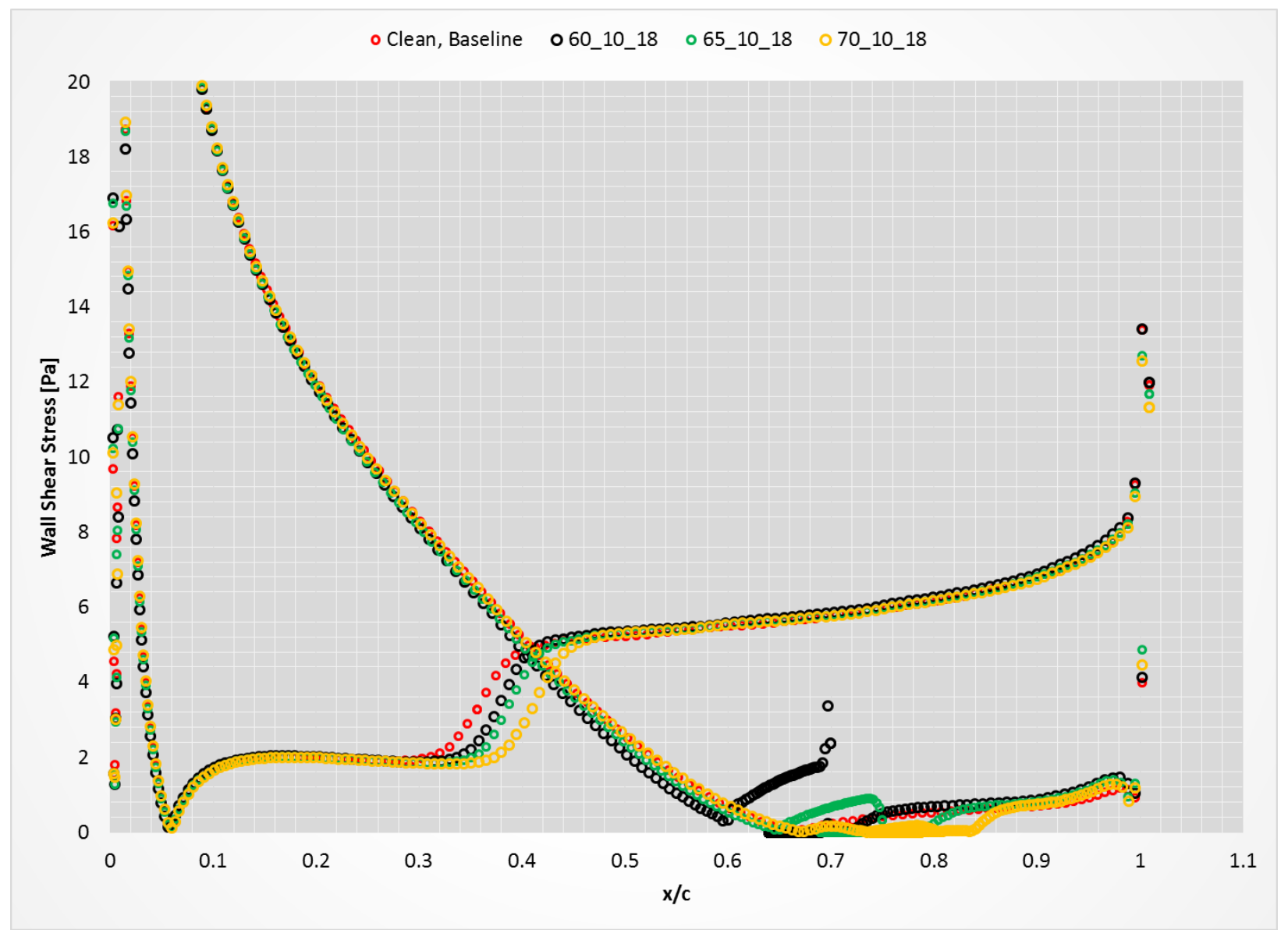

Figure 96: Wall Shear Stress, Alpha=18, Spoiler Deflection $=10, M=0.17, R e=3.79 E 6$, Transition SST Model 


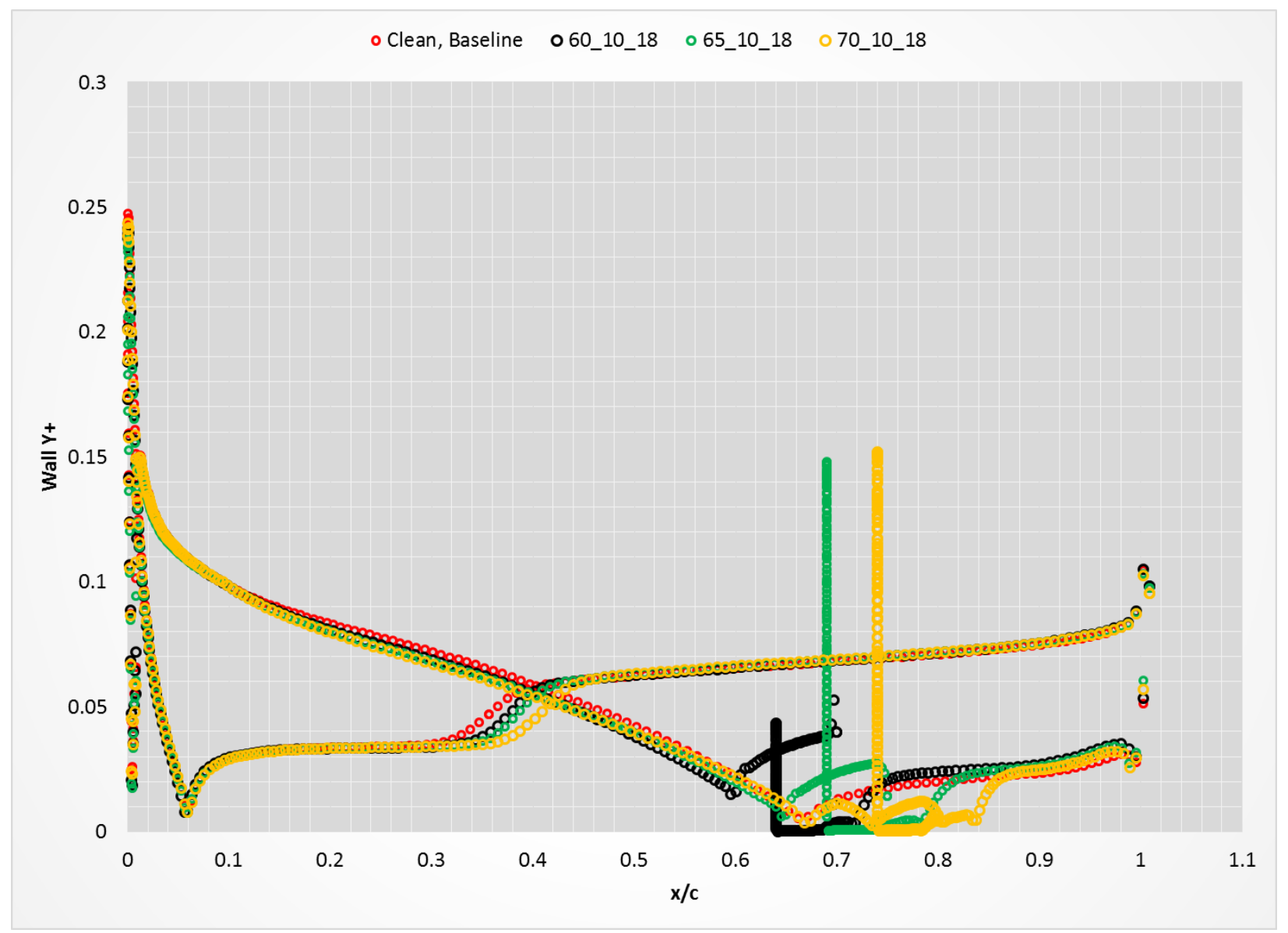

Figure 97: Wall Y+, Alpha=18, Spoiler Deflection=10, $M=0.17$, Re=3.79E6, Transition SST Model 


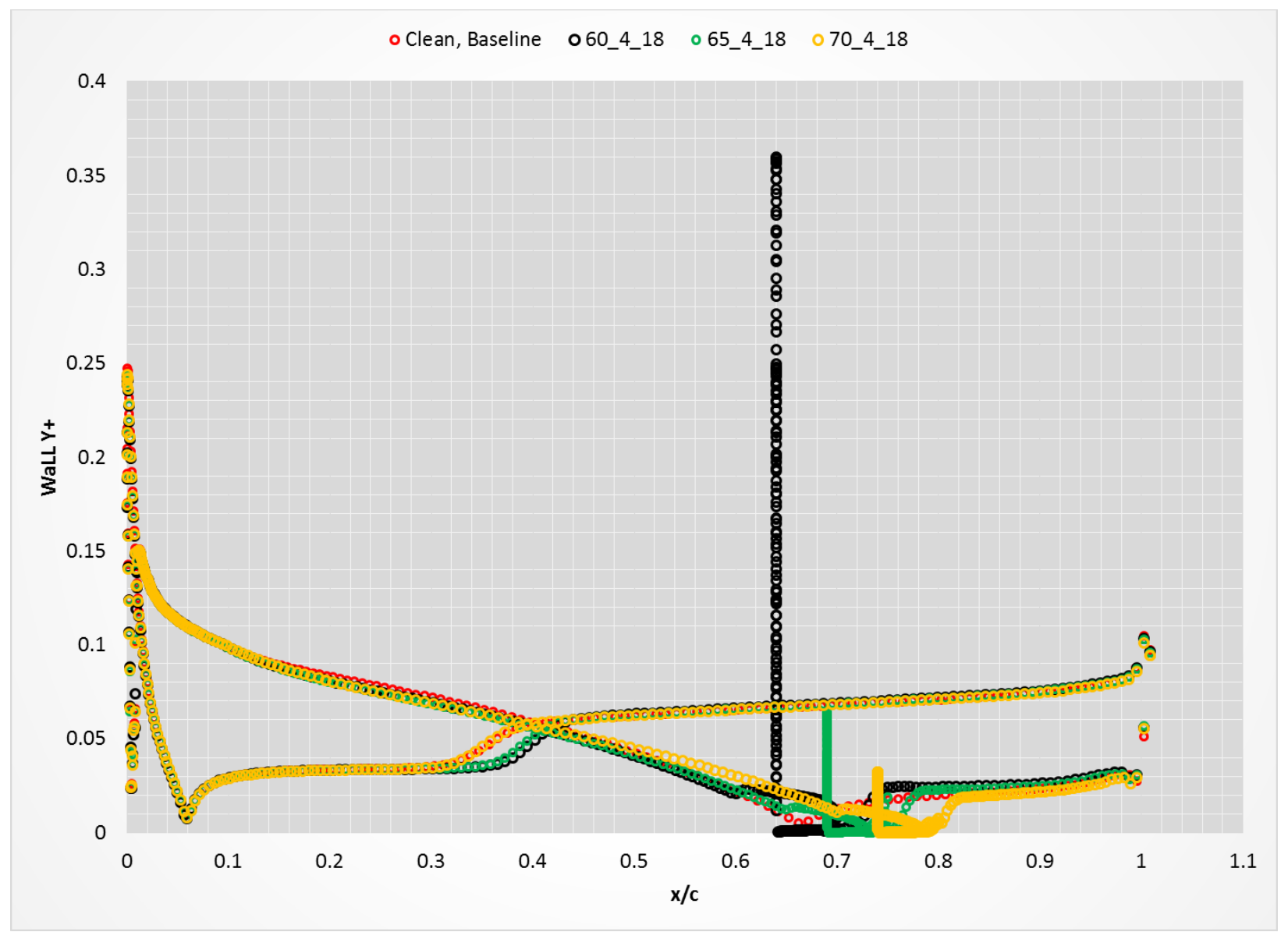

Figure 98:Wall Y+, Alpha=18, Spoiler Deflection=4, $M=0.17$, Re=3.79E6, Transition SST Model 
$\underline{\text { Alpha }=19^{\circ}, \text { Spoiler Deflection }=10^{0:}}$

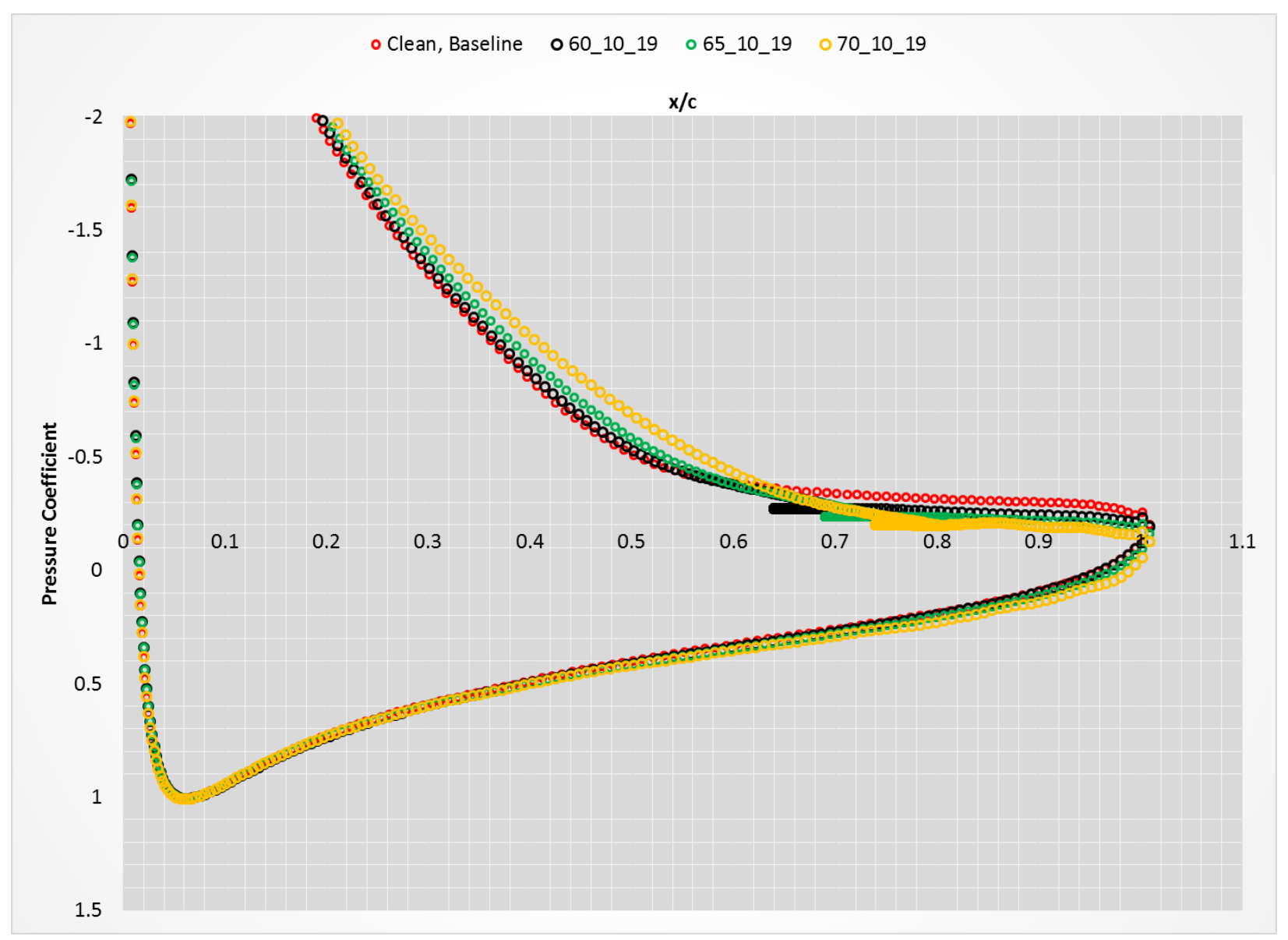

Figure 99: Pressure Coefficient, Alpha=19, Spoiler Deflection=10, $M=0.17, \operatorname{Re}=3.79 E 6$, Transition SST Model 

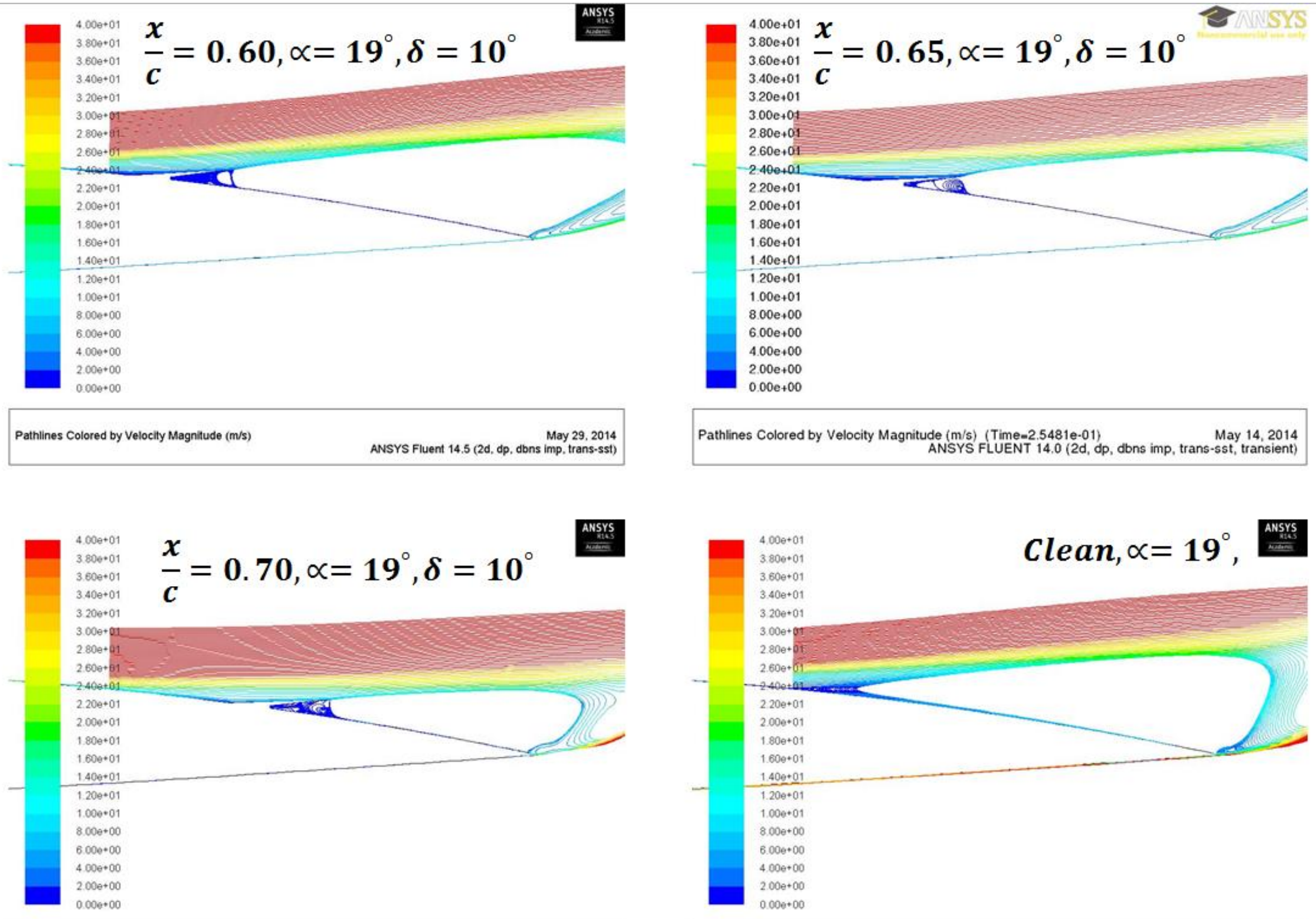


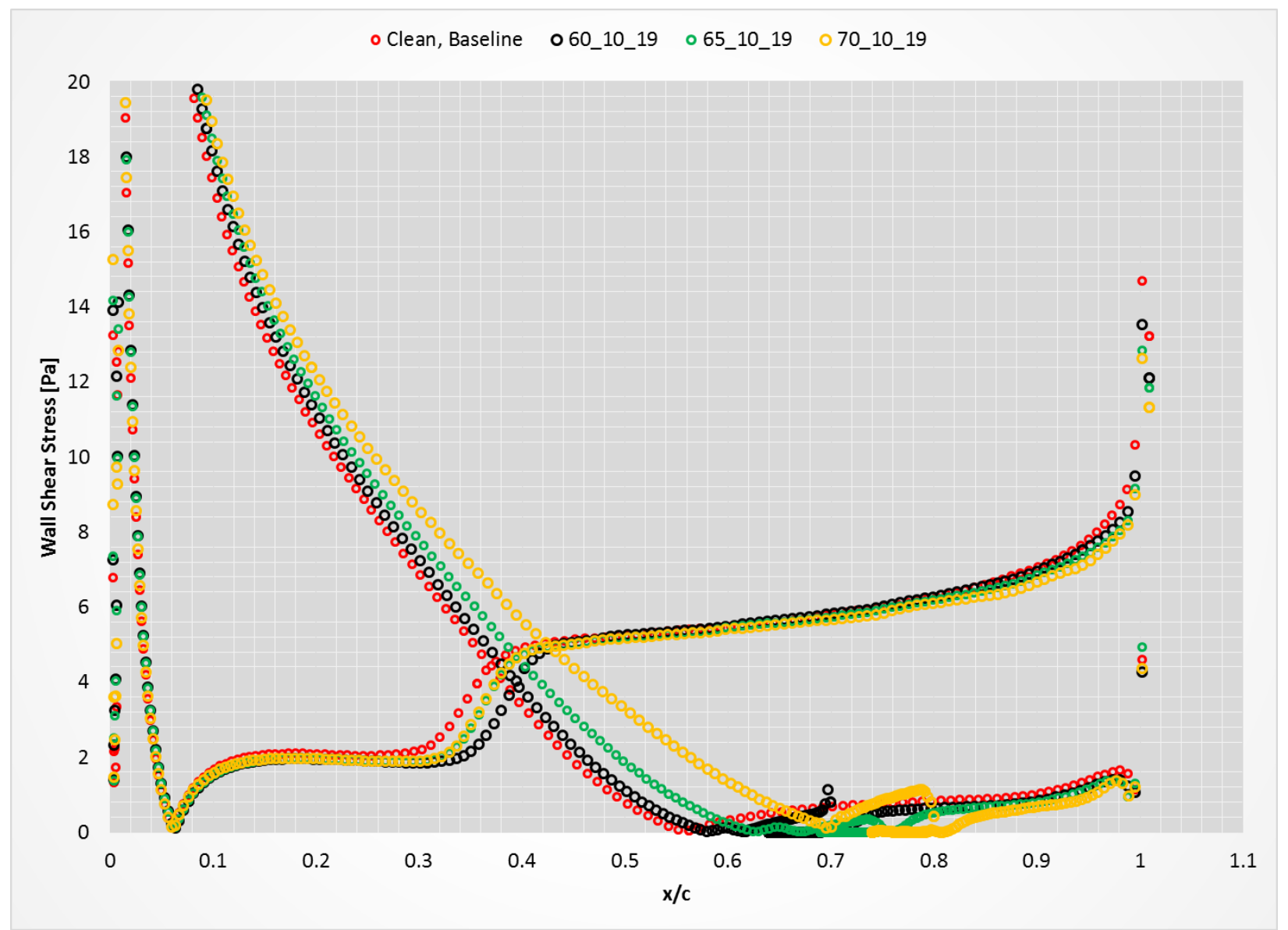

Figure 101: Wall Shear Stress, Alpha=19, Spoiler Deflection=10, $M=0.17$, Re=3.79E6, Transition SST Model 


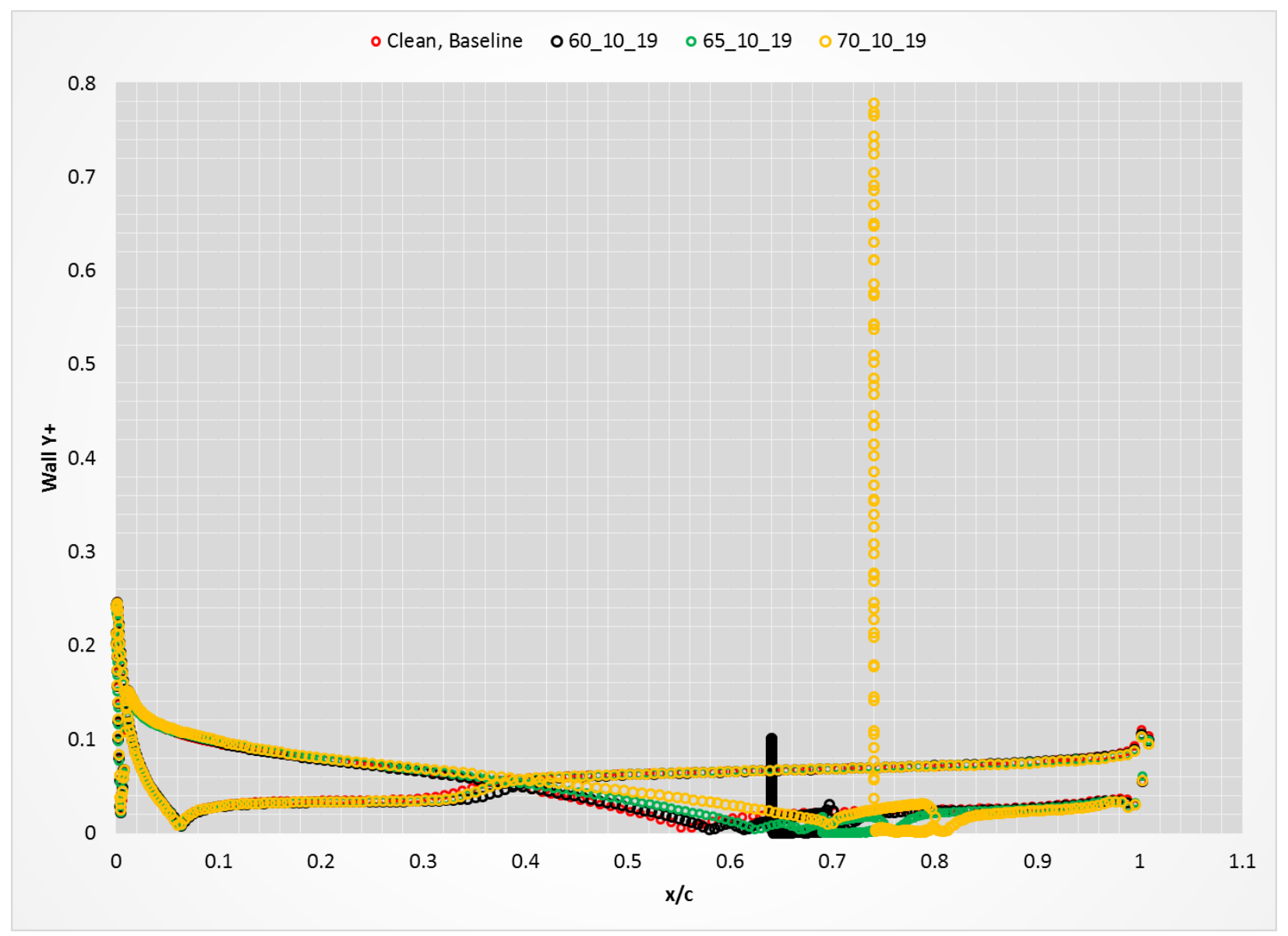

Figure 102: Wall Y+, Alpha=19, Spoiler Deflection $=10, M=0.17, R e=3.79 E 6$, Transition SST Model 


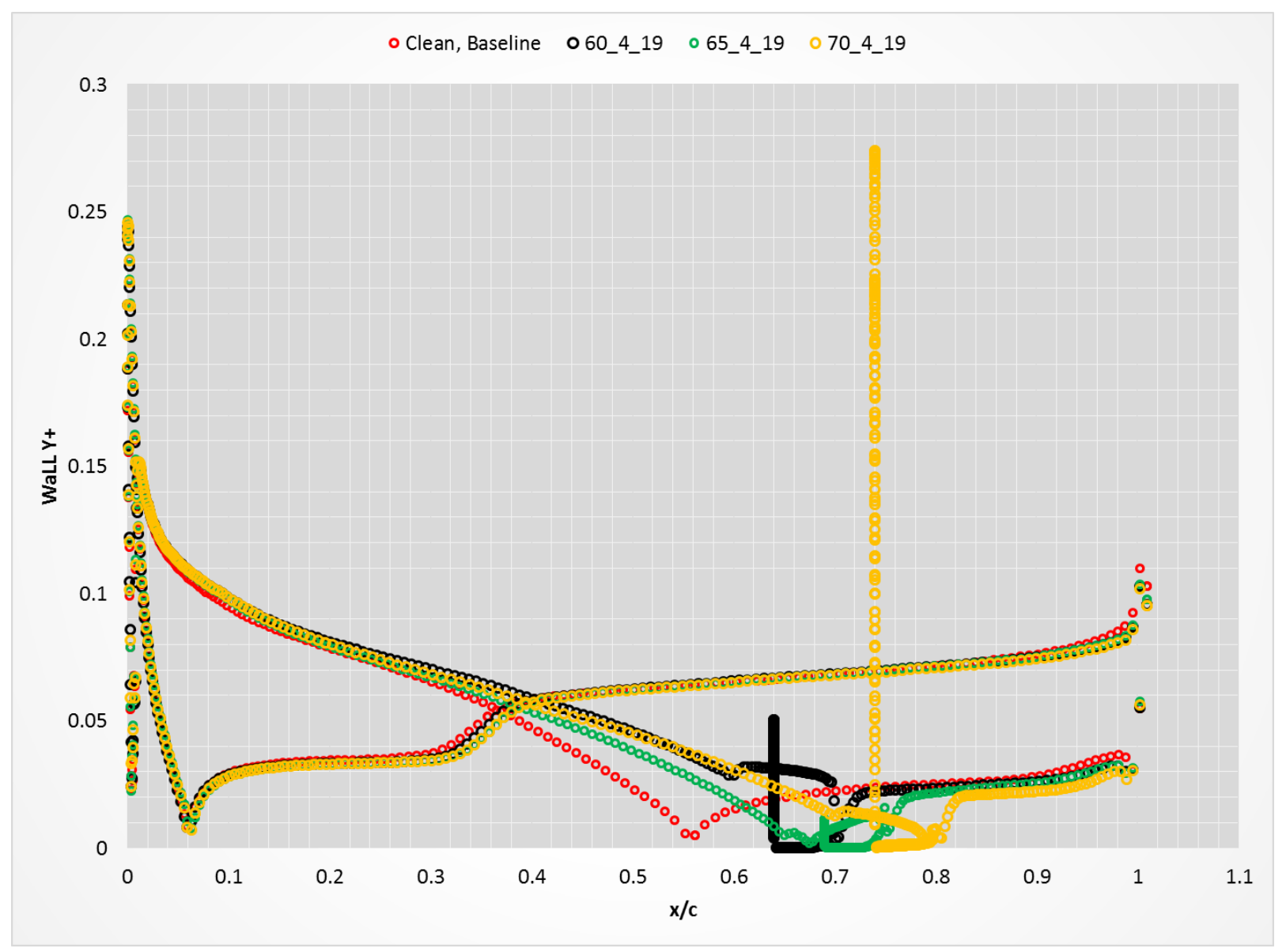

Figure 103: Wall Y+, Alpha=19, Spoiler Deflection=4, $M=0.17$, Re=3.79E6, Transition SST Model 


\section{$\underline{\text { Alpha }=20^{\circ}, \text { Spoiler Deflection }=4^{0:}}$}

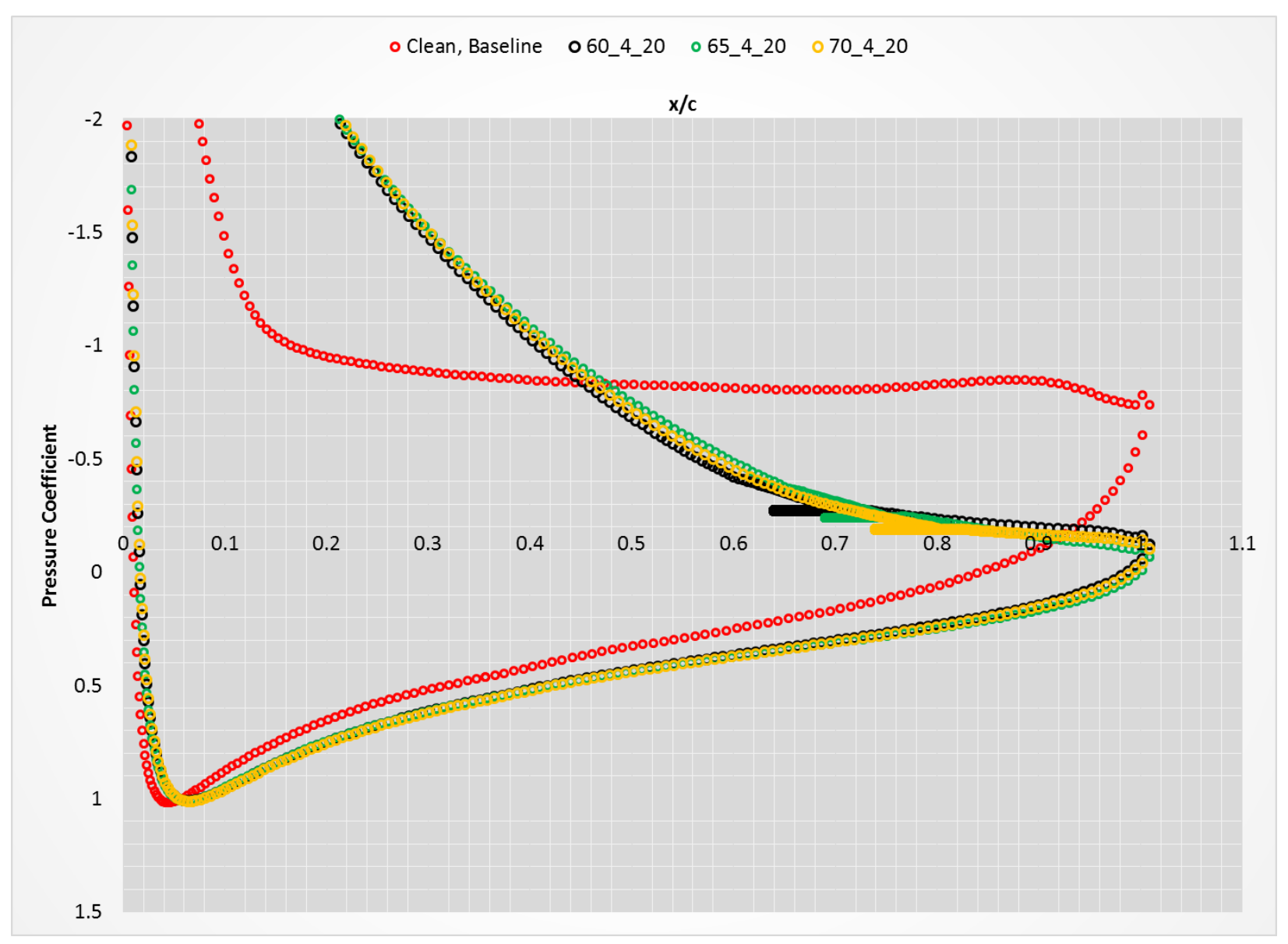

Figure 104: Pressure Coefficient, Alpha=20, Spoiler Deflection=4, M=0.17, Re=3.79E6, Transition SST Model 


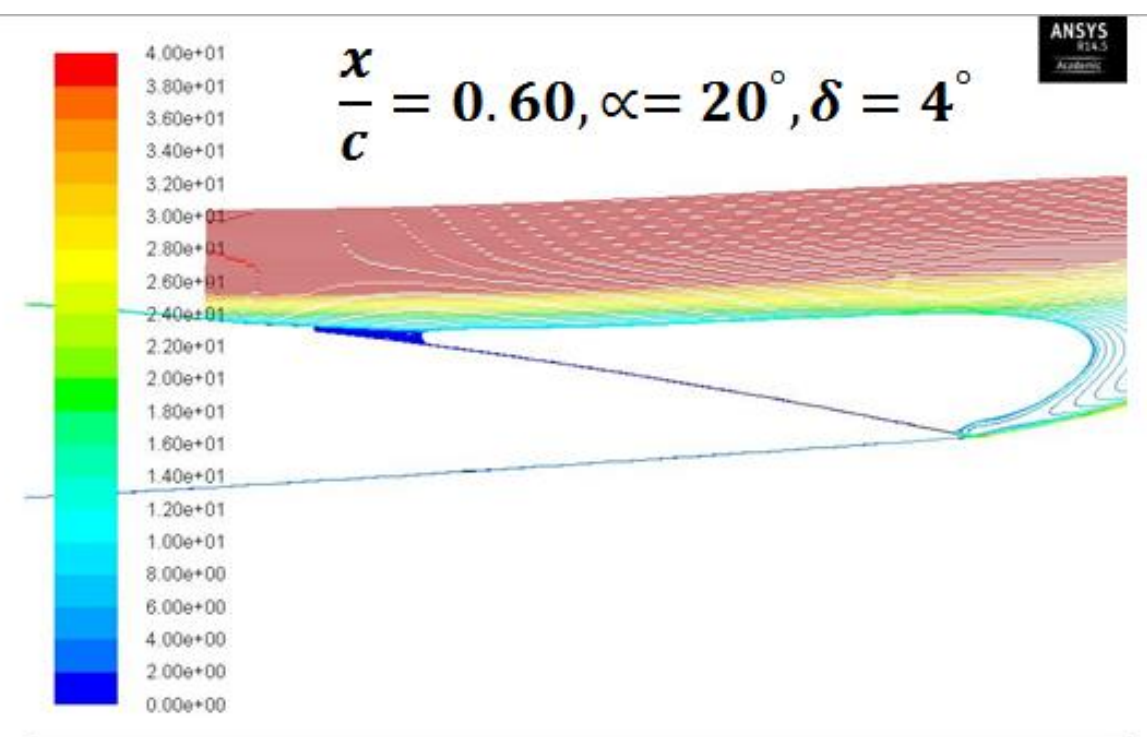

Pathlines Colored by Velocily Magnitude $(\mathrm{m} / \mathrm{s})($ Time $=8.7710 \mathrm{e}-02)$

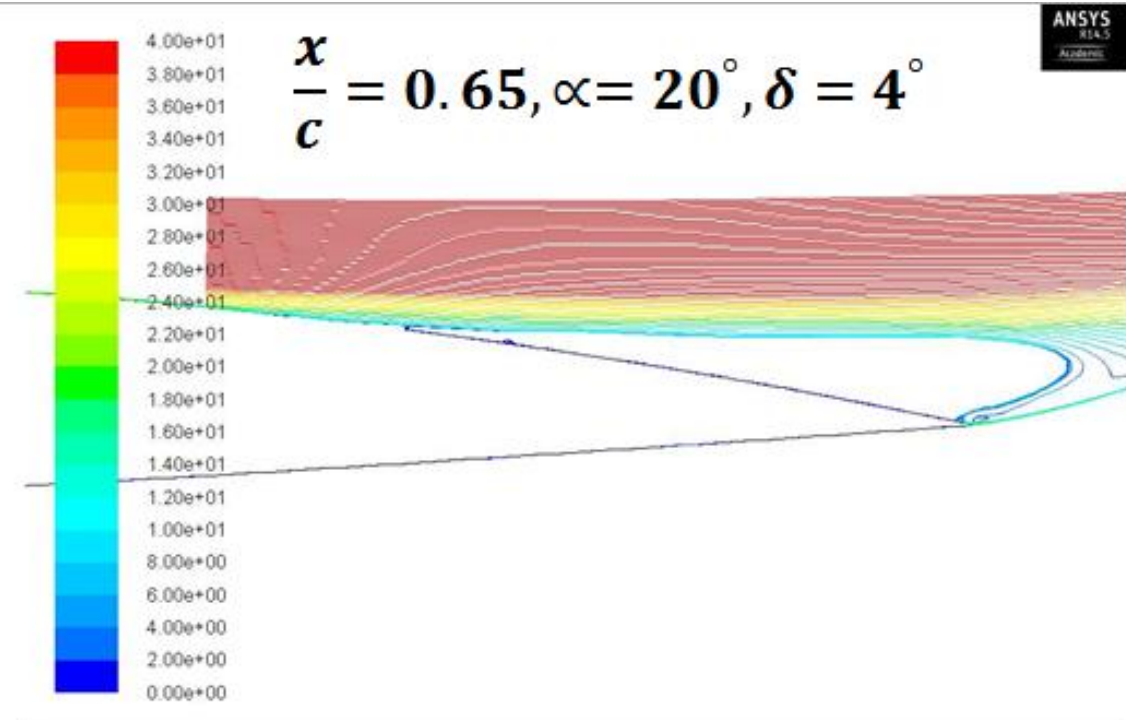

Pathlines Colored by Velocily Magnlude ( $\mathrm{m} / \mathrm{s}$ ) (Time=6.7390e- 02$)$
ANSYS Fluent 14.5 (2d, dp, dbns imp, trans-sst, transient)
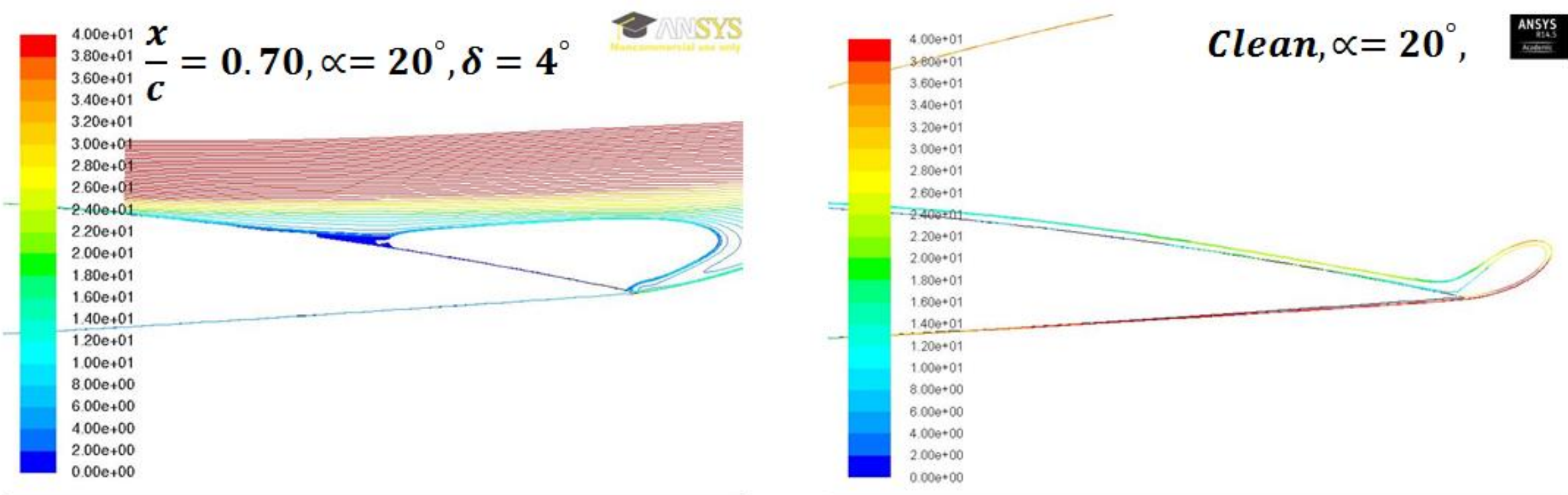

Pathlines Colored by Velocity Magnitude (m/s) (Time-9.2330e-02) May 30, 2014
ANSYS FLUENT 14.0 (2d, dp, dbns imp, trans-sst, transient)

Pathlines Colored by Velocity Magnilude $(\mathrm{m} / \mathrm{s})($ Time $=2.2680 \mathrm{e}-01) \quad J u n)^{23,2014}$ Jun 23,2014
ANSYS Fluent 14.5 (2d, dp. dbns imp, trans-sst, transient) 


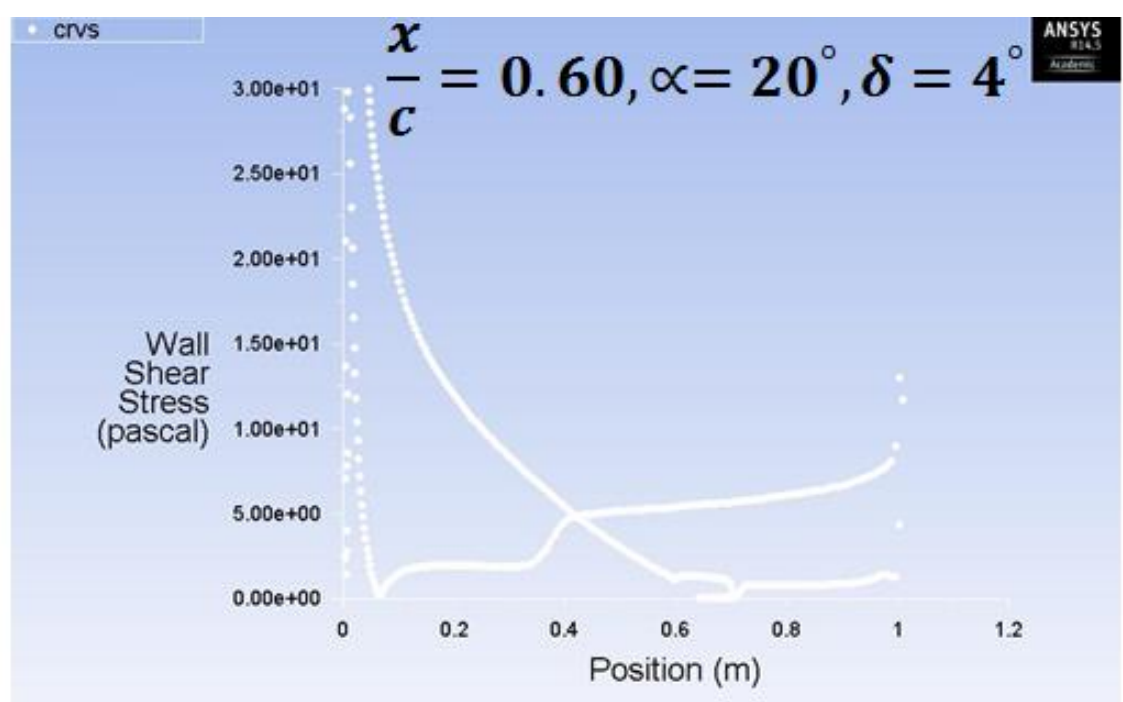

Wall Shear Stress (Time $=8.7710 \mathrm{e}-02$ )

ANSYS Fluent 14.5 (2d, dp, dbns imp, trans-sst. transient)

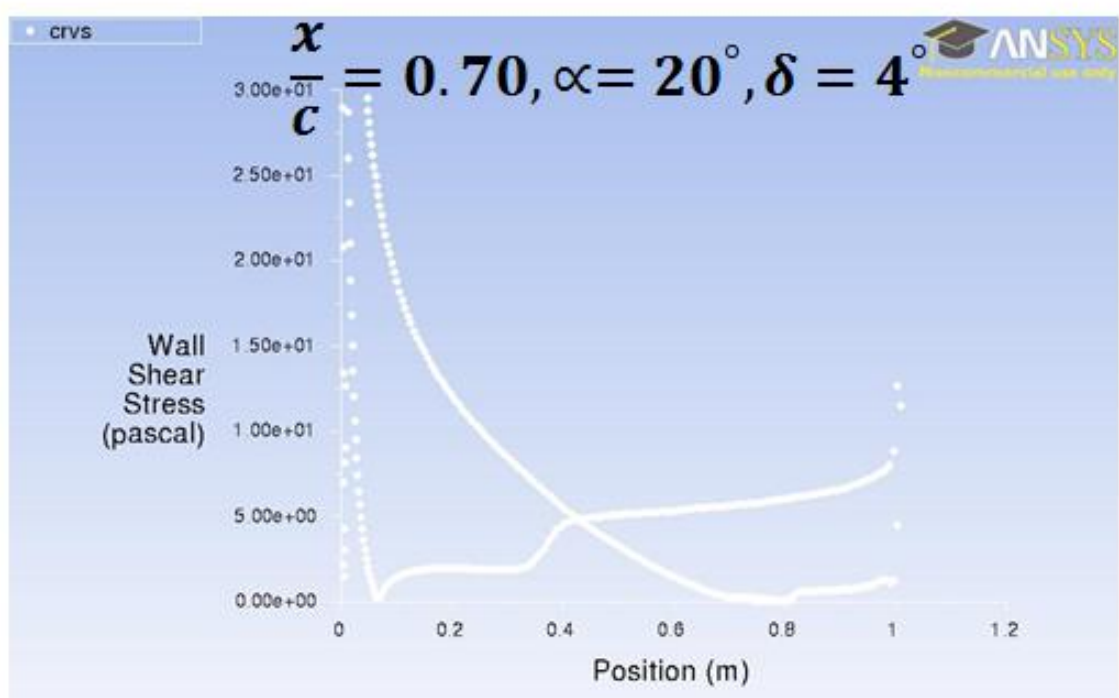

Wall Shear Stress (Time-9.2330e-02)

ANSYS FLUENT 14.0 (2d, dp, dbns imp trans-sst, 30,2014

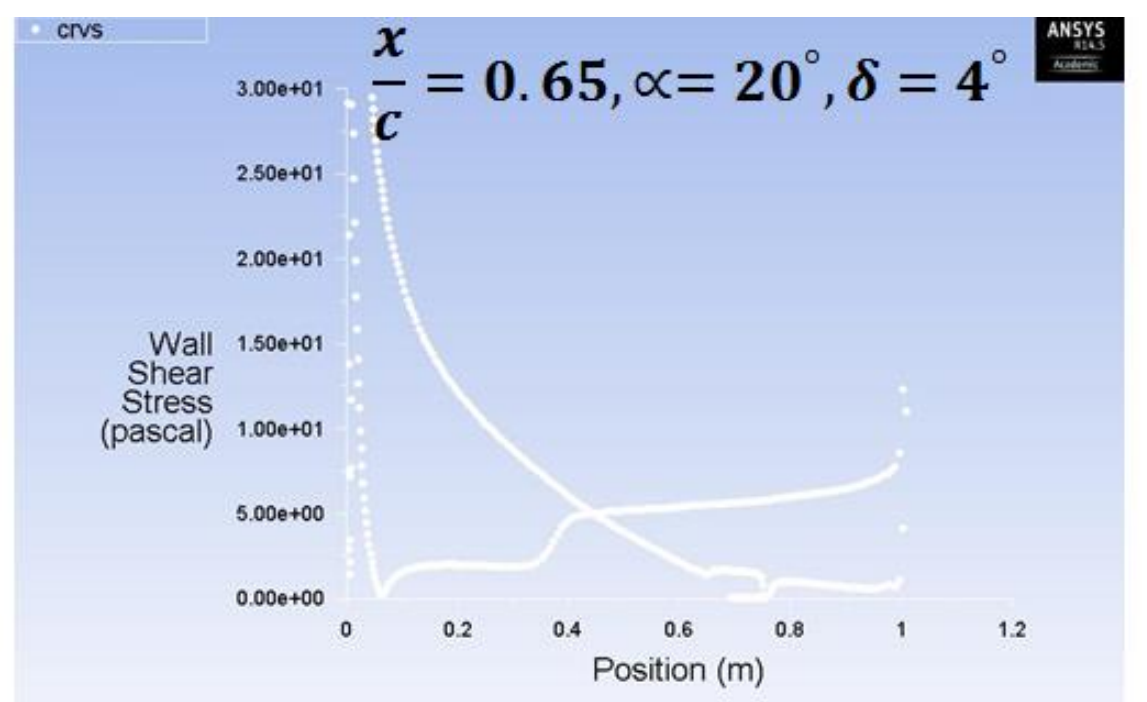

Wall Shear Stress (Time $=6.7390 \mathrm{e}-02)$ May 28, 2014
ANSYS Fluent 14.5 (2d, dp, dbns imp, trans-sst, transient)

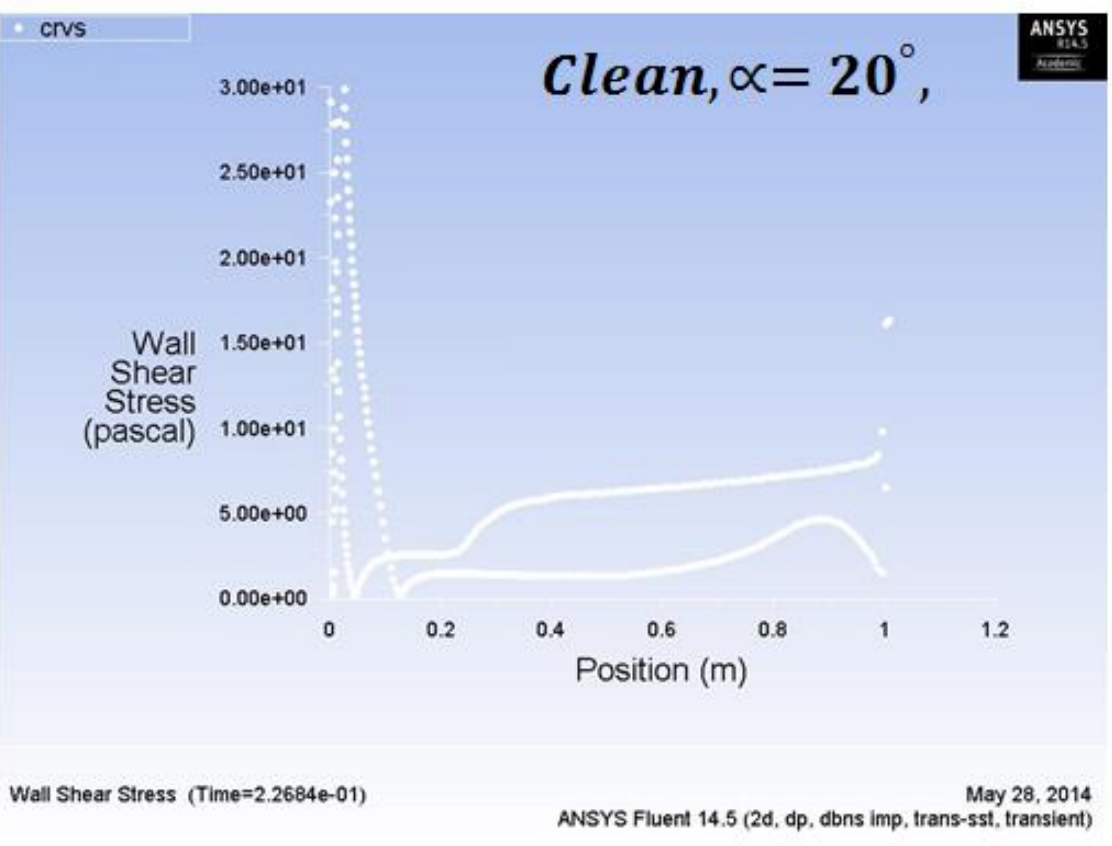




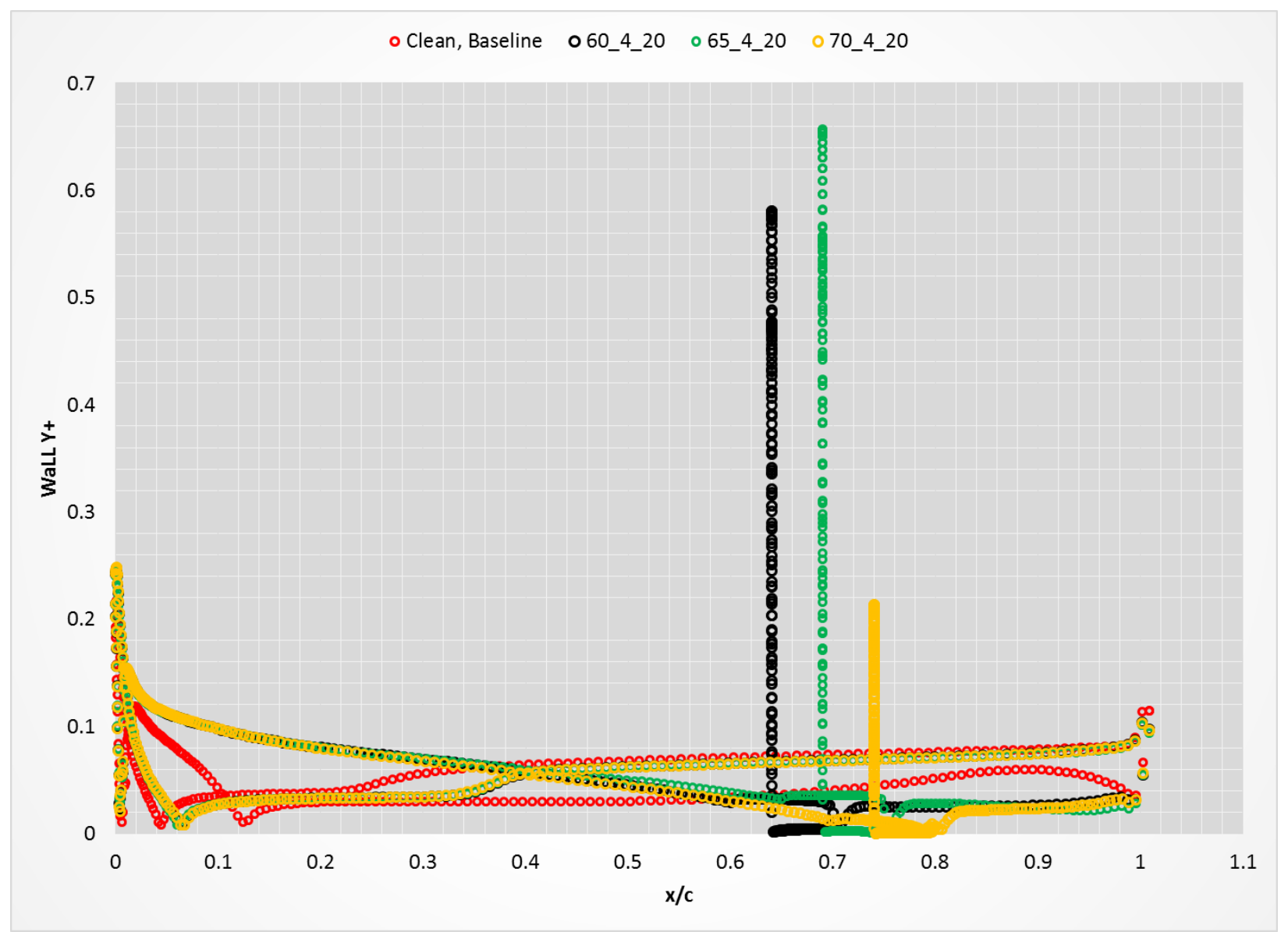

Figure 107: Wall Y+, Alpha=20, Spoiler Deflection $=4, M=0.17$, Re=3.79E6, Transition SST Model 


\section{$\underline{\text { Alpha }=21^{\circ}, \text { Spoiler Deflection }=4^{0:}}$}

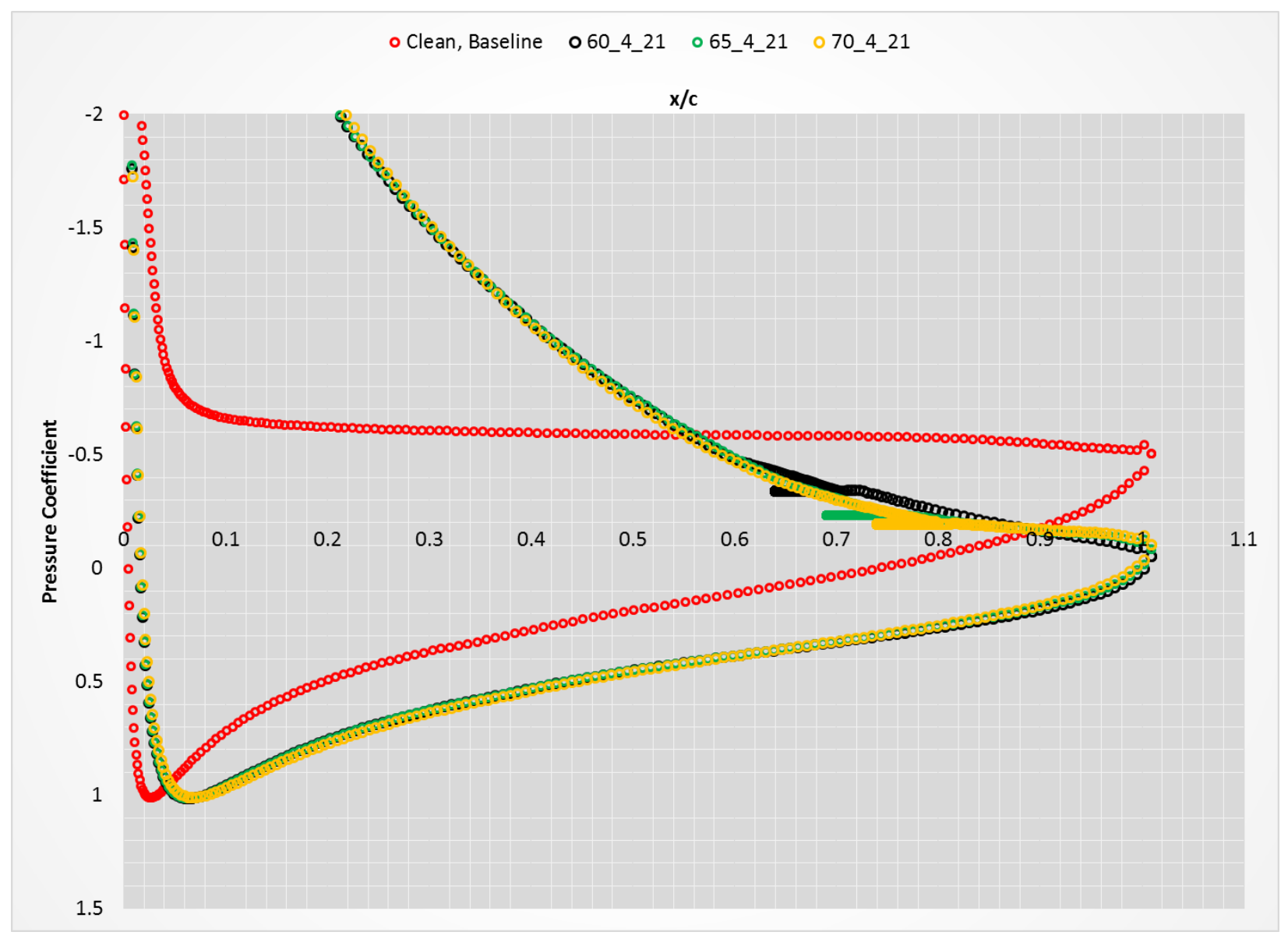

Figure 108: Pressure Coefficient, Alpha=21, Spoiler Deflection=4, $M=0.17, \operatorname{Re}=3.79 E 6$ 

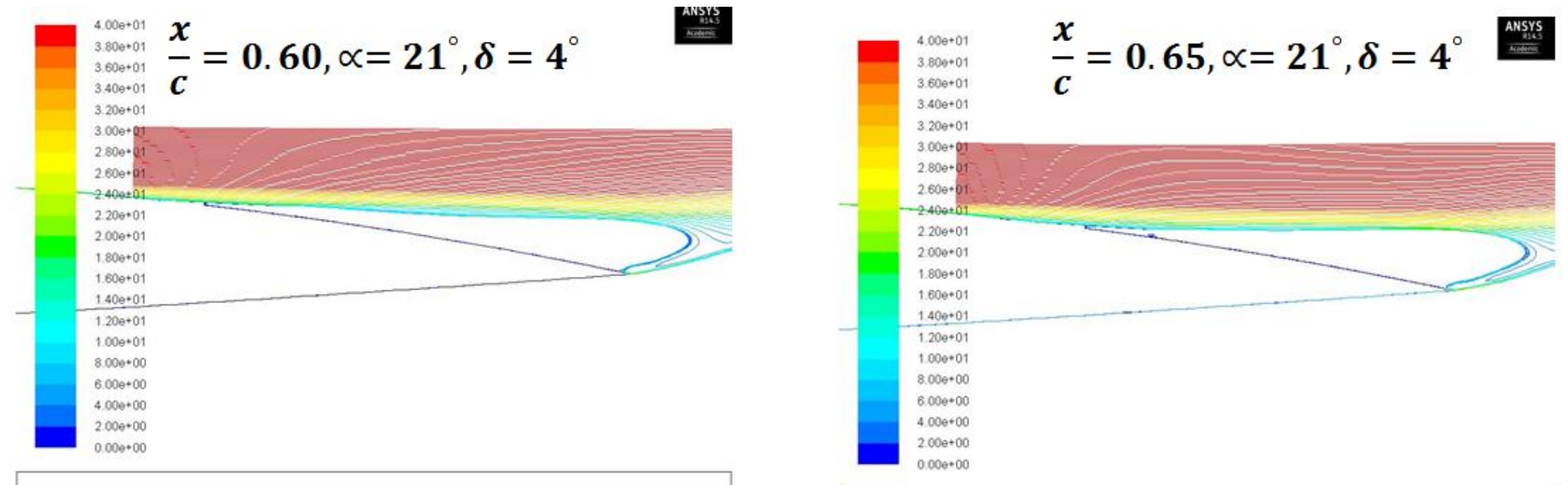

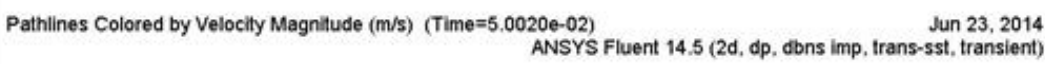

Pathlines Colored by Velocity Magnilude ( $\mathrm{m} / \mathrm{s})$ (Time=5.0000e-02) Jun 23,2014 ANSYS Fluent 14.5 (2d, dp, dbns imp, trans-sst, transient)
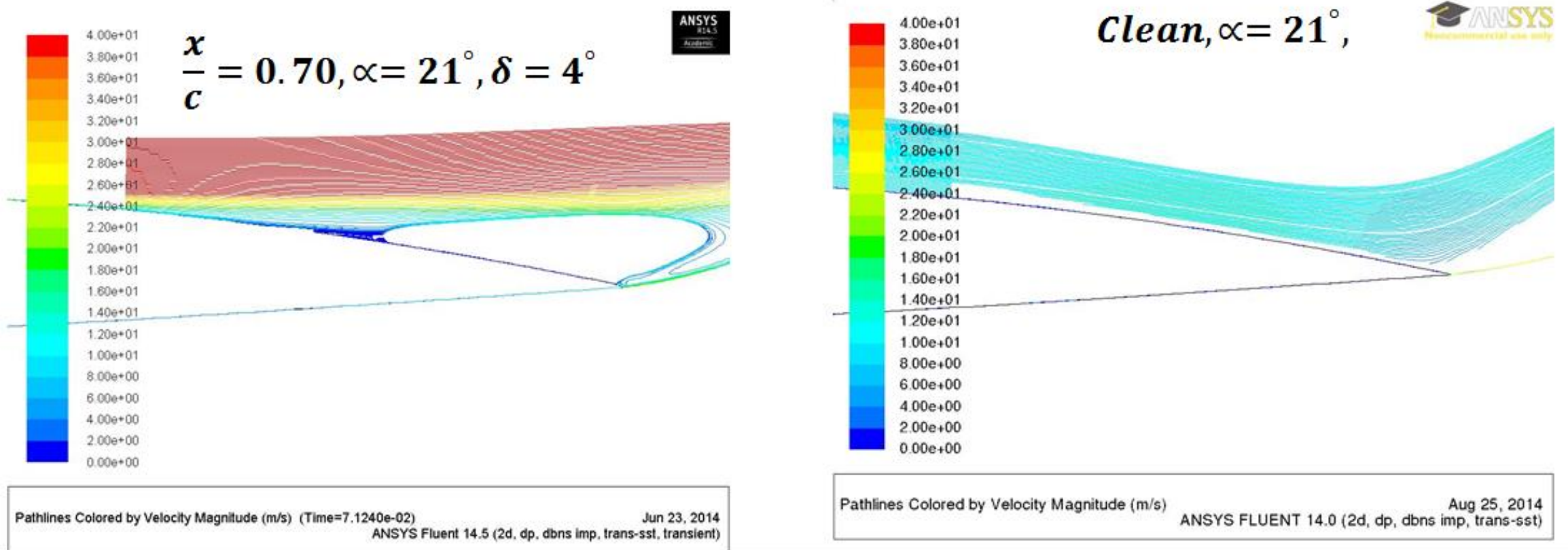


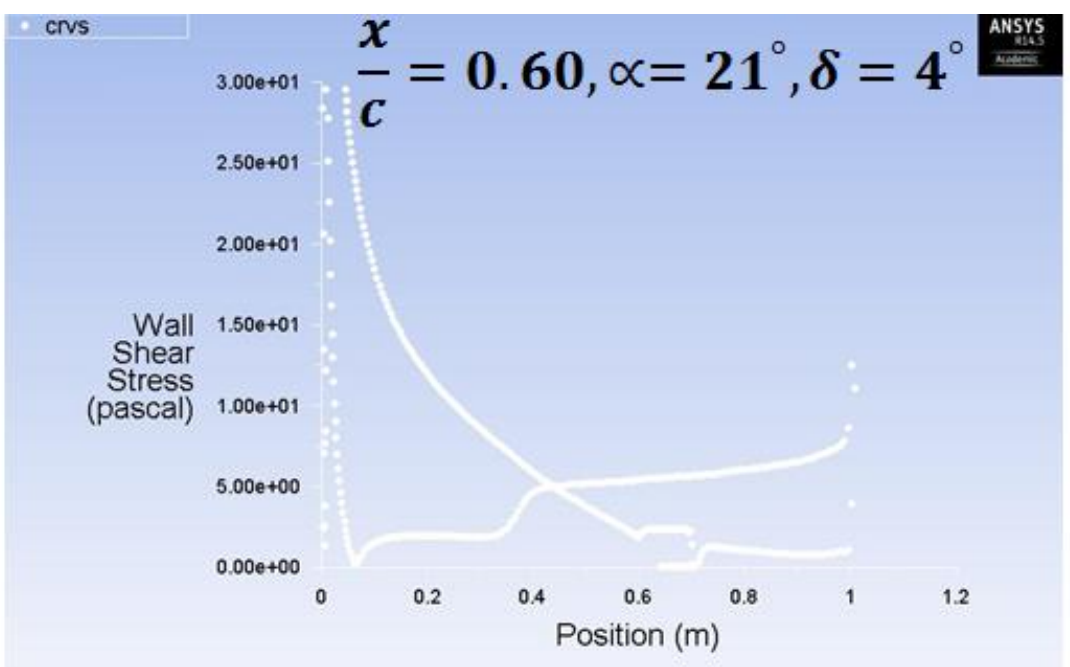

Wall Shear Stress (Time $=5.0020 \mathrm{e}-02)$

Jun 23, 2014

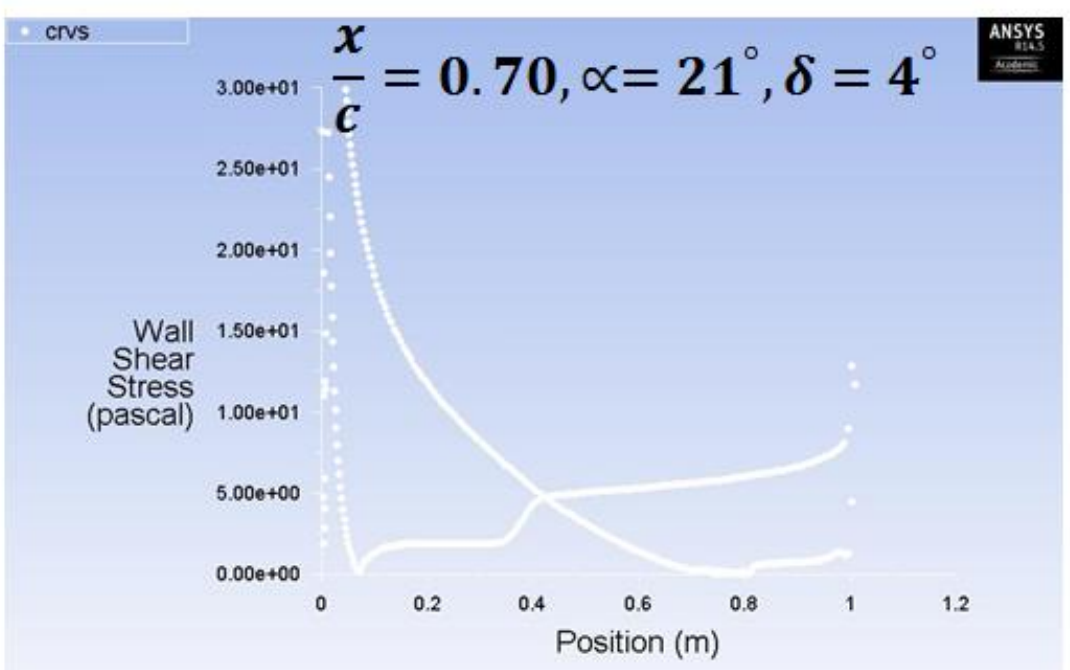

Wall Shear Stress (Time=7.1240e-02)

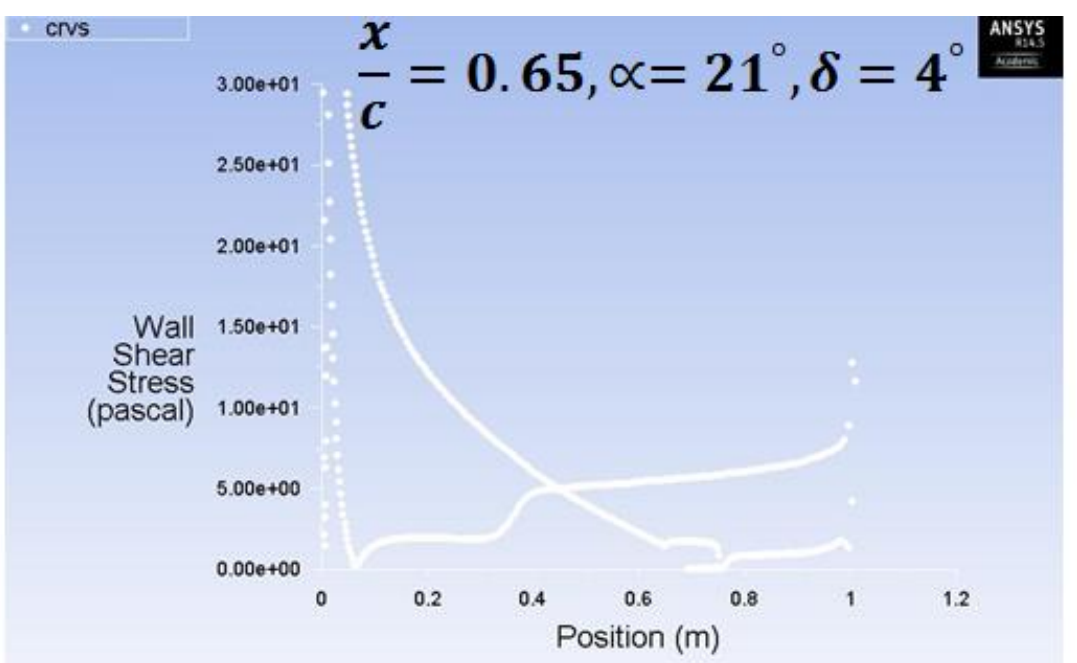

Wall Shear Stress (Time $=5.0000 \mathrm{e}-02)$

Jun 23, 2014

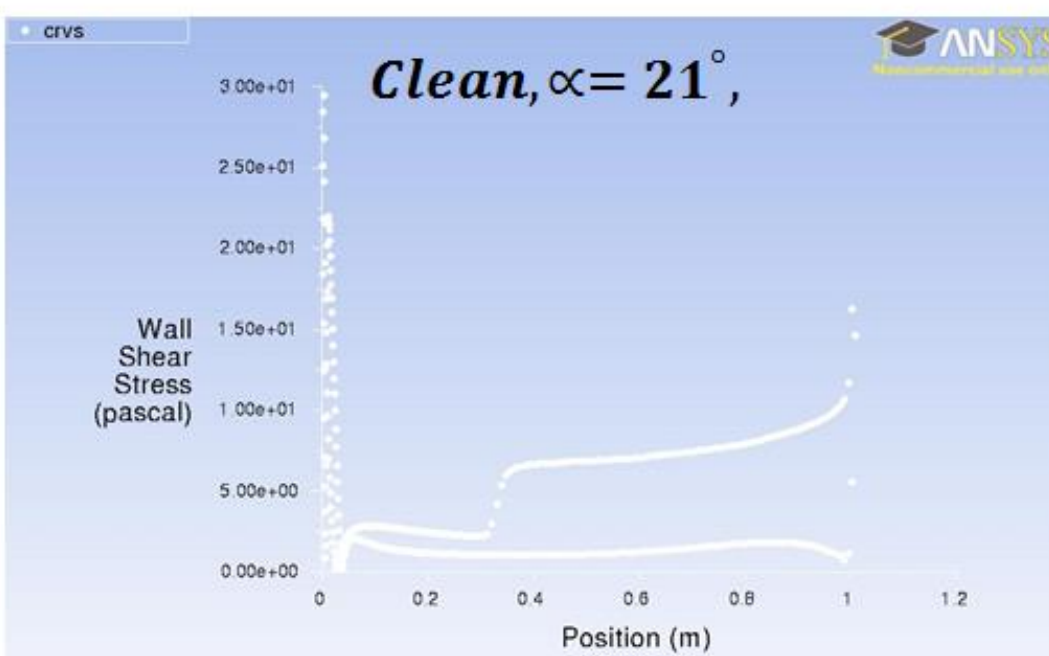

Wall Shear Stress

ANSYS FLUENT 14.0 (2d, Aug 25, 2014

Figure 110:Wall Shear Stress, Alpha=21, Spoiler Deflection $=4, M=0.17, \operatorname{Re}=3.79 E 6$ 


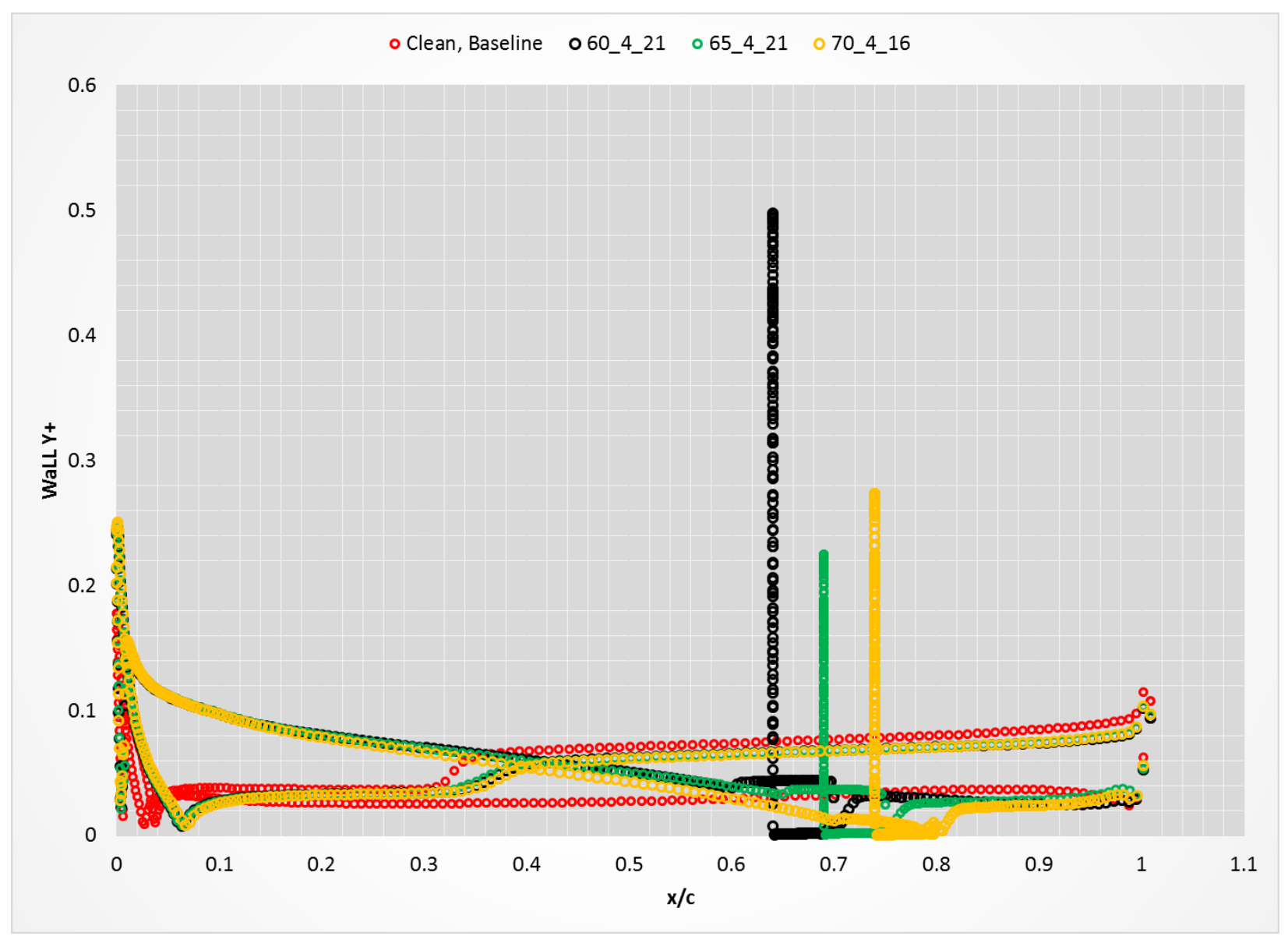

Figure 111: Wall Y+, Alpha=21, Spoiler Deflection=4, $M=0.17, R e=3.79 E 6$ 
$\underline{\text { Alpha }=23^{\circ}, \text { Spoiler Deflection }=4^{0:}}$

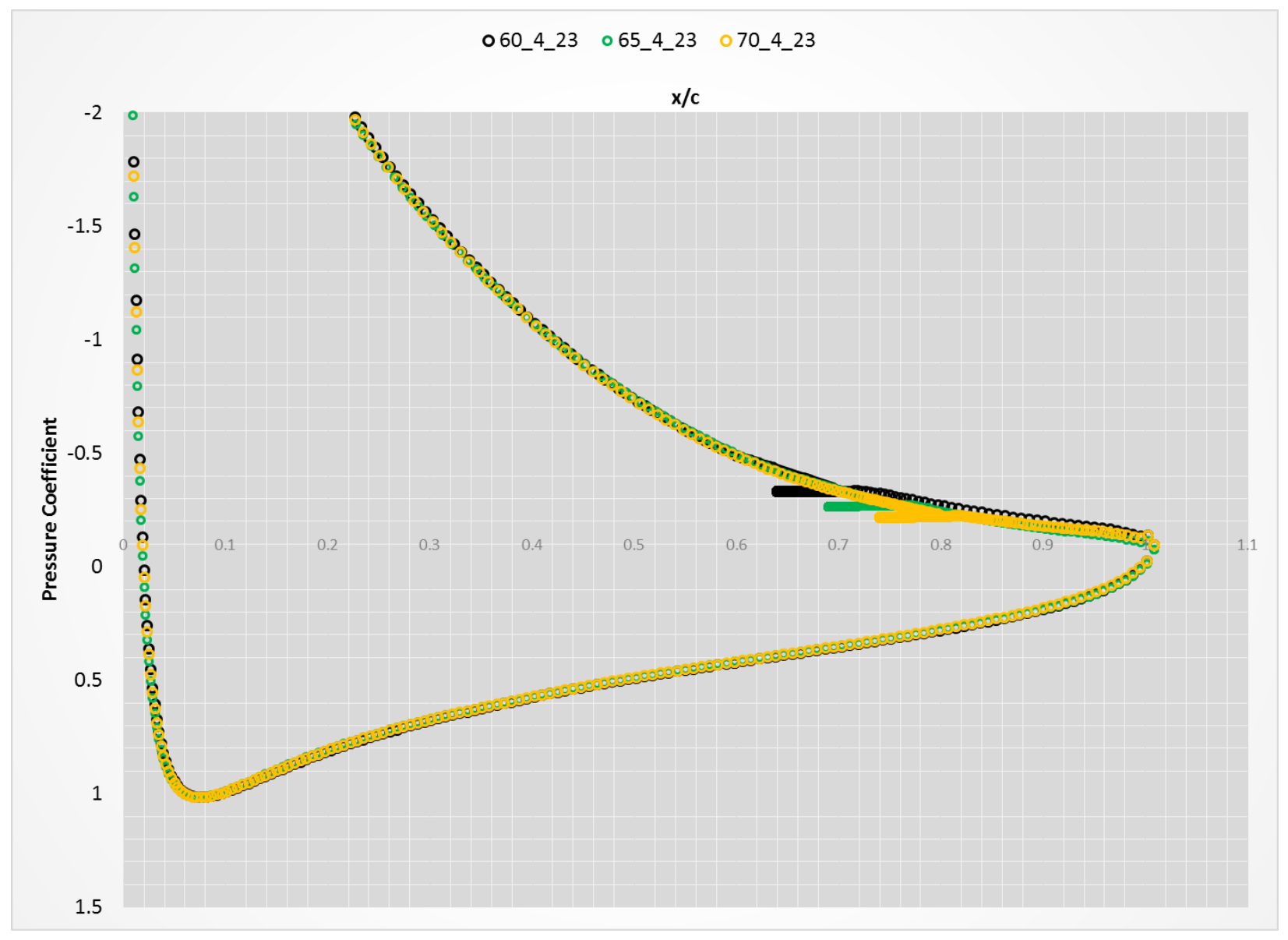

Figure 112: Pressure Coefficient, Alpha=23, Spoiler Deflection $=4, M=0.17, R e=3.79 E 6$ 


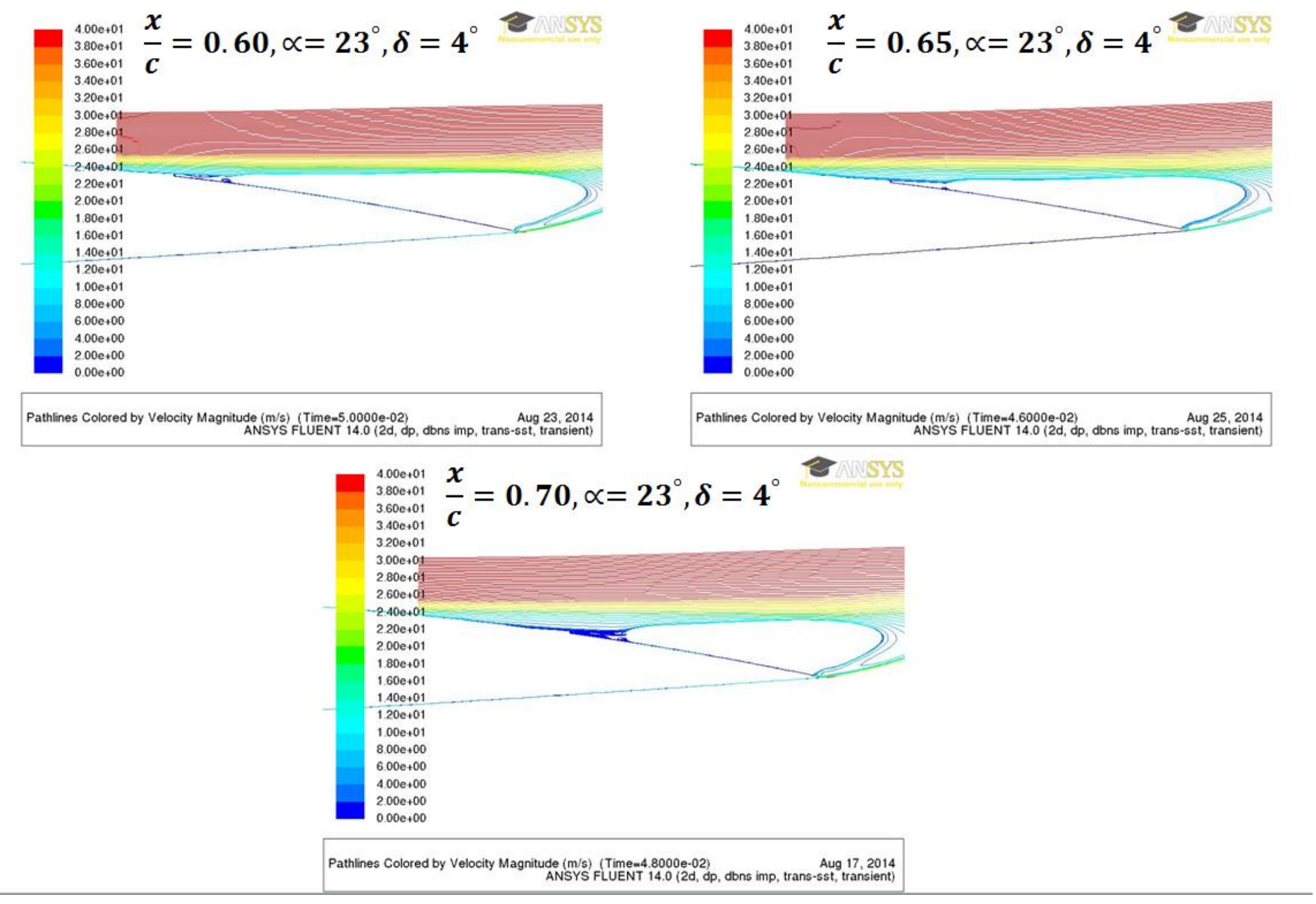

Figure 113: Velocity coloured Streamlines, Alpha $=23$, Spoiler Deflection $=4, M=0.17, R e=3.79 E 6$ 


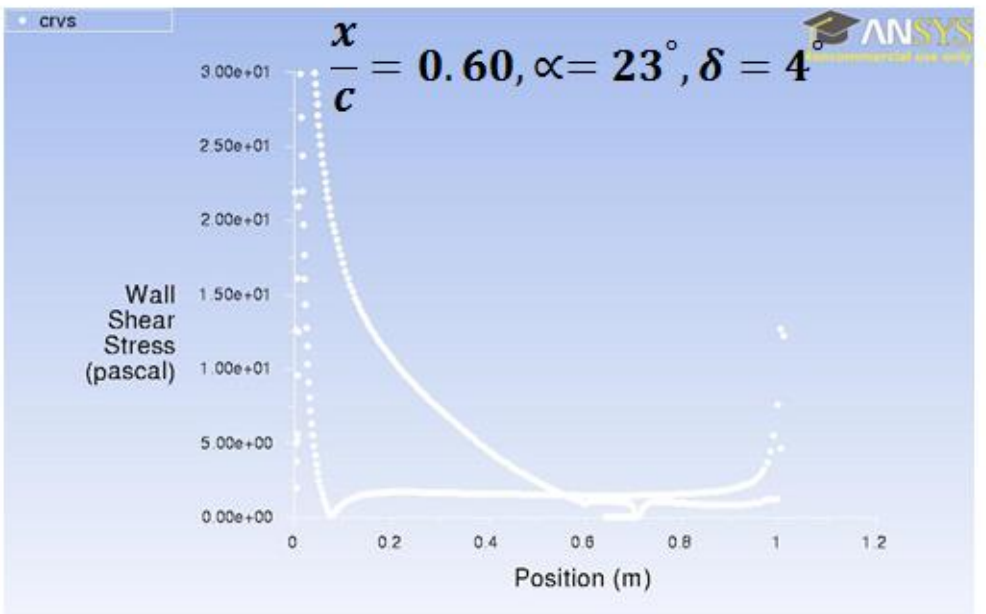

Wall Shear Stress (Time- $5.0000 \mathrm{e}-02$ ) ANSYS FLUENT 14.0 (2d, dp, dbns imp, trans-sst, transient)

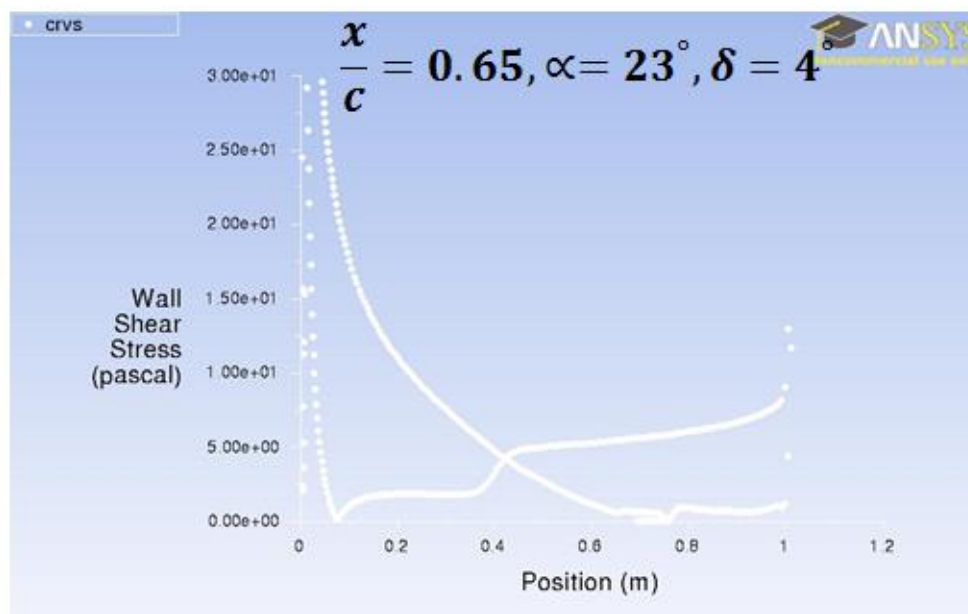

Wall Shear Stress (Time-4.6000e-02)
ANSYS FLUENT 14.0 (2d, dp, dbns imp, trans-sst, transient)

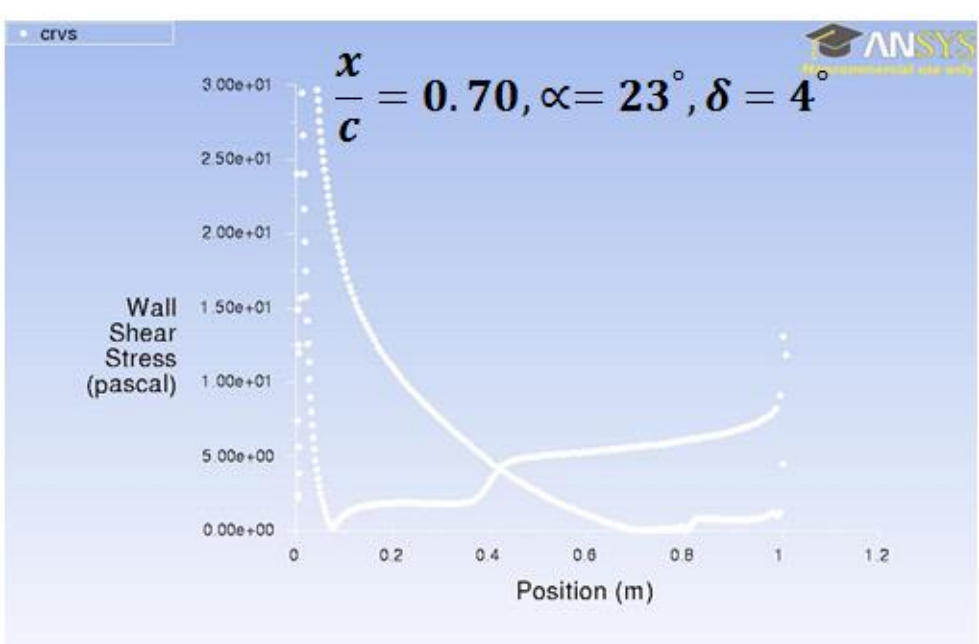

Wall Shear Stress (Time $=4.8000$ e-02) ANSYS FLUENT 14.0 (2d, dp, dbns imp, trans- Aug 17, 2014

Figure 114: Spoiler Study, Wall Shear Stress, Alpha $=23$, Spoiler Deflection $=4, M=0.17, R e=3.79 E 6$, Transition SS 


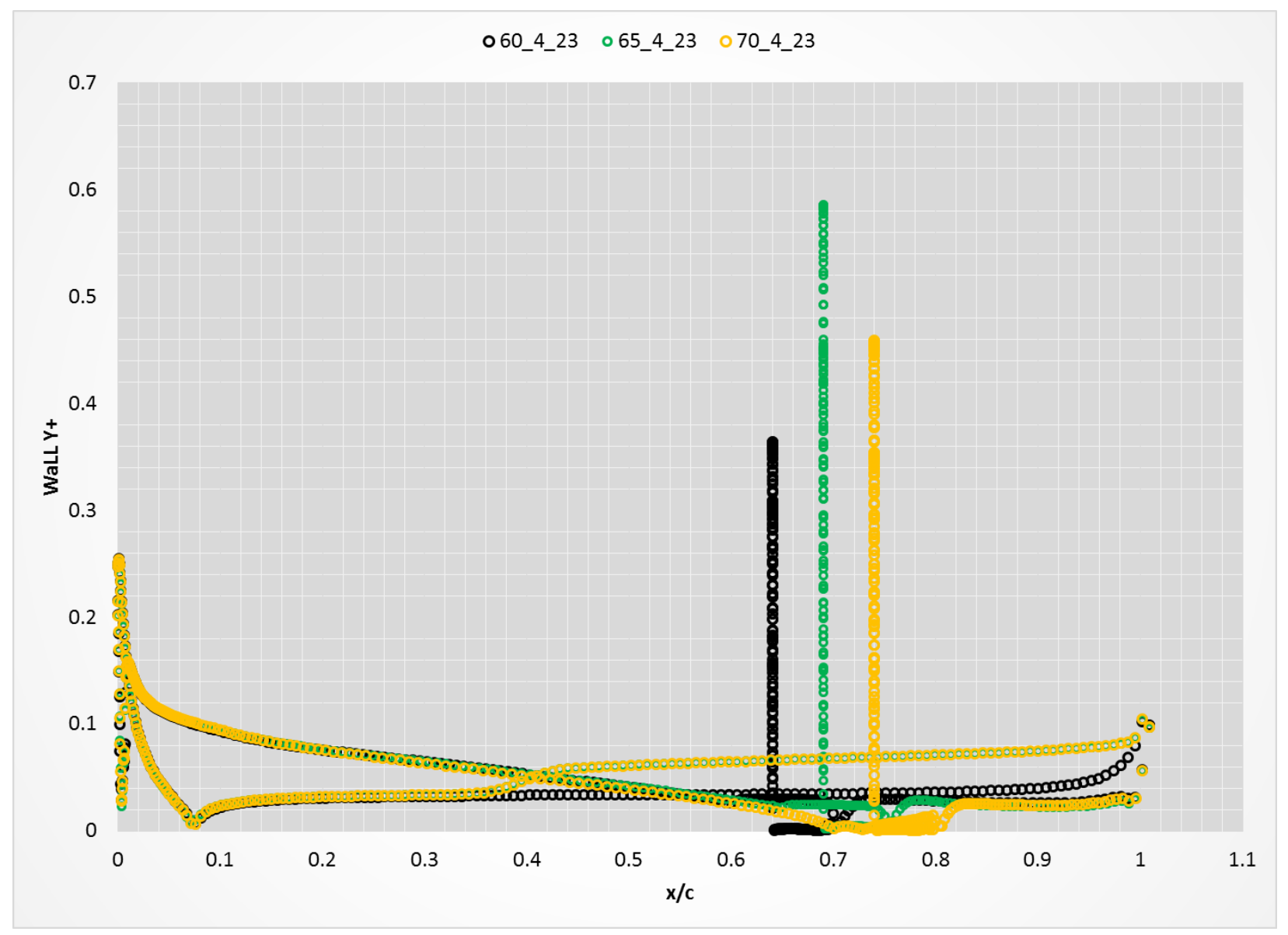

Figure 115: Wall $Y+$, Alpha=23, Spoiler Deflection $=4, M=0.17, R e=3.79 E 6$ 


\section{Appendix D: Shear Stress and Navier-Stokes Equation}

Shear stress in the physical sense is recognized as a response to deformation. Commonly explained through popular literary books, shear stress is a force per unit area and considering one side of a 6-faced cubic volume unit as an example, deformation can occur due to forces acting both perpendicular to the plane as well as within the plane under consideration. Therefore, Shear stress can be decomposed into two forces: a surface normal and two tangential acting in the plane of a two dimensional surface.

In 1849, G.G. Stokes provided an 'acceptable' relation between the shear to pressure forces present in the normal direction. Without delving into the derivation, the result forms the commonly known Navier-Stokes equations of fluid motion:

$$
\begin{gathered}
\rho \frac{D \vec{u}}{D t}=f_{x}-\frac{\partial p}{\partial x}+\frac{\partial}{\partial x}\left[\mu\left(2 \frac{\partial u}{\partial x}-\frac{2}{3} \operatorname{div} \vec{v}\right)\right]+\frac{\partial}{\partial y}\left[\mu\left(\frac{\partial u}{\partial y}+\frac{\partial v}{\partial x}\right)\right]+\frac{\partial}{\partial z}\left[\mu\left(\frac{\partial w}{\partial x}+\frac{\partial u}{\partial z}\right)\right] \\
\rho \frac{D \vec{v}}{D t}=f_{y}-\frac{\partial p}{\partial y}+\frac{\partial}{\partial y}\left[\mu\left(2 \frac{\partial v}{\partial y}-\frac{2}{3} \operatorname{div} \vec{v}\right)\right]+\frac{\partial}{\partial z}\left[\mu\left(\frac{\partial v}{\partial x}+\frac{\partial w}{\partial y}\right)\right]+\frac{\partial}{\partial x}\left[\mu\left(\frac{\partial u}{\partial y}+\frac{\partial v}{\partial x}\right)\right] \\
\rho \frac{D \vec{w}}{D t}=f_{z}-\frac{\partial p}{\partial z}+\frac{\partial}{\partial z}\left[\mu\left(2 \frac{\partial w}{\partial z}-\frac{2}{3} \operatorname{div} \vec{v}\right)\right]+\frac{\partial}{\partial x}\left[\mu\left(\frac{\partial w}{\partial x}+\frac{\partial u}{\partial z}\right)\right]+\frac{\partial}{\partial y}\left[\mu\left(\frac{\partial v}{\partial z}+\frac{\partial w}{\partial y}\right)\right] \\
\operatorname{div} \vec{v}=\left(\frac{\partial \vec{u}}{\partial \vec{x}}+\frac{\partial \vec{v}}{\partial \vec{y}}+\frac{\partial \vec{w}}{\partial \vec{z}}\right)
\end{gathered}
$$

In turbulence modelling, the stress terms in the Navier-Stokes equation is the focus for numerical approximation. One very well-known hypothesis that attempts to quantify the shear stress terms in was devised by Boussinesq in 1877. In a nutshell, Boussinesq proposed an equation that best explains the mixing of the strong velocity gradients present in turbulent flow (termed "turbulent eddies"). Boussinesq suggested that there is a factor, which was termed "turbulent viscosity" that is coupled with the velocity gradient in the boundary layer. 


$$
\text { Boussinesq Equation }=-v_{T} \frac{\partial U}{\partial y}
$$

Models were then developed to attempt to approximate the "turbulent viscosity term". In literature, the theory is based on two key determinates to effectively approximate 'turbulent viscosity'; velocity and mixing length scales. Models that were formulated based on this "Eddy Viscosity" hypothesis differed in the overall formulation of either the velocity and/or mixing length scale equations.

For example, the k-epsilon model developed by Jones and Launder (1971), which is one of the most popular two equation turbulence models currently utilized to aerodynamics flows. The model approximates turbulent viscosity as a function of mixing length scale, as well as kinetic energy and density as a product of a constant:

$$
v_{T}=c \rho k^{1 / 2} l
$$

Kinetic energy from Equation [19] is isolated for through solving the turbulent kinetic energy equation:

$$
\rho \frac{D k}{D t}=\frac{\partial}{\partial y}\left(\frac{v_{T}}{\sigma_{K}} \frac{\partial k}{\partial y}\right)+v_{T}\left(\frac{\partial u}{\partial y}\right)^{2}-\rho \varepsilon
$$

In which the dissipation energy term, $\varepsilon$ is solved using the following relation:

$$
\rho \frac{D \varepsilon}{D t}=\frac{\partial}{\partial y}\left(\frac{v_{T}}{\sigma_{\varepsilon}} \frac{\partial \varepsilon}{\partial y}\right)+c_{1} \frac{\varepsilon}{k} v_{T}\left(\frac{\partial u}{\partial y}\right)^{2}-c_{2} \frac{\rho \varepsilon^{2}}{k}
$$

Therefore, a relation for mixing length scale is then created via both the kinetic and dissipative energy relations and according to Jones and Launder provides reasonable predictions in the high Reynolds Number flow regime. [20] 